Simon Daniel HÜGelmeyer

MULTI-DIMENSIONAL RADIATIVE TRANSFER IN CIRCUMSTELLAR DISKS

Dissertation

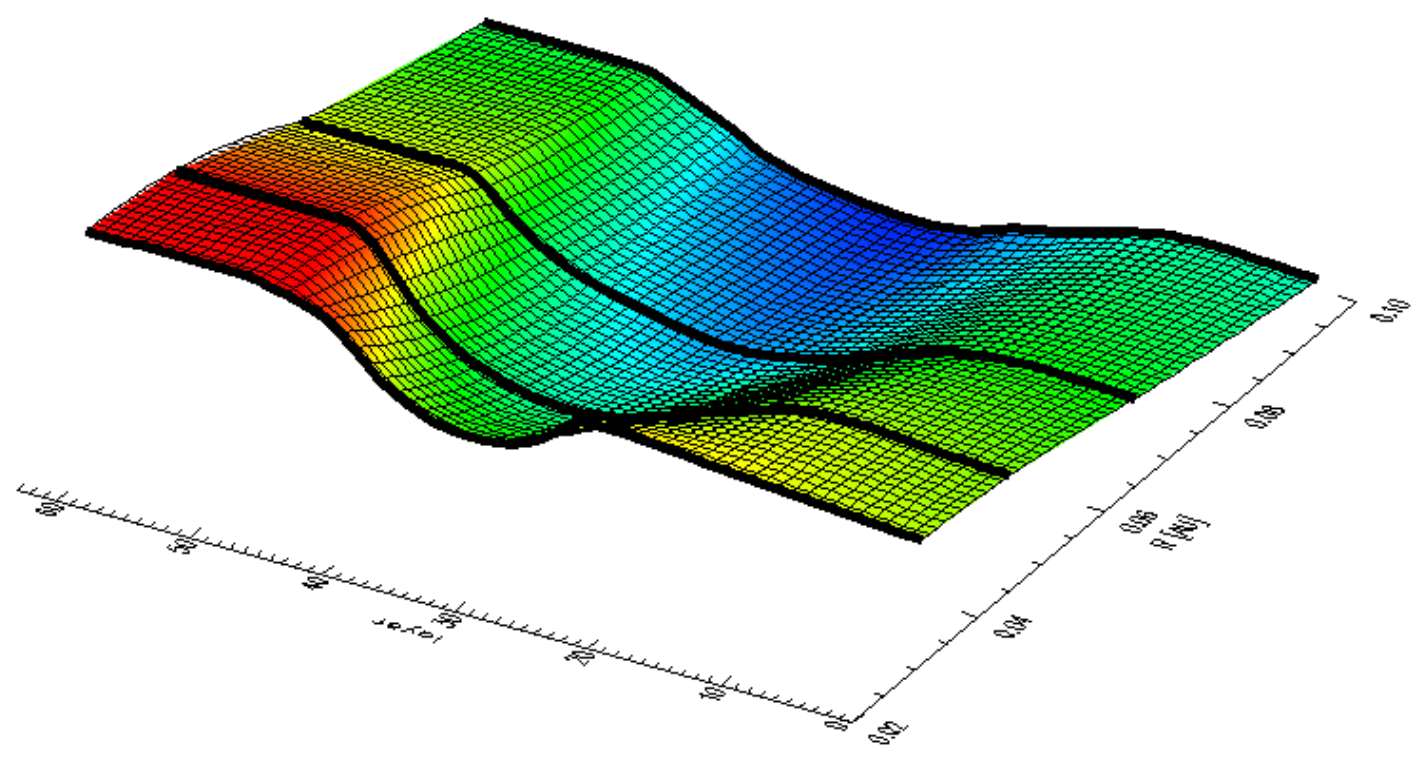





\title{
Multi-dimensional radiative transfer in circumstellar disks
}

\author{
Dissertation \\ zur Erlangung des Doktorgrades \\ der Mathematisch-Naturwissenschaftlichen Fakultäten \\ der Georg-August-Universität zu Göttingen
}

vorgelegt von

Simon Daniel Hügelmeyer

aus Osnabrück

Göttingen, 18. November 2009 
D7

Referent:

Prof. Dr. Stefan Dreizler

Koreferent:

Prof. Dr. Peter Hauschildt

Tag der mündlichen Prüfung: 17.12.2009 


\section{Abstract}

Accretion processes are common phenomena in the universe. Accretion disks can be found around various types of objects such as very young stars (e.g. T Tauri and Herbig Ae/Be stars), evolved binaries (cataclysmic variables), or massive central parts of galaxies (AGN). The cool gas and dust disks around young stellar objects, the so-called protoplanetary disks which are believed to be the birthplaces of planets, have moved to the center of attention in astrophysical research ever since the discovery of the first planets around stars other than the sun almost 15 years ago. Typically, the disk is emitting the major fraction of light from the star-disk system at wavelengths larger than the near-infrared region. Modern observing facilities provide high-quality data of IR emission from disks, e.g. spectra of high resolution, which allow to investigate the gaseous structure of the disks. Consequently, a detailed modelling of these complex systems is necessary to understand the environment of the early stages of planet formation. The goal of this thesis was to develop a program that allows to calculate the vertical structure of the inner luminous region of protoplanetary disks in detail in order to compare the emergent synthetic spectra with high-resolution observations. Furthermore, the efforts of disk modelling are extended to a full 3D treatment of the radiative transfer problem which will allow to investigate asymmetries in disks and radial radiation transport through the disk.

Early attempts to model the radiation field of accretion disks were based on blackbody spectra or stellar atmosphere models of the effective temperature of the disks. In this approach the irradiation of the central star, the height-dependent gravity in the disk, and the viscously dissipated energy are neglected and hence the models were not appropriate. Later models either accounted for the vertical structure of the disk in detail but treated the radiative transfer only approximately or accounted for dust opacities only and assumed structure properties which were not self-consistently calculated. Newer models allow to obtain full numerical solutions for the structure and the detailed radiation field for either very hot disks around white dwarfs in cataclysmic variable systems or for dust in protoplanetary disks. In this work the model efforts for protoplanetary disks are extended to a detailed vertical radiative transfer and structure calculation including extensive lists of gas lines as well as dust. The disk is assumed to be geometrically thin (the height of the disk is smaller than its radius) which allows for the decoupling of radial and vertical disk structure. The disk is then divided into concentric rings and for each of these annuli the vertical structure and the detailed radiation field is calculated from the main input parameters, i.e. the stellar properties (mass, radius, effective temperature), mass accretion rate, and Reynolds number as a measure for the viscosity in the disk.

In this thesis the dependency of the models on the input parameters is demonstrated and first applications to three high-resolution infrared spectra of young stellar objects obtained with the ESO VLT instrument CRIRES are shown. For the CO emission line spectrum around $\lambda=4.7 \mu \mathrm{m}$ of GQ Lup, a well-fitting model could be calculated which allows to constrain the system's inclination to $i \approx 22^{\circ}$. Furthermore, the best fit model yields a mass accretion 
rate of $\dot{M}=3 \times 10^{-9} M_{\odot} \mathrm{yr}^{-1}$ and a Reynold number of $R e=5 \times 10^{4}$. For the much analysed star TW Hya, the known high energetic radiation field presumably produced in the accretion shock has to be accounted for as irradiation source in order to reproduce the observed line strength. Using this physically motivated additional emission pattern and a mass accretion rate of $\dot{M}=2 \times 10^{-9} M_{\odot} \mathrm{yr}^{-1}$, the inner radius of $R_{\text {in }}=0.09$ AU could be determined, which is in agreement with previous investigations. The spectrum of the classical T Tauri star RNO 90 shows very broad line wings and flat-topped emission peaks which cannot be reproduced by models which incorporate thermal microturbulence velocities. A better fit could be achieved by convolving the line profile with a Gaussian assuming some form of turbulent motion in the line emitting region of the order of the speed of sound in the disk.

In addition to the $1+1 \mathrm{D}$ disk model approach, the radiative transfer calculations were extended to three dimensions using the $3 \mathrm{D}$ radiative transfer framework of Hauschildt \& Baron. With this tool at hand two interesting problems were investigated. First, the effect of 3D radiative transfer on the line profile, especially in the case of non-axisymmetric disks, was studied. The change of the line profile for a disk with and without a density wave or an embedded planet was analysed. Assuming adiabatic density changes, a spiral arm in the inner disk $(R<0.2 \mathrm{AU})$ can be detected, while a small embedded planet in the same region will be harder to identify. Second, the effect of direct irradiation of the central star on the inner disk wall was investigated by means of iterative determination of the temperature structure from $3 \mathrm{D}$ radiative transfer simulations and the coupling to the 1+1D hydrostatic equilibrium condition and resulting opacities. It was found that direct stellar irradiation heats up the inner disk region substantially and the radial radiative transfer treatment leads to an overall temperature increase of the inner disk. Connected with a higher disk temperature is an overall hydrostatic blow-up of the disk. In none of the simulated cases the disk becomes completely optically thin despite the evaporation of dust in large parts of the disk. 


\section{Contents}

Contents viii

List of Figures $\quad x$

List of Tables $\quad$ xi

\begin{tabular}{lll}
\hline Introduction & 1
\end{tabular}

1.1 Young stellar objects . . . . . . . . . . . . . . . . . . . . . . . . 1

1.1 .1 Formation . . . . . . . . . . . . . . . . . . . . . . . 1

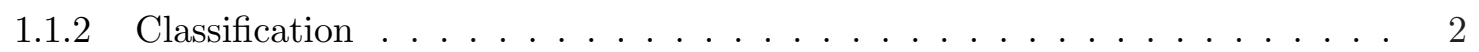

1.2 Circumstellar disks . . . . . . . . . . . . . . . . . . . . . . . . . 4

1.2 .1 Origin of IR excess $\ldots \ldots \ldots \ldots \ldots \ldots \ldots$

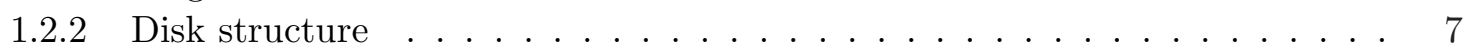

$1.3 \quad$ Inner disk regions . . . . . . . . . . . . . . . . . . . . . . . . . . . . . 9

1.3 .1 CO emission . . . . . . . . . . . . . . . . . . . 10

$1.3 .2 \mathrm{H}_{2}$ emission . . . . . . . . . . . . . . . . . . . 12

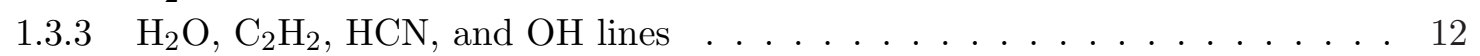

1.4 Disk model codes . . . . . . . . . . . . . . . . . . . . . . . . . . . . . 13

1.4 .1 Early models . . . . . . . . . . . . . . . . . . . . . 13

1.4 .2 Vertical structure models $\ldots \ldots \ldots \ldots \ldots$. . . . . . . . . . . . . . . . . . . . . . . . . . . . . . . .

1.4 .3 Multi-D codes . . . . . . . . . . . . . . . . . . . . . 14

1.5 Infrared spectroscopv $\ldots \ldots \ldots \ldots \ldots \ldots \ldots \ldots$

\begin{tabular}{|lll}
2 & $1+1 D$ models & 17
\end{tabular}

2.1 Standard accretion disk model . . . . . . . . . . . . . . . . . . . . 19

2.2 Radiation field . . . . . . . . . . . . . . . . . . . . . . . 20

2.3 Gas and dust properties . . . . . . . . . . . . . . . . . . . . . . 21

2.3.1 Occupation numbers . . . . . . . . . . . . . . . . . . . . . . . . . . . . . . . . .

2.3.2 Dust chemistrv and opacity . . . . . . . . . . . . . . . . . 22

2.4 Start models . . . . . . . . . . . . . . . . . . . . . . . 22

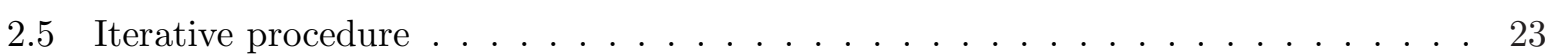

2.6 Hvdrostatic equilibrium . . . . . . . . . . . . . . . . . . . . . 24

2.7 Radiative transfer . . . . . . . . . . . . . . . . . . . 25

2.8 Irradiation geometrv . . . . . . . . . . . . . . . . . . . . 27

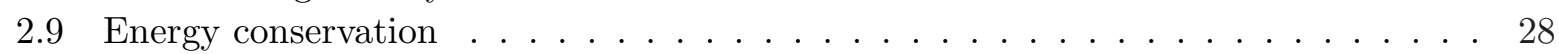

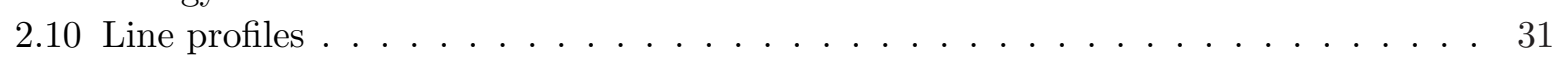

2.11 Parameter studies . . . . . . . . . . . . . . . . . . . 32 
2.11 .1 Mass accretion rate . . . . . . . . . . . . . . . . . . . . . 32

2.11 .2 Revnolds number . . . . . . . . . . . . . . . . . . . . . . . . . 35

2.11 .3 Stellar radius . . . . . . . . . . . . . . . . . . 37

2.12 Contribution function $\ldots \ldots \ldots \ldots \ldots \ldots$

$\begin{array}{lll}3 & \text { Spectral analvses } & 41\end{array}$

3.1 GQ Lup . . . . . . . . . . . . . . . . . . . . . . . . . . . 41

3.1 .1 Svstem parameters . . . . . . . . . . . . . . . . . . . . . . 41

3.1 .2 Model orid . . . . . . . . . . . . . . . . . . . . . . . . . . 42

3.1 .3 Inclination . . . . . . . . . . . . . . . . . . . . . . 43

3.1 .4 Model properties . . . . . . . . . . . . . . . . . . . . . . . . 44

3.1.5 Model vs. observation . . . . . . . . . . . . . . . . . . . . . 45

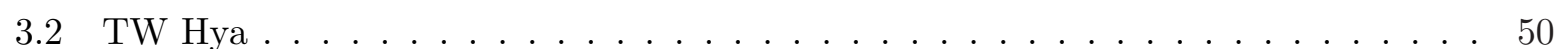

3.2 .1 Svstem parameters . . . . . . . . . . . . . . . . . . 50

3.2 .2 Model vs. observation . . . . . . . . . . . . . . . . . . . . 52

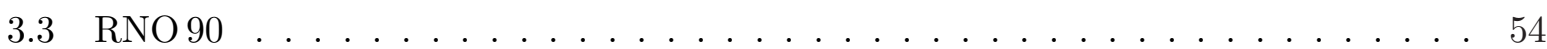

3.3.1 Svstem parameters . . . . . . . . . . . . . . . . . . 54

3.3.2 Model vs. observation . . . . . . . . . . . . . . . . . . . . 57

$\begin{array}{lll}4 & 3 \mathrm{D} \text { models } & 63\end{array}$

4.1 Radiative transfer . . . . . . . . . . . . . . . . . . . . 63

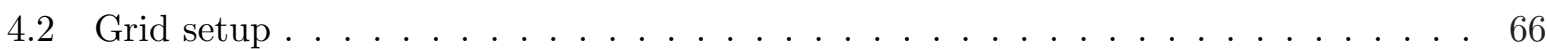

4.3 Line transfer in moving media . . . . . . . . . . . . . . . . . . . . . 68

4.3 .1 Grid resolution . . . . . . . . . . . . . . . . . . . . 70

4.3 .2 Axisvmmetric disks . . . . . . . . . . . . . . . . . . 72

4.3 .3 Non-axisvmmetric disks . . . . . . . . . . . . . . . . 76

4.4 Direct irradiation effects $\ldots \ldots \ldots \ldots$. . . . . . . . . . . . . . . . . 83

4.4 .1 Model approach . . . . . . . . . . . . . . . . . . 83

4.4 .2 Simulation results . . . . . . . . . . . . . . . . . 86

4.4 .3 Discussion $\ldots \ldots \ldots \ldots \ldots$. . . . . . . . . . . . . . . . . . . . . . . . . . . . 91

5 Summary and outlook $\quad 93$

\begin{tabular}{ll}
\hline Bibliography & I
\end{tabular}

$\begin{array}{ll}\text { Appendices } & \text { XI }\end{array}$

\begin{tabular}{|ll}
\hline A 3D line profile contributions & XI
\end{tabular}

A.1 Density wave . . . . . . . . . . . . . . . . . . . XII

A.2 Disk-planet model at $t=5$ orbits $\ldots \ldots \ldots \ldots \ldots$ XIII

A.3 Disk-planet model at $t=50$ orbits $\ldots \ldots \ldots \ldots \ldots$ XIV

B PHOENIX DISK mode $\quad$ XV

B.1 DISK input parameters . . . . . . . . . . . . . . . . XVV

B.2 DISK input files . . . . . . . . . . . . . . . . . . XVI 


\section{List of Figures}

$1.1 \quad$ Evolutionarv steps towards planetarv svstems . . . . . . . . . . . . . . . . . 2

1.2 HST images from protoplanetarv disks . . . . . . . . . . . . . . . . . . . 2

1.3 Spectral properties and their origins for Herbig Ae/Be stars . . . . . . . . . . . . 3

1.4 Evolution of stellar and disk mass and mass accretion rate . . . . . . . . . . . . . 4

1.5 Spectral energy distributions of voung stellar objects . . . . . . . . . . . . . . 5

1.6 Model SED of a flaring protoplanetary disk heated by stellar irradiation . . . . . . 6

1.7 Schematic dust and gas structure of a protoplanetarv disk . . . . . . . . . . . . 8

1.8 Number of inner gas radii of disks and orbital radii of short-period exoplanets . . . 9

1.9 Modelled CO fundamental emission band . . . . . . . . . . . . . . . . . . 10

1.10 Rotational diagram from $\mathrm{CO}$ lines $\ldots \ldots \ldots \ldots \ldots$. . . . . . . . . . . 11

1.11 CRIRES chip 1 for nodding positions $\mathrm{A}$ and $\mathrm{B} \ldots \ldots \ldots \ldots \ldots$

1.12 Reduction of CRIRES spectra . . . . . . . . . . . . . . . . . 16

$2.1 \quad$ Disk ring geometrv . . . . . . . . . . . . . . . . . . . . . . . . . 17

2.2 PHOENIX iteration scheme $\ldots \ldots \ldots \ldots \ldots \ldots \ldots$

2.3 Schematic sketch of a plane parallel disk atmosphere . . . . . . . . . . . . . . 21

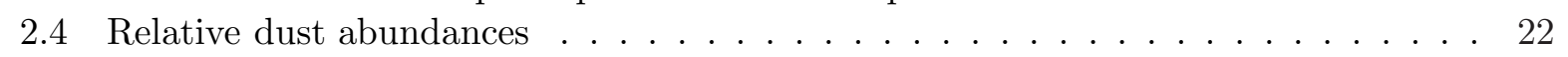

2.5 Irradiation geometrv as adopted for the model calculation . . . . . . . . . . . . 27

2.6 Temperature correction scheme . . . . . . . . . . . . . . . . . 29

2.7 Line broadening due to rotation of disk matter for a model line of Gaussian shape 31

2.8 Temperature profiles for disk rings with varving mass accretion rate . . . . . . . 32

2.9 Rosseland optical depth profiles for models with different mass accretion rates . . . 33

2.10 Density profiles for models with different mass accretion rates . . . . . . . . . . 33

2.11 Line profiles for the fundamental CO $v=1-0$ (R7) line calculated from models with different mass

2.12 Temperature profile for disk rings with varving Revnolds number . . . . . . . . . . 35

2.13 Rosseland optical depth profiles for models with different Revnold numbers . . . . 36

2.14 Density profiles for models with different Revnold numbers _ . . . . . . . . . . 36

2.15 Line profiles for the fundamental CO $v=1-0$ (R7) line from models with different Revnolds numbe

2.16 Temperature profile for disk rings with varving stellar radius $\ldots \ldots \ldots$. . . . 38

2.17 Line profiles for the fundamental CO $v=1-0$ (R7) line from models with different stellar radii 38

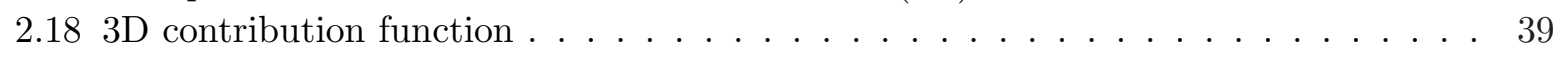

2.19 Line and continuum contribution function . . . . . . . . . . . . . . 40

3.1 Comparison between observed CO line profile and models with $i=22^{\circ}$ and $i=51^{\circ} \quad 43$

3.2 Line contributions of ring models . . . . . . . . . . . . . . . . . 44

3.3 Temperature structures for different Revnolds numbers $R e \ldots \ldots \ldots$

$3.4 \mathrm{CO}$ spectrum for different Revnolds numbers $R e \ldots \ldots \ldots$ 
3.5 Spectra calculated from the same structure model but for different dust grain sizes 47

3.6 CRIRES spectrum of GQ Lup with the best-fit disk model . . . . . . . . . . . . . 48

3.7 CRIRES spectrum of GQ Lup with the best-fit disk model . . . . . . . . . . . . . . 49

3.8 Comparison of TW Hva model irradiation sources . . . . . . . . . . . . . . . . 51

3.9 Temperature structures for different irradiation source treatments . . . . . . . . . . 51

3.10 CRIRES spectrum of TW Hva with the best-fit disk model . . . . . . . . . . . 53

3.11 Temperature and surface densitv over radius for DISK model and power law description 54

$3.12 \mathrm{CO}$ emission and absorption spectrum of RNO $90 \ldots \ldots \ldots \ldots \ldots$

$3.13{ }^{12} \mathrm{CO}$ and ${ }^{13} \mathrm{CO}$ absorption line fits for RNO $90 \ldots \ldots \ldots \ldots \ldots$

3.14 CRIRES spectrum of RNO 90 compared to a disk model . . . . . . . . . . . . 58

3.15 CRIRES spectrum of RNO 90 with the best-fit disk model . . . . . . . . . . . . . 59

3.16 Co-added $\mathrm{CO}$ emission line profile of RNO $90 \ldots \ldots \ldots$. . . . . . . . . 60

4.1 Schematic plots of different tvpes of characteristic . . . . . . . . . . . . . . 64

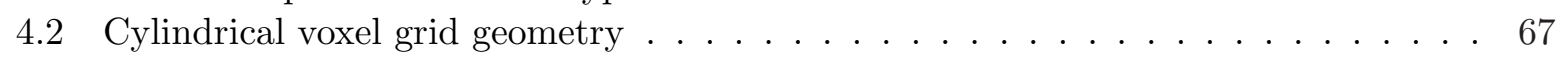

4.3 Surface plot of disk temperatures . . . . . . . . . . . . . . . . . . . 68

4.4 Visualisation of grid resolution effects $\ldots \ldots \ldots \ldots \ldots \ldots$

4.5 Comparison of temperature structure resolutions . . . . . . . . . . . . 71

4.6 3D line profiles for disk structures with different line thermalisation parameter . . 72

4.7 Difference between 3D spectral lines emitted from disk surface and total disk . . . 73

4.8 Run of the line-integrated mean intensitv with radius . . . . . . . . . . . . 73

4.9 Illustration of disk emission regions considered for line profiles . . . . . . . . . . . 74

4.10 Comparison between 1D and 3D line profiles . . . . . . . . . . . . 75

4.11 Line profiles for a disk with a densitv wave . . . . . . . . . . . . . . . . 77

4.12 Density contour plots for a disk with embedded planet . . . . . . . . . . . . . 78

4.13 Run of the density with radius for a disk with embedded planet . . . . . . . . . . 79

4.14 Radial surface densitv and temperature structure for a disk with an embedded planet 79

4.15 Continuum intensity plots for disk-planet models . . . . . . . . . . . . . . 80

4.16 Line profiles for disk-planet scenarios . . . . . . . . . . . . . . . . . . . . . 81

4.17 Disk opacities . . . . . . . . . . . . . . . . . . . . . . . . 84

4.18 Flow chart of $3 \mathrm{D}$ structure calculations . . . . . . . . . . . . . . . . 85

4.19 3D temperature structures for disk setup I . . . . . . . . . . . . . . . . 86

4.20 Midplane temperature history for disk setup I . . . . . . . . . . . . . . . 87

4.21 Midplane temperature historv for disk setup II $\ldots \ldots \ldots \ldots$

4.22 Temperature contour plot for disk setup II . . . . . . . . . . . . . . . . . . 89

4.23 Midplane temperature historv for disk setup III . . . . . . . . . . . . . . . . 90

4.24 Temperature contour plot for disk setup III . . . . . . . . . . . . . . . . . . . . . . . 91

A.1 Line contributions for the disk with density wave . . . . . . . . . . . . . . . . XII

A.2 Line contributions for the $t=5$ orbits planet-forming disk $\ldots \ldots \ldots$. . . . XIII

A.3 Line contributions for the $t=50$ orbits planet-forming disk . . . . . . . . . . XIV 


\section{List of Tables}

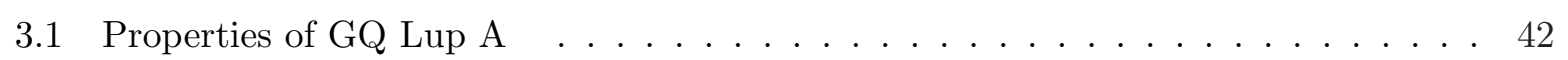

3.2 CO absorption line parameters for RNO $90 \ldots \ldots \ldots \ldots \ldots$ 



\section{Meinen Eltern}





\section{Chapter 1}

\section{Introduction}

Stars and interstellar material can be observed because they radiate. In order to interpret the radiation that we can detect on Earth or with satellites, radiative transfer models of the objects observed are needed. The more detailed the models are, the stronger the constraints that we can draw. Hence, modern astrophysical research demands the most detailed models that are computationally bearable. This is especially true because theory needs to keep up with the immense progress made on the observational side. The formation of stars is occurring in our own but also in galaxies far away. Planets are formed along with stars from the same material in a circumstellar disk. This process is essential for a complete understanding of our universe. In this thesis models for circumstellar, in particular protoplanetary, disks will be described and the results of model calculations will be compared to spectroscopic observations.

\section{$1.1 \quad$ Young stellar objects}

\subsubsection{Formation}

Astronomical observations support the picture that stars form from large and massive interstellar clouds. In order to start such a formation process, certain criteria have to be fulfilled. Generally speaking, an interstellar dust or gas cloud will collapse once its own gravity exceeds the internal gas pressure. The thermodynamical conditions for such an event are described by the Virial theorem and instability occurs when

$$
2 E_{\text {therm }}+E_{\text {grav }}<0
$$

Assuming a constant density, instability is described by the Jeans-criterion:

$$
G \frac{M}{R}>\frac{5 k_{B} T}{\bar{\mu} m_{\mathrm{H}}}
$$

where $k_{B}$ is the Boltzmann constant and $\bar{\mu}=\bar{m} / m_{\mathrm{H}}$ the mean mass. Depending on the type of cloud the necessary mass and radius can be estimated if density $\varrho$, temperature $T$, and mean mass $\bar{\mu}$ are known. For a typical cool molecular cloud with $\varrho_{\mathrm{H}_{2}}=50 \mathrm{~cm}^{-3}, T=20 \mathrm{~K}$, and $\bar{\mu}=2$ the Jeans mass is $M_{\mathrm{J}}=2500 M_{\odot}$ and the Jeans radius $R_{\mathrm{J}}=4$ pc. Since stars with masses $>100 M_{\odot}$ have never been observed (except for the very first stars in the universe), the clouds must fragment during collapse. This can also be explained by the conversion of potential energy into radiation during the contraction process. At the beginning of the contraction phase 


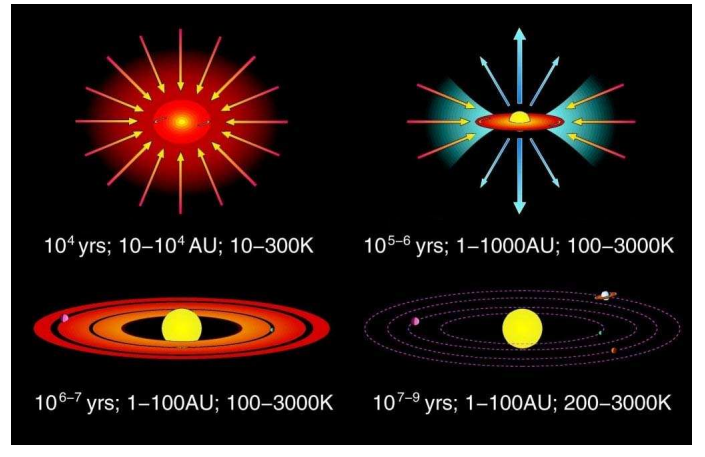

Figure 1.1: Evolutionary steps from a collapsing gas and dust cloud to a planetary system. For each graphic a time scale, a dimension, and a temperature range is given (taken from Gardner et al., 2006).

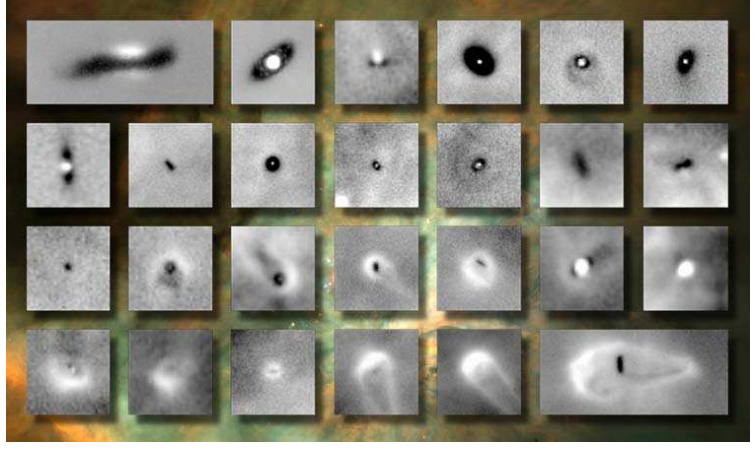

Figure 1.2: HST images of protoplanetary disks in the Orion Nebula (taken from Gardner et al., 2006).

radiation can escape quite efficiently and hence no significant heating occurs. With increasing density, the Jeans mass decreases and gravitational collapse continues on smaller scales. Once matter becomes optically thick, heating becomes more efficient and the fragments warm up. As a result, the Jeans mass increases and further fragmentation is stopped. If a protostellar cloud is rapidly spinning it may split up into multiple systems.

Each protostar will contract faster in the center and pressure increases due to the heating of the gas. Contraction will slow down in the core while matter is still infalling freely at larger radii. Subsequent consumption of internal energy due to $\mathrm{H}_{2}$ dissociation and hydrogen and helium ionisation will lead to a slower increase in temperature and pressure and hence contraction speeds up. Contraction of the protostar will come to an end once the bulk of matter is completely ionised.

Initial random motion of the gas will follow the net angular momentum of the nebula. Eventually, a disk forms around the protostar due to the conservation of angular momentum. It possesses $\sim 99 \%$ of the system's angular momentum but only a few percent of its mass. The infall phase lasts about $10^{5}$ years. The young stellar object (YSO) will continue to accrete matter through the disk for about $10^{7}$ years until the disk disappears due to e.g. a strong stellar wind or simply a lack of material. For an illustration of the planetary system formation scenario see Fig. 1.1. Observations of stars in the Orion Nebula embedded in their dust and gas cloud of formation is shown in Fig. 1.2

\subsubsection{Classification}

Depending on their mass, YSOs can be divided into two groups: T Tauri stars (TTS) and Herbig Ae/Be stars. The former have masses below $2 M_{\odot}$ corresponding to spectral types F, G, K, and $\mathrm{M}$ while the latter are more massive with 2-10 $M_{\odot}$ and spectral types A or B. Tools for studying Herbig Ae/Be stars are shown in Fig. 1.3.

TTS have masses and effective temperatures similar to main sequence stars but they have not contracted to their final main sequence radii yet. Therefore, they are more luminous than their main sequence counterparts. In the Hertzsprung-Russell diagram (HRD) TTS populate the regions of fully convective and partially radiative pre-main sequence (PMS) stars (see e.g. Cohen \& Kuhi, 1979). Their overall spectral energy distribution typically shows an IR and UV 


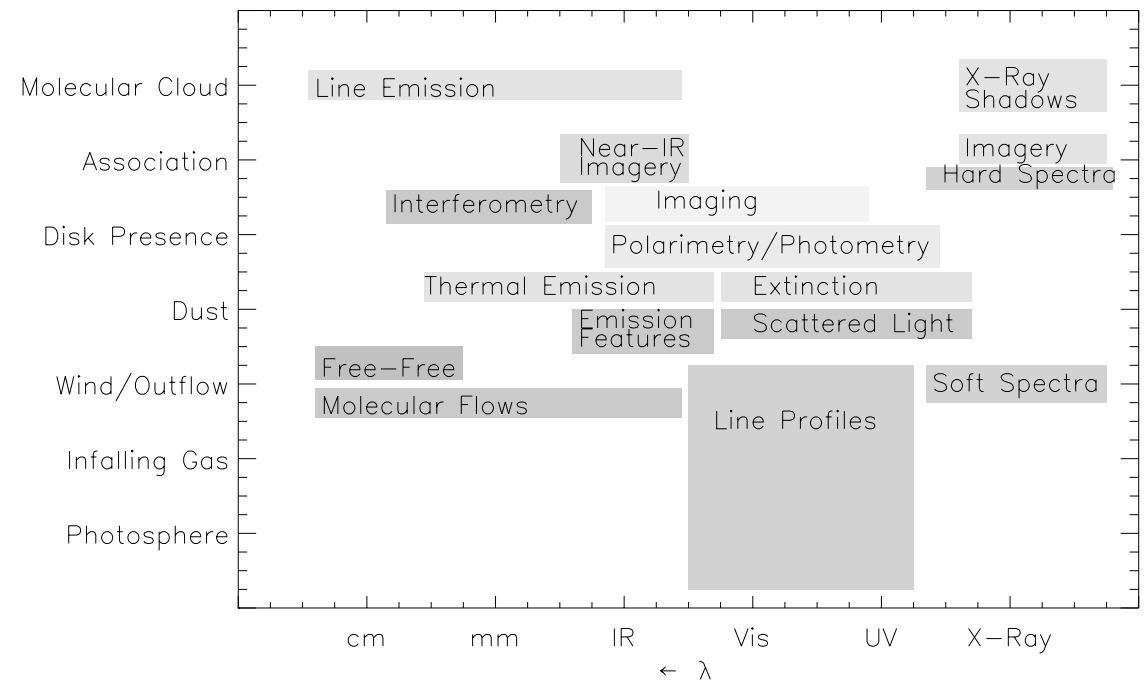

Figure 1.3: Spectral properties and their origins for Herbig Ae/Be stars (taken from Perez \& Gradv, 1997).

excess compared to the main sequence counterparts. The IR emission can be attributed to a surrounding gas and dust disk. These TTS also have stronger sub-mm emission. The optical spectra are described to have a stellar continuum and absorption line spectrum with a superimposed non-photospheric continuum, and an emission line spectrum (e.g. Appenzeller \& Mundt, 1989, and references therein). The absorption spectrum is similar to that of main-sequence dwarf stars (spectral type $\mathrm{K}-\mathrm{M}$ ) with the exception that Li I is much stronger since it its not yet destroyed by proton-capture reactions (e.g. Bodenheimer, 1965). Due to the superimposed emission lines and the non-photospheric continuum, photospheric absorption lines in TTS are weaker than in main sequence stars of similar temperatures. This effect is called "veiling". Barrado v Navascués \& Martín (2003) provide empirical criteria to classify TTS.

$\mathrm{T}$ Tauri stars can be split into subgroups depending on their spectral appearance. A classical T Tauri star (CTTS) shows an emission line spectrum including hydrogen Balmer (especially a strong $\mathrm{H} \alpha$ ) lines, He I, CaII H and K, as well as Ca II infrared triplet at 8498, 8542, and $8662 \AA$. The emission lines are broad and sometimes asymmetric; especially $\mathrm{H} \alpha$ can have some hundred $\mathrm{km} \mathrm{s}^{-1}$ at FWHM. Furthermore, narrow and generally blue-shifted forbidden lines of e.g. O I, N II, or S II are characteristic for CTTS. An additional feature of CTTS is the above mentioned UV and IR continuum excess. A simple criterion to identify a CTTS is by its equivalent width of the $\mathrm{H} \alpha$ line, i.e. $W(\mathrm{H} \alpha)>5 \AA$. The broad hydrogen Balmer lines as well as the continuum excess can be explained by the surrounding disk. The relatively cool circumstellar disk is the origin of the IR excess, while the UV excess and the broad Balmer lines stem from the process of mass accretion onto the star. Disk matter is channeled onto the star along strong magnetic field lines. The material that hits the stellar surface is heated up in a hot spot and creates the UV excess. The broad hydrogen lines originate in the material transported from the disk onto the star. The narrow forbidden lines are formed in outflowing jets which are caused by the accretion. X-ray emission has been observed for CTTS and is believed to form in the shock region and additionally in the corona (Günther et al., 2007).

In contrast to the rich spectrum of CTTS, weak-line T Tauri stars (WTTS) rather lack strong spectral signatures. They also show $\mathrm{H} \alpha$ in emission but less broad with $W(\mathrm{H} \alpha)<5 \AA$ 


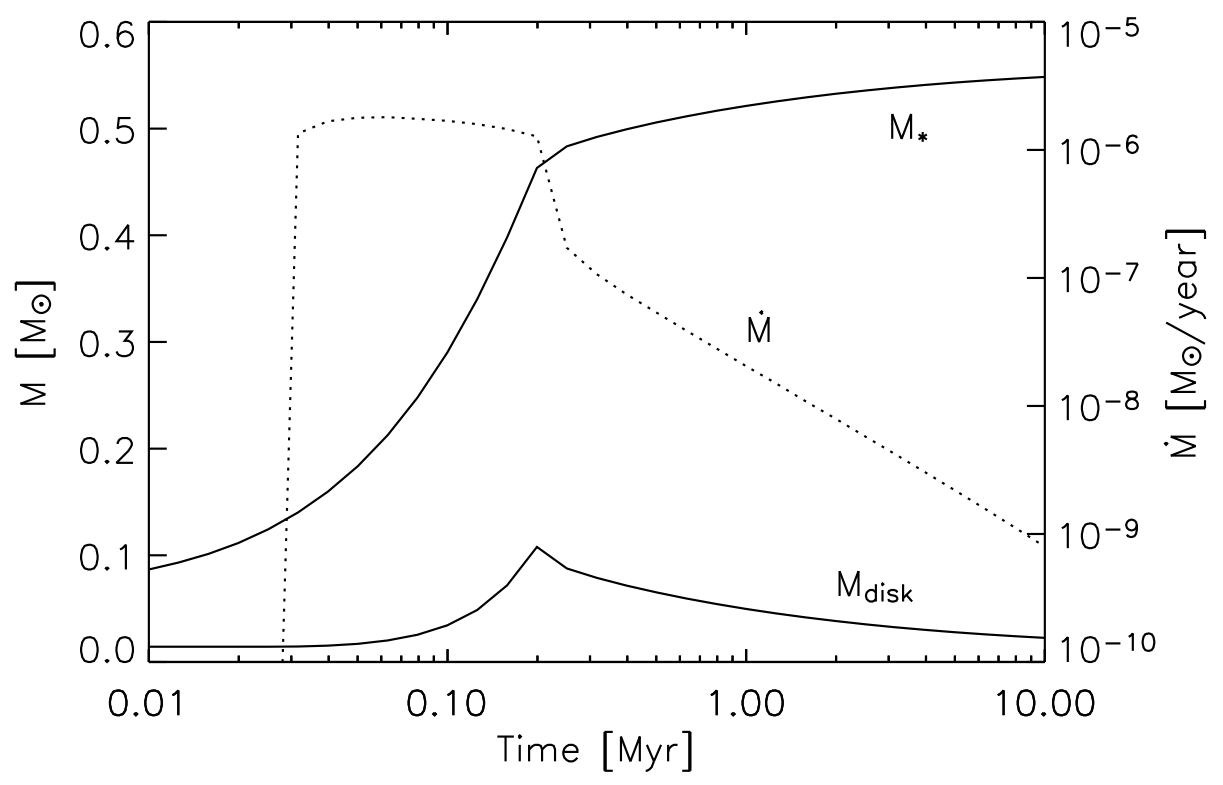

Figure 1.4: Evolution of stellar and disk mass and mass accretion rate with time (taken from Dullemond et al., 2007). In the model from Hueso \& Guillot (2005) the disk forms at $t \sim 0.03 \mathrm{Myr}$ and the collapse phase ends at $2 \times 10^{5} \mathrm{yr}$.

(Herbig \& Bell, 1988). Also the UV excess is not present and "veiling" is missing. The IR excess is, if at all, only weakly present. Therefore, WTTS are believed to be more evolved PMS stars with no or only residual circumstellar material left.

The more massive Herbig Ae/Be (HAeBe) stars are also characterised by Balmer emission lines and IR excess due to circumstellar disks. The latter distinction is important since classical Be stars show IR excess caused by free-free emission. HAeBes also show UV excess as a result of accretion and IR excess which, however, is less intense than for CTTS (Waters \& Waelkens, 1998). Furthermore, X-rays have been detected from HAeBes stars by e.g. Hamaguchi et al. (2005), who propose an origin from magnetic activity. Alternatively, the X-rays may originate from a TTS companion.

\subsection{Circumstellar disks}

Circumstellar disks are complex systems and their properties vary strongly with radius. Many effects such as stellar magnetic fields or disk winds influence the structure and evolution of protoplanetary disks. The chronological evolution of the central star and disk mass and mass accretion rate is shown in Fig. 1.4. The model calculations of Hueso \& Guillot (2005) predict a disk formation around $t \sim 0.03 \mathrm{Myr}$ and an initial high mass accretion rate of $\sim 10^{-5} M_{\odot} \mathrm{yr}^{-1}$. After this first phase of rapid star and disk formation around $t \sim 0.20 \mathrm{Myr}$, the mass accretion rate drops quickly down to $10^{-7} M_{\odot} \mathrm{yr}^{-1}$ from where it will decrease to $10^{-9} M_{\odot} \mathrm{yr}^{-1}$ within $10 \mathrm{Myr}$. During that time, the stellar mass slowly increases and the disk mass, since there is a limited reservoir of matter, diminishes.

Protoplanetary disks were originally classified (Lada \& Wilking, 1984; Lada, 1987) according 

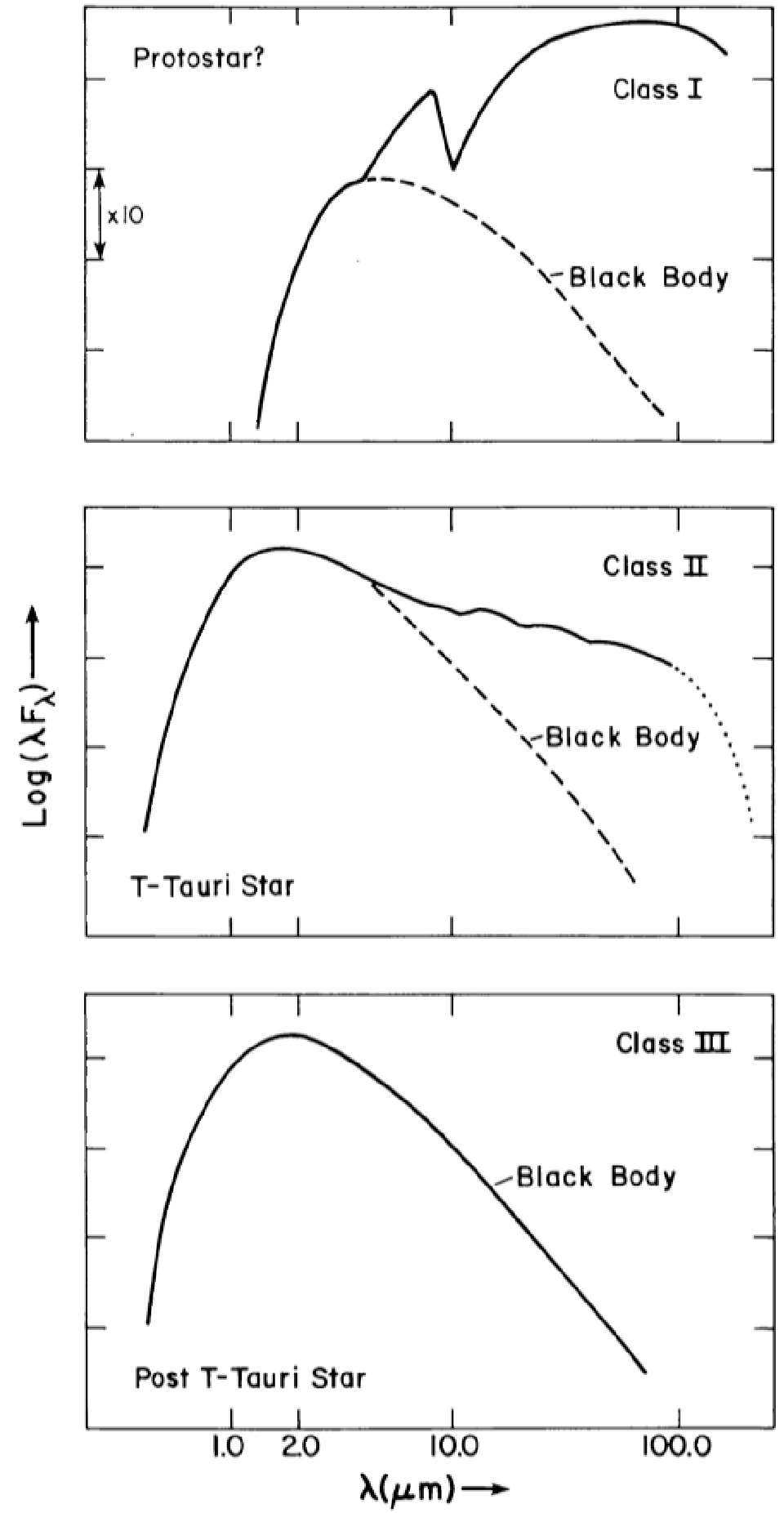

Figure 1.5: Spectral energy distributions of young stellar objects (taken from Lada, 1987). Class I objects show a strong IR excess and the (proto-)star is not yet visible. The older class II objects have a flatter SED in the IR and show a combination of stellar and disk spectrum. Class III objects can be modelled by a blackbody of stellar effective temperature. 


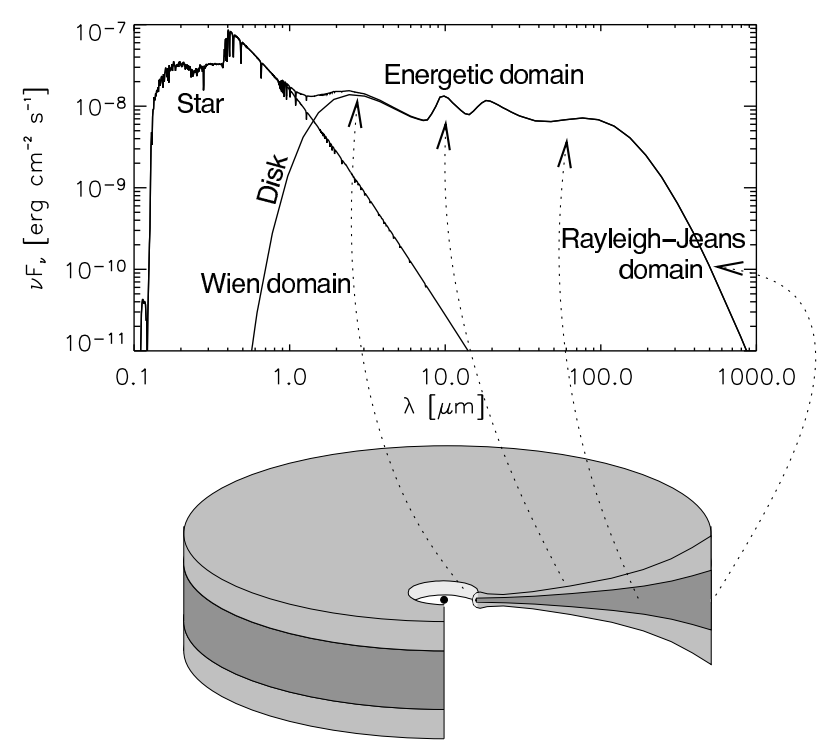

Figure 1.6: Model SED (without scattering) of a flaring protoplanetary disk heated by stellar irradiation (taken from Dullemond et al., 2007). The near-IR emission is assigned to the warm inner disk regions, the mid-IR features are emitted from the warm surface layers, and the underlying continuum comes from the cooler regions closer to the midplane of the disk. The sub-mm emission typically originates in the inner regions of the outer disk.

to their spectral index

$$
s=\frac{\mathrm{d} \log \left(\lambda F_{\lambda}\right)}{\mathrm{d} \log (\lambda)}
$$

into the following classes:

$\begin{array}{lr}\text { class I: } & 0<s \leq 3 \\ \text { class II: } & -2 \leq s \leq 0 \\ \text { class III: } & -3<s \leq-2\end{array}$

Class I objects are sources with spectral energy distributions (SEDs) broader than expected from a blackbody of the stellar effective temperature. The flux is even rising longward of $2 \mu \mathrm{m}$. These SEDs come from very young objects which are deeply embedded in the cloud from which they formed. Class II objects also show a broader than blackbody SED, however, the flux is either flat red of $2 \mu \mathrm{m}$ or even decreasing. In the case of class III objects, the SEDs can be modelled with reddened blackbodies. Objects of the latter two classes can usually be associated with stars visible in the optical light. SEDs for the three classes are shown in Fig. 1.5 Andre et al. (1993) later added the additional class 0 which is mainly characterised by sub-mm excess emission. These objects are in an even earlier evolutionary stage than the class I objects.

\subsubsection{Origin of IR excess}

The origin of the emitted IR and sub-mm flux in class I and II objects can be attributed to different regions in the disk which is surrounding the YSO. Most of the emitted flux comes from 
the thermal emission of dust which is by a factor of $\sim 100$ (e.g. Karttunen et al., 2003) less abundant than gas but has a much higher opacity. The light scattered on dust grains in the disk also contributes to the observed emission. Figure 1.6 shows a model SED of a star with a circumstellar disk as well as the origin of the emitted flux in a disk model. The energetic domain spans a range from $\sim 1 \mu \mathrm{m}$ to $\sim 100 \mu \mathrm{m}$ and is set by the minimum and maximum dust temperature in the disk. The near-IR bump is assigned to the very inner region of the disk. The temperature there is especially high due to the proximity to the star. The reason for this thermal emission has been discussed controversially. Lynden-Bell \& Pringle (1974) suggested that the IR emission is generated from accretion processes in the disk. In that case a flat and steady state disk is heated by viscous dissipation due to turbulent motion of matter in the disk - a process essential for mass and angular momentum transport. Alternatively, a perfectly flat disk could be heated due to the absorption of stellar radiation. Adams \& Shu (1986) and Adams et al. (1987b) determined that the dependence of disk temperature on radius is similar for accretion and passive irradiated disks, i.e. $T(R) \propto R^{-3 / 4}$. Therefore, it is difficult to specify the origin of the IR excess from the spectral energy distribution for these two models. A disk with a significant accretion rate is likely to be optically thick (Lvnden-Bell \& Pringle, 1974) and hence reprocessing of stellar light will take place.

Kenvon \& Hartmann (1987) show that the IR excess of most TTS can be explained by reprocessed stellar radiation. The simple flat disk model predicts that at most $25 \%$ of the stellar light will be absorbed and re-emitted by the disk (Adams et al., 1987b). For many objects the flux level rather correspond to an absorption of $50 \%$ of the emitted stellar light. Hence, Kenvon \& Hartmann (1987) proposed a flaring instead of a flat disk which allows to absorb more star light at large radii, thus heating these cool disk areas and increasing the mid- to far-IR emission.

Assuming that reprocessed stellar irradiation is the only source of the observed disk luminosity, a simple estimate for a dust grain temperature can be made by equating the absorbed stellar flux by the projected grain surface area with the re-emitted thermal flux of the grain surface:

$$
\pi a^{2} \frac{L_{\star}}{4 \pi R^{2}}=4 \pi a^{2} \sigma T^{4} \Longleftrightarrow T=\left(\frac{L_{\star}}{16 \pi \sigma R^{2}}\right)^{1 / 4}
$$

Hence, independent of grain size, the temperature for a dust particle which is living in the surface of the disk atmosphere and is thus not shielded but directly irradiated by the central star can be computed. For a typical TTS with $R_{\star}=2 R_{\odot}$ and $T_{\text {eff }}=4000 \mathrm{~K}$ (Kenvon \& Hartmann, 1987) the temperature at an inner dust radius of $0.1 \mathrm{AU}$ is $T_{\text {grain }}=863 \mathrm{~K}$ which corresponds to a maximum blackbody flux at a wavelength of $\lambda_{\max }=4 \mu \mathrm{m}$. The minimum inner dust radius of a circumstellar disk is determined by the dust sublimation temperature of $\sim 1500 \mathrm{~K}$ (Pollack et al., 1994). For the typical TTS described above, the inner dust rim is at $R \approx 0.03 \mathrm{AU}$ and most of the energy is radiated at $\lambda_{\max }=2 \mu \mathrm{m}$.

\subsubsection{Disk structure}

The structure of protoplanetary disks is very complex and only partially understood. The composition of a disk depends strongly on age and other outside influences, e.g. the amount of matter available or nearby luminous $\mathrm{O}$ stars that blow away disk matter. Axial asymmetries can be present which make an analysis of such a disk even more difficult. In the following description of the disk structure, a symmetric and "typical" representation of a disk is used. Schematic plots of the dust and gas structure of a disk are shown in Fig. 1.7. 
Dust-structure of disk

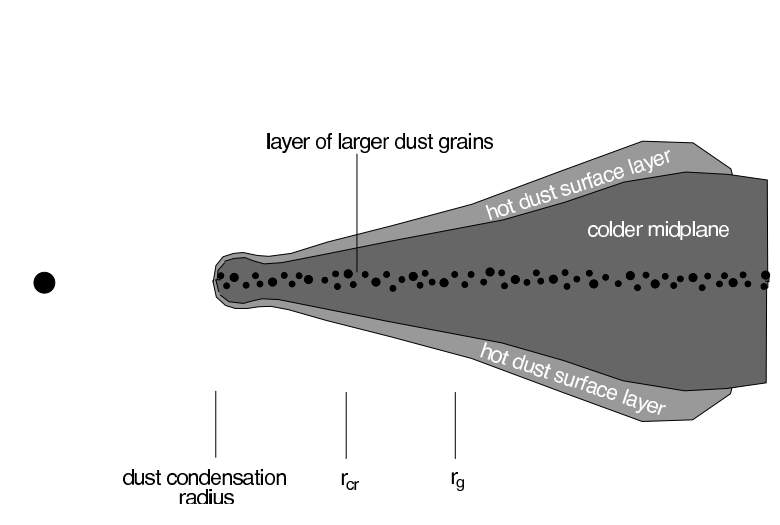

Gas structure of disk

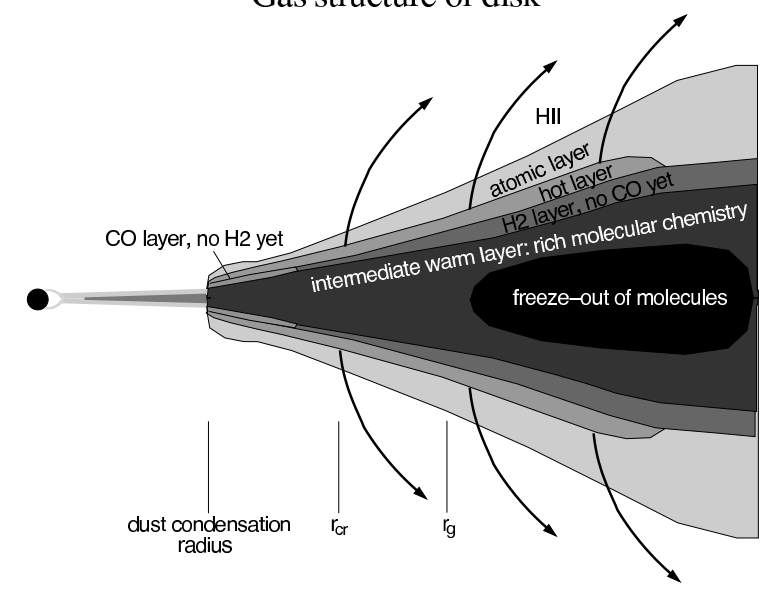

Figure 1.7: Schematic dust (left) and gas (right) structure of a protoplanetary disk. Regions of different physical and chemical conditions are shown. The arrows denote winds that are driven by FUV and EUV irradiation (taken from Dullemond et al., 2007).

Since protoplanetary disks form from interstellar material (ISM) it can be assumed that 99\% of the disk mass is in gas and only $1 \%$ in dust (e.g. Karttunen et al., 2003). However, the dust plays an important role for the disk observability because it scatters and thermally emits radiation that we can observe as IR excess (see previous Section). Dust is also important for setting the thermal structure of disks because of its dominating opacity over the gas content (Natta et al., 2007). Therefore, the gas is shielded by the dust from stellar irradiation and the ionisation fraction is reduced. Furthermore, dust grains are very important for chemical reactions and freeze-out of molecules. Additionally, coagulation and fragmentation leads to growth of solid particles which are believed to be the origins of planet formation (e.g. Brauer et al., 2008). The vertical stratification of dust that forms in the disk will be affected by two competing mechanisms. Gravity forces the dust to settle towards the midplane while turbulent diffusion mixes the dust and transports it back to higher layers in the disk.

The dust disk is truncated at the dust condensation radius $R_{\mathrm{dc}}$ which is the distance from the star where the dust temperature reaches $\sim 1500 \mathrm{~K}$, i.e. the point where dust thermally dissociates . For a CTTS with $R_{\star}=2 R_{\odot}$ and $T_{\text {eff }}=4000 \mathrm{~K}$ the inner dust radius is located at $R_{\mathrm{dc}} \approx 0.03 \mathrm{AU}$ and for a HAeBe star of $R_{\star}=5 R_{\odot}$ and $T_{\mathrm{eff}}=10000 \mathrm{~K}$ at $R_{\mathrm{dc}} \approx 0.50 \mathrm{AU}$ using the approximative equation (1.4). The shape of the inner dust rim will depend on the material inside $R_{\mathrm{dc}}$. Muzerolle et al. (2004) calculated models to answer the question how much stellar irradiation is blocked by the gas inside $R_{\mathrm{dc}}$. They assumed a typical HAeBe star and different mass accretion rates of $\dot{M}=10^{-9}, 10^{-8}, 10^{-7}$, and $10^{-6} M_{\odot} \mathrm{yr}^{-1}$. In all cases, the inner dust wall receives large fractions of stellar irradiation $(\sim 1,0.9,0.8$, and 0.7 for the accretion rates given above), which means that the portion of the dust wall that is in contact with the inner optically thick gaseous disk is small. Hence, the very inner dust part of the disk is irradiated at a $\sim 90^{\circ}$ angle while dust at larger radii is irradiated at much shallower angles and only the topmost layers of the disk see star light. As a consequence, the dust near $R_{\mathrm{dc}}$ is much warmer probably vertically isothermal, leading to an increase in height as a result of hydrostatic equilibrium. The inner rim is said to be "puffed-up" and is a natural explanation for the near-IR bump seen in many HAeBe SEDs (Natta et al., 2001).

The very outer disk is not resupplied with matter after the infall phase is over. Due to accretion 


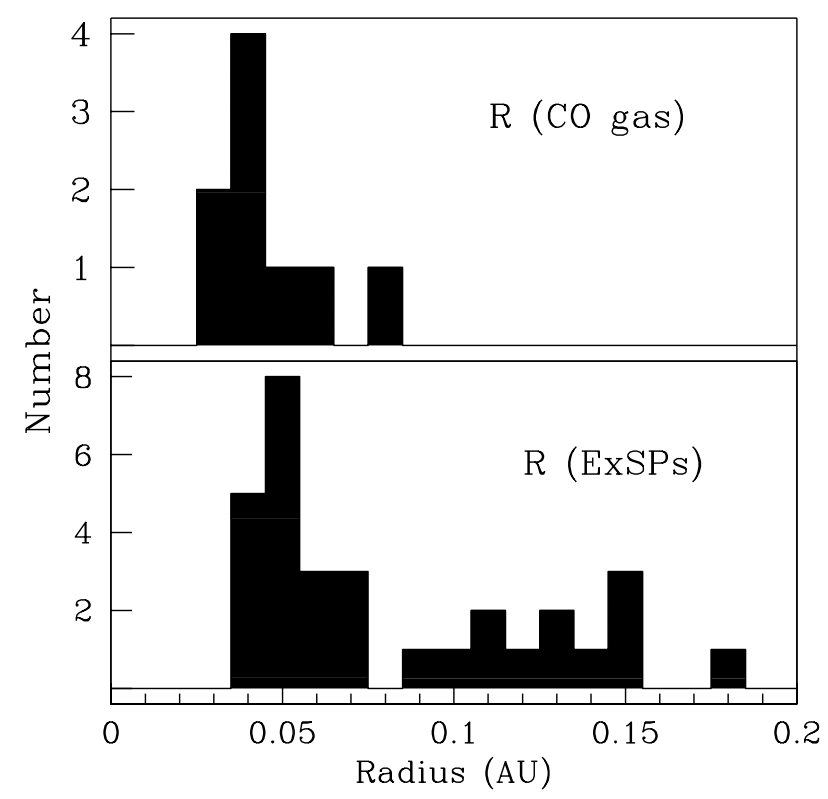

Figure 1.8: Number of inner gas radii measured from $\mathrm{CO}$ lines in protoplanetary disks (top) and orbital radii of short-period exoplanets (bottom). The distributions look very similar even though the number of measured radii is small (taken from Najita et al., 2007).

of matter onto the star and photoevaporation the disk will eventually drain (see Fig. 1.4). The viscous time scale for irradiated disks is $t_{\text {vis }} \propto r$, hence, the outer parts evolve the slowest and serve as a mass reservoir for the inner regions.

The gas structure of disks is shown in the right panel of Fig. 1.7. The matter that is accreted onto the star will be in the form of gas because all dust grains are thermally dissociated. The gas is believed to follow the magnetic field lines which extend out to a few stellar radii (e.g. Shu et al., 1994). The position where disk material has the same orbital velocity as the rotation of the star is referred to as the corotation radius. The mechanism of stellar magnetic field and disk interaction is often called "disk locking". The strength of mass accretion onto the central star is commonly evaluated by the full width of the $\mathrm{H} \alpha$ line at $10 \%$ of the peak (e.g. Javawardhana et al., 2006). The line is dominated by the infalling gas from the disk. An extra contribution comes from chromospheric activity. A threshold $10 \%$ width of $\sim 200 \mathrm{~km} \mathrm{~s}^{-1}$ has been introduced to divide between accretors and non-accretors (Javawardhana et al., 2003) which corresponds to an accretion rate of $\dot{M}=10^{-11} M_{\odot} \mathrm{yr}^{-1}$ (Natta et al., 2004). Often stellar winds lead to absorption features in the $\mathrm{H} \alpha$ line. An older stellar accretion model assumed that the gas reaches all the way to the star and forms a small and hot boundary layer, however, there were problems to explain the relatively small rotation velocities observed in CTTS.

In the top strongly irradiated layers of protoplanetary disks densities are very small and the physics and chemistry there are similar to that in photon dominated regions (Tielens \& Hollenbach, 1985; Yamashita et al., 1993). Because of the small densities gas and dust have small collision rates and hence are not in thermal equilibrium. Calculations of the thermal balance of all relevant gas heating and cooling mechanisms (e.g. Woitke et al., 2009) show that the temperature of the gas clearly exceeds the dust temperature. This is valid for densities $n<10^{5} \mathrm{~cm}^{-3}$ (Kamp \& Dullemond, 2004). Due to the high temperatures, H I will evaporate in the upper 


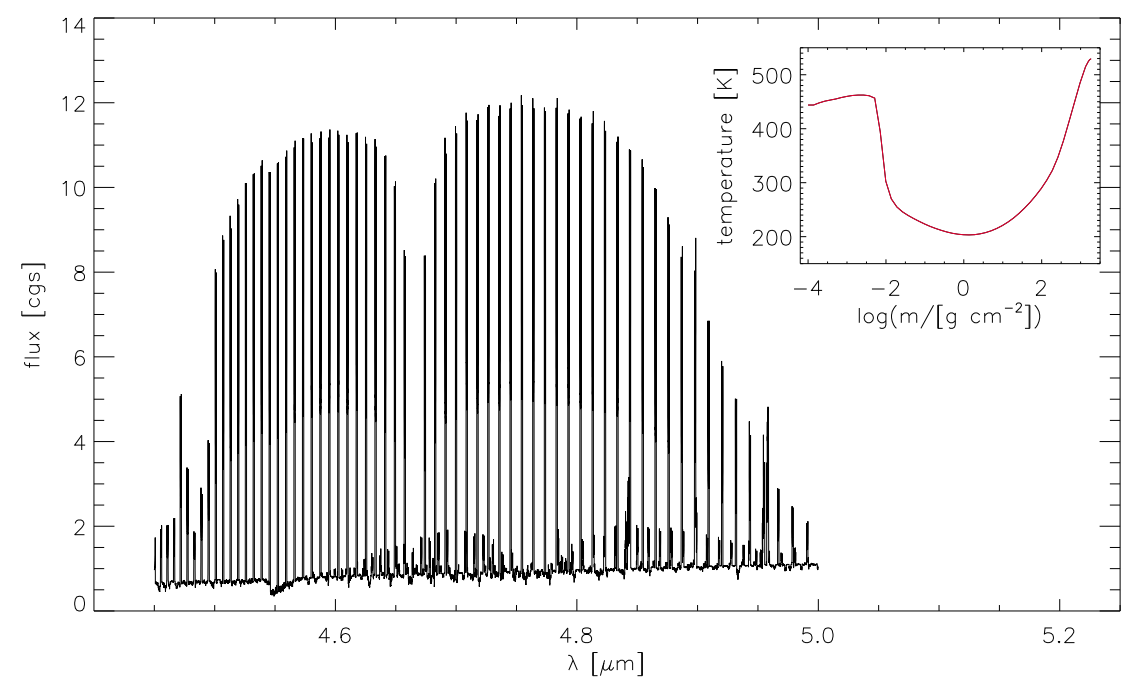

Figure 1.9: Modelled CO fundamental emission band for a typical protoplanetary disk annulus with $R=0.29$ AU seen under an inclination of $i=40^{\circ}$. The small plot in the upper right corner shows the vertical temperature structure for the disk atmosphere.

layers since its thermal velocity exceeds the escape velocity. Kamp \& Dullemond (2004) give a conservative lower temperature limit for photoevaporation of

$$
T_{\mathrm{esc}}=\frac{G M_{\star} m_{\mathrm{H}}}{k_{\mathrm{B}} R} .
$$

In the denser layers deeper in the atmosphere higher collision rates of dust and gas lead to a temperature balance.

\subsection{Inner disk regions}

The inner regions of protoplanetary disks are of special interest due to various reasons. First of all, the gas and dust within $R \lesssim 10 \mathrm{AU}$ of the central star is believed to be the material from which planets form. Therefore, the structure and composition will determine the properties of planets born in the disk. The distribution of inner gas disk radii and radii of short-period exoplanets shown in Fig. 1.8 supports the picture of halted migration due to disk truncation. Furthermore, the lifetime of gas in the disk sets an upper limit to the giant planet formation timescale. The inner regions are particularly interesting for the diagnostics of young stellar systems because the material is warm enough $(100 \mathrm{~K}$ to $1500 \mathrm{~K})$ for the formation of various molecular and atomic spectral lines, hence, a direct diagnostic tool for the investigation of the abundant gas is available. The spectral lines often appear in emission which can be explained by i) an optically thin $(\tau<1) 1$ slab of emitting material (no optically thick continuum) or ii) an vertically optically thick $(\tau>1)$ disk with a temperature inversion in the outer layers, i.e. the temperature rises as one goes to smaller optical depths. The second scenario is probably the more common one in CTTS while the first can occur when the column density is reduced by dynamical clearing effects or dissipation of the disk.

\footnotetext{
${ }^{1} \tau(z)=\int_{z}^{\infty} \kappa d z^{\prime}$, with $\kappa$ the opacity
} 

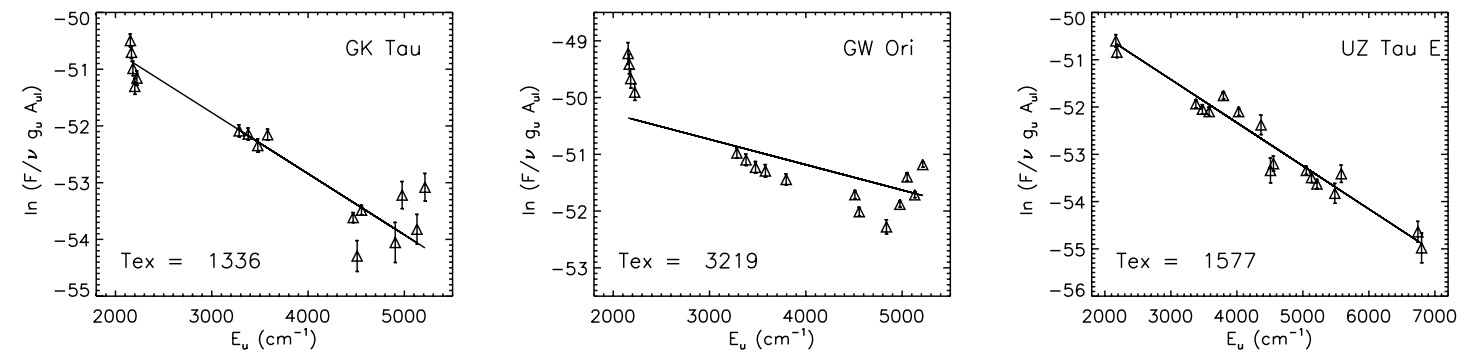

Figure 1.10: Rotational diagram from CO lines for different objects (taken from Naiita et al., 2003). The slope of the fitted line is a measure for the excitation temperature $T_{e}$ assuming LTE and optically thin gas.

Matter in the disk rotates approximately with Keplerian velocity $u_{\text {Kep }} \propto R^{-1 / 2}$ around the central star. If the disk is inclined with respect to the line of sight of the observer, the imprints of rotation can be seen in the line profiles. Material closer to the star rotates faster, hence, the contribution to the line originating there is broadened stronger than contribution from larger radii. Therefore, high-resolution spectroscopy is a good tool to retrieve spatial information about the line emitting regions. In the following the most important gas tracers are explained.

\subsubsection{CO emission}

The $\mathrm{CO}$ molecule has a high dissociation temperature of $\sim 4000-5000 \mathrm{~K}$ and will be in the gas phase down to $\sim 20 \mathrm{~K}$ where condensation on dust grains will occur. Two prominent CO bands have been observed in disks around young stars.

CO overtone emission lines arise from vibrational transition with $\Delta v=2$ and can be observed around $2.3 \mu \mathrm{m}$. CO overtone emission was one of the earliest probes for gas in disks around T Tauri stars (Scoville et al., 1983; Carr, 1989; Naiita et al., 1996). Najita et al. (2007) note that $\mathrm{CO}$ overtone emission lines are present in spectra of YSOs over a wide range of masses with a trend towards high-luminosity objects. The high excitation temperature $\sim 2000 \mathrm{~K}$ and densities $n>10^{10} \mathrm{~cm}^{-1}$ for overtone emission (due to small A-values) suggest an origin very close $(0.05<R<0.30 \mathrm{AU}$, e.g. Chandler et al., 1993) to the central star in CTTS disks with high accretion rates. Models for high mass stars suggest that the emission from these objects originates at radii $\sim 3 \mathrm{AU}$ (Blum et al., 2004; Bik \& Thi, 2004). Unfortunately, CO overtone emission is only observed in a small fraction of CTTS and HAeBe stars. Therefore, it is not well suited for the analysis of a brought range of YSOs.

The CO fundamental emission lines with $\Delta v=1$ are visible around $4.7 \mu \mathrm{m}$. They are detectable in most CTTS (Naiita et al., 2003) and many HAeBe stars (Blake \& Boogert, 2004) because the population of the $v=1$ energy level requires lower excitation temperatures and column densities than for the overtone $v=2$ level. Excitation temperatures are of the order of $1000 \mathrm{~K}$ and the lines are usually centrally peaked in contrast to the double-peaked profiles of the overtone lines (Carr, 2007). This can be readily explained by an extension of the line emitting region out to larger radii where the radial velocity shifts of the lines are smaller. Measurements of the full width half maximum (FWHM) of fundamental CO lines are typically in the range of $50-100 \mathrm{~km} \mathrm{~s}^{-1}$ which corresponds to an inner $\mathrm{CO}$ radius of $\lesssim 0.1 \mathrm{AU}$. The outer radius is proposed to be at $\gtrsim 1 \mathrm{AU}$ (Naiita et al., 2003), hence, CO fundamental emission originates from the terrestrial planet region. In high signal-to-noise observations, the $v=2-1$ transition can be observed and sometimes even the $v=1-0$ line of the ${ }^{13} \mathrm{CO}$ isotope. These lines depend 
strongly on temperature and densities, just as the overtone CO lines, and can, therefore, put tighter constraints on disk parameters.

CO emission line fluxes can be used to estimate excitation temperatures. Under the assumption of an optically thin gas and thermally populated energy levels, the logarithm of the quantity $X_{e}=F / \nu g_{u} A_{u l} \propto \exp \left(-E_{u} / k_{\mathrm{B}} T_{e}\right)$, where $F$ is the line flux, $g_{u}=2 J+1$ the statistical weight of the upper level, and $A_{u l}$ the Einstein A-value, is plotted against the energy $E_{u}$ of the upper level. Since $d\left(\ln X_{e}\right) / d E_{u}=-1 / k_{\mathrm{B}} T_{e}$, the slope of the curve gives the excitation temperature of the gas (see Fig. 1.10 Naiita et al., 2003). From this technique only a single temperature can be derived, however, the lines originate from different regions of different temperatures in the disk. Hence, the derived excitation temperature $T_{e}$ is an average value which is evident from the different slopes of grouped points around different $E_{u}$ values in Fig. 1.10.

Furthermore, $\mathrm{CO}$ observations show that the inner radius deduced from line width extends within the inner dust truncation radius. This not surprising because the dust destruction temperature of $\sim 1500 \mathrm{~K}$ is much lower than the $\gtrsim 4000 \mathrm{~K}$ needed for $\mathrm{CO}$ dissociation. More interestingly, the inner $\mathrm{CO}$ radii even tend to be of the order or even smaller than the corotation radii, the distance from the star where stellar angular velocity equals the local Kepler angular velocity. Therefore, the gaseous disk provides the material for high-energetic funnel flows (Shu et al., 1994) which originate at corotation radii.

\subsection{2 $\quad \mathrm{H}_{2}$ emission}

$\mathrm{H}_{2}$ is the most abundant molecule in interstellar material $\left(\left(\mathrm{H}_{2} / \mathrm{CO}\right)_{\mathrm{ISM}} \approx 10^{4}\right)$ and, hence, most likely also in circumstellar disks. However, the physical properties of molecular hydrogen make it hard to trace. It is a homonuclear molecule and therefore has no permanent dipole moment. The Einstein A-values describing the probability of spontaneous emission of the fundamental (quadrupole) transitions are small and give rise to weak lines. Furthermore, the lines are widely spaced in frequency and for each line an own observation with current spectrographs is necessary which makes the data collection very challenging.

$\mathrm{H}_{2}$ emission lines from disks can be observed in the near- and mid-IR when transitions between the rotational levels of the vibrational states 2 and 1 or 1 and 0 occur. The $\mathrm{H}_{2} v=2-1 S(1)$ line has first been detected in disks around the CTTS TW Hya (Weintraub et al., 2000) and the WTTS DoAr21 (Bary et al., 2002). Barv et al. (2003) report the observation of $\mathrm{H}_{2}$ in two more CTTS and measure line width of $\sim 10 \mathrm{~km} \mathrm{~s}^{-1}$ centered at the system velocity of the star, thus, being in agreement with an origin in the disk at distances $\gtrsim 10 \mathrm{AU}$. While temperatures of $\gtrsim 1000 \mathrm{~K}$ are necessary to excite the transitions thermally, these lines are most likely produced by non-thermal effects such as UV fluorescence or X-ray heating. It is difficult to measure the total gas mass from the $\mathrm{H}_{2}$ lines since the strength of emission is strongly depending on the intensity of the radiation field (large gas column density) or the gas mass (low column density).

The $\mathrm{H}_{2}$ lines arising from pure-rotational transitions with $\Delta J=2$ can be observed at mid-IR wavelengths, e.g. the $v=0-0(J=2-0) S(0)$ line at $28 \mu \mathrm{m}$, the $v=0-0 S(1)$ line at $17 \mu \mathrm{m}$, the $v=0-0 S(2)$ line at $12 \mu \mathrm{m}$, or the $v=0-0 S(3)$ line at $9.6 \mu \mathrm{m}$. They probe gas at $\sim 100 \mathrm{~K}$ which places their emission region in the giant planet formation zone. The small A-values allow detections up to large gas masses $\left(N_{\mathrm{H}} \gtrsim 10^{23} \mathrm{~cm}^{-2}\right.$ for the transition to become optically thick). If the continuum is already optically thick, then the $\mathrm{H}_{2}$ lines only probe a small fraction of the gas.

Electronic transitions of $\mathrm{H}_{2}$ can be observed in the ultraviolet (UV) wavelength range and hence only from space. Transitions between the first upper and the ground electronic states can 
be explained by Ly $\alpha$ emission pumping (e.g. Herczeg et al., 2004). All detected emissions that overlap with Ly $\alpha$ are pumped out of highly excited vibrational $v$ and in some cases also high rotational $J$ states. Consequently, assuming a thermal population of these lower states of the electronic transitions, the gas must have $\sim 1000 \mathrm{~K}$. The vast majority of accreting TTS shows $\mathrm{H}_{2}$ fluorescence (Naiita et al., 2007, and references therein) because Ly $\alpha$ emission is a typical signature of accreting $\mathrm{T}$ Tauri stars.

\subsection{3 $\quad \mathrm{H}_{2} \mathrm{O}, \mathrm{C}_{2} \mathrm{H}_{2}, \mathrm{HCN}$, and $\mathrm{OH}$ lines}

Water $\left(\mathrm{H}_{2} \mathrm{O}\right)$ is expected to be abundant in the gas phase of inner disks. Only recently, Carr et al. (2004) and Thi \& Bik (2005) reported the detection of hot water vapor in IR spectra of YSOs which also show $2.3 \mu \mathrm{m} \mathrm{CO}$ emission. Carr et al. (2004) modelled the $\mathrm{CO}$ and $\mathrm{H}_{2} \mathrm{O}$ lines and found that $\mathrm{H}_{2} \mathrm{O}$ originates at larger radii than the $\mathrm{CO}$ emission lines due to smaller velocity widths of the lines and lower excitation temperatures needed for the transitions. Additionally, they found a lower $\mathrm{H}_{2} \mathrm{O}$ abundance than expected from chemical equilibrium which they explain by strong incident irradiation.

$\mathrm{C}_{2} \mathrm{H}_{2}$ and HCN have been observed in absorption in IR spectra of TTS by Lahuis et al. (2006) and Gibb et al. (2007). The former authors derive excitation temperatures of $400-900 \mathrm{~K}$ and high abundances of $\mathrm{C}_{2} \mathrm{H}_{2}$ and $\mathrm{HCN}$ of $10^{-5}$ and $10^{-6}$ and estimate an origin at $R<1 \mathrm{AU}$ in the disk. An origin of the lines in a disk wind is considered by the authors.

Carr \& Naiita (2008) have used the IR space telescope Spitzer in order to detect $\mathrm{H}_{2} \mathrm{O}, \mathrm{OH}$, $\mathrm{HCN}, \mathrm{C}_{2} \mathrm{H}_{2}, \mathrm{CO}_{2}$, and $\mathrm{CO}$ in the CTTS AA Tau. Temperatures determined are around $500-$ $900 \mathrm{~K}$ which places the emission region inside $3 \mathrm{AU}$. The authors also find high abundances of the simple organics and $\mathrm{H}_{2} \mathrm{O}$ in comparison to hot molecular cores surrounding young protostars. Therefore, substantial molecular synthesis in the disk is suggested.

\subsection{Disk model codes}

A variety of model codes for the numerical simulation of the structure of (accretion) disks have been developed over the last decades. Different approaches have been used to tackle specific problems. Early models combined sets of blackbodies or stellar atmosphere models to simulate the excess flux observed in stellar systems with disks. Later, more detailed vertical disk models have been developed, however, treating the radiative transfer by the diffusion approximation which is only appropriate in optically thick media. Recent structure models are based on the standard accretion disk model (Shakura \& Sunvaev, 1973) and solve the structure and radiative transfer equation in the vertical direction for a number of disk rings. Also 2D (vertical and radial dimensions) Monte Carlo models for dust continuum radiative transfer simulations have emerged quite numerously. These usually do not solve the structure equations. Today, large computers allow to calculate radiative transfer in full 3D.

\subsubsection{Early models}

The pioneering work of Shakura \& Sunvaev (1973) and Lvnden-Bell \& Pringle (1974) laid the foundations for the description of accretion disks. The assumption of a geometrically thin disk (the height of the disk is much smaller than the radius) allows for the separate treatment of vertical and radial structure. The radial structure can be determined by adopting an axisymmetric and vertically averaged structure. Bath et al. (1980) used the description of Lvnden-Bell (1969) to 
estimate the flux of an accretion disk around a white dwarf in a cataclysmic variable (CV; binary system consisting of a compact white dwarf which accretes matter from a main sequence star that fills its Roche lobe) system by an integration of blackbody fluxes which originate from steadily accreting ( $\dot{M}=$ const.) and optically thick regions (annuli) of different effective temperatures. The approximate relation $F_{\nu} \propto \nu^{1 / 3}$, however, did not lead to satisfying fits. Adams et al. (1987a) use the same approximation in their effort to model the IR excess of flat and passive disks which reemit stellar irradiation.

An improvement over the blackbody models in the case of CV accretion disks was the use of stellar atmosphere models of effective temperature and surface gravity characteristic for a given disk annulus at radius $R$ (e.g. Kiplinger, 1979; Mavo et al., 1980). Both the blackbody and the stellar atmosphere approximation for accretion disks in CVs have been shown to be inappropriate (Wade, 1988).

For passive irradiated disks around young stars, an improvement in SED fits have been achieved by allowing the disks to flare as a consequence of vertical hydrostatic equilibrium (Kenvon \& Hartmann, 1987). In that case especially the outer regions of disks receive more stellar irradiation and the temperature increases. The disk flux calculated from blackbodies reproduce the observed SEDs better.

\subsubsection{Vertical structure models}

Further improvements in the understanding of disks has been achieved by treating the vertical structure of disks in more detail. Mever \& Mever-Hofmeister (1982) and Cannizzo et al. (1982) presented vertical steady accretion disk models including convection. Because they intended to investigate the stability of such disks in CVs, the radiative transfer was only calculated in the diffusion approximation which is valid only at large optical depth. Therefore, no emergent radiation field could be derived. A similar approach was chosen by D'Alessio et al. (1998) in the case of accretion disks around young stars. They calculate detailed hydrostatic models including irradiation by the central star, viscous dissipation, and convection but the radiative transfer was solved in a simplified manner using frequency-integrated moment equations and mean opacities. Chiang \& Goldreich (1997) calculated SEDs for CTTS with passive circumstellar disks assuming that an optically thin layer of superheated dust grains resides on a dense inner layer. In their model the surface is heated by stellar irradiation and about half of the stellar energy is reemitted into space while the other half heats the inner parts of the disk.

Kriz \& Hubenv (1986) and Shaviv \& Wehrse (1986) went beyond the simplified radiative transfer approaches and presented accretion disk models with frequency dependent radiation field treatment. Hubenv (1990) derived a simplified analytical model which provides equations for the temperature and density structure. Calvet et al. (1991) were the first to analyse the influence of irradiation on the temperature structure and the emergent spectrum (emission vs. absorption lines) for accretion disks around young stars using fequency-integrated quantities to describe the radiation field. Dullemond et al. (2002) showed vertical structure models for passive irradiated disks around $\mathrm{T}$ Tauri and Herbig Ae/Be stars treating only the dust in the disk ignoring scattering. They compare vertical temperature structures obtained from full frequency-dependent radiative transfer and frequency-averaged moment equations and show that a detailed treatment of the radiation field is crucial.

Nagel et al. (2004) developed a code that solves the structure equations with detailed radiative transfer iteratively using gas opacities and non-LTE model atoms necessary for the modellig of hot CV disks. In this work vertical structure models of relatively cool accretion disks around YSOs 
will be presented (see also Hügelmever et al., 2009). Gas and dust opacities are included and their abundances are calculated assuming chemical equilibrium. The irradiation from the central star is treated in detail and the radiative transfer equation is solved including scattering using the operator splitting method (Hauschildt \& Baron, 1999). Hydrostatic and radiative equilibirum are considered and the set of coupled structure equations is solved self-consistently.

\subsubsection{Multi-D codes}

In the case of disks around young stars, the irradiation of the central star onto its surrounding material is very important for the modelling of the radiation field. The dust in the disk is less abundant than the gas but dominates the opacity. Assuming that the disk is axisymmetric, solving the RT equation in 2D spatial configurations is more realistic in a geometrical sense than the vertical structure models described above. Since the computational realisation is more challenging (e.g. memory handling) in the 2D than in the 1D case, certain simplifications have to be applied. There are a number of $2 \mathrm{D} / 3 \mathrm{D}$ dust continuum radiative transfer codes either using a Monte Carlo solver for the RT problem (e.g. Wolf et al., 1999; Dullemond \& Dominik, 2004) or explicit approaches (e.g. Dullemond \& Turolla, 2000; Steinacker et al., 2003). These codes are suited for the calculation of dust temperature structures, intensity maps, and SEDs. In the realisation of the Monte Carlo code by Dullemond \& Dominik (2004), photon packages emitted by the central star are followed through the grid and absorption and re-emission processes determine the temperature under the assumption of temperature independent opacities. A benchmark test for different codes is presented by Pascucci et al. (2004), where the density and geometrical structure is given and not iterated. Woitke et al. (2009) developed a 2D radiation thermo-chemical model. They solve the chemistry for 71 species taking into account 950 reactions and combine the results with wavelength-dependent dust continuum RT and a consistent calculation of the hydrostatic structure.

The next step is to perform full 3D radiative transfer including gas and dust opacities as well as scattering. This way the abundant gas in the disk can be considered and non-axisymmetric effects can be simulated. In a series of papers (Hauschildt \& Baron, 2006; Baron \& Hauschildt, 2007; Hauschildt \& Baron, 2008, 2009; Baron et al., 2009) Hauschildt and Baron describe a framework for such 3D radiative transfer simulations in different coordinate systems and for various simulation goals, e.g. grey RT or two-level model atom line transfer. In this thesis the framework has been adopted to take 1D disk structures as input and interpolate those on a 3D Cartesian or cylindrical grid. Besides grey radiative transfer, a wavelength dependent module has been developed which takes binned opacities from 1D calculations. Furthermore, a line RT as described in Baron \& Hauschildt (2007) has been adopted for the use with differentially rotating disks.

\subsection{Infrared spectroscopy}

YSOs are characterised by their IR flux excess which originates in the circumstellar disk with typical temperatures of $\sim 10-1500 \mathrm{~K}$. In order to investigate the properties of protoplanetary disk a comparison of synthetic disk spectra with observed high-resolution IR spectra is a good choice. In this Section the properties of the ESO VLT spectrograph CRIRES will be presented and the data reduction will be explained.

CRIRES is the CRyogenic high-resolution InfraRed Echelle Spectrograph (Kaeufl et al., 2004) which is mounted to the Nasmyth focus A of UT1. A maximal resolving power of $R \sim 100000$ in the near- to mid-IR wavelength range of $0.95-5.20 \mu \mathrm{m}$ can be achieved using a slit width of 0.2 . 

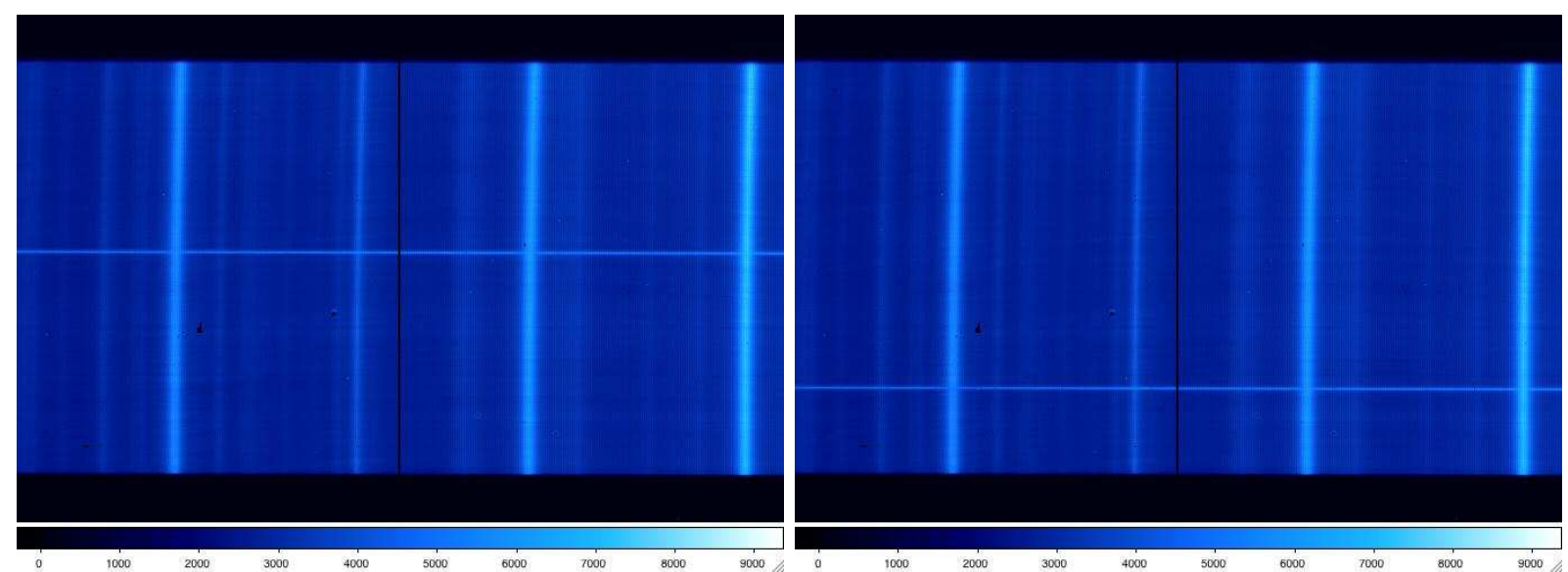

Figure 1.11: CRIRES images of a standard star with telluric emission lines superimposed for two different nodding positions of the object in the slit.

The detector is a mosaic of four Aladdin III InSb arrays providing an overall number of $4096 \times 512$ pixels. The gap between the individual chips is equivalent to 283 pixels. Therefore, breaks in the spectral coverage are present but they can be closed by taking a second spectrum shifted in wavelength which then also covers the gap. The spectrograph is of Echelle type but it is not crossdispersed, hence, only one order can be detected at a time. Since telluric absorption and emission lines (intrinsic to the Earth's atmosphere) contaminate the spectrum of the observed object, these unwanted features have to be eliminated in the data reduction process. The cancellation of telluric emission lines can be achieved rather easily by exposing the object on two different nodding positions A and B, i.e. shifting the object in the slit in spatial direction (see Fig. 1.11). The telluric emission lines can then be excluded from each image by subtracting the two images from each other $(\mathrm{A}-\mathrm{B}$ and $\mathrm{B}-\mathrm{A})$. During this process, the images are also divided by a normalised flat field image in order to reduce instrument or detector intrinsic curvatures in the intensities. In a next step, the 1D spectra are extracted from the images using the CRIRES reduction pipeline. Then the spectra with different nodding positions are combined in order to improve the signal-to-noise ratio.

This procedure is done for science as well as standard reference star images. The reference stars are chosen to be free of spectral emission or absorption features but ideally share the same telluric absorption lines as the science target. This is, however, only the case if the observation conditions for science and standard object are comparable, i.e. if the airmass and weather do not deviate much. Alternatively, telluric models can be calculated and fit to the absorption lines in the science spectrum. In order to eliminate the telluric absorption features, the standard star spectrum or the telluric model are scaled to the continuum of the science spectrum and the latter is divided by the first (see Fig. 1.12). 

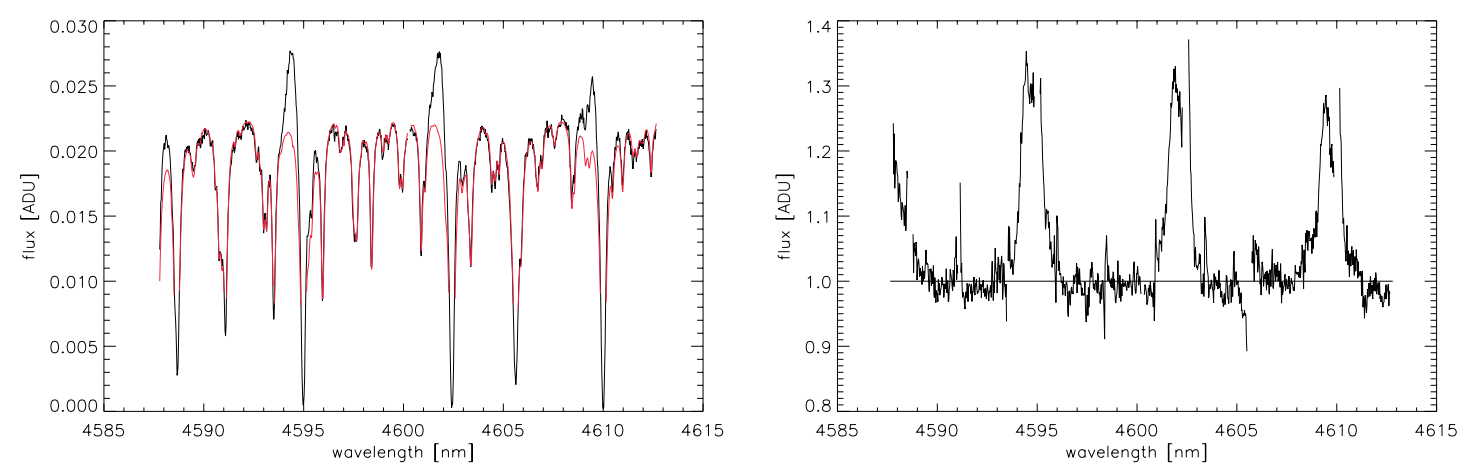

Figure 1.12: Left: Science (black line) and standard star (red line) spectra. The telluric absorption lines overlap. Right: Science spectrum normalised by division through the standard star. 


\section{Chapter 2}

\section{$1+1 \mathrm{D}$ models}

Accretion disks are three dimensional objects and a correct numerical treatment requires the solution of the structure and radiative transfer equations in all spatial directions. However, such an attempt is currently beyond computational capabilities if the radiative transfer is supposed to be treated in a sophisticated manner. Therefore, simplifications have to be applied. The approach followed in this work aims at modelling high-resolution spectra, hence, in order to allow calculations on reasonable time scales, the structure equations are limited to one dimension. Since accretion disks are radially extended objects, the total disk structure can be approximated by a set of disk annuli. The vertical structure is then assumed to be constant over ring width (see Fig. (2.1) and the combination of the calculated structures yields the total disk model.

The disk model implemented as part of this thesis is an extension to the general purpose stellar atmosphere code PHOENIX. The solution approach used in PHOENIX is a nested scheme iteration of the highly coupled structure equations. The iteration scheme is shown in Fig. 2.2. The solution of the equation of hydrostatic equilibrium, the boundary conditions of the radiative transfer equation, and the equation of radiative equilibrium had to be adapted for the properties of circumstellar disks. In this chapter, the basic ideas and equations for the solution of the disk atmosphere problem will be explained.

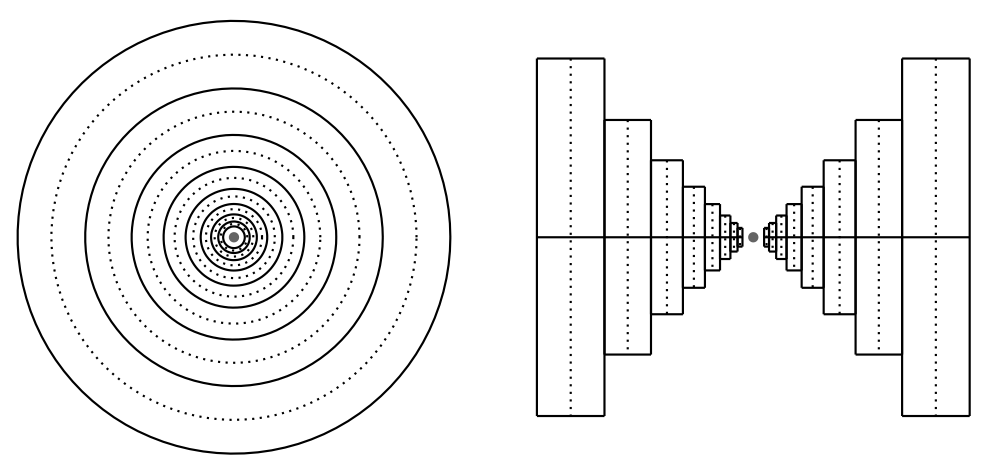

Figure 2.1: Disk ring geometry as adopted for the structure calculations. The radius of the rings increases exponentially. The left panel shows a face-on view of a disk, the right one a vertical cut viewed edge-on (height is not to scale). The dotted lines are the radii $R$ for which the models are calculated while the solid lines show the borders of a disk ring. The disk structure is assumed to be constant over the ring width. 


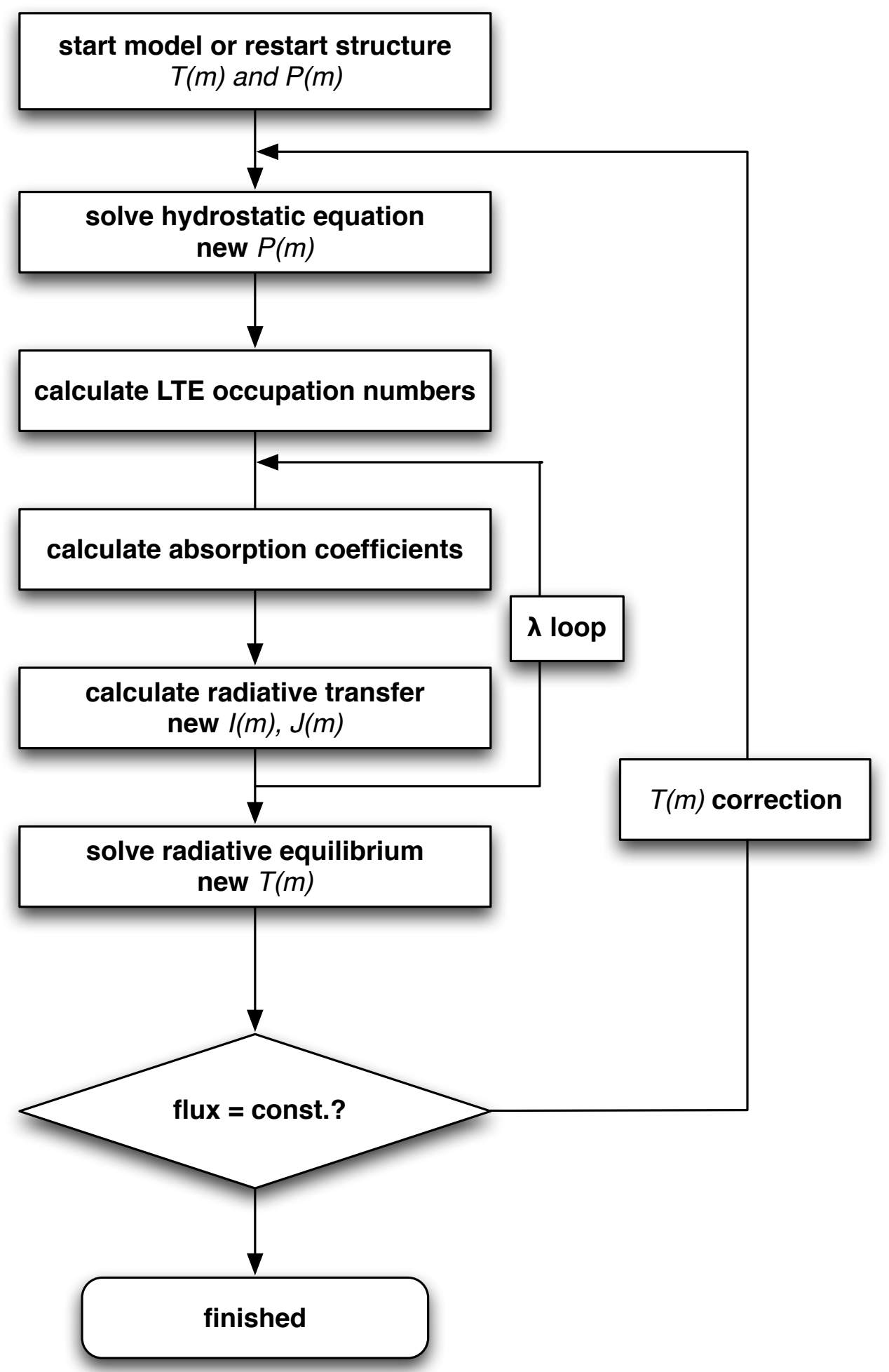

Figure 2.2: Flow chart showing the iteration scheme as used for the DISK model calculation with PHOENIX. 


\subsection{Standard accretion disk model}

The canonical model of Lynden-Bell \& Pringle (1974) and Shakura \& Sunvaev (1973) describes the radial structure of accretion disks. The disk is assumed to be cylindrically symmetric and geometrically thin, i.e. the disk height $H$ is much smaller than the disk ring radius $R$. In this description the vertical disk structure is in quasi-hydrostatic equilibrium compared to the time scales of radial motion of matter. Therefore, the vertical and radial structure can be described independently.

Matter is assumed to rotate with Keplerian velocities and viscous shear decelerates inner and accelerates outer parts. This leads to accretion of a large fraction of disk matter onto the star and outward transportation of angular momentum by a small fraction of disk matter. Molecular viscosity is too small to provide the observed mass accretion rates. Thermal convection in accretion disks was investigated by different authors using various methods which are summarised in Klahr (2007). The results show that thermal convection is unlikely the dominant source of turbulence and even leads to inward transport of angular momentum. Furthermore, a heating source is required to drive the convection. Bell \& Lin (1994) argue that convective instabilities can only occur at temperatures $T<200 \mathrm{~K}$ or $2000 \mathrm{~K}<T<20000 \mathrm{~K}$, i.e. temperature regimes untypical for inner protoplanetary disks. The magnetorotational instability (MRI) introduced by poloidal magnetic fields in ionised disk matter (Fricke, 1969; Balbus \& Hawlev, 1991), the global baroclinic instability caused by inclined density and pressure gradients in the disk (e.g. Klahr \& Bodenheimer, 2003), and stratification instabilities caused by a dust sub-disk (e.g. Johansen et al., 2006) are among the currently debated origins of dissipation and angular momentum transport, yet, their effect on the thermal structure of the disk cannot be easily described or parametrised. Even though temperatures $T>1000 \mathrm{~K}$ are necessary to thermally ionise disk material, cosmic ray ionisation is possible at surface densities $\Sigma<100 \mathrm{~g} / \mathrm{cm}^{2}$ (Gammie, 1996). The mean viscous dissipation is often modelled as an "alpha-viscosity" resulting in a vertically-averaged viscosity

$$
\bar{\nu}=\alpha c_{\mathrm{s}} H
$$

(Shakura \& Sunvaev, 1973) where $0 \leq \alpha \leq 1$ is the angular momentum transfer efficiency, $c_{\mathrm{s}}$ the sound speed, and $H$ the pressure scale height. Alternatively, a turbulent viscosity can be modelled assuming a critical Reynolds number Re

$$
\bar{\nu}=\frac{\sqrt{G M_{\star} R}}{R e},
$$

(Lvnden-Bell \& Pringle, 1974). This second model has the advantage, that the calculation of the mean viscous dissipation is decoupled from the thermal structure of the disk and is adopted in this work. Both of these formalisms allow one to account for the effect of viscosity on the disk structure without the need to describe its origin in detail.

The standard accretion disk model further allows to derive the following radial quantities. The mass surface density

$$
\Sigma=\int_{-\infty}^{+\infty} \varrho(z) d z
$$

where $\varrho$ is the density, can in the static case (constant mass accretion rate) be written as function of the radius $R$ as

$$
\Sigma(R)=\frac{\dot{M}}{3 \pi \bar{\nu}}\left[1-\left(\frac{R_{\star}}{R}\right)^{\frac{1}{2}}\right],
$$


where $\dot{M}$ is the mass accretion rate (Kriz \& Hubenv, 1986). Using Eq. (2.4) and assuming that disk matter moves at Keplerian angular velocity

$$
\Omega_{\mathrm{K}}=\sqrt{\frac{G M_{\star}}{R^{3}}}
$$

the dissipated energy rate per unit surface can be expressed by

$$
D(R)=\frac{3 G M_{\star} \dot{M}}{8 \pi R^{3}}\left[1-\left(\frac{R_{\star}}{R}\right)^{\frac{1}{2}}\right] .
$$

Equation (2.6) shows that the dissipated energy is independent of the viscosity $\nu$. With the simplification that the energy radiated from the disk can be described by the Stefan-Boltzmannlaw

$$
D(R)=\sigma T_{\mathrm{eff}}^{4}
$$

with $\sigma$ being the Stefan-Boltzmann constant, the effective temperature of the disk at a given radius can be written as

$$
T_{\mathrm{eff}}^{4}(R)=\frac{3 G M_{\star} \dot{M}}{8 \pi \sigma R^{3}}\left[1-\left(\frac{R_{\star}}{R}\right)^{\frac{1}{2}}\right],
$$

which is also independent of the viscosity $\nu$.

As shown above, the disk structure can be described in the picture of the standard accretion disk model by the mass and radius of the central object, the mass accretion rate, and the effective viscosity $\nu$.

\subsection{Radiation field}

The radiation field can be described by the specific intensity

$$
I_{\nu}(\vec{r}, \vec{n}, \nu, t)=\frac{d E_{\nu}}{d t d \nu d \omega \cos \vartheta d A},
$$

which is the energy $d E_{\nu}$ per time interval $[t, t+d t]$, frequency interval $[\nu, \nu+d \nu]$, solid angle $d \omega$, and projected area element $\cos \theta d A$, where $\theta$ is the angle under which the radiation leaves the surface of the radiating matter.

Integration of the weighted specific intensity $I_{\nu} \mu^{i}(\mu:=\cos \theta)$ over all directions $\vec{n}$ defines the $i$ th moment of the radiation field. Hence, the zero-order moment or mean intensity is calculated as

$$
J_{\nu}(\vec{r}, \nu)=\frac{1}{4 \pi} \int_{4 \pi} I_{\nu}(\vec{r}, \vec{n}, \nu) d \omega
$$

where the time-dependence was neglected. For a plane-parallel one-dimensional treatment of the radiation field Eq. (2.10) can be written as

$$
J_{\nu}(z, \nu)=\frac{1}{2} \int_{-1}^{1} I_{\nu}(z, \mu, \nu) d \mu
$$




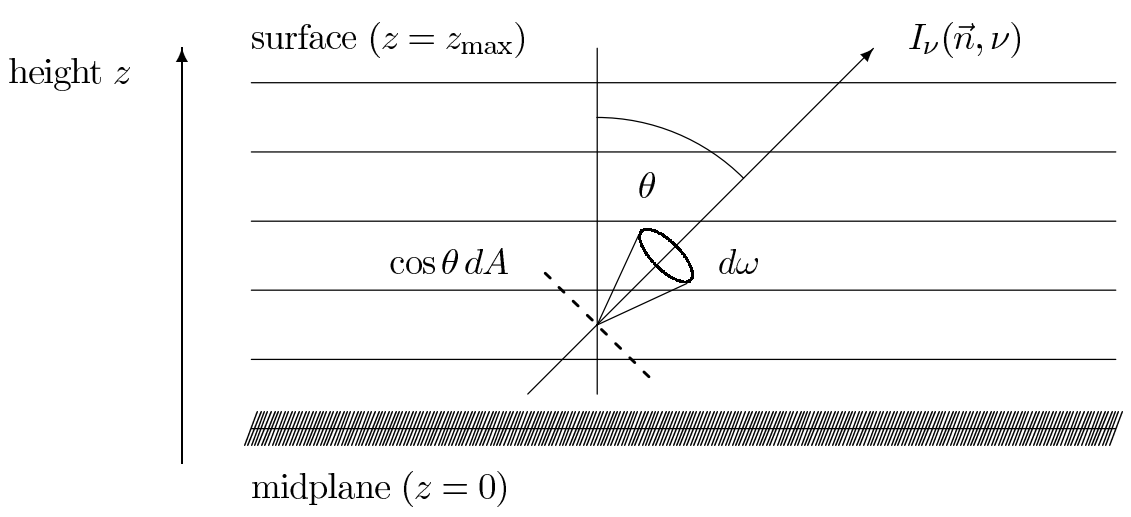

Figure 2.3: Schematic sketch of a plane parallel disk atmosphere. The vector tilted by the angle $\theta$ against the normal to the plane is the ray along which the intensity $I_{\nu}(\vec{n}, \nu)$ is calculated.

The first- and second-order moment can be written accordingly:

$$
\begin{aligned}
& H_{\nu}(z, \nu)=\frac{1}{2} \int_{-1}^{1} I_{\nu}(z, \mu, \nu) \mu d \mu \\
& K_{\nu}(z, \nu)=\frac{1}{2} \int_{-1}^{1} I_{\nu}(z, \mu, \nu) \mu^{2} d \mu
\end{aligned}
$$

\subsection{Gas and dust properties}

\subsubsection{Occupation numbers}

Disk atmospheres are open systems and radiation can escape transporting away the energy produced by dissipation or due to irradiation by the central star. In atmospheric regions where collision rates between particles are high and exceed photon-particle interactions, however, it is a good approximation to assume local thermodynamic equilibrium (LTE). In LTE systems the ratio of the occupation numbers of energy levels $i$ and $j$ of a species can be described entirely by the Boltzmann equation

$$
\frac{n_{i}}{n_{j}}=\frac{g_{i}}{g_{j}} \exp \left(-\frac{E_{i j}}{k_{\mathrm{B}} T}\right),
$$

where $g$ is the statistical weight of a given atomic state and $E_{i j}$ is the energy needed for a transition between level $i$ and $j$. For atoms, $i \rightarrow j$ is an electronic transition where as for molecules the transition can be between electronic, rotational, vibrational, or translational states. If departures from LTE have to be considered, the occupation numbers need to be determined by iteratively solving a rate equation system. For all calculations presented in this thesis LTE is assumed because non-LTE model molecules are not available and would require enormous amounts of computational power. Non-LTE for atoms is neglected in the model calculations shown here because atomic spectral lines are scarce in IR observations of protoplanetary disks and their analysis is not the objective of this work. 


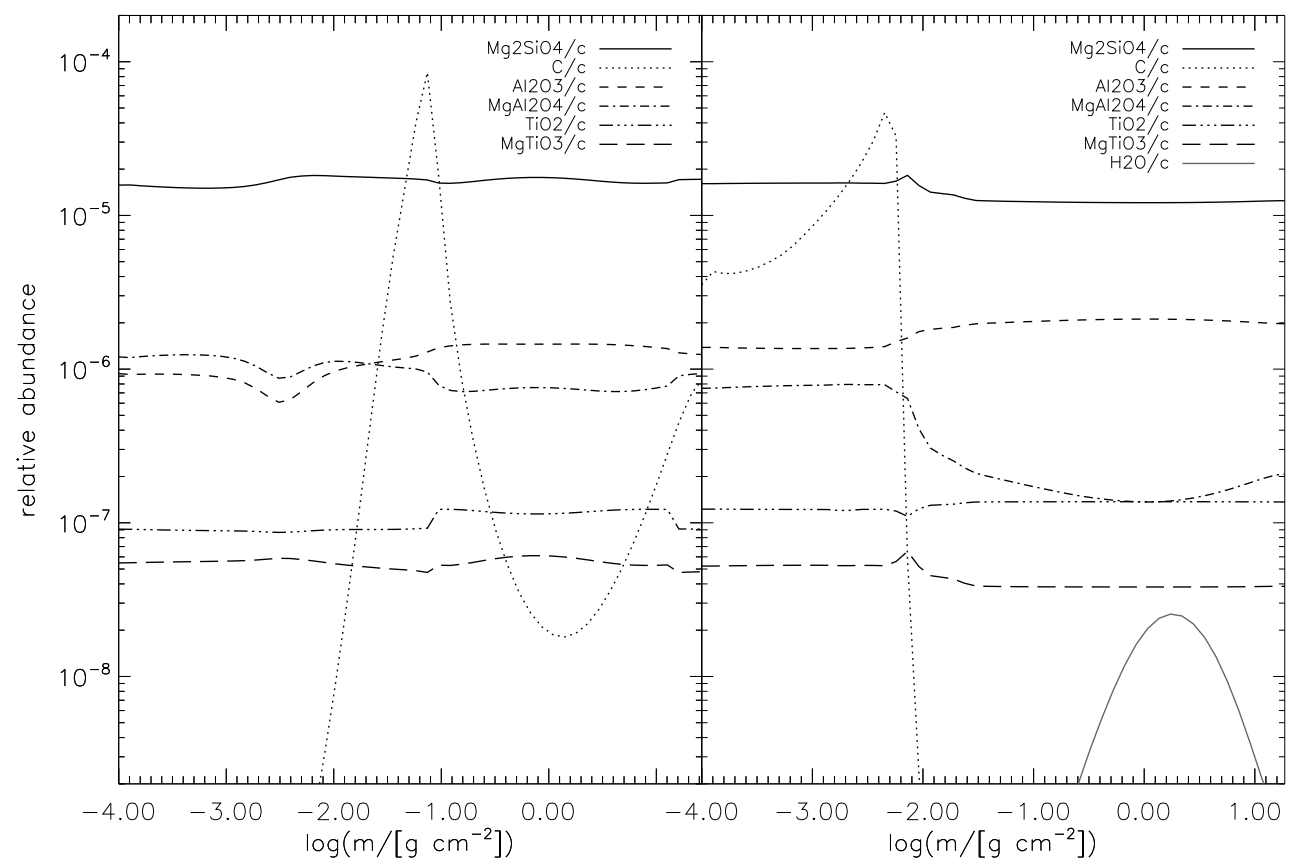

Figure 2.4: Relative abundances of the most important dust contributors to the opacity. The left plot is for a disk ring with $R=0.094 \mathrm{AU}$ and the right one for $R=0.290 \mathrm{AU}$. Forsterite $\left(\mathrm{Mg}_{2} \mathrm{SiO}_{4}\right)$ is the most abundant dust species and strongly present in all layers. Graphite sets on around $m=0.01 \mathrm{~g} / \mathrm{cm}^{2}$ where it blocks stellar irradiation efficiently and therefore suppresses heating of deeper layers.

\subsubsection{Dust chemistry and opacity}

Dust is included in the models presented here by treating condensate formation under the assumption of chemical equilibrium and phase-equilibrium for 217 dust species. I.e. all dust monomers exceeding the local saturation pressure as defined by thermal equilibrium are allowed to condense (this implies a supersaturation ratio $S=1$; Allard et al., 2001, cf. also Helling et al. 2008 for a comparison of different condensation treatments).

The grain opacity is calculated from the most important refractory condensates using optical data for a total of 50 different species. Absorption and scattering are calculated in the Mie formalism, assuming a given particle size distribution for a mixture of pure spherical grains, following the general setup of the new ACES dust setup (Barman, private communication; a plot with relative abundances of the most important species in the disk models is shown in Fig. 2.4). This equilibrium assumption is a good approximation in the inner optically thick layers of the well-mixed disk atmosphere. In the low-density outer layers, non-equilibrium effects are more important; they are however not included in the current version of the DISK code. In the later phases of disk evolution grain growth will become important and may cause departures from a homogeneous size distribution.

\subsection{Start models}

In order to start a new DISK disk calculation either an existing model can be used as input or a grey LTE start model is constructed. In the latter case, the approach of Hubenv (1990) is 
followed.

The model is initialised by setting up a mass depth scale $m_{i}, i=1, \ldots, \mathrm{NL}$, where NL is the number of layers, which will be kept fixed also for later calculations with the same input parameters. The $m$ scale is equally spaced on a logarithmic grid between the column mass at the midplane of the disk

$$
M_{0}=\frac{\dot{M}}{6 \pi \bar{\nu}}\left[1-\left(\frac{R_{\star}}{R}\right)^{\frac{1}{2}}\right]
$$

and the outer value $m_{1}$, which is an input parameter. This spacing is done for $\mathrm{NL}-\mathrm{N}_{\text {in }}$ points, while for the remaining $N_{\text {in }}$ layers each point $j, j=1, \ldots, \mathrm{N}_{\mathrm{in}}$, is positioned halfway between $M_{0}$ and $m_{j-1}$, with $m_{j=0}=m_{\mathrm{NL}-\mathrm{N}_{\mathrm{in}}-1}$. This is done to provide numerical stability in the iterative process because of the steep $m$ gradient near the midplane. A typical value for $\mathrm{N}_{\text {in }}$ is 6 or 12 for $\mathrm{NL}=64$.

In a next step, the depth-dependent viscosity is calculated

$$
\nu(m)=\bar{\nu}(\zeta+1)\left(\frac{m}{M_{0}}\right)^{\zeta}
$$

assuming a value of $\bar{\nu}$ determined by an assumed constant critical Reynolds number (Eq. (2.2) ), where $\zeta>0$ is a free parameter (Kriz \& Hubenv, 1986). The smaller the value of $\zeta$, the lower the temperature. However, if irradiation is noticeably strong, the effect of $\zeta$ on the structure has negligible influence on the spectrum. Lvnden-Bell \& Pringle (1974) argue that the Reynolds number has to be chosen to equal the critical one for the onset of turbulence.

Furthermore, an isothermal density structure - the sound speed associated with the gas pressure $c_{\mathrm{g}}$ and the flux mean opacity $\kappa_{H}$ are independent of height - is assumed, which lets one derive a simple analytic expression for the density at each depth point $\varrho(z)$. The relation

$$
m(z)=\int_{z}^{\infty} \varrho(z) d z
$$

is then inverted to convert the mass depth variable $m$ into a height above the midplane of the disk $z$.

Finally, the formal LTE solution derived in Hubenv (1990) and the Rosseland opacity tables of Ferguson et al. (2005) are used to get a temperature structure by iterating

$$
T^{4}=\frac{3}{4} T_{\text {eff }}^{4}\left[\tau\left(1-\frac{\tau}{2 \tau_{\text {tot }}}\right)+\frac{1}{\sqrt{3}}+\frac{1}{3 \epsilon \tau_{\text {tot }}} \frac{\nu}{\bar{\nu}}\right]
$$

with $\kappa_{R}=\kappa_{R}(T, \varrho)$ until the $T$ - $\tau$-structure is consistent for each layer. In Eq. (2.18) $\epsilon=\kappa_{R} / \kappa_{B}$ is set equal to 1 and $\tau_{\text {tot }}$ is the opacity at the midplane of the disk.

The start models in the form implemented do not include irradiation by the central star. Furthermore, detailed RT calculations with PHOENIX lead to temperature changes of up to a few $100 \mathrm{~K}$. Therefore, a few detailed RT and structure iterations have to be made before irradiation is switched on in order to achieve stability in the temperature correction scheme.

\subsection{Iterative procedure}

After a restart model is read in or a start structure has been computed, the hydrostatic equation, the radiative transfer ( $\mathrm{RT}$ ) equation, and the energy balance equation have to be solved. This is 
done iteratively until convergence in flux is obtained. The termination criterion is

$$
\max _{i=1, \ldots, \mathrm{NL}}\left|\frac{F_{\mathrm{m}}\left(m_{i}\right)-F_{\mathrm{c}}\left(m_{i}\right)}{F_{\mathrm{m}}\left(m_{i}\right)}\right|<10^{-3}
$$

where $F_{\mathrm{m}}$ is the expected "mechanical" flux released by the disk and $F_{\mathrm{c}}$ the current flux value. This convergence criterion in Eq. (2.19) is not always reached and for a sufficiently small

$$
\max _{i=1, \ldots, \mathrm{NL}}\left|\Delta T_{i}\right| \ll 1 \mathrm{~K}
$$

the calculation is then stopped after a given number of iterations. Typical errors in $F$ are of the order of $10^{-2}$.

\subsection{Hydrostatic equilibrium}

The equation of hydrostatic equilibrium has to be satisfied for a disk atmosphere to be in a stable configuration. The mass of the disk is much smaller than that of the central star and self-gravitation of the disk can be neglected if $g_{\text {self }} \ll g_{\star}$, which is the case for the inner disks of low-mass stars analysed in this thesis. Assuming that the radial component of the gravitation of the central star and the centrifugal forces of the rotating disk just cancel each other out, the vertical hydrostatic equation reads

$$
\frac{d P}{d m}=\frac{G M_{\star}}{R^{3}} z
$$

In equation $2.21 P$ is the sum of the gas pressure

$$
P_{\mathrm{g}}=n k_{\mathrm{B}} T,
$$

where $n$ is the total particle density, and the radiation pressure

$$
P_{\mathrm{r}}=\frac{4 \pi}{c} \int_{0}^{\infty} K_{\nu} d \nu .
$$

Now, there are different ways to solve this equation. One constraint is the symmetry condition at the midplane of the disk. Therefore, $P^{\prime}\left(M_{0}\right)=0$ has to be satisfied. This boundary condition is naturally satisfied by the following approach:

By differentiating equation 2.21 over $m$ and applying the relation $d z / d m=-1 / \rho$, one obtains an expression for the hydrostatic equation which is independent of the height $z$ which still needs to be determined:

$$
\frac{d^{2} P}{d m^{2}}=-\frac{c_{\mathrm{s}}^{2}}{P} \frac{G M_{\star}}{R^{3}} .
$$

The sound speed $c_{\mathrm{s}}^{2}=P / \varrho$ is introduced, which is taken from the previous iteration and only depends on the temperature $T$. Therefore, the error in $P$ and $\varrho$ cancel out and one obtains a more stable version of Eq. (2.21). This second-order differential equation is then decomposed into two coupled first-order differential equations. With the inner boundary condition $P^{\prime}\left(M_{0}\right)=0$ (symmetry at the midplane) and the outer boundary condition

$$
P\left(m_{1}\right)=\frac{m_{1} c_{\mathrm{s}}^{2}}{H_{g} f\left(\frac{z-H_{r}}{H_{g}}\right)}
$$


derived in Hubenv (1990), the two point boundary value problem is solved with a simple shooting method.

Another solution ansatz is a simple integration from the top of the atmosphere to the midplane (Mihalas, 1978). In this approach one solves for the gas pressure $P_{\mathrm{g}}$ by rewriting Eq. (2.21):

$$
\frac{d P_{\mathrm{g}}}{d m}=\frac{G M_{\star}}{R^{3}} z-\frac{4 \pi}{c} \int_{0}^{\infty}\left(\chi_{\nu} / \varrho\right) H_{\nu} d \nu
$$

and discretising to

$$
P_{1}=m_{1}\left(\frac{G M_{\star}}{R^{3}} z_{1}-\frac{4 \pi}{c} \int_{0}^{\infty}\left(\chi_{\nu} / \varrho\right) H_{\nu} d \nu\right)
$$

for the outer starting value and

$$
P_{d}=P_{d-1}-\frac{4 \pi}{c}\left(K_{d}-K_{d-1}\right)+\frac{G M_{\star}}{R^{3}} z_{d} \cdot\left(m_{d}-m_{d-1}\right)
$$

with $K_{d}$ being the second frequency-integrated moment of the radiation field, for all other layers. This apparently more simple approach, however, leads to iterative oscillations in $P$ for disk rings with parameters typical for CTTS while the second order differential equation for $P$ given in Eq. (2.24) is very stable and quickly converges to a stable solution.

\subsection{Radiative transfer}

Each disk ring is assumed to be a plane-parallel slab of matter and the solution of the 1D radiative transfer equation (RTE)

$$
\mu \frac{\partial I_{\nu}(z, \mu, \nu)}{\partial z}=-\chi_{\nu}(z, \nu) I_{\nu}(z, \mu, \nu)+\eta_{\nu}(z, \nu)
$$

yields the intensity $I_{\nu}(z, \mu, \nu)$ in every layer of the disk ring between the midplane $z=0$ and the surface of the atmosphere $z_{\max }=z\left(m=M_{0}\right)$. In Eq. (2.29) the change in intensity $d I_{\nu}$ at a given frequency $\nu$ is equal to the sum of attenuation of $I$ given by $-\chi_{\nu} I_{\nu} d z$ and the contribution through emissivity $\eta_{\nu} d z$. In the special case of disk atmospheres, the inner and outer boundary conditions have to be set according to

$$
\begin{aligned}
& I_{\nu}\left(z_{\max },-\mu, \nu\right)=I_{\nu}^{\mathrm{ext}}\left(z_{\max },-\mu, \nu\right) \\
& I_{\nu}^{-}(z=0,-\mu, \nu)=I_{\nu}^{+}(z=0,+\mu, \nu)
\end{aligned}
$$

(the calculation of $I_{\nu}^{-}(z=0,-\mu, \nu)$ is described in Sect. 2.8). Using the definition of the source function $S_{\nu}=\eta_{\nu} / \chi_{\nu}$ one can rewrite Eq. (2.29)

$$
\mu \frac{d I_{\nu}}{d \tau_{\nu}}=I_{\nu}-S_{\nu}
$$

where $d \tau_{\nu}=\chi_{\nu} d z$ is the optical depth and $\mu$ and $z$ dependencies have been dropped for better readability. 
The source function $S_{\nu}$ in the radiative transfer equation (2.32) contains scattering terms which in turn depend on the radiation field. Therefore, the solution of the RTE has to be iterated because $S_{\nu}$ is not initially known. A formal solution of the RTE assuming that $S_{\nu}$ is known yields

$$
\begin{aligned}
& I_{\nu}^{+}\left(\tau_{\nu}\right)=I_{\nu}^{+}\left(\tau_{\max }\right) \cdot \exp \left(-\frac{\tau_{\max }-\tau_{\nu}}{\mu}\right)+\int_{\tau_{\nu}}^{\tau_{\max }} S_{\nu}\left(\tau^{\prime}\right) \cdot \exp \left(-\frac{\tau^{\prime}-\tau_{\nu}}{\mu}\right) \frac{d \tau^{\prime}}{\mu} \\
& I_{\nu}^{-}\left(\tau_{\nu}\right)=I_{\nu}^{-}\left(\tau_{\nu}=0\right) \cdot \exp \left(-\frac{\tau_{\nu}}{|\mu|}\right)+\int_{0}^{\tau_{\nu}} S_{\nu}\left(\tau^{\prime}\right) \cdot \exp \left(-\frac{\tau_{\nu}-\tau^{\prime}}{|\mu|}\right) \frac{d \tau^{\prime}}{|\mu|}
\end{aligned}
$$

The mean intensity can be written as

$$
\begin{aligned}
J_{\nu}\left(\tau_{\nu}\right) & =\frac{1}{2} \int_{-1}^{+1} I_{\nu}\left(\tau_{\nu}\right) d \mu \\
& =\frac{1}{2}\left(\int_{0}^{1} d \mu \int_{\tau_{\nu}}^{\infty} S_{\nu}\left(\tau^{\prime}\right) \cdot \exp \left(-\frac{\tau^{\prime}-\tau_{\nu}}{\mu}\right) \frac{d \tau^{\prime}}{\mu}\right. \\
& \left.+\int_{-1}^{0} d \mu \int_{0}^{\tau_{\nu}} S_{\nu}\left(\tau^{\prime}\right) \cdot \exp \left(-\frac{\tau_{\nu}-\tau^{\prime}}{(-\mu)}\right) \frac{d \tau^{\prime}}{(-\mu)}\right)
\end{aligned}
$$

assuming $\tau_{\max }=\infty$. This equation can be rewritten as

$$
J_{\nu}\left(\tau_{\nu}\right)=\frac{1}{2} \int_{0}^{\infty} S_{\nu}\left(\tau^{\prime}\right) E_{1}\left(\left|\tau^{\prime}-\tau_{\nu}\right|\right) d \tau^{\prime}
$$

with the exponential integral $E_{1}(x)=\int_{1}^{\infty} \exp (-x t) d t / t$. With the introduction of the $\Lambda$-operator

$$
\Lambda_{\tau}[f(t)] \equiv \frac{1}{2} \int_{0}^{\infty} f(t) E_{1}(|t-\tau|) d t
$$

the formal solution reads

$$
J_{\nu}\left(\tau_{\nu}\right)=\Lambda_{\tau_{\nu}}\left[S_{\nu}(\tau)\right]
$$

It is obvious from Eq. (2.40) that $J_{\nu}\left(\tau_{\nu}\right)$ is a non-local quantity since it depends on the source function $S_{\nu}$ at all optical depths $\tau$. In order to obtain the mean radiation field, the iterative scheme

$$
J_{\text {new }}=\Lambda\left[S_{\text {old }}\right] \quad \Leftrightarrow \quad S_{\text {new }}=(1-\epsilon) J_{\text {new }}+\epsilon B \quad,
$$

where $\epsilon$ is the thermal coupling parameter, has to be solved. In order to solve Eq. (2.41) numerically, it is handy to consider $\Lambda$ in its matrix representation. Then the mean intensity at a certain depth $i$ can be written as

$$
J_{i}=\sum_{j=1}^{\mathrm{NL}} \Lambda_{i j} S_{j} .
$$

The solution of Eq. (2.41) is called lambda iteration and suffers from severe convergence problems if $\epsilon \ll 1$ since the maximum eigenvalue of the amplification matrix is close to unity. Therefore the change per iteration can be small even though the current value of $J$ is far from the solution 


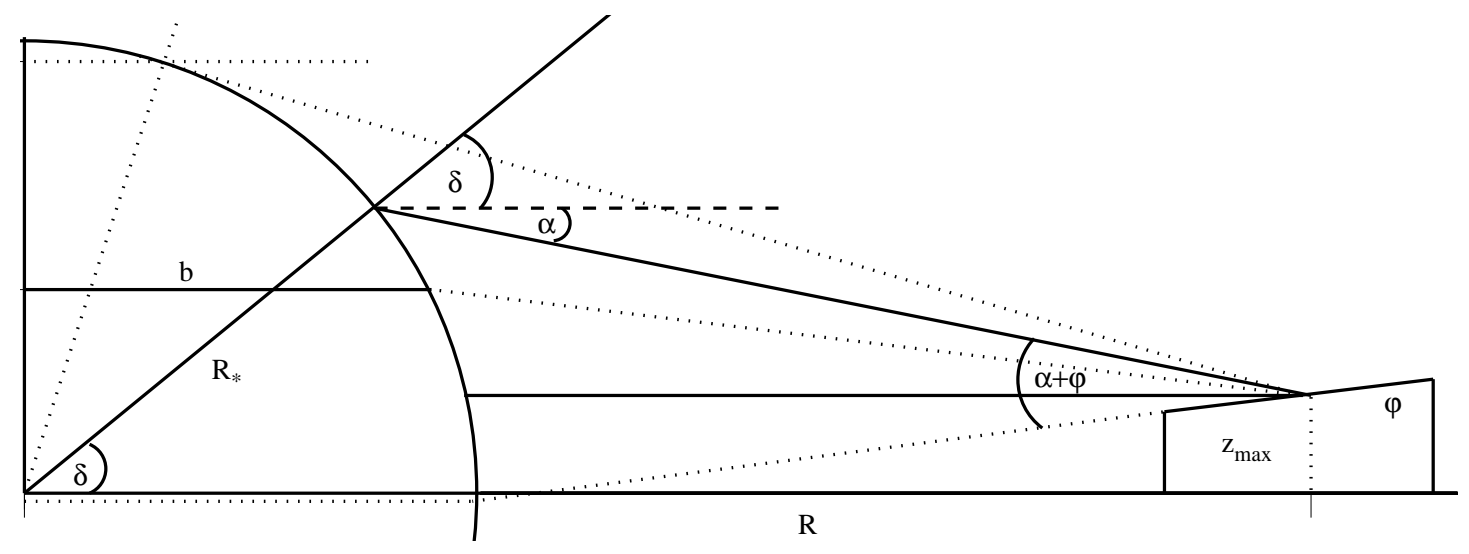

Figure 2.5: Irradiation geometry as adopted for the model calculations. A star with radius $R_{\star}$ at a distance $R$ from a disk ring with a height $z_{\max }$ and a slope of the disk surface $\tan (\varphi)$ is considered. For each quadrature point $\mu_{i}=\cos (\alpha+\varphi+\pi)$ and its corresponding integration weight $a_{i}$ the projected surface fraction on the star is determined and the irradiation intensity is calculated. The angle under which radiation leaves the surface of the star is $\mu_{j}=\cos (\alpha+\delta)$.

(Mihalas, 1978). This problem is overcome by operator splitting and introducing an approximate lambda operator $\Lambda^{\star}$ and split $\Lambda$ according to

$$
\Lambda=\Lambda^{\star}+\left(\Lambda-\Lambda^{\star}\right)
$$

A common and mostly adequate choice for the $\Lambda^{\star}$-operator is simply the diagonal (local) or the tri-diagonal (non-local) of the $\Lambda$-matrix. Other bandwidths can be chosen if the convergence properties require it. The elements of the $\Lambda$ and hence the $\Lambda^{\star}$-operator are calculated using the characteristic method (Olson \& Kunasz, 1987); Hauschildt et al., 1994). The symmetry boundary condition at the midplane (see Eq. (2.31)) had to be integrated into the $\Lambda^{\star}$ as well.

With the help of the operator splitting (Eq. (2.43) ) the solution for the mean intensity now reads

$$
J_{\text {new }}=\Lambda^{\star} S_{\text {new }}+\left(\Lambda-\Lambda^{\star}\right) S_{\text {old }}
$$

or

$$
\left[1-\Lambda^{\star}(1-\epsilon)\right] J_{\text {new }}=J_{\mathrm{fs}}-\Lambda^{\star}(1-\epsilon) J_{\text {old }}
$$

with $J_{\mathrm{fs}}=\Lambda S_{\text {old }}$. The solution for a local $\Lambda^{\star}$-operator is simply a scalar division. For a non-local operator a matrix inversion has to be performed which is numerically more costly but speeds up the convergence process.

\subsection{Irradiation geometry}

The treatment of irradiation of the central star upon the disk atmosphere is very important for spectral line diagnostics of protoplanetary disks. As shown by Calvet et al. (1991), the ratio between disk surface temperature, which is a function of the radius $R$ of the disk annulus and the effective temperature of the central star, and the intrinsic temperature caused by viscous dissipation determines whether spectral lines, e.g. of CO, appear in absorption or emission. Therefore, 
it matters significantly whether the whole star is assumed to irradiate the inner parts of the disk or only a fraction of the star is irradiating the disk due to self-shadowing.

The approach that is implemented in the 1D model code presented here is a geometrical one taking into account stellar limb darkening, self-shadowing by the disk, and the slope of the disk atmospheric height $z_{\max }(R)$. Figure 2.5 shows a plot with the irradiation geometry.

At the beginning of every model run with the irradiation option switched on, the slope of the disk atmosphere surface $\tan (\varphi)=\Delta z_{\max } / \Delta R$ at ring radius $R$ is determined by calculating the height of the disk according to the start model (see Sect. 2.4) at $R^{\prime}=R \pm \Delta R$ with $\Delta R=R \times 10^{-2}$. For each quadrature point $\mu_{i}$ (denoted $\alpha+\varphi$ in Fig 2.5) and corresponding integration weight $a_{i}$ the projected surface fraction of the star $A_{i}^{\text {proj }}$ is determined. This is done by first determining the maximum incident angle $\mu_{\max } \leq 0$ on the disk

$$
\mu_{\max }=\cos \left(\arctan \left(\frac{R_{\star}-z_{\max }}{R}\right)+\arctan \left(\frac{\Delta z_{\max }}{\Delta R}\right)+\frac{\pi}{2}\right)=\cos \left(\alpha_{\max }+\varphi+\frac{\pi}{2}\right)
$$

and all discrete quadrature points with $\left|\mu_{i}\right| \leq\left|\mu_{\max }\right|$. For all of these quadrature points $\mu_{i}$, the distance $r_{i}$ between disk atmosphere surface and stellar surface and the projected stellar surface fraction $A_{i}^{\text {proj }}$ are calculated. The latter is determined taking into account the integration weight $a_{i}$ for each $\mu_{i}$. Each area $A_{i}^{\text {proj }}$ is subdivided and a mean intensity reaching the disk surface is evaluated using

- a blackbody spectrum to represent the stellar intensity

$$
I_{\nu}^{-}\left(\mu_{i}, \nu\right)=\frac{B_{\nu}}{2 \pi}\left(\sum_{j=1}^{\mathrm{NS}} A_{j}\left(\frac{R_{\star}}{r_{j}}\right)^{2} \frac{I_{\nu}\left(\mu_{j}, \nu\right)}{I_{\nu}(1, \nu)}\right)
$$

- a PHOENIX spectrum as input

$$
I_{\nu}^{-}\left(\mu_{i}, \lambda\right)=\frac{1}{2 \pi}\left(\sum_{j=1}^{\mathrm{NS}} A_{j}\left(\frac{R_{\star}}{r_{j}}\right)^{2} I_{\mathrm{Sp}}\left(\mu_{j}, \lambda\right)\right)
$$

where $A_{j}=A_{j}^{\text {proj }} / \pi R_{\star}^{2}$, NS is the number of surface segments for quadrature point $\mu_{i}$, and $\mu_{j}$ the angle under which the radiation leaves the stellar surface. The factor $1 / 2 \pi$ is needed since the irradiation onto the disk is unidirectional and not isotropic. In the case of a blackbody representation of the star, limb darkening is taken into account by the factor $I_{\nu}\left(\mu_{j}, \nu\right) / I_{\nu}(1, \nu)$ which is taken from Claret (2004).

\section{$2.9 \quad$ Energy conservation}

The standard accretion disk model demands that all the energy that is produced by viscous dissipation, i.e. mechanical energy, is released in form of radiative and convective energy, viz.

$$
E_{\mathrm{m}}(m)=E_{\mathrm{r}}(m)+E_{\mathrm{c}}(m)
$$

For a viscous disk, the left hand term can be written as

$$
E_{\mathrm{m}}(m)=\frac{9}{4} \frac{G M_{\star}}{R^{3}} \nu(m) \varrho(m)
$$



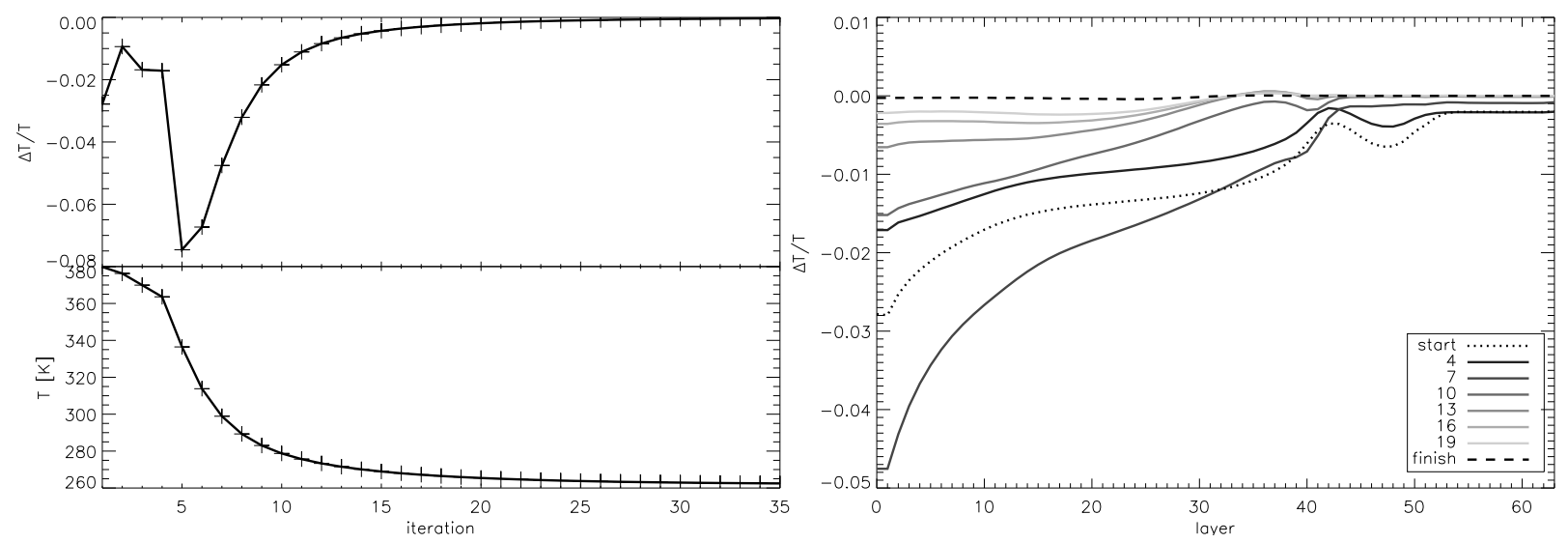

Figure 2.6: Temperature correction scheme for a not irradiated disk atmosphere with $R=0.069 \mathrm{AU}, \dot{M}=5 \times 10^{-9} M_{\odot} / \mathrm{yr}, R e=5 \times 10^{4}, R_{\star}=1.57 R_{\odot}$, and $M_{\star}=0.6 M_{\odot}$. The top plot of the left panel shows the relative temperature change for each iteration in the top most layer. The bottom plot is the temperature in that layer. The right plot shows relative temperature corrections as a function of layer number for a chosen set of iteration steps (see legend). The dotted line shows the correction in the first and the dashed line in the last iteration. The correction is limited to $\Delta T / 4$ in the first four iterations to avoid overcorrections. In this example convergence in the Unsöld-Lucy temperature correction is reached after $\sim 35$ iterations.

(Kriz \& Hubenv, 1986) and the radiative energy is expressed by

$$
E_{\mathrm{r}}(m)=4 \pi \int_{0}^{\infty}\left(\eta_{\nu}(m)-\chi_{\nu}(m) J_{\nu}(m)\right) d \nu
$$

The convective energy term $E_{\mathrm{c}}$ is neglected from here on because radiation is dominating the temperature structure and convection seems to have little effect (D'Alessio et al., 1998).

An Unsöld-Lucy class temperature correction scheme (Lucv, 1964; Dreizler, 2003) is set up by integrating the second frequency-integrated moment of the radiative transfer equation

$$
\frac{d K}{d m}=\kappa_{H} H
$$

where

$$
\kappa_{H}=\int_{0}^{\infty}\left(\chi_{\nu} / \varrho\right) H_{\nu} d \nu / H
$$

which yields for the $K$-correction $\Delta K=K_{i+1}-K_{i}$ between iteration $i$ and $i+1$

$$
\Delta K(m)=\Delta K(0)+\int_{0}^{m} \kappa_{H} \Delta H\left(m^{\prime}\right) d m^{\prime} .
$$

With the introduction of the Eddington factors

$$
f_{K}=\frac{K}{J} \quad \text { and } \quad f_{H}=\frac{H(0)}{J(0)}
$$


Eq. (2.54) can be rewritten as a correction in $\mathrm{J}$ :

$$
\Delta J(m)=\frac{f_{K}(0) \Delta H(0)}{f_{K} f_{H}}+\frac{1}{f_{K}} \int_{0}^{m} \kappa_{H} \Delta H\left(m^{\prime}\right) d m^{\prime}
$$

and insertion of the first frequency-integrated moment of the radiative transfer equation

$$
\frac{d H}{d m}=\kappa_{J} J-\kappa_{B} B
$$

and the Stefan-Boltzmann law

$$
B=\frac{4 \sigma}{\pi} T^{4}
$$

gives the temperature correction law

$$
\Delta T=\frac{\pi}{4 \sigma T^{3}}\left[\frac{\kappa_{J}}{\kappa_{B}}\left(\frac{\Delta H(0) f_{K}(0)}{f_{K} f_{H}}+\frac{1}{f_{K}} \int_{0}^{m} \kappa_{H} \Delta H\left(m^{\prime}\right) d m^{\prime}\right)-\frac{1}{\kappa_{B}} \frac{d \Delta H(m)}{d m}\right]
$$

with

$$
\begin{aligned}
& \kappa_{J}=\frac{1}{J} \int_{0}^{\infty}\left(\kappa_{\nu} / \varrho\right) J_{\nu} d \nu, \\
& \kappa_{B}=\frac{1}{B} \int_{0}^{\infty}\left(\kappa_{\nu} / \varrho\right) B_{\nu} d \nu,
\end{aligned}
$$

the absorption mean and Planck mean opacity. An example iteration history is shown in Fig. 2.6. In Eq. (2.59) $\Delta H=H_{\text {target }}-H_{\text {int }}$, i.e. the difference between the dissipated flux in the disk at a given layer plus the flux coming from the central star and the internal flux. If energy conservation is reached, all the flux that is generated by external and mechanical heating is balanced by the internal flux and the flux correction term $\Delta H$ vanishes. The derivative of the flux difference can be split according to

$$
\begin{aligned}
\frac{d \Delta H}{d m} & =\frac{d H_{\mathrm{m}}}{d m}-\frac{d H}{d m} \\
& =-\frac{9}{16} \frac{G M_{\star}}{R^{3}} \nu(m)-\left(\kappa_{J} J-\kappa_{B} B\right) .
\end{aligned}
$$

All together, the temperature correction equation reads

$$
\begin{aligned}
\Delta T & =\frac{\pi}{4 \sigma T^{3}}\left[\frac{1}{\kappa_{B}}\left(\frac{9}{16} \frac{G M_{\star}}{R^{3}} \nu(m)+d_{1}\left(\kappa_{J} J-\kappa_{B} B\right)\right)\right. \\
& \left.+d_{2} \frac{\kappa_{J}}{\kappa_{B}}\left(\frac{\Delta H(0) f_{K}(0)}{f_{K} f_{H}}+\frac{1}{f_{K}} \int_{0}^{m} \kappa_{H} \Delta H\left(m^{\prime}\right) d m^{\prime}\right)\right]
\end{aligned}
$$

where the damping factors

$$
d_{1}=e^{-\tau / \tau_{0}} \quad \text { and } \quad d_{2}=1-e^{-\tau / \tau_{0}}
$$

have been introduced in order to weight the different correction terms in cases where convergence problems occur. The parameter $\tau_{0}$ shifts the influence of the correction terms to a given optical depth. The factor $d_{1}$ controls the term which is responsible for local energy conservation. In dense 


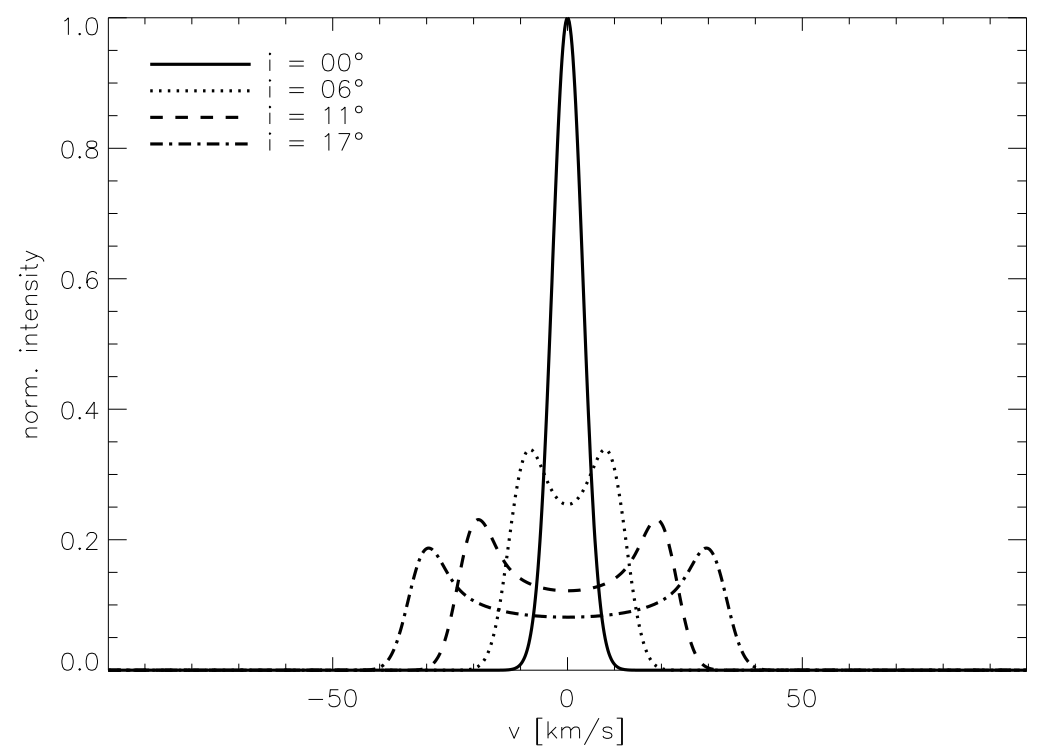

Figure 2.7: Line broadening due to rotation of disk matter for a model line of Gaussian shape. The unbroadened line (solid line) corresponds to a face-on $i=0^{\circ}$ view. The other lines are calculated for a view of the disk annulus with $R_{\text {in }}=0.07 \mathrm{AU}$ and $R_{\text {out }}=0.08 \mathrm{AU}$ under various angles $i$.

and optically thick regions of the atmosphere this term becomes small and does not contribute much to the temperature correction. The factor $d_{2}$ influences the sum of the terms that ensure the correct surface flux and global energy conservation.

One problem observed during the calculation of disk ring models was the oscillating behaviour of the temperature correction method. This behaviour could be overcome with the introduction of a global correction damping. A damping constant $i_{\text {damp }}$ supplied by the user on input limits the corrections in $\Delta B$ by $2^{-i_{\mathrm{damp}}}$. This limiting correction method had to be applied in most of the model calculations presented here.

\subsection{Line profiles}

After computing a set of self-consistent disk ring structures, a last radiative transfer iteration with a larger number of quadrature points (usually $N G=16$ ) and a fine wavelength spacing (up to $\Delta \lambda=0.2 \AA$ in the wavelength range of interest) is calculated to obtain a high-resolution model spectrum. Since the Keplerian rotation velocity and the inclination angle $i$ of the disk (only the projected surface fraction can be observed) are not considered in the single ring spectra yet, a combined spectrum is computed according to

$$
\begin{aligned}
\bar{I}_{\nu}(\nu, i) & =\cos (i) \int_{R^{\text {in }}}^{R^{\text {out }}} \int_{0}^{2 \pi} I(\nu, \phi, r, i) r d \phi d r \\
& =\pi \cos (i) \sum_{j=1}^{\mathrm{NR}}\left\{\left[\left(R_{j}^{\text {out }}\right)^{2}-\left(R_{j}^{\text {in }}\right)^{2}\right] \cdot \sum_{k=1}^{\mathrm{NA}} I\left(\nu^{\prime}\left(i, \phi_{k}\right), \phi_{k}, R_{j}, i\right)\right\} .
\end{aligned}
$$

In Eq. (2.66) it is assumed that the disk is axis-symmetric and that the intensity is constant 
for the whole annulus between inner and outer radii $R_{\text {in }}$ and $R_{\text {out }}$. In addition to the integration over all disk rings, the influence of the disk's rotation on the line profile is taken into account by applying the Doppler shift

$$
\nu^{\prime}(i, \phi)=\nu \sqrt{\frac{1-u(i, \phi) / c}{1+u(i, \phi) / c}}
$$

to the line. Here the velocity $u(i, \phi)=u_{\mathrm{Kep}} \sin (i) \cos (\phi)$ is determined for a given inclination $i$ and for a set of disk ring segments with azimuthal angle $\phi$. NA $=100$ disk ring segments are used, i.e. 100 steps in $\phi$, to determine the rotationally broadened line profile. This method is a simple way to determine line profiles for rotating accretion disks if the lines originate in the very upper layers of the disk. However, Horne \& Marsh (1986) noted that an anisotropy in the local emission pattern changes the global line shape in the case of unblended lines: line photons are trapped in optically thick emission layers and can more easily escape in directions where there are larger Doppler gradients. The true consequence of this on the line shape can only be analysed by means of full 3D radiative transfer calculations in rotating accretion disks.

\subsection{Parameter studies}

The complex system of structure equations is controlled by some input parameters which are listed in detail in the Appendix of this work. In order to better understand the influence of these parameters on the disk structure, a study for the most important parameters is presented in this section.

\subsubsection{Mass accretion rate}

One of the most important parameters controlling the standard accretion disk model is the mass accretion rate, i.e. the amount of material going through a disk annulus in a given time range. The total column mass density follows $\Sigma \propto \dot{M}$. Hence, for a constant viscosity, material is added to each part of the disk with increasing mass accretion rate. As shown in Eq. (2.8), the effective temperature of a disk annulus follows the relation $T_{\text {eff }} \propto \dot{M}^{1 / 4}$. The disk surface temperature is dominated by the stellar irradiation.

In order to illustrate the effect of the input parameter mass accretion rate $\dot{M}$ on the disk structure and line spectrum, disk ring structures for $R=0.075 \mathrm{AU}, R e=10^{6}, M_{\star}=1 M_{\odot}$, and $R_{\star}=2 R_{\odot}$ have been calculated. The outer minimal column mass is set to $m_{1}=10^{-4} \mathrm{~g} \mathrm{~cm}^{-2}$. In Fig. 2.8 temperature profiles for disks with different mass accretion rates are shown. The temperature close to the surface is mainly set by stellar irradiation. Only a small offset with $\dot{M}$ can be seen which is a result of the non-vanishing viscosity $\nu(m)$ (see Eq. (2.16) ) and the change in disk height which in turn influences the amount of stellar irradiation absorbed by the annulus. At $m \gtrsim 10^{-1} \mathrm{~g} \mathrm{~cm}^{-2}$ the influence of mechanical heating takes over the heating due to irradiation. In this case, the higher the accretion rate the hotter the material. This can be easily derived in optically thick regions $(\tau \gg 1)$ where Eq. (2.18) simplifies to

$$
T^{4}=\frac{3}{4} T_{\text {eff }}^{4}\left(\tau+\frac{1}{\sqrt{3}}\right)
$$

(Hubenv, 1990). With increasing $T_{\text {eff }}$ and optical depth $\tau$ (see Fig. 2.9) the local temperature also increases. A comparison to the temperature run for the optical thick case calculated from 


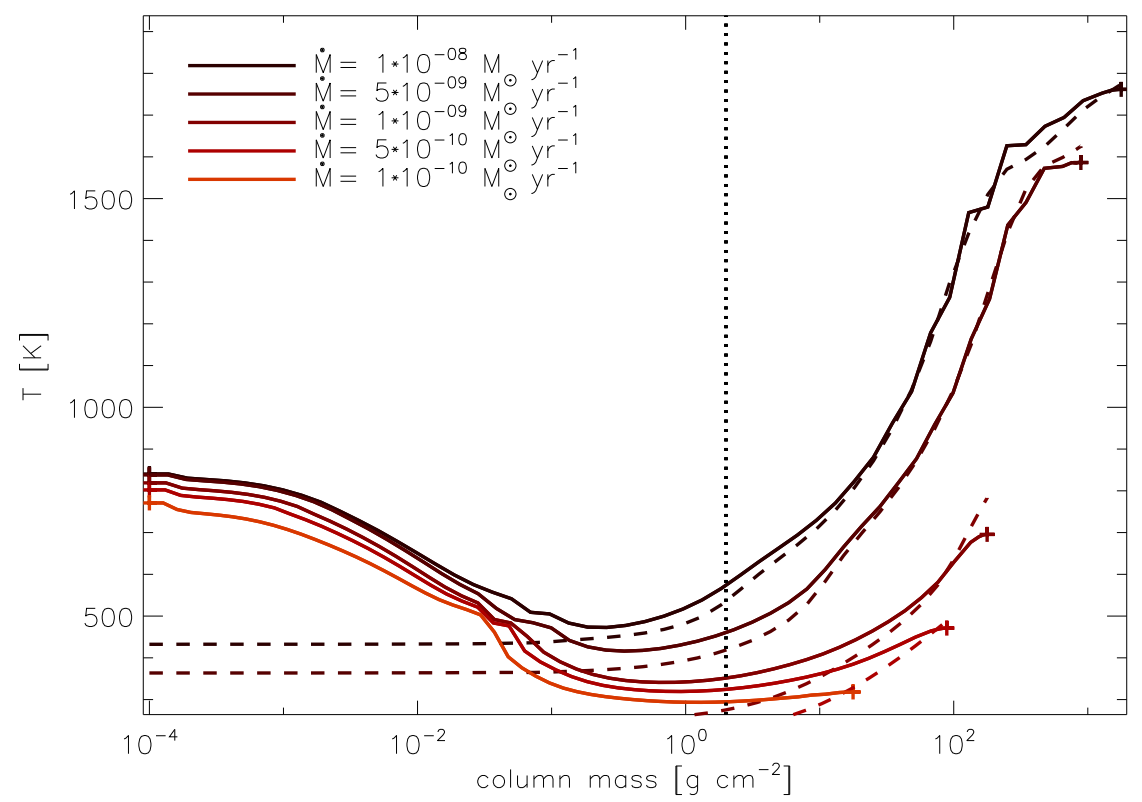

Figure 2.8: Temperature profiles for disk rings with $R=0.075 \mathrm{AU}, R e=5 \times 10^{4}$, $M_{\star}=1 M_{\odot}, R_{\star}=2 R_{\odot}$, and varying mass accretion rate (solid lines). The dashed line show simple temperature profiles calculated from Eq. (2.68) with $\tau=\tau_{\text {ross }}$. The dashed line marks the region with $\tau_{\text {ross }} \approx 1$, i.e. where the continuum becomes optically thick.

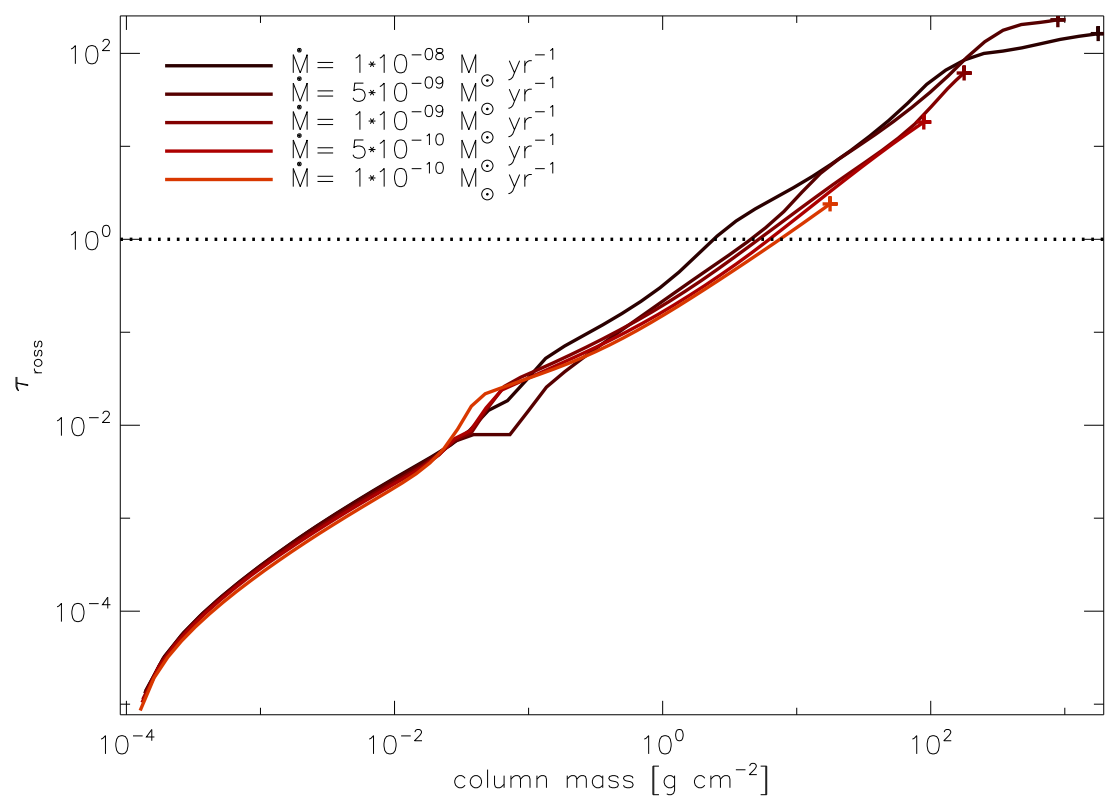

Figure 2.9: Rosseland optical depth profiles for models with different mass accretion rates. The dotted line marks $\tau_{\text {ross }}=1$. The higher the mass accretion rate the larger the optical depth at the midplane because the column mass density at the midplane scales linearly with $\dot{M}$. 


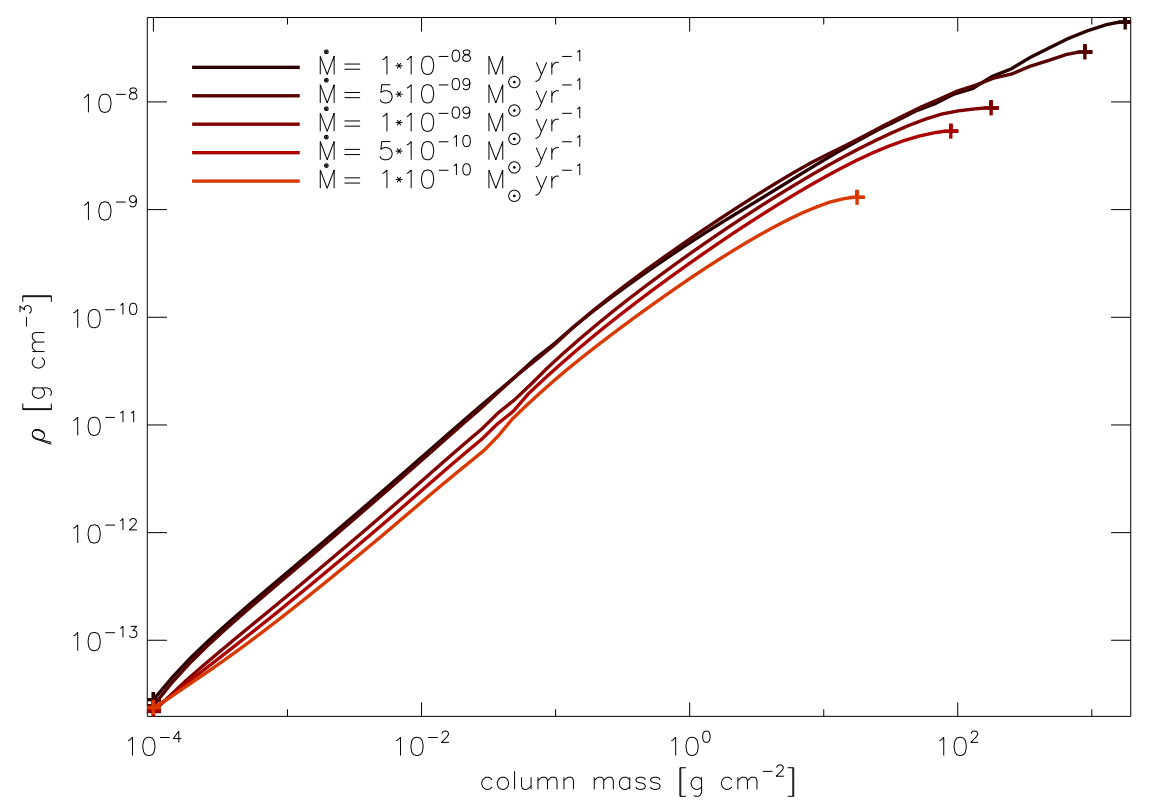

Figure 2.10: Density profiles for models with different mass accretion rates. The higher the mass accretion rate the larger the density at the midplane because the column mass density at the midplane scales linearly with $\dot{M}$.

Eq. (2.68) shows that the final irradiated model temperature does not deviate significantly for $\tau_{\text {ross }}>1$. Stratifications of optical depth for models with different mass accretion rate are plotted in Fig. 2.9] It shows that the higher $\dot{M}$, i.e. the higher $M_{0}$, the larger $\tau_{\text {ross }}$ at the midplane because there simply is more matter for higher $\dot{M}$ which can absorb radiation. The density profiles are plotted in Fig. 2.10, For a given column mass density $m$ the density increases slightly with increasing mass accretion rate.

Figure 2.11 shows the fundamental CO $v=1-0$ (R7) line calculated from the models with different mass accretion rates. From the non-normalised spectrum one sees that the continuum flux level increases strongly with $\dot{M}$. This can be explained by the relation $T_{\text {eff }} \propto \dot{M}^{1 / 4}$ and $I_{\text {cont }} \approx B\left[T_{\text {eff }}\right]$. The absolute line flux above the continuum is comparable for all five models because it is set by the temperature where the line becomes optically thick, which is in the irradiation dominated outer part of the atmosphere. Here the temperature is similar for all models. The relative strength of the line profiles becomes very different when the spectra are normalised. Now the line from the model with the smallest mass accretion rate is the strongest. This can be interpreted as a behaviour of contrast between continuum flux and line flux.

\subsubsection{Reynolds number}

The Reynolds number is the ratio of inertial forces to viscous forces, hence, $R e \propto \bar{\nu}^{-1}$. Furthermore, $M_{0} \propto R e$ (see Eq. (2.15)) which leads to a temperature increase near the midplane for models with increasing Reynolds number. However, since the effective temperature is independent of the Reynolds number, the local temperature does not increase for models with different $R e$ values at constant $m$ (except for the boundaries). Plots of the optical depth $\tau_{\text {ross }}$ and the density $\rho$ are shown in Figs. 2.13 and 2.14. The behaviour of the models with increasing Reynolds 

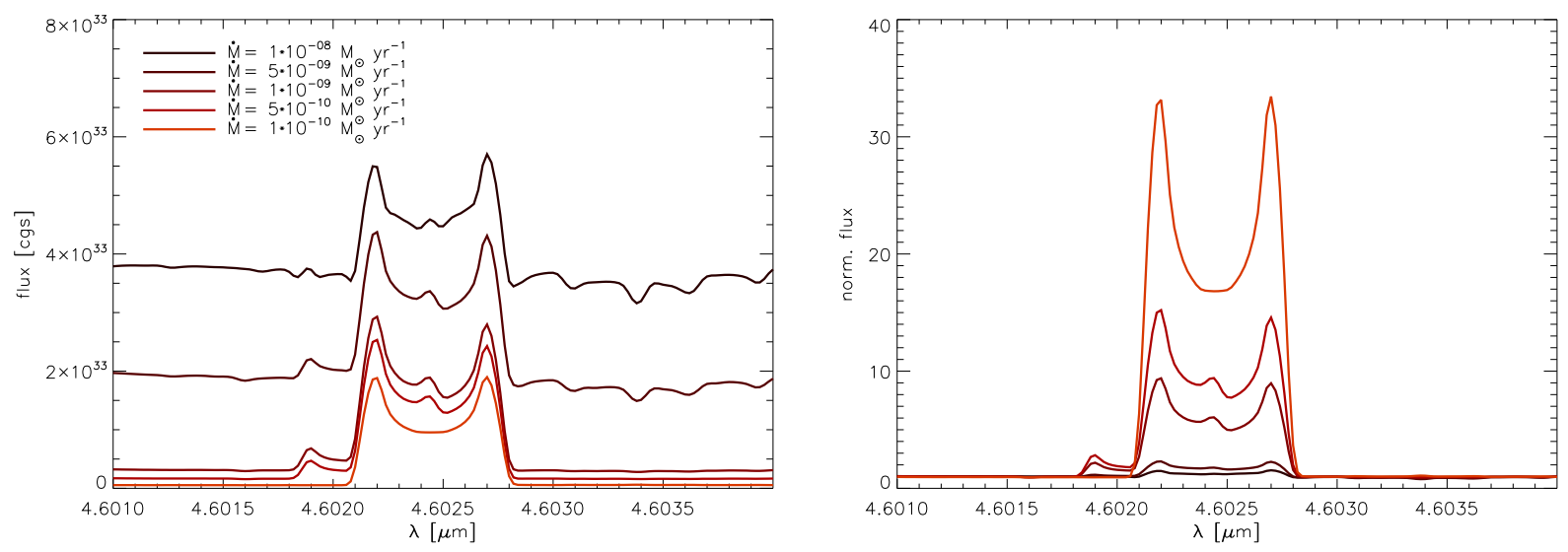

Figure 2.11: Line profiles for the fundamental CO $v=1-0(\mathrm{R} 7)$ line calculated from the models with different mass accretion rates. The left plot shows the total flux of the disk ring, the right plot the normalised flux. The assumed inclination is $i=10^{\circ}$ and the $v=1-0$ (R7) line is blended by a weaker CO $v=2-1$ (R15) line.

number is quite similar to that of the models in the previous Section with increasing $\dot{M}$ because in both cases the plotted values are influenced by the total column mass density $M_{0} \propto \dot{M} R e$.

Figure 2.15 shows the fundamental CO $v=1-0$ (R7) line calculated from the models with different Reynolds numbers. In the plot with the non-normalised spectrum section, the continuum flux level is different and only seems to converge towards a constant value for $R e>10^{6}$. For values $R e<10^{6}$ the continuum is not optically thick yet. Once the models becomes optically thick, a further increase of the Reynolds number will not be measurable in the spectrum (the models for $R e=10^{6}$ and $R e=10^{7}$ are actually overlapping). Therefore, the mass of a disk section cannot be determined once the disk is optically thick.

In the models presented Figs. 2.12 2.15] a viscosity that decreases with height $\nu(m) \propto$ $\bar{\nu}(m / M)$ has been assumed. This parametrisation may not be correct if for instance the dissipation is driven by turbulence caused by interaction of a magnetic field with ionised matter which is likely stronger ionised in the outer than in the inner disk layers. Therefore, test calculations for viscosities with different parameters $\zeta$ (see Eq. (2.16) ) were carried out. The differences in temperature and flux for a model with $R=0.075 \mathrm{AU}, \dot{M}=10^{-9} M_{\odot} \mathrm{yr}^{-1}, R e=10^{6}$, $M_{\star}=1 M_{\odot}$, and $R_{\star}=2 R_{\odot}$ are marginal when changing $\zeta$ from 1 (viscosity drops linearly with column mass) to $10^{-3}$ ( constant viscosity). This is also true for more optically thin models, i.e. when setting $R e=5 \times 10^{4}$. The independence of the temperature structure on $\zeta$ becomes evident when looking at Eqs. (2.16) and (2.50) because at the midplane the viscosity is not altered significantly for $\zeta<1$ and at the top of the atmosphere the liberated energy due to viscous dissipation is proportional to the density which is small.

\subsubsection{Stellar radius}

As described in this Chapter, models of circumstellar disks depend on a number of input parameters. In the case of the classical stellar atmosphere problem, the models are sufficiently described by the effective temperature, the surface gravity, and the metallicity. For circumstellar disks, one needs to set the disk parameters (Reynolds number, mass accretion rate, inner and outer radius for the simulated region, inclination of the disk, etc.) as well as the stellar parameters (mass, radius, and effective temperature) which control the irradiation field. Therefore, in order to reduce 


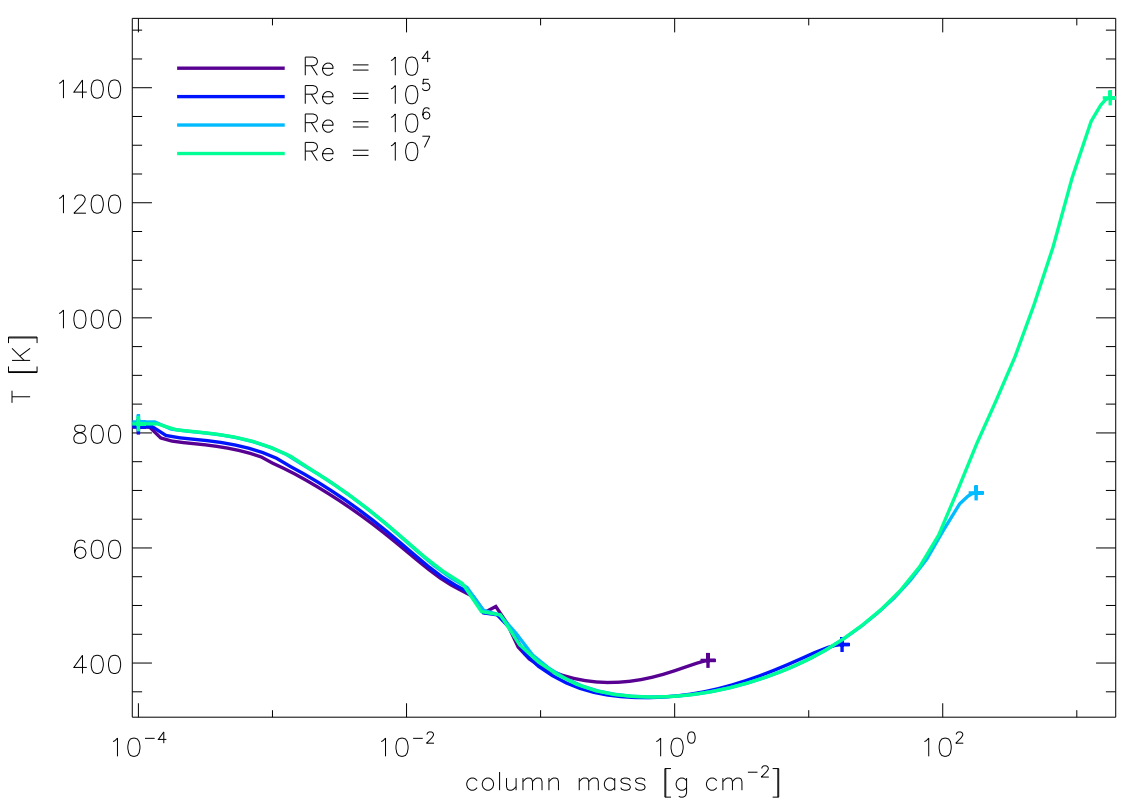

Figure 2.12: Temperature profile for disk rings with $R=0.075 \mathrm{AU}, \dot{M}=10^{-9} M_{\odot} \mathrm{yr}^{-1}$, $M_{\star}=1 M_{\odot}, R_{\star}=2 R_{\odot}$, and varying Reynolds number. The higher the Reynolds number, the more mass is present in the region and heated due to dissipation close to the midplane. The outer temperature is set by the stellar irradiation and only varies very little for different Reynolds numbers.

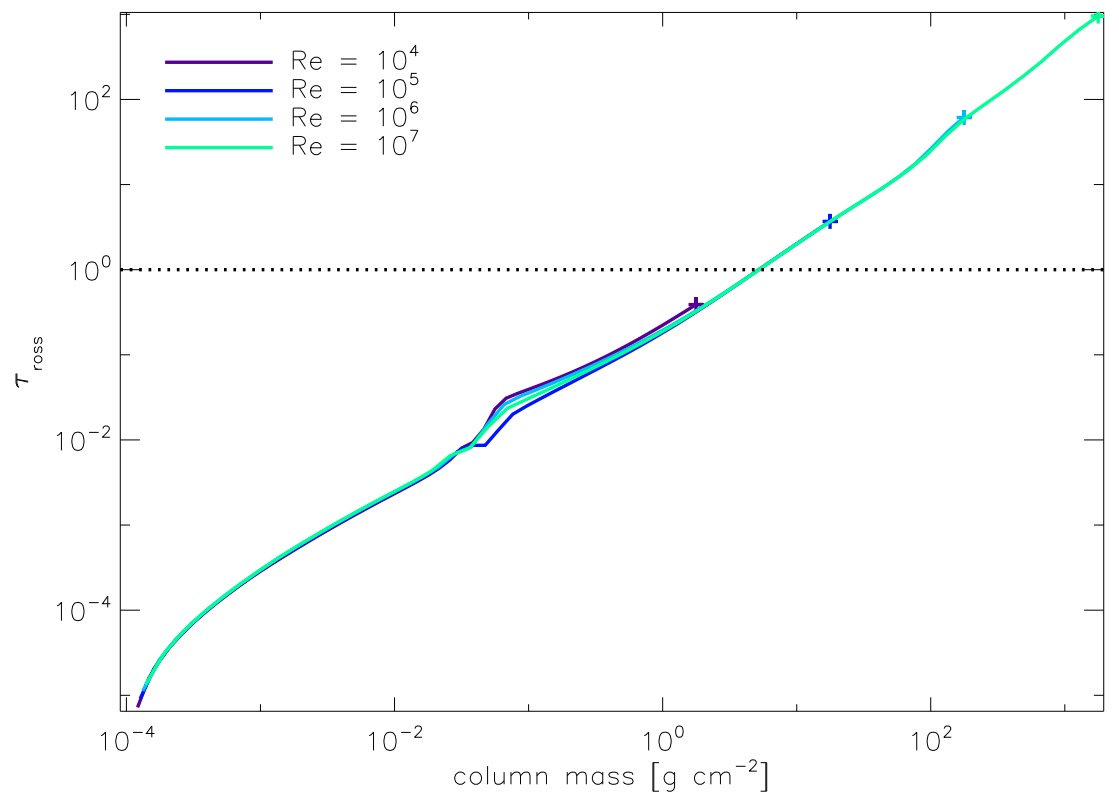

Figure 2.13: Rosseland optical depth profiles for models with different Reynold numbers. The dashed line marks $\tau_{\text {ross }}=1$. The model with $R e=10^{4}$ does not become optically thick. 


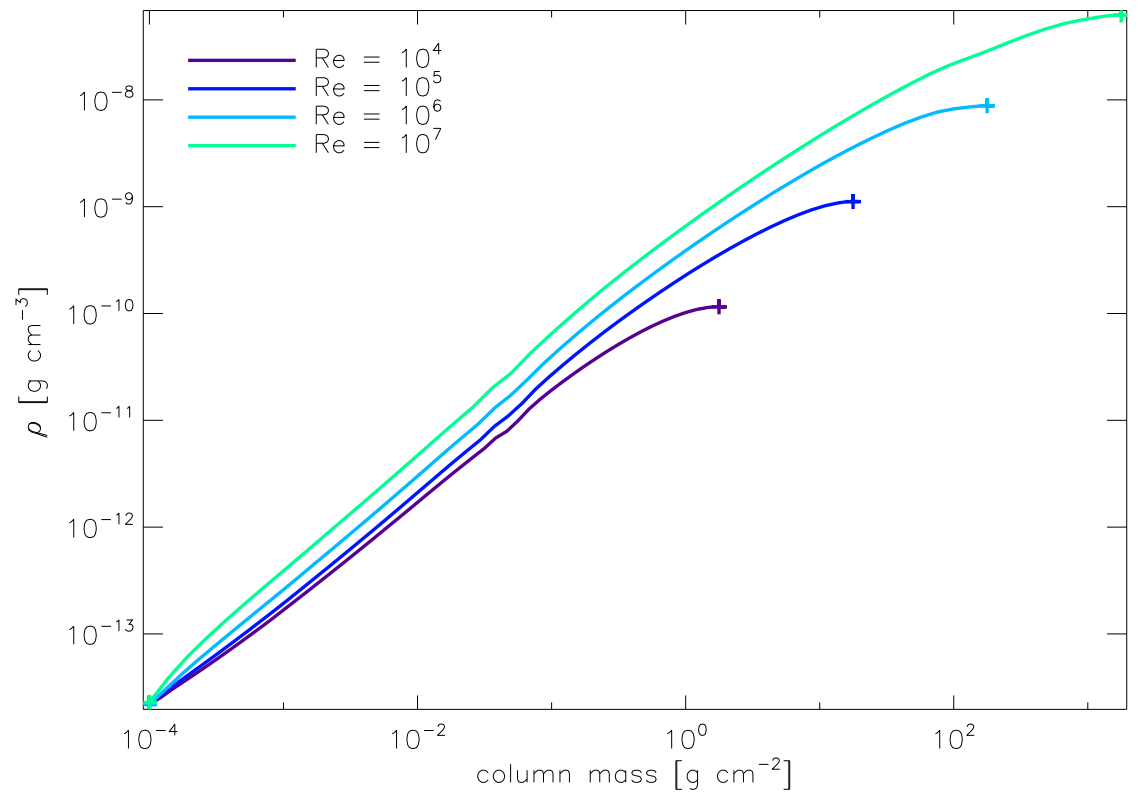

Figure 2.14: Density profiles for models with different Reynold numbers. The gradient in $\rho$ becomes zero close to the midplane because of symmetry.
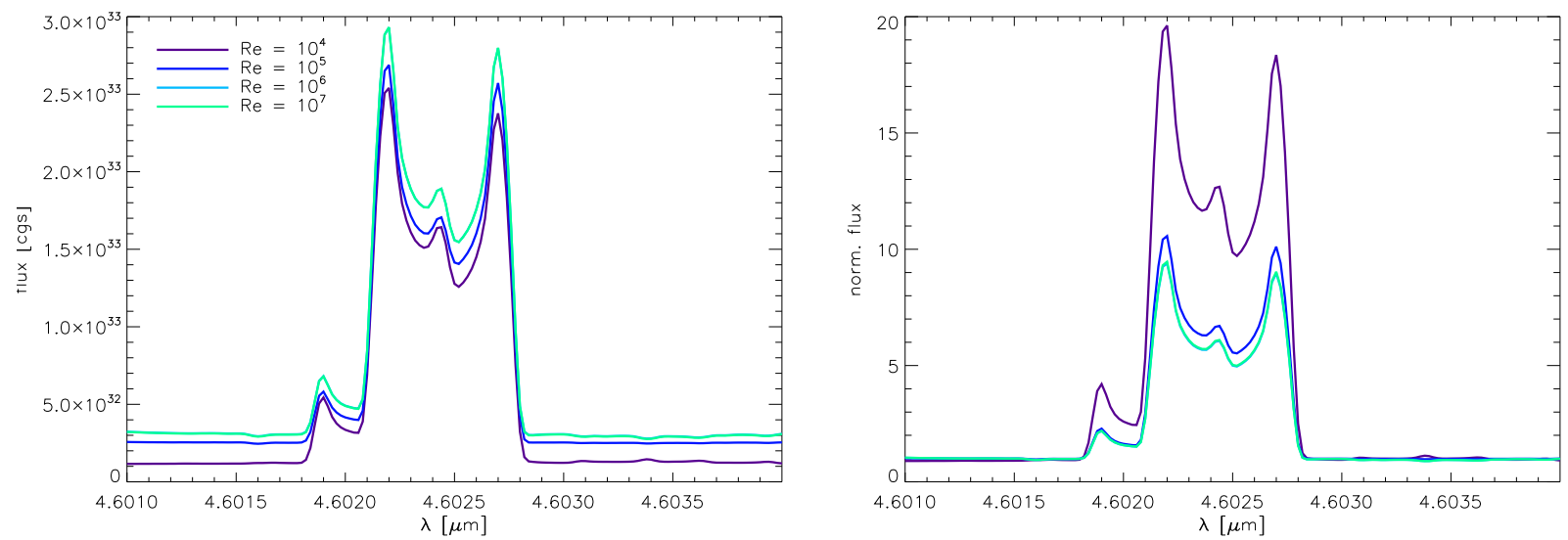

Figure 2.15: Line profiles for the fundamental CO $v=1-0$ (R7) line calculated from the models with different Reynolds numbers. The continuum flux level converges towards a constant value for models with $R e>10^{6}$. The assumed inclination is $i=10^{\circ}$ and the $v=1-0$ (R7) line is blended by a weaker CO $v=2-1$ (R15) line.

the number of free parameters for model calculations for an observation of a certain object, it is very helpful if the stellar parameters are well constrained. The influence of the stellar radius on the temperature structure and the line flux is shown in Figs. 2.16 and 2.17 for models with $R=0.075 \mathrm{AU}$ (and $R=0.150 \mathrm{AU}$ for the $T$-structures), $\dot{M}=10^{-9} M_{\odot} \mathrm{yr}^{-1}, M_{\star}=1 M_{\odot}$, and $R e=10^{6}$. The stellar radius was set to $R_{\star}=1 R_{\odot}, 2 R_{\odot}$, and $3 R_{\odot}$ while the stellar effective temperature is kept constant at $T_{\text {eff }}=4000 \mathrm{~K}$. An increase in radius at constant $T_{\text {eff }}$ implicates a rise in luminosity and influences the irradiation geometry of the star-disk system.

Figure 2.16] shows that the influence of the stellar radius on the vertical temperature structure 

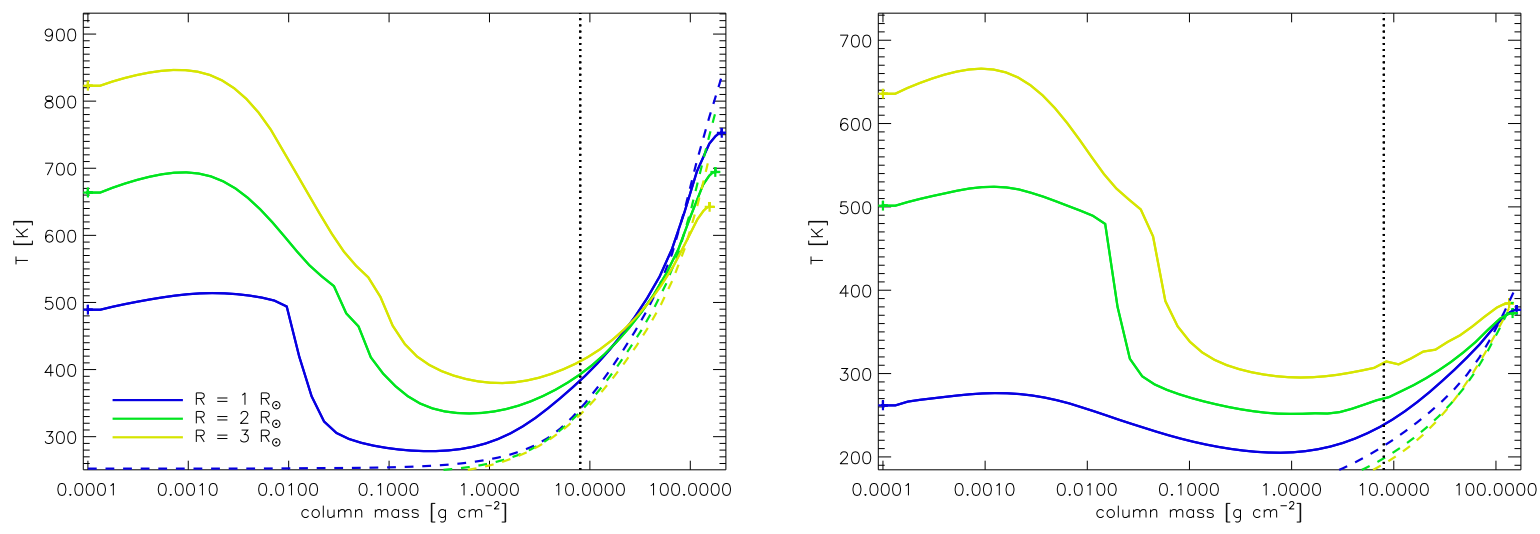

Figure 2.16: Temperature profile for disk rings with $R=0.075 \mathrm{AU}$ (left) and $R=$ $0.150 \mathrm{AU}$ (right), $\dot{M}=10^{-9} M_{\odot} \mathrm{yr}^{-1}, M_{\star}=1 M_{\odot}, R e=10^{6}$, and varying stellar radius. The dotted line marks the height where $\tau_{\text {cont }} \approx 1$. The dashed coloured lines give the run of the temperature according to Eq. (2.68).

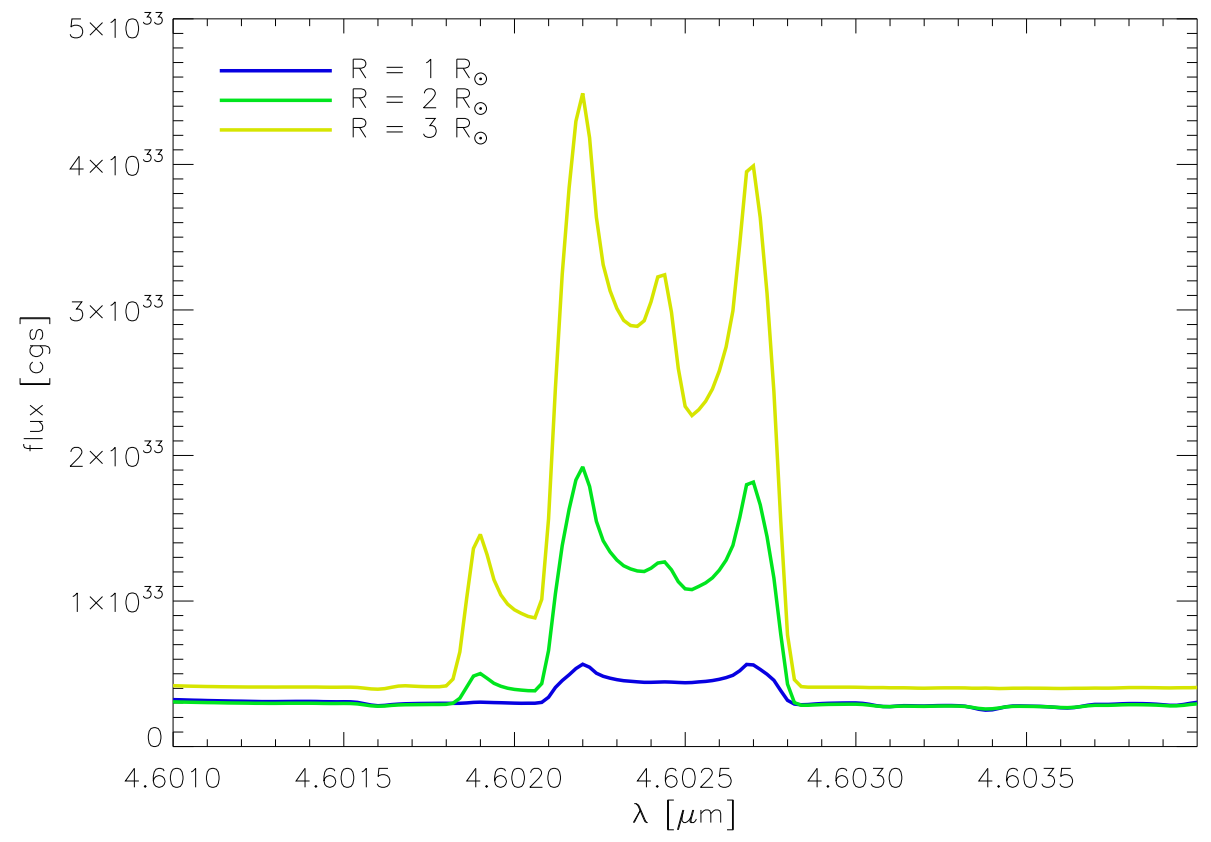

Figure 2.17: Line profiles for the fundamental CO $v=1-0$ (R7) line calculated from the models with different stellar radii at a distance of $R=0.075$ AU from the central star. The assumed inclination is $i=10^{\circ}$ and the $v=1-0(\mathrm{R} 7)$ line is blended by a weaker CO $v=2-1$ (R15) line.

of the disk is significant. In the outermost strongly irradiated layers, the temperature raises from $\sim 500 \mathrm{~K}$ to $\sim 700 \mathrm{~K}$ when the radius is increased from $1 R_{\odot}$ to $2 R_{\odot}$ and even up to $\sim 850 \mathrm{~K}$ for $3 R_{\odot}$ in the $R=0.075 \mathrm{AU}$ model. For the $R=0.150 \mathrm{AU}$ model the relative jump in $T$ in the outer layers is even stronger. This temperature increase occurs in a region where the spectral lines, e.g. CO fundamental transitions, become optically thick. Hence, the lines become stronger. The region around $\tau_{\text {ross }}=1$ (see the dotted line in Fig. 2.16) is shielded by the outer layers and 
only mildly heated. As a result, the flux level of the continuum is very similar for all three models with different stellar radii (see Fig. 2.17). The line strength, i.e. the ratio of line to continuum flux, can thus be described as a result of the contrast between temperatures in the irradiation dominated outer layers of the disk and the accretion dominated inner regions of the disk. This again shows how important the treatment of the irradiation geometry is for the calculation and determination of a best fit model. 


\subsection{Contribution function}

The contribution function describes how much each atmospheric layer contributes to the total flux. Therefore, it is a convenient tool to analyse the depth of line and continuum formation. It also allows to determine if the cut-off of the atmosphere at a certain minimum column mass $m_{1}$ is well chosen.

The frequency-dependent contribution function $C_{\nu}$ is defined by:

$$
C_{\nu}:=\frac{\Delta\left(I_{\nu} e^{-\tau_{\nu}}\right)}{\Delta z},
$$

where $I_{\nu} e^{-\tau_{\nu}}$ is the the intensity at frequency $\nu$ that can escape from a layer with an optical depth $\tau_{\nu}$. The difference between the escaping intensity of two neighbouring layers divided by the distance between those layers $\Delta z$ gives the contribution function from that region of the atmosphere. This model contribution can be compared with the theoretically expected one from the temperature structure and absorption coefficient

$$
C_{\nu}^{\exp }=S_{\nu} e^{-\tau_{\nu}} \alpha_{\nu},
$$

where $S_{\nu}\left(=B_{\nu}\right.$ in LTE) is the source function and $\alpha_{\nu}$ the absorption coefficient.

Figure 2.19 shows the line and the continuum contribution function for two models with $R=0.031 \mathrm{AU}$ and $R=0.094 \mathrm{AU}, 3 \times 10^{-9} M_{\odot} \mathrm{yr}^{-1}, R e=5 \times 10^{6}$, and $m_{1}=10^{-5} \mathrm{~g} \mathrm{~cm}^{-2}$ as well as their temperature structures. The line contribution functions agree very well with the theoretically expected one. The continuum contribution function for the model with $R=0.031 \mathrm{AU}$ shows somewhat smaller values at $\tau_{\text {cont }}<1$ which are compensated at $\tau_{\text {cont }}>1$. For the model with $R=0.094 \mathrm{AU}$, the line contribution function again agrees well with the expected one, the continuum contribution function, however, becomes negative at $\tau_{\text {cont }} \approx 0.1$. This behaviour cannot be explained by Eq. (2.70) which is always $\geq 0$. This seems to be an artifact of the poorly converged inner temperature structure in that particular model.

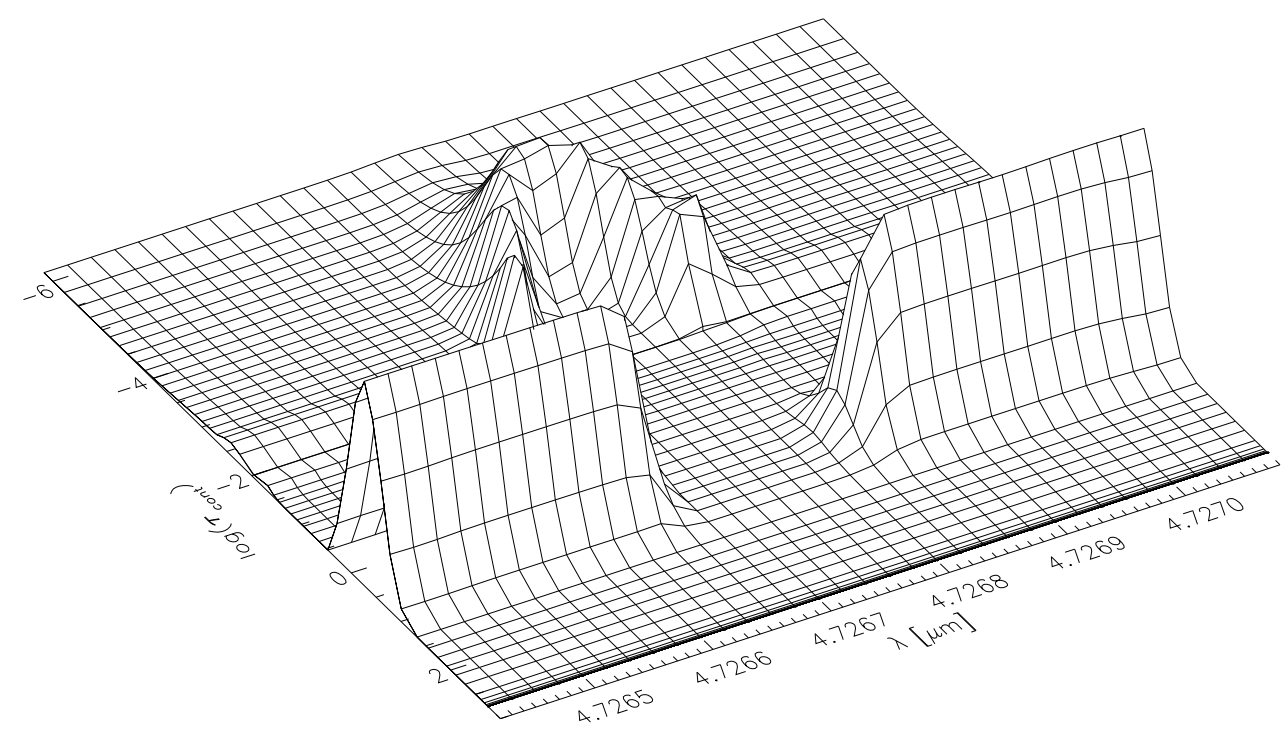

Figure 2.18: $3 \mathrm{D}$ wavelength-dependent contribution function of the continuum and the line around the fundamental CO $v=1-0(\mathrm{P} 7)$ line at $\lambda=4.72674 \mu \mathrm{m}$ from a model with $R=0.09 \mathrm{AU}$. It becomes nicely visible that the line wings originate significantly deeper in the atmosphere than the line core. 

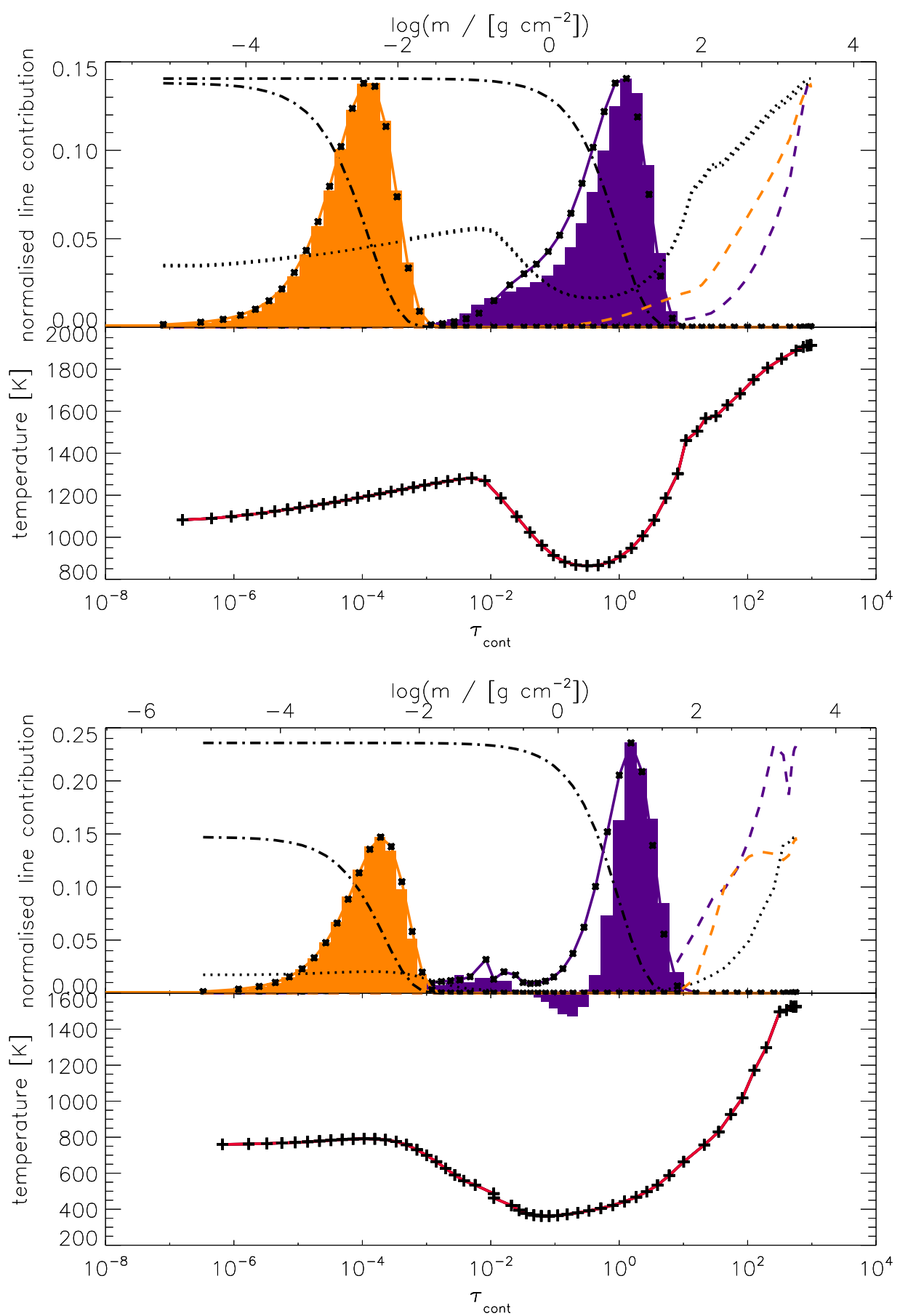

Figure 2.19: Top: The upper panel shows the line (orange bars) and continuum (purple bars) contribution functions derived from a model with $R=0.031 \mathrm{AU}, 3 \times 10^{-9} M_{\odot} \mathrm{yr}^{-1}$, $R e=5 \times 10^{6}$, and $m_{1}=10^{-5} \mathrm{~g} \mathrm{~cm}^{-2}$. The enveloping asterisks are the expected values from Eq. (2.70), the dotted line is the Planck function $B_{\nu}$, the dashed dotted line is the dilution factor $e^{-\tau_{\text {cont }}}$, and the coloured dashed lines the absorption coefficient $\alpha_{\nu}$ (all normalised to the plot range). The lower panel shows the run of the temperature. Bottom: The same as above for a model with $R=0.094$ AU. 


\section{Chapter 3}

\section{Spectral analyses}

The 1+1D accretion disk models that have been developed as part of this work are described in detail in Chapter 2. The dependence of the structure and the emergent spectrum on the various input parameters is shown in Sect. 2.11] In order to characterise the properties of protoplanetary disks, the models have to be compared with observations. For the analysis of the warm and hence molecule rich part of accretion disks, high-resolution IR spectra are very well suited because i) the disk matter with temperatures of $100-1500 \mathrm{~K}$ radiates mostly in the near- to mid-IR wavelength range and ii) spectroscopically resolved line profiles of inclined disks can be used to separate disk radii in velocity (see Sect. 2.10) due to differentially rotating matter.

The ESO VLT instrument CRIRES (CRyogenic high-resolution InfraRed Echelle Spectrograph; Kaeufl et al., 2004) provides high-resolution $(R \sim 100000)$ spectroscopic observations in the wavelength range between $0.95-5.40 \mu \mathrm{m}$. The large programme "The planet-forming zones of disks around solar-mass stars: a CRIRES evolutionary survey" (P.I. van Dishoeck) proposes the observation of 75 Spitzer-selected YSOs. The object selection ranges from deeply embedded stars $(\sim 0.1 \mathrm{Myr}$ in age $)$ to WTTS with disks that start to dissipate $(\sim 10 \mathrm{Myr})$. Three potentially interesting candidates have been selected for an analysis with the PHOENIX DISK model code.

\subsection{GQ Lup}

\subsubsection{System parameters}

GQ Lup is a classical T Tauri star (CTTS) which is mostly known for its recently discovered substellar companion GQ Lup B (Neuhäuser et al., 2005). The activity due to ongoing accretion of the CTTS makes a well constrained determination of its physical parameters difficult. This becomes obvious regarding the differences in visual brightness of more than 2 mag $\left(V_{\max }=11.33 \mathrm{mag}\right.$ and $V_{\min }=13.36 \mathrm{mag}$; Janson et al., 2006). Broeg et al. (2007) and Seperuelo Duarte et al. (2008) (BR07 and SD08 from here on) both obtained photometric and spectroscopic data to determine rotational periods and $v \sin i$ for GQ Lup A. From these parameters they derived stellar parameters which are needed as input for the DISK model calculations in order to keep the number of free fit parameters small. Both authors assume an effective temperature for a K7 V star of $T_{\text {eff }}=4060 \mathrm{~K}$ (Kenvon \& Hartmann, 1995).

SD08 obtained a radius of $R_{\star}=1.8 \pm 0.3 R_{\odot}$ for the $\mathrm{K} 7 \mathrm{~V}$ star assuming a mean distance of $150 \pm 20 \mathrm{pc}$ by comparing the observed optical flux of GQ Lup with that of the K7V standard HD157881. From evolutionary tracks they derive a mass of $M_{\star}=0.8 \pm 0.2 M_{\odot}$. Together with their rotational period of $10.7 \pm 1.6 \mathrm{~d}$ and a spectroscopically determined $v \sin i=6.5 \pm 2.0 \mathrm{~km} \mathrm{~s}^{-1}$ 
Table 3.1: Properties of GQ Lup A

\begin{tabular}{lccccc}
\hline \hline author & $d[\mathrm{pc}]$ & $P[\mathrm{~d}]$ & $v \sin i\left[\mathrm{~km} \mathrm{~s}^{-1}\right]$ & $R_{\star}\left[R_{\odot}\right]$ & incl. $\left[{ }^{\circ}\right]$ \\
\hline Broeg et al. (2007) & 140 & $8.45 \pm 0.2$ & $6.8 \pm 0.4$ & $2.55 \pm 0.41$ & $27 \pm 5$ \\
Seperuelo Duarte et al. (2008) & 150 & $10.7 \pm 1.6$ & $6.5 \pm 2.0$ & $1.80 \pm 0.30$ & $51 \pm 13$ \\
\hline \hline
\end{tabular}

they find an inclination angle of $i=51^{\circ} \pm 13^{\circ}$.

BR07 measure a shorter photometric period of $8.45 \pm 0.2 \mathrm{~d}$ and a larger radius of $R_{\star}=$ $2.55 \pm 0.41 R_{\odot}$ from $T_{\text {eff }}$ and a luminosity $L$ from evolutionary tracks. With these values and $v \sin i=6.8 \pm 0.4 \mathrm{~km} \mathrm{~s}^{-1}$ they predict an inclination angle of $i=27^{\circ} \pm 5^{\circ}$. The different measured and derived values are listed in Table 3.1

\subsubsection{Model grid}

For the results of BR07 and SD08 (essentially for the different stellar radii $R_{\star}$ derived by the two groups) small model grids were calculated with the following parameters:

- $\dot{M}=2 \times 10^{-8}-7 \times 10^{-10} M_{\odot} \mathrm{yr}^{-1}$

- $R e=5 \times 10^{4} / 1 \times 10^{5}$

- $M_{\star}=0.8 M_{\odot}$

- $T_{\text {eff }}=4060 \mathrm{~K}$

- $R_{\star}=1.80 R_{\odot} / 2.55 R_{\odot}$

- $R=0.031,0.045,0.065,0.094,0.137,0.200,0.290,0.422 \mathrm{AU}$

Free parameters in this analysis are therefore the mass accretion rate $\dot{M}$, the Reynolds number $R e$, the stellar radius $R_{\star}$, as well as the inner and outer radius of the disk $R_{\text {in }}$ and $R_{\text {out }}$. Furthermore, the slope of the disk height $\Delta z_{\max } / \Delta R$, which is needed to determine the irradiation angle, is not calculated self-consistently (see Sect. 2.8). Therefore, the slope needs to be adjusted by an additional input parameter to guarantee that the slope defined at the beginning of the model calculations is equal to the one determined hydrostatically taking irradiation into account. The dust grains are assumed to have an ISM type power law grain size distribution with $a_{0}=6.25 \times 10^{-3} \mu \mathrm{m}$ and an exponent of -3.5 . The abundances follow Grevesse \& Noels (1993).

The number of radial grid points has been chosen to be small in order to keep computational time low. The points are spaced equidistantly on a logarithmic $R$ grid in order to avoid large steps in $F^{-}$and $T_{\text {eff }}$. The simplification of a discrete number of radial grid points can only be justified if an increase in grid points does not change the emergent spectrum. Therefore, the effect of doubling the number of grid points from 8 to 16 on the total spectrum has been investigated for one input parameter set exemplarily and no significant changes greater than the systematical error made during normalisation has been measured.

Because the number of free parameters in the models and the CPU time for the computation of a disk model is large, a statistical evaluation of the models and a determination of the best-fit model by $\chi^{2}$-fitting techniques is difficult. Therefore, the analysis presented here is restricted to an interpretation by eye and a comparison between models with different parameters. Furthermore, the properties of disk rings with different radii $R$ are presented. 


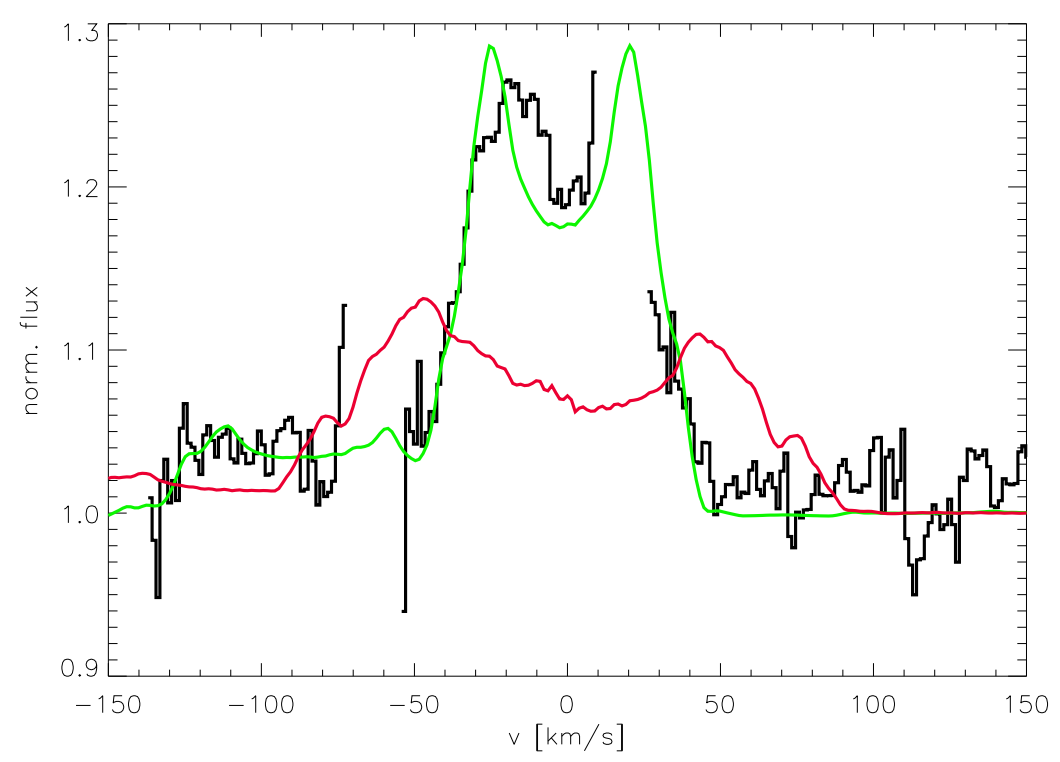

Figure 3.1: Comparison between observed CO line profile (black line) and models with $i=22^{\circ}$ (green line) and $i=51^{\circ}$ (red line). The two models are equivalent except for the different inclination angle. The line profile of the model with $i=51^{\circ}$ is too broad while the model with $i=22^{\circ}$ fits the line wings well.

\subsubsection{Inclination}

In order to rule out one of the proposed inclinations $i$, models with the different proposed parameters have been calculated (see Tab. 3.1). The smaller stellar radius derived by SD08 leads to a luminosity which is half as large as the one given by BR07. Therefore, the temperature set due to the stellar irradiation is smaller for the SD08 parameters and the lines are weaker (see Sect. 2.11.3). Since the total observed spectrum is a composition of the disk and the stellar flux, the emission lines are further diluted. In Fig. 3.1 the observed CO $v=1-0 \mathrm{P}(5)$ line is compared to models with $\dot{M}=3 \times 10^{-9} M_{\odot} \mathrm{yr}^{-1}, R e=5 \times 10^{4}, R_{\text {in }}=0.052 \mathrm{AU}, R_{\text {out }}=0.500 \mathrm{AU}$, and the stellar parameters of BR07. The green line shows the spectrum as it can be observed for a disk with an inclination of $i=22^{\circ}$. This is the lower limit of the value given by BR07 and it fits the line wings well. The line wings could be similarly well fit if the inclination is increased, which makes the line broader, and the inner radius is increased, which results in smaller rotational velocities and hence less strong broadening. However, the line would appear less strong in this case because the emerging intensity $I$ is smaller for large inclinations and an increase in $R_{\text {in }}$ yields a smaller radiating disk surface. Therefore, other parameters like mass accretion rate or Reynolds number would need to be adjusted in order to reproduce a good quality fit with $i>22^{\circ}$. The red line shows how the spectrum of the same model looks like if the inclination is changed from $i=22^{\circ}$ to $i=51^{\circ}$, i.e. the value proposed by SD08. The change in appearance of the CO line is quite dramatic. The line wings are too broad and the intensity is too weak. Even though this comparison is done for a model with the BR07 parameters that fits the observation well for $i=22^{\circ}$, the basic problem with a larger inclination as proposed by SD08 becomes clear. For a model disk with $i=51^{\circ}$, the inner radius would have to be at $R_{\text {in }}=0.200 \mathrm{AU}$ and the outer radius for the line (not the total disk) at $R_{\text {out }}=0.650 \mathrm{AU}$ to fit the line wings and the inner part 


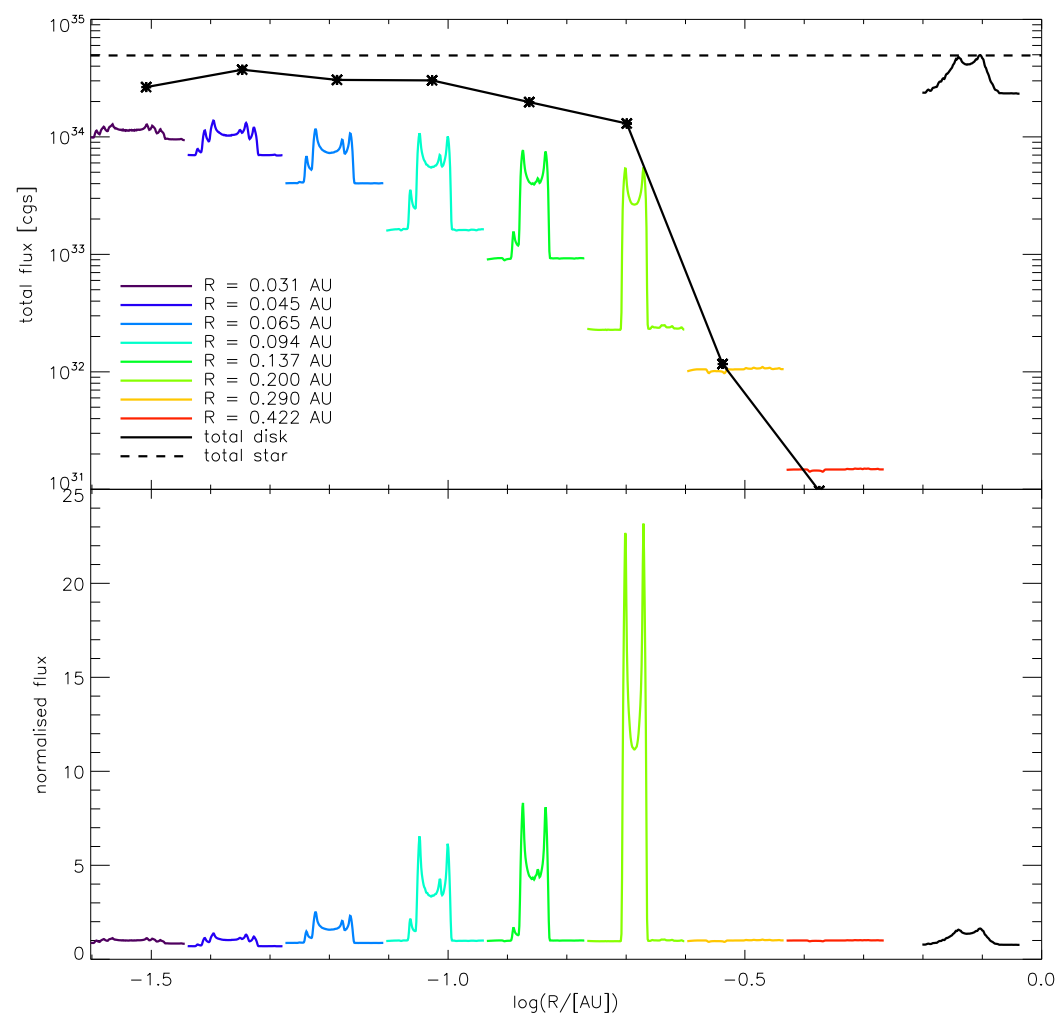

Figure 3.2: Top: Total annulus-integrated flux of the CO $v=1-0$ (R7) line for different rings (coloured lines) and the total disk (black line). The stellar flux for a $T_{\text {eff }}=4060 \mathrm{~K}$ and $R=2.55 R_{\odot}$ star is given by the dashed line. The black asterisks mark the total line flux $F_{\text {line }}^{\text {tot }}$ for each annulus divided by 10. Bottom: Normalised CO $v=1-0$ (R7) lines for different rings (coloured lines) and the total disk (black line).

of the observed line profile at the same time. At these large radii the temperatures are smaller than in the regions closer to the the central star and hence the flux contribution from the disk in the wavelength region observed is greatly reduced. Therefore, a large inclination as proposed by SD08 cannot be confirmed by the models calculated for this analysis. Hence, the further search for a best model was limited to the stellar parameters of BR07.

\subsubsection{Model properties}

Figure 3.2 shows the behaviour of the $\mathrm{CO}$ lines with disk radius $R$ using the $\mathrm{CO} v=1-0$ (R7) line as an example. The lower panel shows normalised line profiles. The line strength, i.e. the ratio of $F_{\nu} / F_{\text {cont }}$, increases with increasing disk ring radius $R$ up to $R=0.200$ AU. At larger radii the line disappears due to a sudden drop of the CO abundance given by ACES. The upper panel shows the total annulus integrated flux $F_{\nu}^{\text {ring }}=\int_{R_{\text {in }}}^{R_{\text {out }}} F_{\nu}(R) d R$ for disk annuli with different radii $R$ (coloured line profiles). The total disk integrated flux is shown as solid black line and the flux contribution of the central star to the CO line is denoted by the dashed black line. It is evident that despite the small surface fractions the inner disk rings provide most of the flux in the 


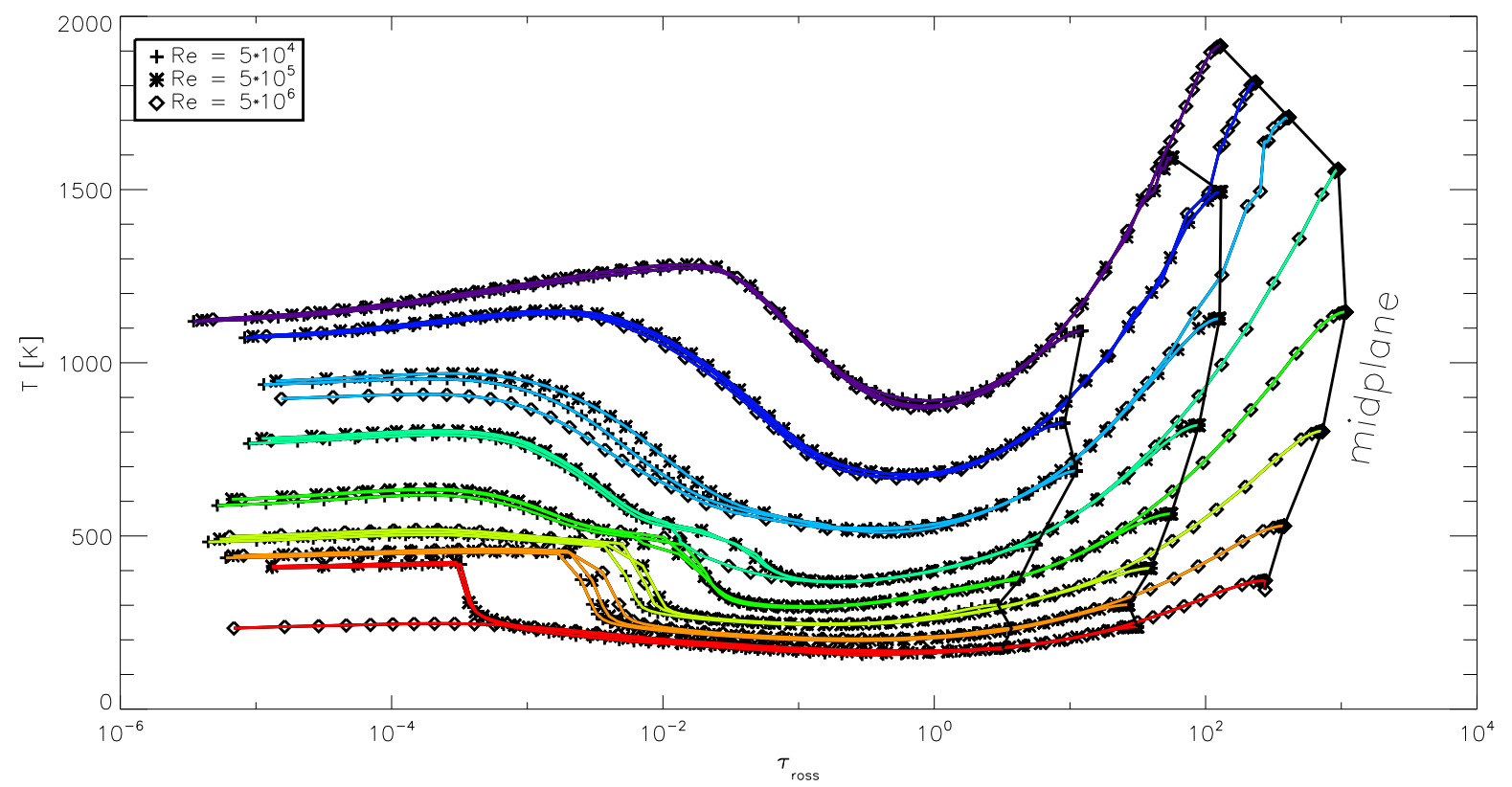

Figure 3.3: Model temperature structures for different Reynolds numbers (coloured lines). The structures correspond in colour to the radii $R$ given in Fig. 3.2. The midplane layers are marked by solid black lines.

wavelength region observed because they are warmer than the outer annuli. In principle, the outer two annuli with $R=0.290 \mathrm{AU}$ and $R=0.422 \mathrm{AU}$ can be neglected because they do not contribute to the integrated disk spectrum perceptibly. This ensures that for the observed wavelength range the fraction of the disk considered is sufficiently large. Furthermore, the asterisks in the upper panel show the continuum-subtracted and wavelength- and annulus-integrated line profile fluxes $F_{\text {line }}(R)$ for the different rings. The run of $F_{\text {line }}(R)$ visualises that the inner rings contribute most to the total line flux $F_{\text {line }}^{\text {tot }}$ despite the increase of line strength in the normalised line profiles with increasing $R$ (lower panel in Fig. 3.2).

\subsubsection{Model vs. observation}

A DISK model with $\dot{M}=3 \times 10^{-9} M_{\odot} \mathrm{yr}^{-1}, R e=5 \times 10^{4}$ corresponding to $\alpha \approx 0.05, R_{\text {in }}=$ $0.052 \mathrm{AU}$, and $R_{\text {out }}=0.500 \mathrm{AU}$ fits the observed spectrum the best when an inclination of $i=22^{\circ}$ is assumed. A comparison between observation and model is shown in Figs. 3.6] and 3.7. How an increase in Reynolds number changes the spectrum when keeping all other parameters constant is displayed in Fig. 3.4. Even though the $R e=5 \times 10^{4}$ model has a small column mass density $\Sigma$, i.e. the disk does not or just barely (in the inner parts) become optically thick in the continuum, the line is less strong than for the models with larger Reynolds number. In Sect. 2.11.2 it is shown that a higher continuum flux level (larger Reynolds number) yields less strong normalised lines. In this particular case, however, the increase in normalised line strength is driven by the CO line in the disk annulus with $R=0.290 \mathrm{AU}$. This annulus does not show CO emission in the $R e=5 \times 10^{4}$ model (see Fig. 3.2). Therefore, a more optically thick model with a larger Reynolds number, as e.g. suggested by the minimum mass solar nebula, predicts too strong CO emission lines. The temperature structures of models with different radii and Reynolds numbers 


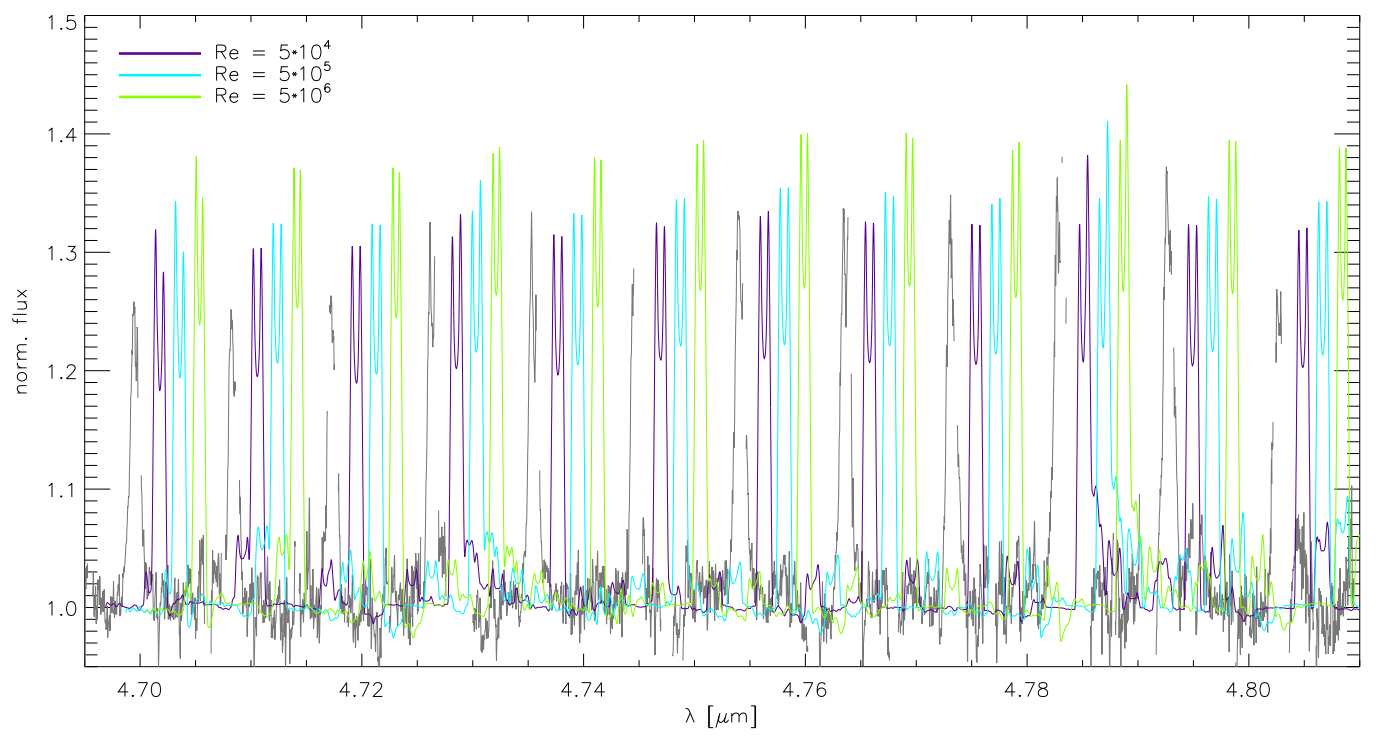

Figure 3.4: Model spectra with different Reynolds numbers (coloured lines) compared to the observed spectrum of GQ Lup (grey line). The increase in Reynolds number yields stronger $\mathrm{CO}$ emission lines.

are shown in Fig. 3.3 .

A detailed comparison between model and observation in Figs. 3.6 and 3.7 reveals that the line wings of the fundamental transition lines $(v=1-0)$ close to the continuum are somewhat broader in the observation than in the model. This argues for contributions of disk regions with high Kepler velocities, i.e. smaller radii $R$. However, if disk rings with smaller radii are included, the temperature sensitive $v=2-1 \mathrm{CO}$ emission lines are too strong in the model. Furthermore, the low $J$ R- and P-branch $\mathrm{CO} v=1-0$ lines around $4.66 \mu \mathrm{m}$ are overestimated by the model, or put in other words, the increment of the line strengths is too strong in the model. For the best model fit, a model which fits the strength of the higher order CO $v=1-0$ lines is used because these are more frequent in the observation accepting a less well reproduction of the low-order lines.

As shown above, the part of the disk with radii $R>0.500$ AU has no measurable influence on the spectrum at wavelength $<5 \mu \mathrm{m}$. The strong $\mathrm{CO}$ fundamental transition lines $(v=1-0)$ in the spectrum are well reproduced by the model. The weaker $v=2-1 \mathrm{CO}$ emission lines are also visible in the spectrum and fitted by the model. The ${ }^{13} \mathrm{CO}$ isotope is weakly present in the model, which assumes the solar system carbon isotope ratio of 89:1, but clear evidence for its existence in the observed spectrum cannot be found. Woods \& Willacv (2009) have modelled isotopic fractionation for a protoplanetary disk quite similar to the GQ Lup model, but found only mild effects on carbon monoxide, with very modest increases in the ${ }^{12} \mathrm{CO} /{ }^{13} \mathrm{CO}$ ratio only in the outermost layers of the disk. Considering the noise in the spectrum and the blending of ${ }^{12} \mathrm{CO}$ and ${ }^{13} \mathrm{CO}$ lines, evidence for a different isotope ratio in the observations cannot be found. The maximum temperature in the surface layers of the disk atmospheres is $T=950 \mathrm{~K}$ which is much smaller than the estimated excitation temperature of $T_{\mathrm{ex}}=1814 \mathrm{~K}$ found by Naiita et al. (2003) for DF Tau which has a similar spectrum but stronger $v=2-1$ emission lines.

In Fig. 3.5 the influence of the dust grain size distribution on the spectrum is shown. All of 


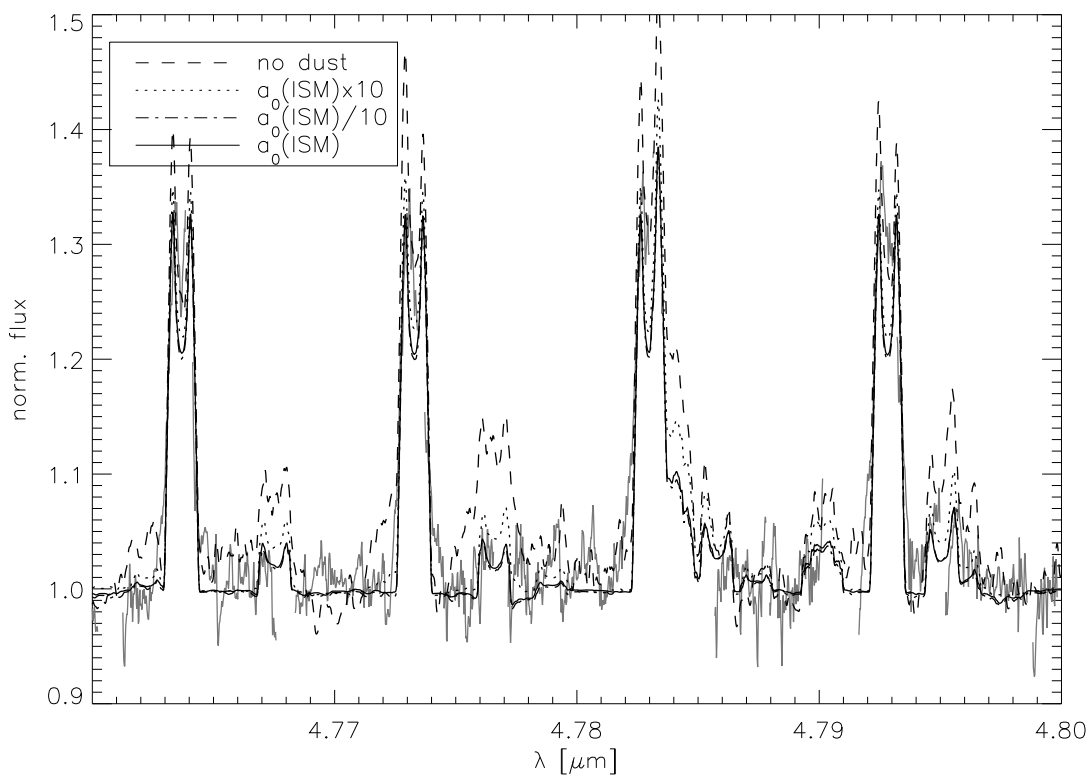

Figure 3.5: Spectra calculated from the same structure model but for different dust grain sizes. Neglecting dust as opacity source results in overestimation of the lines. ISM grain size distribution and a dust grain size setup with ten times smaller dust radii yield a very similar spectrum. The dust opacity is slightly reduced if the grain size is increased by a factor of ten.

the spectra are based on the same structure model but the emergent flux is calculated for four different dust grain size setups, i.e. no dust, ISM grain size distribution with $a_{0}=6.25 \times 10^{-3} \mu \mathrm{m}$, $a_{0}=6.25 \times 10^{-2} \mu \mathrm{m}$, and $a_{0}=6.25 \times 10^{-4} \mu \mathrm{m}$. Neglecting dust leads to a lack of opacity and too strong lines. The ISM grain size distribution fits the data equally well as the smaller grains. The spectrum with ten times larger grains than ISM still reproduces the observation reasonably well, but lines are slightly stronger than for ISM sizes.

An interesting feature in the observed spectrum is the hydrogen $\operatorname{Pf} \beta(7-5)$ emission line. This line has been partially attributed to an absorption line in the telluric standard star used for the calibration of CTTS by Naiita et al. (2003). Since in this analysis a telluric model was used to remove telluric absorption lines, the $\operatorname{Pf} \beta$ feature can unambiguously be related to an emission line intrinsic to GQ Lup. A blue shift of $7 \mathrm{~km} \mathrm{~s}^{-1}$ for the line relative to the star is measured and the width corresponds to a velocity of $u \approx 172 \mathrm{~km} \mathrm{~s}^{-1}$. This value is much larger than the measured $v \sin i=6.8 \mathrm{~km} \mathrm{~s}^{-1}$ by BR07. Therefore, the line cannot be attributed to the star directly. If Keplerian rotation is assumed, the formal origin of the line is at $R=0.025 \mathrm{AU}=2 R_{\star}$ and most probably originates from the accretion flow onto the star. The line width is typical for $\mathrm{H} \mathrm{I}$ recombination lines in accreting T Tauri stars (Salvk et al., 2009).

Finally, the corotation radius gives a consistency check of whether or not the derived inner gas disk radius is correct and the two radii can be compared to literature values. The corotation radius gives the distance from the star where matter rotating at Keplerian velocity has the same angular velocity $\Omega$ as the star. The corotation radius $R_{\mathrm{cr}}$ is believed to be the point where a stellar magnetic field penetrates and truncates the disk and can be calculated by

$$
R_{\mathrm{cr}}=\left(\frac{G M_{\star}}{\Omega^{2}}\right)^{1 / 3} .
$$




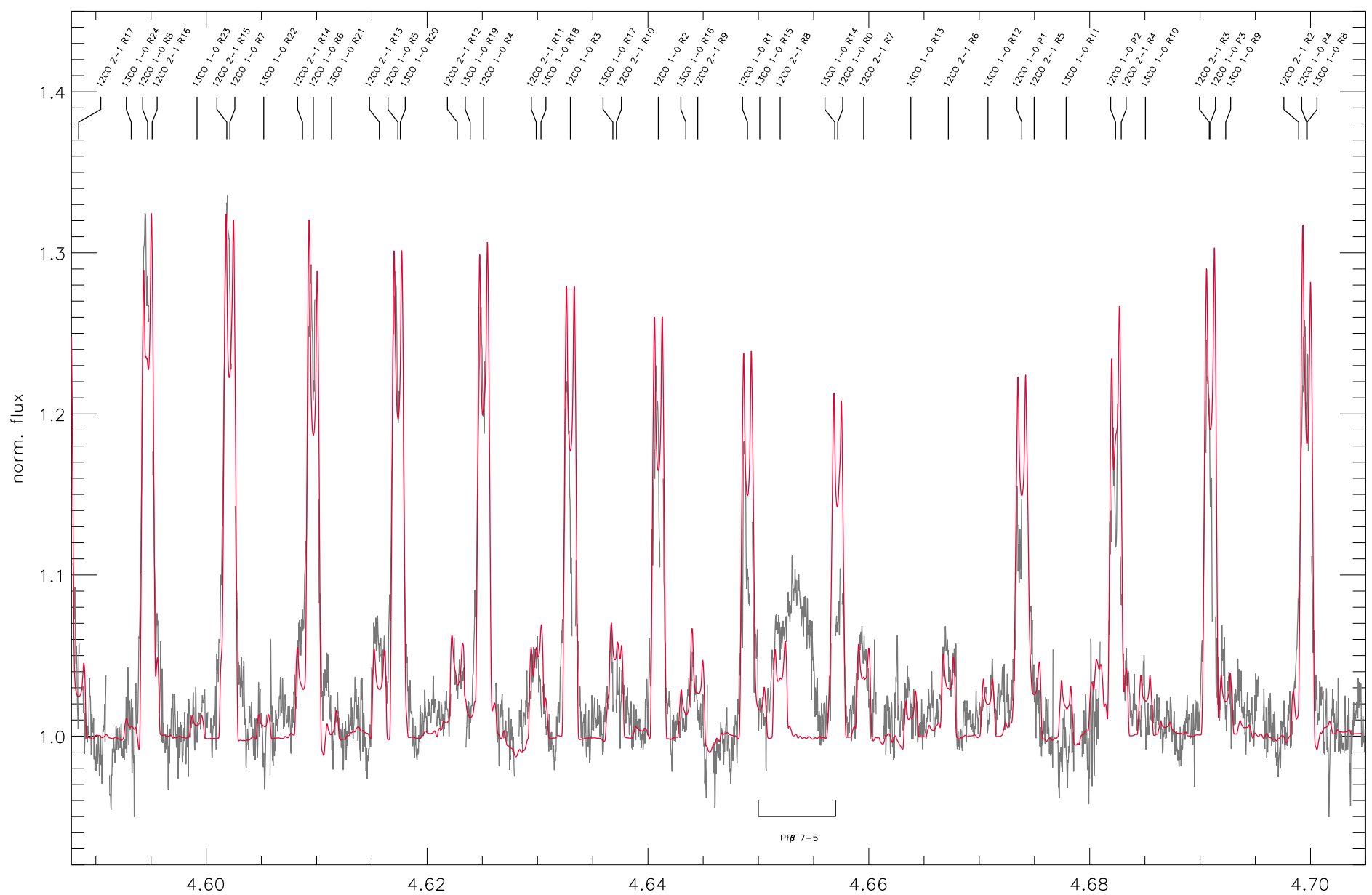

Figure 3.6: CRIRES spectrum of GQ Lup (grey line) with the best-fit disk model (red line). The model is calculated for a disk between $R_{\text {in }}=0.052 \mathrm{AU}$ and $R_{\text {out }}=0.500 \mathrm{AU}$ with a mass accretion rate of $\dot{M}=3 \times 10^{-9} M_{\odot} \mathrm{yr}^{-1}$ and a Reynolds number of $R e=5 \times 10^{4}$. The stellar parameters are those of Broeg et al. $(2007): R_{\star}=2.55 R_{\odot}$ and $T_{\text {eff }}=4060 \mathrm{~K}$. For the stellar mass we adopted the value of $M_{\star}=0.8 M_{\odot}$ given by Seperuelo Duarte et al. (2008). The hydrogen $\operatorname{Pf} \beta(7-5)$ line at $4.654 \mu \mathrm{m}$ cannot be attributed to the disk because its Doppler width corresponds to a Kepler velocity of $R=0.025 \mathrm{AU}=2 R_{\star}$ which lies well within the determined inner disk radius. 


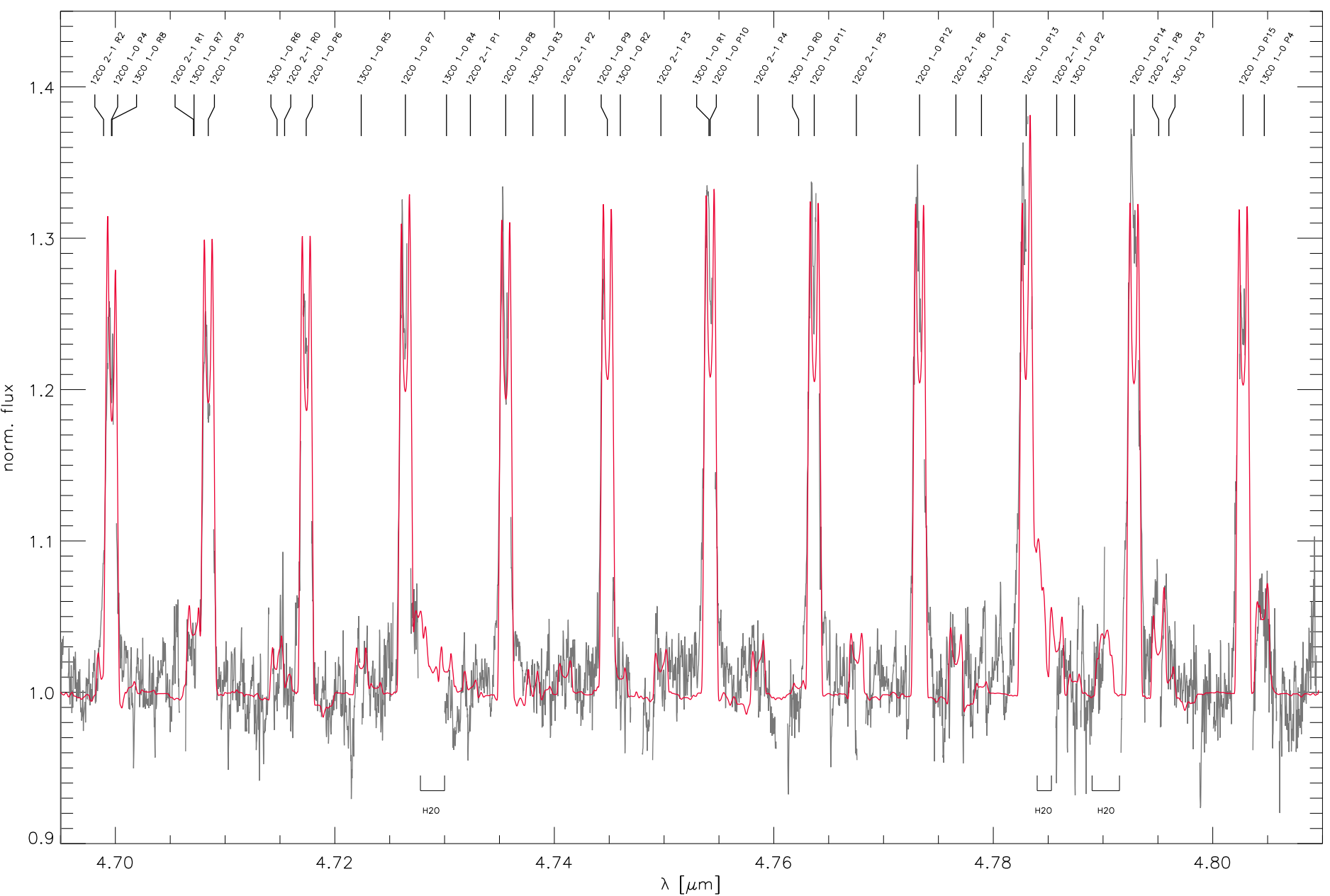

Figure 3.7: CRIRES spectrum of GQ Lup (grey line) with the best-fit disk model (red line). Disk model parameters as in Fig. [3.6] 
For the GQ Lup values given by BR07 the corotation radius is $R_{\mathrm{cr}}=0.067 \mathrm{AU}$ which is slightly larger than the inner gas radius in the best-fit model $R_{\mathrm{in}}=0.052 \mathrm{AU}$. Carr (2007) give an average ratio of the inner gas to the corotation radii of $\sim 0.70$ for a sample of 12 stars. The ratio for the GQ Lup system is 0.77 which is slightly larger but easily within the error of the average value. Hence, the gas in most CTTS reaches within the corotation radius of the star.

\subsection{TW Hya}

\subsubsection{System parameters}

TW Hya is one of the most studied CTTS. It is a rather old YSO with an age of $7-15 \mathrm{Myr}$ (Thi et al., 2004) and it shows a flux deficit in the SED below $10 \mu \mathrm{m}$ which can be assigned to a hole of optically thick dust inwards of $4 \mathrm{AU}$ from the star (Calvet et al., 2002). Observations of the $10 \mu \mathrm{m}$ silicate feature (Sitko et al., 2000) and a non-zero excess at $2 \mu \mathrm{m}$ (Johns-Krull \& Valenti, 2001) points to a remain of some residual dust with sizes less than a few microns within $R=4 \mathrm{AU}$. Furthermore, warm gas has been detected (Herczeg et al., 2004; Rettig et al., 2004; Salvk et al., 2007) which confirms that the inner part of the disk is not devoid of material.

The dust temperature at $R=4 \mathrm{AU}$ is $\sim 100 \mathrm{~K}$ which is far below the sublimation temperature for silicate grains of $\gtrsim 1500 \mathrm{~K}$ (Pollack et al., 1994). Therefore, a different mechanism must cause the clearing of optically thick dust. A hole or gap in circumstellar disks is often assigned to disk clearing caused by a planet which inhibits mass accretion outside its orbit while the material inside can spiral onto the star freely. A viscous disk outside the orbit will cause the planet and the gap to migrate inwards. Hence, a planet is unlikely to preserve the clearing during the disk lifetime because the inner hole is filled on the viscous timescale. Another possible explanation for a disk hole is grain growth which leads to a depletion of small grains which are the main opacity source and hence the origin of near-IR emission. Observations of scattered light and longwavelength emission from the outer $R>10 \mathrm{AU}$ disk region of TW Hya indicate grain growth up to $\mathrm{cm}$ size (Weinberger et al., 2002; Wilner et al., 2005). The small fraction of dust observed within $R=4 \mathrm{AU}$ can be attributed to the tail of a grain size distribution which has its maximum at grain radii above $1 \mu \mathrm{m}$.

Setiawan et al. (2008) report the finding of a close-in planet around TW Hya at $R=0.04 \mathrm{AU}$ distance by the radial velocity technique. Huélamo et al. (2008), however, claim that the changes in radial velocity are rather caused by stellar spots than by a planet. The star is of particular interest for stellar evolution of young objects because it still shows features typical of accretion (broad $\mathrm{H} \alpha$ line and Li I $\lambda 6708$; Muzerolle et al., 2000) despite its age. As for most young objects, the determination of stellar parameters is ambiguous due to stellar activity. The spectral type of $\mathrm{TW}$ Hya is $\mathrm{K} 8 \mathrm{~V}$ which corresponds to an effective temperature of $\sim 4000 \mathrm{~K}$ (interpolated from values given by Kenvon \& Hartmann, 1995). A stellar radius of $R_{\star} \approx 1.05 R_{\odot}$ can be derived using the luminosity of $\log \left(L / L_{\odot}\right)=-0.6$ given by Thi et al. (2004). A mass of $M_{\star}=0.7 \pm 0.1 M_{\odot}$ is listed by Setiawan et al. (2008). Different values for the mass accretion rate are given in the literature. Muzerolle et al. (2000) used magnetospheric accretion models to fit $\mathrm{H} \alpha$ line profiles and receive $\dot{M}=5 \times 10^{-10} M_{\odot} \mathrm{yr}^{-1}$. Alencar \& Batalha (2002) present a somewhat higher rate of $\dot{M}=2 \times 10^{-9} M_{\odot} \mathrm{yr}^{-1}$.

The regions in the inner dust hole is filled with optically thin gas which can be observed in the IR. Rettig et al. (2004) and Salvk et al. (2007) have investigated the CO spectrum of TW Hya around $4.7 \mu \mathrm{m}$. From rotational diagrams they determine an LTE temperature of $430 \pm 40 \mathrm{~K}$ and $750_{-200}^{+350} \mathrm{~K}$, respectively. Salvk et al. (2007) calculated inner disk radii of $R_{\text {in }}=0.20_{-0.05}^{+0.40} \mathrm{AU}$ 


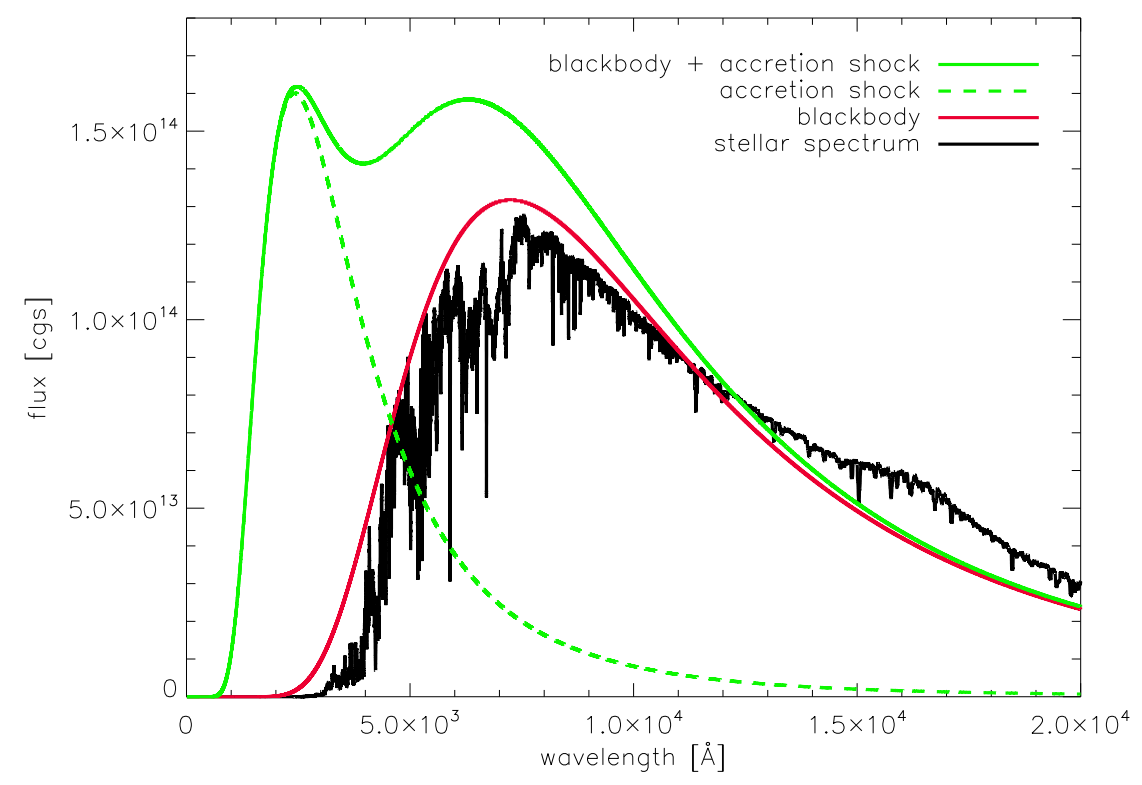

Figure 3.8: Comparison of TW Hya model irradiation sources. The black line is a PHOENIX model with $T_{\text {eff }}=4000 \mathrm{~K}, \log g=4.5$, and $[\mathrm{M} / \mathrm{H}]=0.0$. The red line is a blackbody spectrum of the same effective temperature. The dashed green line is a blackbody of $T_{\text {eff }}=12000 \mathrm{~K}$ which is added to simulate the flux coming from accretion shocks with a filling factor of $f=0.005$. The solid green line is a combination of both blackbodies.

from the inspection of the line profiles and $R_{\text {in }}=0.07_{-0}^{+0.36} \mathrm{AU}$ from the rotational temperature. A newer investigation of the authors (Salvk et al., 2009) yields an inner CO radius of $R_{\mathrm{CO}}=$ $0.10_{-0.04}^{+0.20}$ AU. Rettig et al. (2004) place the inner radius at $R_{\text {in }}=0.50 \mathrm{AU}$ from their rotational temperature. Using interferometry and IR-photometry, Eisner et al. (2006) derive an inner disk radius of $R_{\text {in }}=0.06 \pm 0.01 \mathrm{AU}$ for optically thin dust.

\subsubsection{Model vs. observation}

Since the inner 4 AU of TW Hya's circumstellar disk are partially cleared of material, the column mass densities $\Sigma$ are quite small. Salvk et al. (2009) derive a value of $\Sigma_{n}^{\text {gas }}=7 \times 10^{-2} \mathrm{~g} \mathrm{~cm}^{-2}$ from their $\mathrm{CO}$ excitation diagram using a ratio of $\mathrm{H}_{2} / \mathrm{CO}=5 \times 10^{3}$ (Lacv et al., 1994), which is one of the lowest observed values and should therefore provide a lower limit to $\Sigma_{\text {gas }}$. Eisner et al. (2006) measured $\Sigma_{0}=6.3 \pm 1.5 \times 10^{-7} \mathrm{~g} \mathrm{~cm}^{-2}$ of optically thin dust. Using the relation $\Sigma=$ $\Sigma_{0}(R / \mathrm{AU})^{-3 / 2}$ yields a gas-to-dust ratio that is $\sim 10^{5}$ times higher at $0.2 \mathrm{AU}$ than the standard gas-to-dust ratio of 100 .

As a starting point for the model fits, sets of disk rings were calculated for the same radii as in the case of GQ Lup and upper and lower mass accretion rates of $\dot{M}=2 \times 10^{-9} M_{\odot} \mathrm{yr}^{-1}$ and $\dot{M}=5 \times 10^{-10} M_{\odot} \mathrm{yr}^{-1}$ (see previous Section) were chosen. Reynolds numbers of $R e=5 \times 10^{3}$ and $R e=5 \times 10^{4}$ were set to provide small column densities. All combinations of $\dot{M}, R e$, and $R_{\text {in }}$ provided too weak $\mathrm{CO}$ emission lines compared to the observation using an inclination of $i=4.1^{\circ} \pm 1.0^{\circ}$ (Pontoppidan et al., 2008). Hence, an increase in temperature in the outer disk atmosphere layers is needed to generate stronger CO lines (see Sect. 2.11.3). Usually a blackbody 


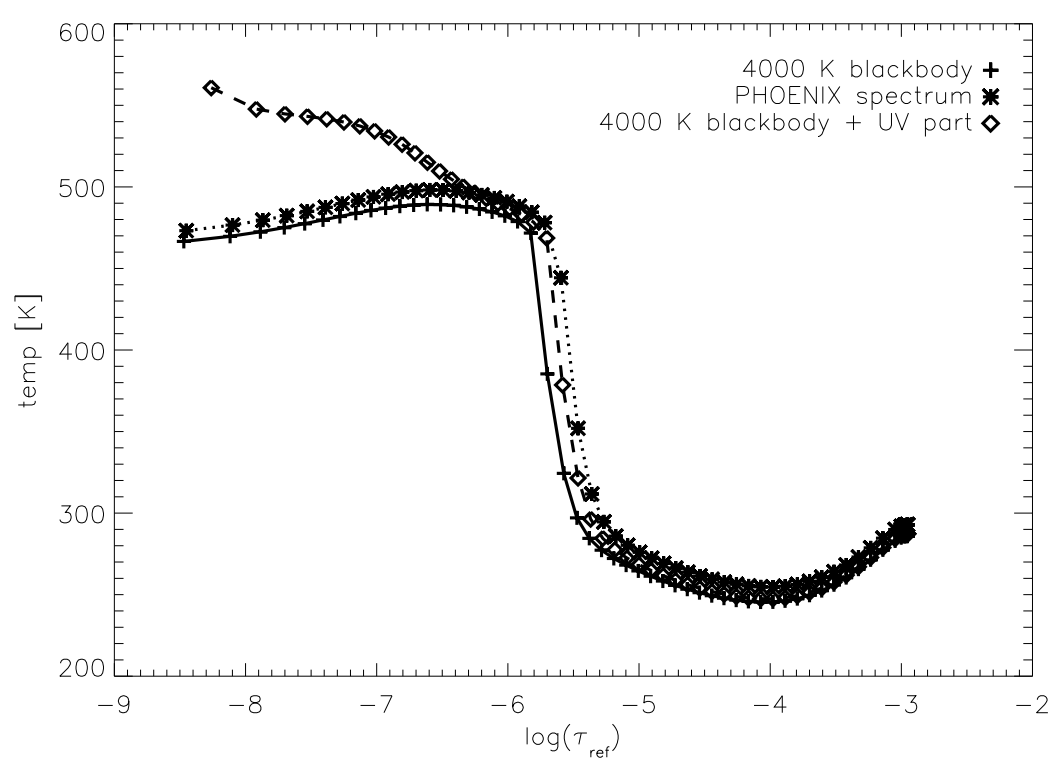

Figure 3.9: Temperature structures for different irradiation source treatments. The crosses mark the temperatures for a disk ring at $R=0.094$ AU which result from a $4000 \mathrm{~K}$ blackbody irradiation source, the asterisks are for a PHOENIX stellar model with $T_{\text {eff }}=4000 \mathrm{~K}, \log g=4.5$, and $[\mathrm{M} / \mathrm{H}]=0.0$, and the diamonds for a blackbody with an additional UV part of $T_{\text {eff }}=15000 \mathrm{~K}$ and a filling factor of $f=0.001$.

of a given stellar effective temperature is used as irradiation source because uncertainties in stellar parameters are usually large and the additional calculation of stellar models for irradiation is time consuming. In the case of TW Hya, the effect of using a stellar model instead of a blackbody of stellar temperature on the disk structure is investigated. In Fig. 3.8 the flux of a PHOENIX model with $T_{\text {eff }}=4000 \mathrm{~K}, \log g=4.5$, and $[\mathrm{M} / \mathrm{H}]=0.0$ is compared to a blackbody of equal effective temperature. Apparently, the stellar model has a stronger flux output at large wavelengths than the blackbody. Since the scattering fraction is smaller at these wavelengths, more radiative energy is converted into thermal energy and the disk becomes hotter (see Fig. 3.9). Despite this increase in temperature, the $\mathrm{CO}$ emission lines still do not become strong enough to fit the observations.

Since TW Hya is known to be actively accreting (e.g. Muzerolle et al., 2000), high energetic radiation is produced in the accretion shocks as matter falls onto the stellar surface via the magnetic field lines. Calvet \& Gullbring (1998) have analysed the structure and emission of this type of shock in $\mathrm{T}$ Tauri stars quantitatively and find the following relation for the flux carried into the shock by the accretion column:

$$
\mathcal{F}=9.8 \times 10^{10} \mathrm{ergs} \mathrm{cm}^{-2} \mathrm{~s}^{-1}\left(\frac{\dot{M}}{10^{-8} M_{\odot} \mathrm{yr}^{-1}}\right)\left(\frac{M_{\star}}{0.5 M_{\odot}}\right)\left(\frac{R_{\star}}{2 R_{\odot}}\right)^{-3}\left(\frac{f}{0.01}\right)^{-1} .
$$

Inserting the values for TW Hya of $M_{\star}=0.7 M_{\odot}, R_{\star}=1 R_{\odot}$, a typical filling factor of $f=0.005$, and the mass accretion rate of $\dot{M}=5 \times 10^{-10} M_{\odot} \mathrm{yr}^{-1}$ of Muzerolle et al. (2000) gives a value of $\log \mathcal{F}=11$. Calvet \& Gullbring (1998) have calculated model parameters resulting from the accretion shock. For a star with $M_{\star}=0.8 M_{\odot}, R_{\star}=1.5 R_{\odot}$, and $T_{\text {eff }}=4350 \mathrm{~K}$ they receive for $\log \mathcal{F}=11$ a temperature of $T_{\mathrm{eff}}^{\mathrm{UV}}:=T\left(\tau_{\text {ross }}=2 / 3\right)=6040 \mathrm{~K}$. Even though the radius of TW Hya of $R_{\star}=1 R_{\odot}$ is smaller than the one used to determine the temperature of the emitting 


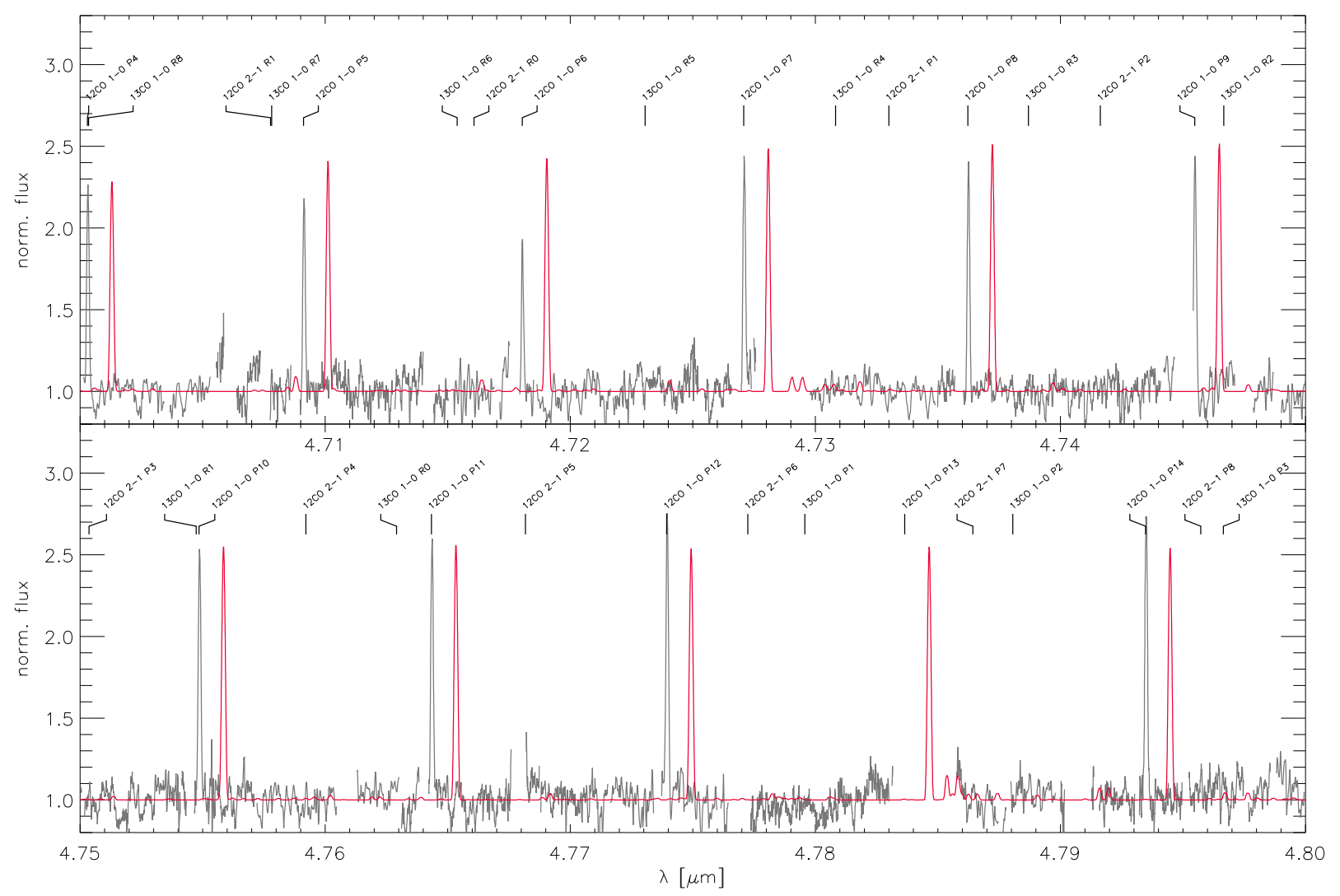

Figure 3.10: CRIRES spectrum of TW Hya (grey line) with the best-fit disk model (red line). The model has $\dot{M}=2 \times 10^{-9} M_{\odot} \mathrm{yr}^{-1}, R e=5 \times 10^{3}, T_{\text {eff }}^{\mathrm{UV}}=12000 \mathrm{~K}, f=0.005$, and $R_{\text {in }}=0.090 \mathrm{AU}$. The model is offset in wavelength for clarity.

shock region, and therefore the temperature value $T_{\text {eff }}^{\mathrm{UV}}$ for a $\log \mathcal{F}=11 \mathrm{TW}$ Hya will be higher, it is not heating the disk enough to produce $\mathrm{CO}$ emission lines of the strength of the observation.

For the higher mass accretion rate of $\dot{M}=2 \times 10^{-9} M_{\odot} \mathrm{yr}^{-1}$ given by Alencar \& Batalha (2002) the flux is $\log \mathcal{F}=11.7$. Although the value for $T_{\text {eff }}^{\mathrm{UV}}$ is hard to predict from $\log \mathcal{F}$ and since the results listed in Calvet \& Gullbring (1998) are very limited in parameter space, a combination of $T_{\mathrm{eff}}^{\mathrm{UV}}=12000 \mathrm{~K}$ and $f=0.005$ provides a good fit to the data assuming an inclination of $i=3.1^{\circ}$, which is the lower limit of the value given by Pontoppidan et al. (2008), and an inner disk radius of $R_{\text {in }}=0.090 \mathrm{AU}$ (see Fig. [3.10). The inner disk radius lies well within the values given by Salyk et al. (2009) and Eisner et al. (2006). A larger inner model radius would result in narrower emission lines which would fit the observations even better and narrower lines would permit larger inclinations as given in the literature. However, one should not forget that the line width is also a function of the assumed statistical velocity which is set to a fixed value of $2 \mathrm{~km} \mathrm{~s}^{-1}$. Salvk et al. (2009) derive an inner CO radius $R_{\mathrm{CO}}$ from CO line profile composites using a simple disk model using a power law temperature given by $T=300 \mathrm{~K}(R / \mathrm{AU})^{-\alpha}$ with $\alpha=0.4$ for dust in a passive disk of submicron-sized grains (Chiang \& Goldreich, 1997). The inner radius $R_{\text {in }}$ is varied until the emission line profile fits the line best. This simple disk model assumes optically thin lines and an emission region of vertical uniform temperature. In Fig. 3.11 the temperature in the outer layers of the best-fit DISK model is compared to the power law temperature of Salvk et al. (2009). Clearly, the temperatures assumed by the latter authors are significantly larger than the 

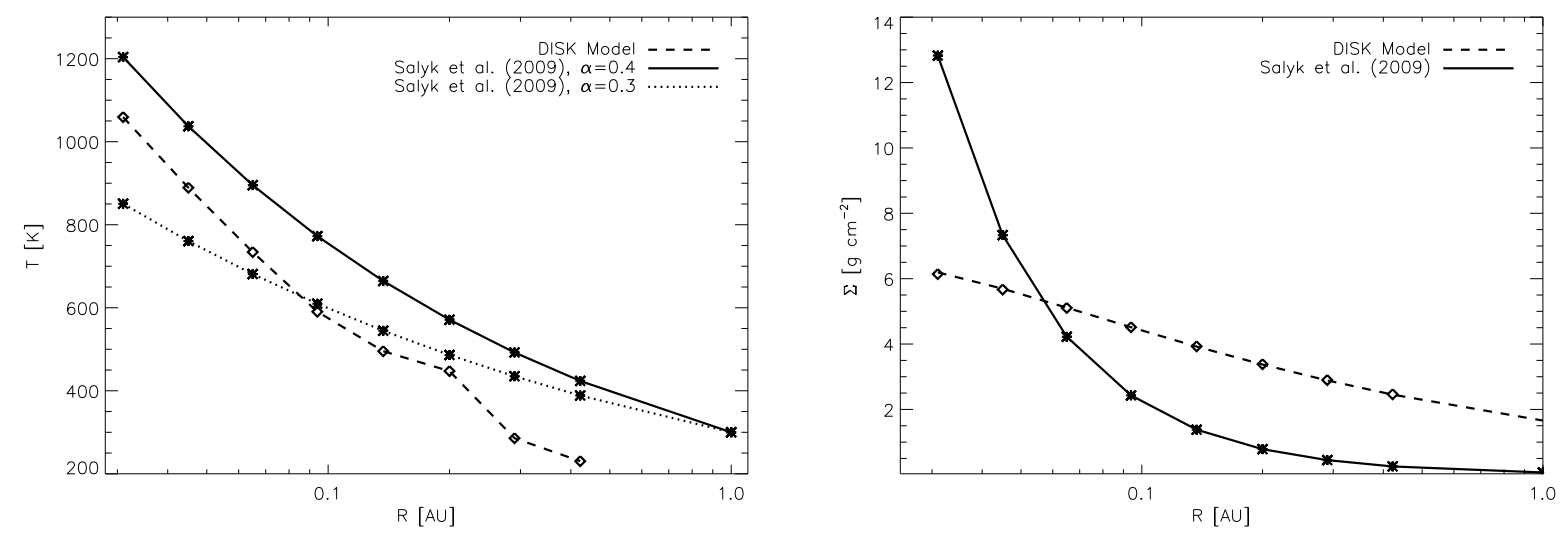

Figure 3.11: Temperature $T$ (left plot) and surface density $\Sigma$ (right plot) over radius $R$ for DISK model (dashed line) and power law description for the values of Salvk et al. (2009) (solid/dotted line).

ones resulting from the self-consistent 1D disk structure computations presented in this work. An exponent of $\alpha=0.3$ agrees better with the DISK model data. Salvk et al. (2009) also assumed that $\mathrm{CO}$ is continuously present in the surface layers of the disk. The equation of state used for the DISK models, however, shows a sensitive dependency of the CO partial pressure on temperature and density which leads to the disappearance of the emission lines beyond $R \approx 0.200$ AU.

Even though a model with $R e=5 \times 10^{4}$ provides an equally good fit to the data, the model with $R e=5 \times 10^{3}$ has been selected here because it yields the smaller column density $\Sigma$. This result is in better agreement with the work of Salvk et al. (2009) and Eisner et al. (2006) who both report small densities in the inner part of the TW Hya disk. A comparison between the model surface density $\Sigma \propto\left(\frac{1}{\sqrt{R}}-\frac{\sqrt{R_{\star}}}{R}\right)$ (see Eq. (2.4) ) and the one given by $\Sigma=7 \times 10^{-2} \mathrm{~g} \mathrm{~cm}^{-2}(R / \mathrm{AU})^{-3 / 2}$ (Salvk et al., 2009) are shown in Fig. 3.11. The two functions $\Sigma(R)$ behave quite differently. In the regime of small $R$ values, the power law relation rises very steeply while the $\Sigma$ values used for the DISK models are smoother but lead to much higher surface densities at $R=1$ AU.

The comparison between power law and self-consistent DISK model surface density or temperature run with radius shows significant differences. The ad-hoc models used by e.g. Salvk et al. (2009) or Eisner et al. (2006) yields a steep drop-off in surface density leading to very large values at small $R$ or very small values $\Sigma(R=1 \mathrm{AU})$, respectively. The temperatures are larger than the ones derived by the DISK code even though the calculations with the latter already assume a significant amount of high energetic radiation which powers the rise in temperature in the outer layers. Due to these various differences in the model assumptions, it appears that one needs to be very careful in interpreting the results.

\section{$3.3 \quad$ RNO 90}

\subsubsection{System parameters}

RNO 90 is a less well studied object which is one of only two pre-main sequence stars in the small dark cloud L43 - the other being RNO 91. Lahuis et al. (2007) list RNO 90 as a 6 Myr old G5 star for which Kenvon \& Hartmann (1995) give an effective temperature of $T_{\text {eff }}=5770 \mathrm{~K}$. Assuming a metallicity of $Z=0.02$, a luminosity of $L=9 L_{\odot}$ is needed for a G5 star of given 


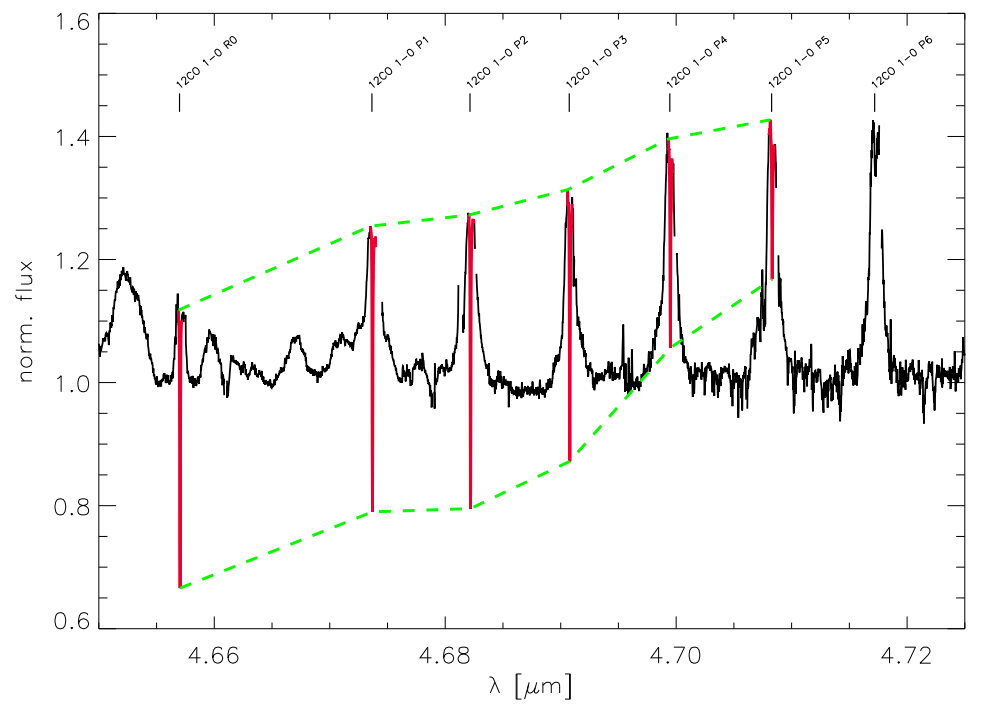

Figure 3.12: A part of the CRIRES CO emission spectrum (black line) with thin $\mathrm{CO}$ absorption features (red lines). The green dashed lines mark the maximum $I_{0}$ and minimum flux $I$ of the thin absorption lines.

age and effective temperature (Siess et al., 2000). The mass and radius then yield $M_{\star}=1.9 M_{\odot}$ and $R_{\star}=2.8 R_{\odot}$. Neither mass accretion rates nor surface densities (which is proportional to the Reynolds number) for RNO 90 are given in the literature. Therefore, these parameters need to be varied to find a best-fit model.

Weintraub et al. (1994) observed both objects with a low resolution IR spectrograph in the wavelength range between $3-5 \mu \mathrm{m}$. They detect signatures which they attribute to frozen water and carbon monoxide on the surface of dust grains along the line of sight to RNO 91. For RNO 90 such detections cannot be confirmed and hence the frozen material is believed to be intrinsic to a circumstellar disk around RNO 91 rather than intercloud material. A part of the CRIRES spectrum of the $\sim 6$ Myr old G5 star is shown in Fig. 3.12. Clearly, the $\mathrm{R}(0)$ and the $\mathrm{P}(1)-\mathrm{P}(5)$ broad double-peaked CO emission lines, which most likely originate in the inner circumstellar gas disk of RNO 90, show narrow absorption features. Because the lines are so narrow a high velocity component in the direction of the observer of the absorbing $\mathrm{CO}$ can be excluded.

A simple estimate of the amount of absorbing material can be made by setting

$$
I_{\nu}=I_{0} \exp \left(-\tau_{\nu}\right)
$$

where $I_{0}$ is the intensity entering the absorption region and $I_{\nu}$ is the intensity leaving the cloud which can be measured from the observations (see Fig. 3.12). The optical depth $\tau_{\nu}$ can be rewritten as

$$
\tau_{\nu}=\int_{0}^{\infty} \kappa_{\nu}(z) d z=\frac{A_{\mathrm{ul}} c^{2}}{8 \pi \nu^{2}} \frac{g_{\mathrm{u}}}{g_{\mathrm{l}}} \phi_{\nu} \int_{0}^{\infty} n_{\mathrm{l}}(z) d z \quad,
$$

where the integral has the dimensions $\left[\mathrm{cm}^{-2}\right]$ and can thus be interpreted as a $\mathrm{CO}$ column density along the line of sight. Table 3.2 and Fig. 3.13 show the fitting results to the ${ }^{12} \mathrm{CO}$ and ${ }^{13} \mathrm{CO}$ $v=1-0$ absorption lines. Since the ${ }^{12} \mathrm{CO}$ absorption lines are superimposed onto the broad double-peaked emission lines a continuum intensity level $I_{0}$ is not unambiguously identifiable. However, the magnitude of absorptive material is not influenced by the precise determination of 
Table 3.2: ${ }^{12} \mathrm{CO}$ and ${ }^{13} \mathrm{CO} v=1-0$ absorption line parameters for RNO 90. For each line that shows CO absorption the optical depth $\tau$, the Einstein A-value $A_{\mathrm{ul}}$, as well as the computed $\mathrm{CO}$ column density $\Sigma_{\mathrm{CO}}$ are given.

\begin{tabular}{|c|c|c|c|c|c|c|c|}
\hline \multicolumn{4}{|c|}{${ }^{12} \mathrm{CO}$} & \multicolumn{4}{|c|}{${ }^{13} \mathrm{CO}$} \\
\hline line & $\tau$ & $\begin{array}{c}A_{\mathrm{ul}} \\
{\left[\mathrm{s}^{-1}\right]}\end{array}$ & $\begin{array}{c}\Sigma_{\mathrm{CO}} \times 10^{15} \\
{\left[\mathrm{~cm}^{-2}\right]}\end{array}$ & line & $\tau$ & $\begin{array}{c}A_{\mathrm{ul}} \\
{\left[\mathrm{s}^{-1}\right]}\end{array}$ & $\begin{array}{c}\Sigma_{\mathrm{CO}} \times 10^{15} \\
{\left[\mathrm{~cm}^{-2}\right]}\end{array}$ \\
\hline $\mathrm{R}(0)$ & 0.499 & 12 & 1.30 & $\mathrm{R}(3)$ & 0.092 & 14 & 0.50 \\
\hline $\mathrm{P}(1)$ & 0.432 & 34 & 3.50 & $\mathrm{R}(2)$ & 0.242 & 14 & 1.10 \\
\hline $\mathrm{P}(2)$ & 0.449 & 23 & 3.20 & $\mathrm{R}(1)$ & 0.237 & 13 & 1.00 \\
\hline $\mathrm{P}(3)$ & 0.359 & 20 & 2.50 & $\mathrm{R}(0)$ & 0.284 & 10 & 0.85 \\
\hline $\mathrm{P}(4)$ & 0.244 & 19 & 1.50 & $\mathrm{P}(1)$ & 0.248 & 31 & 2.15 \\
\hline $\mathrm{P}(5)$ & 0.177 & 18 & 1.00 & $\mathrm{P}(2)$ & 0.213 & 21 & 1.50 \\
\hline Mean & & & 2.17 & Mean & & & 1.18 \\
\hline
\end{tabular}

$I_{0}$. The bigger problem results from the large optical depth of up to $\tau=0.5$ for the absorption lines which makes the ansatz presented in Eq. (3.3) inaccurate. For such large optical depths, radiative transfer effects need to be considered but the thin approach is acceptable for the determination of lower column density limits (especially because there is no other tool such as the more optically thin ${ }^{12} \mathrm{CO} v=2-0$ lines used by Smith et al. (2009) present in the data). The ${ }^{13} \mathrm{CO}$ lines are less optically thick and also do not suffer from superpositions with disk intrinsic emission lines.

From the six line measurements each (see Fig. 3.13), a mean column density of $\Sigma_{12} \mathrm{CO}=$ $2.17 \times 10^{15} \mathrm{~cm}^{-2}$ and $\Sigma_{13} \mathrm{CO}=1.18 \times 10^{15} \mathrm{~cm}^{-2}$ has been derived. The measured ratio of ${ }^{12} \mathrm{CO}:{ }^{13} \mathrm{CO} \approx 2: 1$ strongly deviates from the solar system carbon isotope ratio of $89: 1$ and shows that at least the ${ }^{12} \mathrm{CO} v=1-0$ absorption lines in RNO 90 are not adequate for the calculation of the column density $N$ with the optically thin gas assumption.

\subsubsection{Model vs. observation}

A model grid for a disk system with a central star of $T_{\text {eff }}=5770 \mathrm{~K}, M_{\star}=1.9 M_{\odot}$ and $R_{\star}=2.8 R_{\odot}$ was calculated. The mass accretion rate of the disk was varied between $\dot{M}=10^{-8} M_{\odot} \mathrm{yr}^{-1}$ and $10^{-10} M_{\odot} \mathrm{yr}^{-1}$ with Reynolds numbers of $R e=10^{5}$ and $10^{6}$. Since the central star is assumed to be relatively old for a CTTS the low mass accretion rates are appropriate. Also, high energetic emission from the star is not considered for RNO 90 opposite to the case of TW Hya which is known to be active.

The comparison between a disk model with an inclination of $i=13^{\circ}, \dot{M}=10^{-10} M_{\odot} \mathrm{yr}^{-1}$, $R e=10^{6}, R_{\text {in }}=0.094 \mathrm{AU}$, and $R_{\text {out }}=0.343 \mathrm{AU}$ is shown in Fig. 3.14 A turbulent velocity of $v_{\text {turb }}=2 \mathrm{~km} \mathrm{~s}^{-1}$ is assumed for these calculations (as well as for all previously shown models). This value is higher than the typical thermal velocity in inner disks but this only influences the line profile $\left(\Delta \lambda=3 \times 10^{-5} \mu \mathrm{m}\right.$ at $\left.\lambda=4.7 \mu \mathrm{m}\right)$ and the vertical disk structure very little compared to a model with a pure thermal velocity field. This is especially true when the line profile is additionally broadened by the rotation of the disk. The observed spectrum of RNO 90 shown in Fig. 3.14 has very broad emission line wings compared to the model. This is true for the $v=1-0$ as well as the $v=2-1 \mathrm{CO}$ line. The latter appears to be more triangular in shape than the former and also lacks the characteristic double-peaked shape typical for CTTS. This seems to be contradictory to the canonical interpretation that the $v=2-1 \mathrm{CO}$ lines form in the warmer regions of the disk 

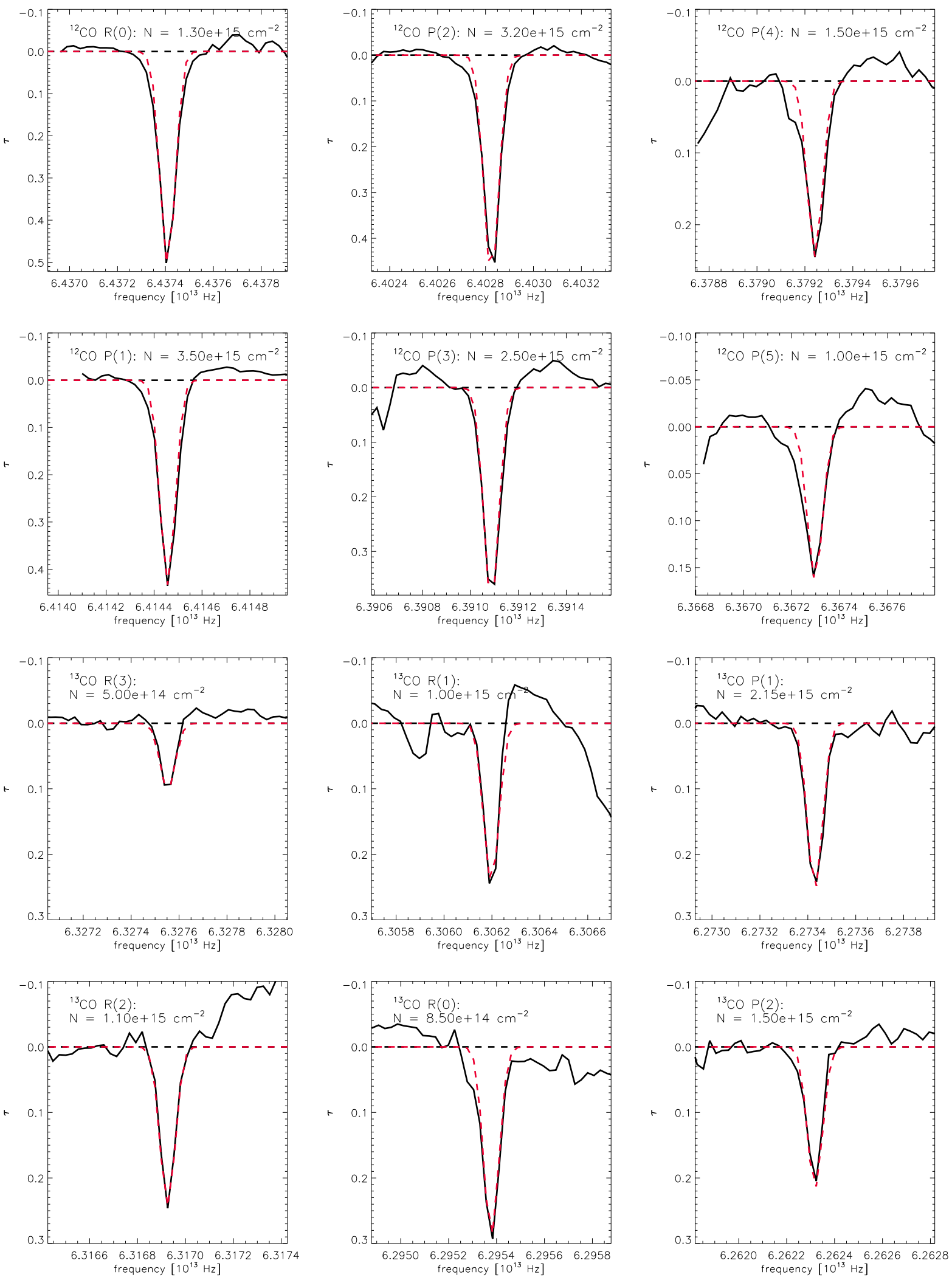

Figure 3.13: ${ }^{12} \mathrm{CO}$ and ${ }^{13} \mathrm{CO}$ absorption line fits for RNO 90. For all lines a FWHM of $\Delta \nu=4.5 \times 10^{8} \mathrm{~Hz}$ is assumed. The estimated column density $N$ is given for each line. 


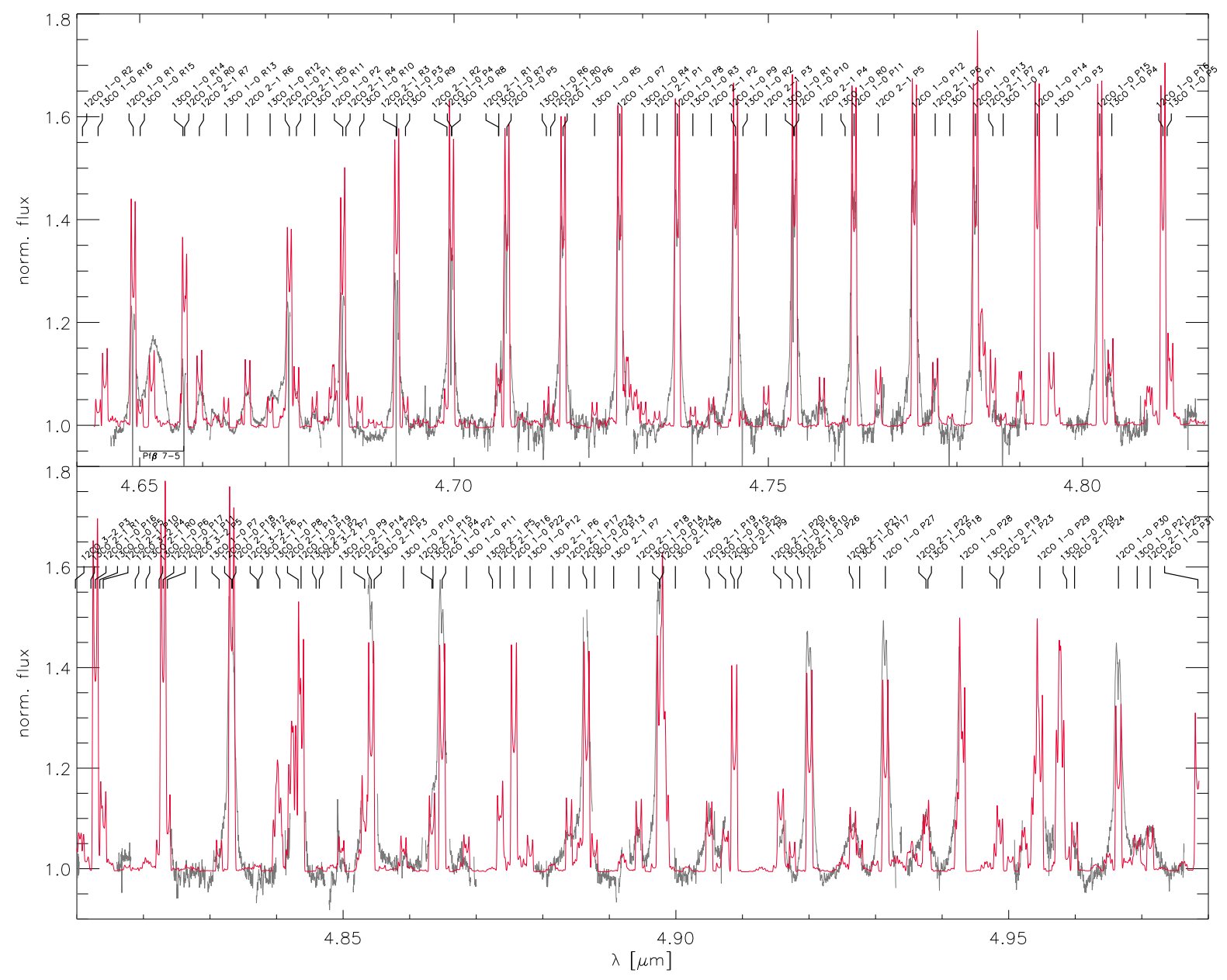

Figure 3.14: CRIRES spectrum of RNO 90 (grey line) compared to a disk model (red line) with $i=13^{\circ}, \dot{M}=10^{-10} M_{\odot} \mathrm{yr}^{-1}, R e=10^{6}, R_{\text {in }}=0.094 \mathrm{AU}$, and $R_{\text {out }}=0.343 \mathrm{AU}$. The assumed model intrinsic turbulent velocity is $v_{\text {turb }}=2 \mathrm{~km} \mathrm{~s}^{-1}$.

at smaller radii than the $v=1-0$ lines and hence are broader and more double-peaked than the latter (Carr, 2007). Furthermore, the $v=1-0$ double-peaked line profiles formed at small radii are "filled" by narrow line contributions forming at larger radii and smaller temperatures. At these low temperatures the $v=2-1$ transitions are not excited anymore. The standard models that fit the CRIRES spectrum of GQ Lup and TW Hya satisfyingly cannot reproduce the broad line profiles observed for RNO 90. Additionally, the double-peaked form of the model line profiles is too pronounced in comparison to the observation (see Fig. 3.14). The width of the line wings can be compensated by including more annuli at smaller radii which however leads to the unintentional effect that the model lines get too strong and also even more double-peaked whereas the opposite is desired.

In order to overcome these obstacles, a stronger turbulent velocity can be the key. Hydrodynamical simulations by Kley and colleagues show that in the upper layers of the disk $(\tau \sim 1)$ the turbulent velocity $v_{\text {turb }}$ is of the order or even larger than the local speed of sound $c_{s}$. One can 


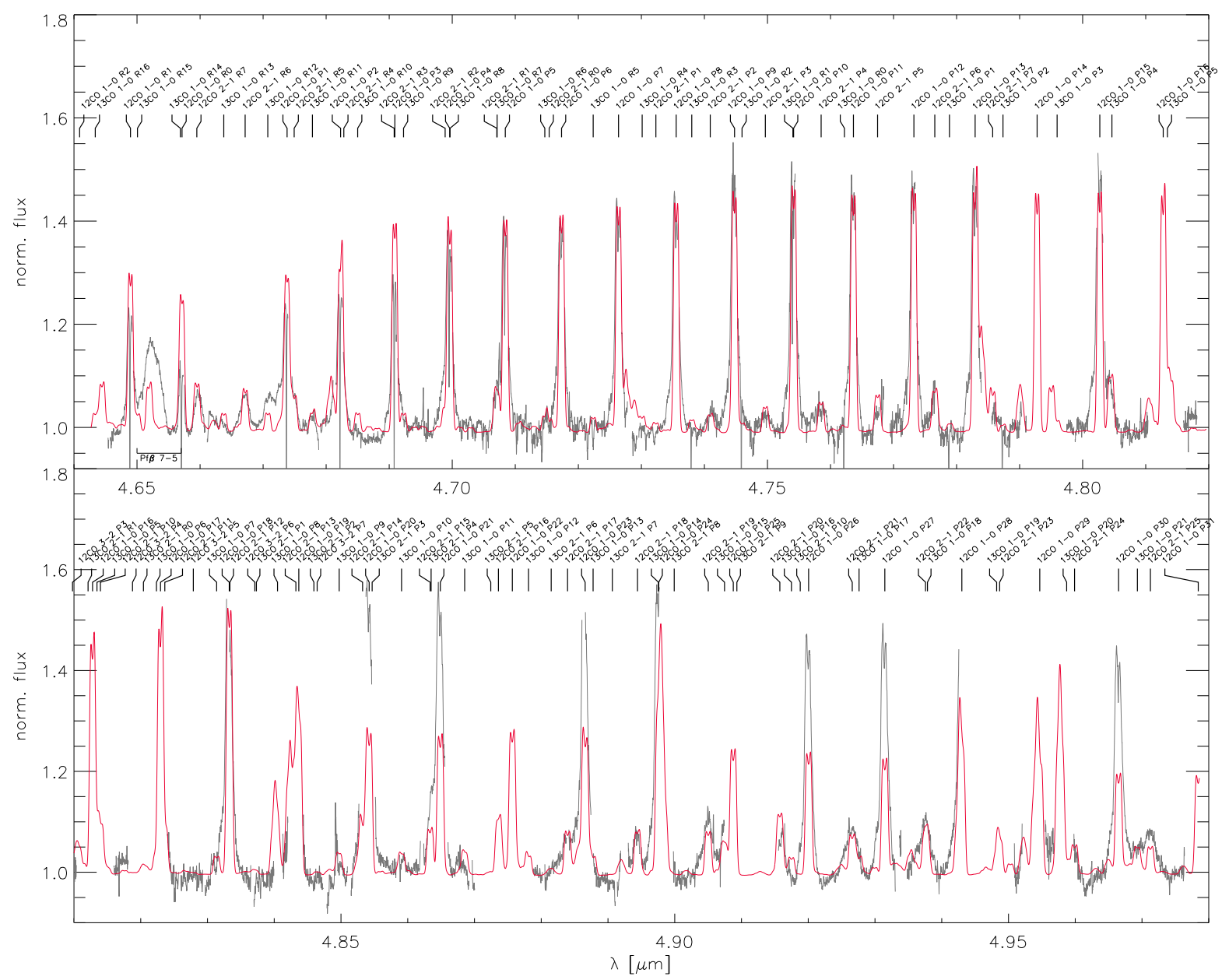

Figure 3.15: CRIRES spectrum of RNO 90 (grey line) with the best-fit disk model (red line). The model parameters are the same as in Fig. [3.14 but the line profile is broadened with a $v_{\text {turb }}=12 \mathrm{~km} \mathrm{~s}^{-1}$ Gaussian.

estimate the value of the latter by

$$
c_{s} \approx u_{\mathrm{Kep}}\left(\frac{H}{R}\right)
$$

where $H$ is the vertical height of the disk at a given radius $R$. Typical values for $H / R$ at inner disk radii in the DISK models are $0.05-0.10$ and the Kepler velocity drops off as $R^{-1 / 2}$. For the RNO 90 central star and an inner disk radius of $R_{\text {in }}=0.094$ AU the turbulent velocity can be expressed by

$$
v_{\mathrm{turb}}=\frac{u_{\mathrm{Kep}}\left(R_{\mathrm{in}}\right)}{\sqrt{R / R_{\mathrm{in}}}} \frac{H}{R} \approx \frac{12 \mathrm{~km} \mathrm{~s}^{-1}}{\sqrt{R / R_{\mathrm{in}}}} .
$$

The synthetic spectrum is then convolved with a Gaussian of $v_{\text {turb }}$ width. With this approach the $v=2-1$ and the $v=1-0 \mathrm{CO}$ lines forming near the inner radius $R_{\text {in }}$ become broader and now fill the line wings in the observed spectrum better (Fig. 3.15) than the model without the additional turbulent broadening (Fig. 3.14). Furthermore, the double-peaked line profile of the 


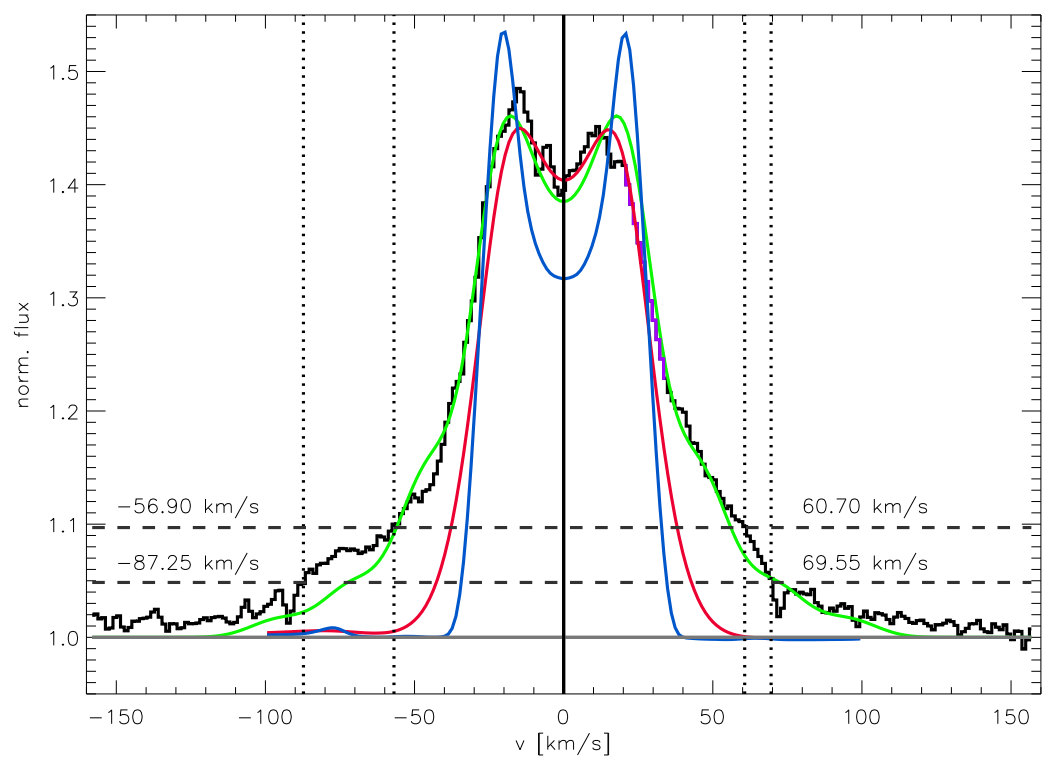

Figure 3.16: Co-added CO emission line profile of RNO 90 from the $\mathrm{P}(6)-\mathrm{P}(13)$ transitions (black line) compared to a DISK model line profile with $v_{\text {turb }}=2 \mathrm{~km} \mathrm{~s}^{-1}$ (blue line), a DISK model line profile with $v_{\text {turb }}=12 \mathrm{~km} \mathrm{~s}^{-1}$ (red line), and a theoretical line profile of Gaussian shape with a width of $v_{\text {turb }}=12 \mathrm{~km} \mathrm{~s}^{-1}$ which was broadened according to the Keplerian rotation of the disk (green line).

$v=2-1$ transition becomes much flatter which is in agreement with the observations. This is also true for the $v=1-0$ transition line which also has some line contribution at larger radii where the turbulent velocity is smaller and the lines are therefore still double-peaked in shape. As in the case of GQ Lup the low-order $v=1-0$ lines are stronger in the model than in the observation. The $v=1-0$ lines of RNO 90 at wavelengths $\lambda>4.84 \mu \mathrm{m}$ are weaker in the model than in the observation while the model $v=2-1$ lines in this spectral region agree quite nicely with the observation. Unfortunately, the spectrum with $\lambda>4.84 \mu \mathrm{m}$ shows some gaps due to telluric absorption which makes a detailed comparison between model and observation difficult.

Figure 3.16 allows a closer look at the RNO 90 line shape of the $\mathrm{CO} v=1-0$ transitions. The black line is a co-added $\mathrm{CO}$ emission line profile from the $\mathrm{P}(6)-\mathrm{P}(13)$ transitions. The summation of individual line profiles reduces the noise level of the observation and partly closes the gaps caused by telluric absorption. The red line wing between $\sim 20-40 \mathrm{~km} \mathrm{~s}^{-1}$ was "filled" using linear interpolation (purple part in Fig. 3.16) because in this region non of the lines hold flux information. In order to investigate the symmetry of the line, the width of the line at $10 \%$ and $20 \%$ (dashed black lines) of the maximal flux level $F_{\max }$ is determined (dotted black lines). The line width at $10 \% F_{\max }$ yields a blue shift in the high-velocity part of the line. At $20 \% F_{\max }$ the line seems to be rather symmetric. Averaging the line width between $1 \%-30 \% F_{\text {max }}$, a mean ratio of $\Delta v_{\text {blue }} / \Delta v_{\text {red }}=1.06$ underlines the impression that the line is slightly amplified in the blue wing. A possible explanation could be a disk wind which is directed towards the observer and adds to the blue wing of the $\mathrm{CO}$ emission line. Alternatively, the blue shifted line flux could originate in the magnetic accretion columns when matter is transferred from the disk to the star. In this case the $\mathrm{CO}$ also moves in vertical direction relative to the disk (at least in the first phase of mass transfer) causing a blue shift of the emission. 
In addition to the line asymmetry, the line wings are very broad pointing towards a highvelocity component which moves towards and away from the observer. In Fig. 3.16 the co-added observed line profile is compared to a DISK CO $v=1-0$ line originating in the model shown in Fig. 3.14 (blue line). While the line width at maximum flux level fits the observation, the line wings are far too narrow: $\Delta v_{\text {mod }}^{10 \%} \approx 70 \mathrm{~km} \mathrm{~s}^{-1}$ versus $\Delta v_{\text {obs }}^{10 \%} \approx 150 \mathrm{~km} \mathrm{~s}^{-1}$. Furthermore, the maximum model flux at $v=0 \mathrm{~km} \mathrm{~s}^{-1}$ and the flux level of the model double peaks cannot be brought into agreement with the observation. Carr et al. (2004) observed the CTTS SVS 13 and found a similar line pattern for the $\mathrm{CO}$ overtone emission as is the case of RNO 90. Their observation could be fit by a model with a non-thermal velocity dispersion of $\sim 11 \mathrm{~km} \mathrm{~s}^{-1}$. The fit to the double peaks in the CRIRES spectra of RNO 90 shown here was significantly improved when the model with the intrinsic $v_{\text {turb }}=2 \mathrm{~km} \mathrm{~s}^{-1}$ is additionally broadened by a Gaussian with $v_{\text {turb }}=12 \mathrm{~km} \mathrm{~s}^{-1}$ (red line) prior to rotational broadening. The width of the line wings is in better agreement with the observation than the $v_{\text {turb }}=2 \mathrm{~km} \mathrm{~s}^{-1}$ model but still lacks some significant flux at $|v|>30 \mathrm{~km} \mathrm{~s}^{-1}$. A theoretical profile that fits the observation well is shown as green line in Fig. 3.16, It is a combination of four Gaussian line profiles with $v_{\text {turb }}=12 \mathrm{~km} \mathrm{~s}^{-1}$ which are broadened according to Keplerian velocities of $u_{\mathrm{Kep}}=444 \mathrm{~km} \mathrm{~s}^{-1}, 333 \mathrm{~km} \mathrm{~s}^{-1}, 222 \mathrm{~km} \mathrm{~s}^{-1}$, and $111 \mathrm{~km} \mathrm{~s}^{-1}$ and an inclination of $i=13^{\circ}$. This corresponds to velocities of $v=100 \mathrm{~km} \mathrm{~s}^{-1}$, $75 \mathrm{~km} \mathrm{~s}^{-1}, 50 \mathrm{~km} \mathrm{~s}^{-1}$, and $25 \mathrm{~km} \mathrm{~s}^{-1}$ if the material is not situated in the disk but rather move directly towards the observer. The individual line contributions are weighted by factors $w=0.1$, $0.2,0.5$, and 1.0 to fit the observations. This composite is not physically motivated but simply serves the demand to fit the line. Actually, the maximum Keplerian velocity of a disk around RNO 90 are reached at the stellar radius are $u_{\mathrm{Kep}}^{\max } \approx 340 \mathrm{~km} \mathrm{~s}^{-1}$. So even if the disk around RNO 90 reached all the way to the surface of the star, the Kepler velocity of $u_{\mathrm{Kep}}=444 \mathrm{~km} \mathrm{~s}^{-1}$ used in the fit (green line in Fig. 3.16) could not be reached. Furthermore, the disk models show that smaller inner radii $R_{\text {in }}$ lead to way too strong lines which do not fit the observations.

If the line was not centrally peaked, one could argue that the broad line wings originate at the given inner radius of $R_{\text {in }}=0.094 \mathrm{AU}$ but for a disk with a larger inclination and that the central part of the line is filled by contributions at large radii (Carr, 2007). Since the inner parts of the disk are much more luminous than the outer ones, a huge line emitting region at larger radii would be needed to fill the central part of the line. However, the DISK models show no $\mathrm{CO}$ line emission at radii $R>0.300 \mathrm{AU}$, an outer $\mathrm{CO}$ radius which is too small to fill the line center for an inclination significantly larger than $i=13^{\circ}$. A disk wind with a maximum escape velocity of the order of the Keplerian velocity $v_{\mathrm{esc}}(R=0.094 \mathrm{AU}) \approx 134 \mathrm{~km} \mathrm{~s}^{-1}$ could explain the blue shifted component of the broad line wings but the red component, i.e. the emitting material moving away from the observer, is difficult to explain for a disk that is oriented almost face-on. In this case, the wind moving away from the observer is covered by the disk. Accretion columns along which material moves from the disk onto the star or collimated jets leaving the star at the poles are possible but rather speculative scenarios for the cause of the broad observed line profile. 


\section{Chapter 4}

\section{$3 \mathrm{D}$ models}

Circumstellar disks are in all respects three dimensional objects. Not only material but also radiation is transported in the radial direction in contrast to the modelling approach described in the previous Chapters. In the $1+1 \mathrm{D}$ models the transport equation is only calculated in the vertical direction and the stellar irradiation is treated by setting the outer boundary condition of the 1D plane-parallel (vertical) radiative transfer equation. Hence, any radial radiative transfer is neglected in this approach. The irradiation simplification in the 1D case is a good approximation at radii $R>R_{\text {in }}$, i.e. where direct irradiation effects can be neglected. As shown in the previous Chapter good model fits can be achieved this way assuming axisymmetric disks and neglecting any form of radiation transport between the disk annuli.

However, any model disk setup that deviates from axial symmetry or that includes disk gaps or direct irradiation of the central star demands the treatment of the irradiation field in all three spatial directions. In the following Section the 3D radiative transfer module (3DRT from here on; see Hauschildt \& Baron, 2006) that is used in this work to describe the radiation field in disks is introduced. Furthermore, the influence of density waves and planet formation structures on the line profile, and hence the direct spectral detectability of forming planets in disks, is investigated.

\subsection{Radiative transfer}

In a three dimensional setup the Euler-frame radiative transfer equation (RTE) reads

$$
\vec{n} \cdot \nabla I_{\nu}(\vec{r}, \vec{n}, \nu)=-\chi_{\nu}(\vec{r}, \nu) I_{\nu}(\vec{r}, \vec{n}, \nu)+\eta_{\nu}(\vec{r}, \nu) \quad,
$$

where $I_{\nu}(\vec{r}, \vec{n}, \nu)$ is the intensity at a frequency $\nu$, at a position $\vec{r}$, and in the direction described by the unit vector $\vec{n}$. The derivative of the intensity in Cartesian coordinates reads

$$
\nabla=\left(\frac{\partial}{\partial x}, \frac{\partial}{\partial y}, \frac{\partial}{\partial z}\right)^{\mathrm{T}}
$$

The direction $\vec{n}$ can be described by two angles $(\theta, \varphi)$. The mean intensity $J$ can be calculated from the source function using the operator splitting technique described Sect. 2.7

The formal solution of the radiative transfer equation in 3DRT can be calculated using a shortcharacteristic (SC, Olson \& Kunasz, 1987) or a long-characteristic (LC) method (see Fig. 4.1 for a schematic plot of SCs and LCs in a Cartesian grid). The short-characteristic starts at the center of a volume element (voxel) and ends at the point closest to the center of the next voxel. A long-characteristic is followed through the grid continuously. The distance between start and 

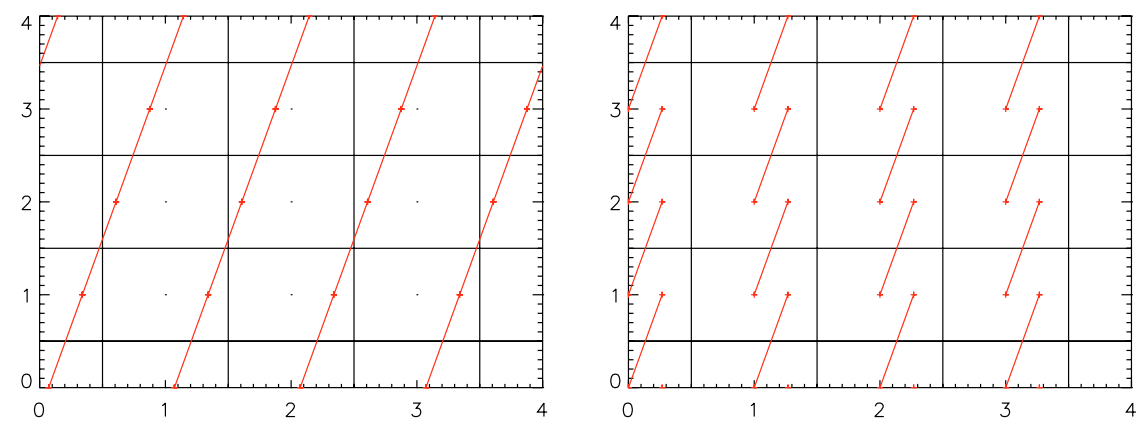

Figure 4.1: Schematic plots of different types of characteristics (taken from Hauschildt \& Baron, 2006). The left plot shows long-characteristics which are followed continuously through the grid while the right plot shows short-characteristics which always start at the center of a voxel. The plus signs mark points between which the distance $\Delta s$ for the optical depth calculations are measured.

end point of a characteristic between two grid points is calculated and determines the step in optical depth $\Delta \tau$. A piece-wise parabolic (PPM) or piece-wise linear (PLM) interpolation and integration of the source function can be used to compute the formal solution along a characteristic (Olson \& Kunasz, 1987). Auer (2003) elaborates on different interpolation techniques and points out that high-order non-local interpolations may lead to new local-extrema which cannot be physically justified. Hence, a piece-wise linear interpolation is used whenever the change in the source function is greater than some given value (typically $\Delta S>10^{3}$ ) or if the characteristic goes through very optically thin media $\left(\Delta \tau<10^{-3}\right)$.

Along a characteristic that is described by the angles $(\theta, \varphi)$ the RTE simply reads

$$
\frac{d I}{d \tau}=I-S
$$

The numerical solution of this equation applied here is described in the literature by Olson \& Kunasz (1987) and can be written as

$$
\begin{aligned}
& I\left(\tau_{i}\right)=I\left(\tau_{i-1}\right) \exp \left(\tau_{i-1}-\tau_{i}\right)+\int_{\tau_{i-1}}^{\tau_{i}} S(\tau) \exp \left(\tau-\tau_{i}\right) d \tau \\
& I\left(\tau_{i}\right)=I_{i-1} \exp \left(-\Delta \tau_{i-1}\right)+\Delta I_{i}
\end{aligned}
$$

where the index $i$ denotes a point along the characteristic and $\Delta \tau_{i}$ is obtained by piece-wise linear interpolation of the total extinction $\chi$, i.e.

$$
\Delta \tau_{i-1}=\frac{\chi_{i-1}+\chi_{i}}{2} \Delta s
$$

where $\Delta s>0$ is the geometrical step width. In this nomenclature for the optical depth $\tau_{i-1} \leq \tau_{i}$ and $\tau_{1}:=0$ is always true. Each characteristic is tracked all the way through the grid of voxels. A characteristic is started at the center of every voxel which is located on the boundary of the grid that faces the direction of the characteristic.

The integral in Eq. (4.4), which is expressed in discretised form $\Delta I$ in Eq. (4.5), is computed using linear or parabolic polynomials, i.e.

$$
\Delta I_{i}=\alpha_{i} S_{i-1}+\beta_{i} S_{i}+\gamma_{i} S_{i+1}
$$


with

$$
\begin{aligned}
\alpha_{i} & =e_{0 i}+\left[e_{2 i}-\left(\Delta \tau_{i}+2 \Delta \tau_{i-1}\right) e_{1 i} /\left[\Delta \tau_{i-1}\left(\Delta \tau_{i}+\Delta \tau_{i-1}\right)\right]\right. \\
\beta_{i} & =\left[\left(\Delta \tau_{i}+\Delta \tau_{i-1}\right) e_{1 i}-e_{2 i}\right] /\left[\left(\Delta \tau_{i-1} \Delta \tau_{i}\right]\right. \\
\gamma_{i} & =\left[e_{2 i}-\Delta \tau_{i-1} e_{1 i}\right] /\left[\Delta \tau_{i}\left(\Delta \tau_{i}+\Delta \tau_{i-1}\right)\right]
\end{aligned}
$$

for the parabolic interpolation and

$$
\begin{aligned}
\alpha_{i} & =e_{0 i}-e_{1 i} / \Delta \tau_{i-1} \\
\beta_{i} & =e_{1 i} / \Delta \tau_{i-1} \\
\gamma_{i} & =0
\end{aligned}
$$

for the linear interpolation, where

$$
\begin{aligned}
& e_{0 i}=1-\exp \left(-\Delta \tau_{i-1}\right) \\
& e_{1 i}=\Delta \tau_{i-1}-e_{0 i} \\
& e_{2 i}=\left(\Delta \tau_{i-1}\right)^{2}-2 e_{1 i} .
\end{aligned}
$$

In order to avoid large memory requirements, the mean intensity of the radiation field $J$ at a given point $\left(i_{x}, i_{y}, i_{z}\right)$ in the grid is calculated using a Monte Carlo type integration by replacing

$$
J\left(i_{x}, i_{y}, i_{z}\right)=\frac{1}{4 \pi} \int_{0}^{2 \pi} \int_{0}^{\pi} I\left(i_{x}, i_{y}, i_{z}\right) \sin \theta d \theta d \varphi
$$

with

$$
J^{\mathrm{MC}}\left(i_{x}, i_{y}, i_{z}\right)=\frac{1}{2 \pi^{2}} \sum_{j \in(\theta, \varphi)} I_{j}\left(i_{x}, i_{y}, i_{z}\right) \sin \theta_{j}
$$

where the sum extends over all solid angle points. The Monte Carlo integration is especially advantageous if RT in moving media is considered because the solid angle points can vary from grid point to grid point. In the case of static media, the Monte Carlo integration provides results close to those of the deterministic quadrature.

In order to calculate the mean intensity $J$ when scattering is present, the operator splitting method is applied and the approximate lambda operator $\Lambda^{\star}$ has to be determined (see Sect. [2.7). Olson \& Kunasz (1987) showed how to construct a non-local tri-diagonal $\Lambda^{\star}$ operator from the coefficients $\alpha, \beta$, and $\gamma$ for the 1D RT problem. The non-local operators show good convergence properties over a local $\Lambda^{\star}$ and also avoid the possibility of false convergence. While in the 1D case the tri-diagonal $\Lambda^{\star}$ operator at each point in the atmosphere only needs to account for interactions with the two direct neighbours, in the 3D case the nearest neighbour $\Lambda^{\star}$ has to include the interaction with 26 surrounding voxels. Hence, for each grid point $(26+1) \Lambda^{\star}$ entries have to be stored. Hauschildt et al. (1994) describe how to determine the $\Lambda^{\star}$ operator of arbitrary bandwidth. The construction of the $\Lambda^{\star}$ is described in detail by Olson \& Kunasz (1987). The elements of a row of the $\Lambda^{\star}$ operator for a voxel with the coordinates $\left(i_{x}, i_{y}, i_{z}\right)$ can be calculated by performing a formal solution and setting the intensity at the boundary $I_{1}=0$ and $S\left(i_{x}, i_{y}, i_{z}\right)=1$. 
For a single characteristic going through a $3 \mathrm{D}$ grid, the contributions to the $\Lambda^{\star}$ at a voxel $j$ are obtained by

$$
\begin{aligned}
\Lambda_{i, j} & =0 & & \text { for } i<j-1 \\
\Lambda_{j-1, j} & =\gamma_{j-1} & & \text { for } i=j-1 \\
\Lambda_{j, j} & =\Lambda_{j-1, j} \exp \left(-\Delta \tau_{j-1}\right)+\beta_{j} & & \text { for } i=j \\
\Lambda_{j+1, j} & =\Lambda_{j, j} \exp \left(-\Delta \tau_{j}\right)+\alpha_{j+1} & & \text { for } i=j+1 \\
\Lambda_{i, j} & =\Lambda_{i-1, j} \exp \left(-\Delta \tau_{i-1}\right) & & \text { for } \quad i>j+1
\end{aligned}
$$

where $i$ is the index of the voxels along the characteristic. The contributions to $\Lambda^{\star}$ at a voxel $\left(i_{x}, i_{y}, i_{z}\right)$ for each $(\theta, \varphi)$ characteristic are integrated in the same way as the mean intensity $J$, either deterministic or by a Monte Carlo method. For a nearest neighbour $\Lambda^{\star}$ the calculation of $\Lambda_{i, j}$ (see Eq. (4.13e) ) is terminated at $i=j+1$.

\subsection{Grid setup}

The 3DRT framework allows to calculate the radiative transfer in three different coordinate systems. Depending on the physical problem, the appropriate coordinates can be chosen, i.e. Cartesian, spherical, or cylindrical. The number of grid points is given as input.

In the case of a Cartesian coordinate system $\mathrm{nx}$, ny, and $\mathrm{nz}$ are set generating a total of $(2 * \mathrm{nx}+1) *(2 * \mathrm{ny}+1) *(2 * \mathrm{nz}+1)$ voxels and the grid points are spaced equidistantly. In the case of disks, the geometrical step width, i.e. the shortest distance between two grid points, is set in $\mathrm{x}$ - and $\mathrm{y}$-direction by the outer radius of the disk region to be modelled, hence $\Delta \mathrm{x}=\Delta \mathrm{y}=$ $2 f R_{\text {out }} /(2 * \mathrm{n}+1)$, with $\mathrm{n}=\mathrm{nx}=\mathrm{ny}$. In the $\mathrm{z}$-direction the geometrical step width is determined by $\Delta \mathrm{z}=2 f H_{\max } /(2 * \mathrm{nz}+1)$, where $H_{\max }$ is the maximum height of the disk. The factor $f$ is to avoid that the edge of the disk hits the border of the grid and is set to $f=1.1$. The Cartesian grid is not well suited for disk geometries in the equidistant form because many of the grid points are empty, e.g. between the star and the disk, so that memory and computational time is not used efficiently.

The cylindrical coordinate system is better suited for the geometry of disks. The number of voxels is set by the input parameters describing the radial, azimuthal, and vertical grid: nr, nt, and $\mathrm{nz}$ making a total of $(2 * \mathrm{nr}+1) *(2 * \mathrm{nt}+1) *(2 * \mathrm{nz}+1)$ points. Here the coordinates of the radial and vertical grid points can be chosen manually and non-equidistant. Due to the flaring in disks (the increase of height with radius) the step width in vertical direction is chosen to be constant. The border between two voxels is set halfway between two points, i.e. $R_{i}^{\mathrm{b}}=\left(R_{i}^{\mathrm{v}}+R_{i+1}^{\mathrm{v}}\right) / 2$, with $i \in\{-\mathrm{nr},-\mathrm{nr}+1, \ldots,+\mathrm{nr}\}$. Therefore, the innermost voxel with $R_{-\mathrm{nr}}^{\mathrm{v}}=0$ has a border at $R^{\mathrm{b}}=R_{\star}$ and "contains" the star. The radial points which are meant to hold the physical structure of the disk are placed between $R_{\text {in }}$ and $R_{\text {out }}$ equidistantly on a logarithmic grid, viz.

$$
R_{i}=R_{\mathrm{in}} \cdot 10^{\left((\mathrm{nr}-3+i)^{\eta} \Delta \sigma\right)} \quad, \quad i \in\{-\mathrm{nr}+3,-\mathrm{nr}+2, \ldots, \mathrm{nr}\}
$$

with

$$
\Delta \sigma=\frac{\log \left(R_{\text {out }}\right)-\log \left(R_{\text {in }}\right)}{(2 \cdot \mathrm{nr}-3)^{\eta}}
$$




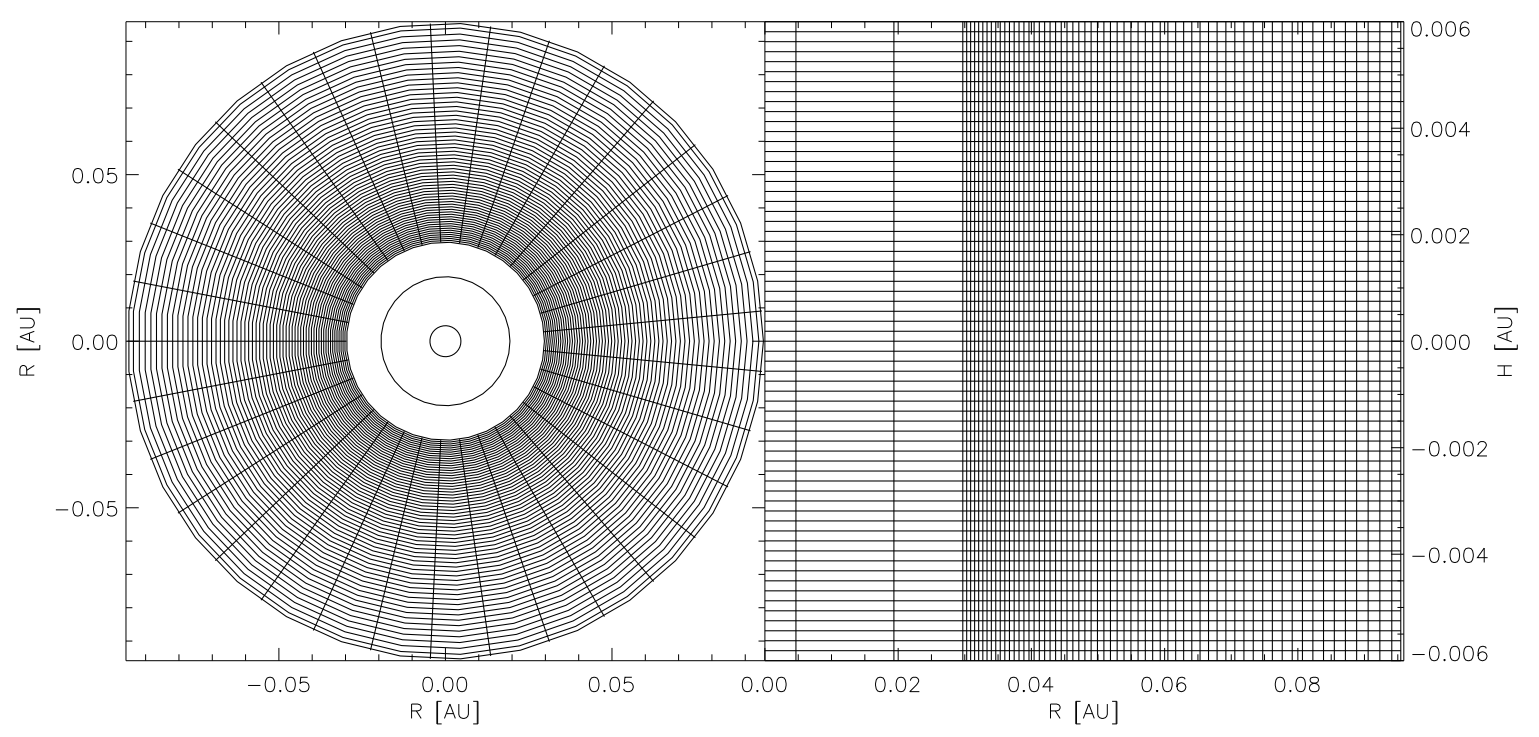

Figure 4.2: Face-on (left) and vertical cut through the grid (right) view of the cylindrical voxel grid with $\mathrm{nr}=\mathrm{nz}=32$ and $\mathrm{nt}=16$.

where $\eta$ controls the increase in step width $\Delta r$ with radial index $i$ and is by default set to $\eta=1$. A larger value of $\eta$ makes a finer $r$ spacing close to the star and a coarser grid at larger radii. The point $R_{-\mathrm{nr}+2}$ and $R_{\mathrm{nr}}$ are set to guarantee that for the boundaries $R_{-\mathrm{nr}+2}^{\mathrm{b}}=R_{\mathrm{in}}$ and $R_{\mathrm{nr}}^{\mathrm{b}}=R_{\text {out }}$. A sketch of a cylindrical grid for circumstellar disks is shown in Fig. 4.2.

Once the grid is setup, the physical structure has to be determined for each voxel. For 3D disks, a set of $1 \mathrm{D}$ vertical structure models is interpolated using a piece-wise parabolic method (see Fig. 4.3 for interpolation quality). In a first step of the interpolation procedure, a given variable, known for a set of $n_{\text {rings }} 1 \mathrm{D}$ disk structures of different radii $R$, is interpolated for a number of $n_{R}=\left(n_{\text {inter }} \times n_{\text {rings }}\right)$ grid points spaced equidistantly between $R_{\text {in }}$ and $R_{\text {out }}$ for each layer. In a next step, for each of the $n_{R}$ grid points the structure in vertical direction, i.e. between the layers, is determined also for $n_{z}=n_{R}$ points equidistantly between $z=0$ and $z=z_{\max }$. In a final step, the $\left(n_{R} \times n_{z}\right)$ grid is used to fill the voxel grid by employing linear interpolation between the finely spaced points. Generally it is assumed that the disk is axisymmetrical and mirror-symmetrical with respect to the midplane.

The quantities that are needed to solve the 3D RT problem are the temperature $T$ which sets the Planck intensity $B$ at every grid point, the opacity $\chi$, the source function $S$, and the thermalisation parameter $\epsilon$. In the case of grey RT the opacity is simply set to the mean Rosseland opacity $\chi_{\text {ross }}$ and the initial source function $S=B$. Furthermore, the voxels filled by the central star have to be determined. In the cylindrical geometry case, the star fills the innermost voxel in radial direction and the number of grid points in vertical direction $n_{z}^{\star}$ are determined according to $n_{z}^{\star} \Delta z \leq R_{\star}$. The star is assumed to radiate like a blackbody of stellar effective temperature $T_{\text {eff }}^{\star}$ and every characteristic hitting a voxel "filled" by the star is automatically assigned the intensity $I_{\lambda}=B_{\star}(\lambda)$. In the grey case $B_{\star}=B\left[T_{\text {eff }}^{\star}, \lambda=1.2 \mu \mathrm{m}\right]$. 


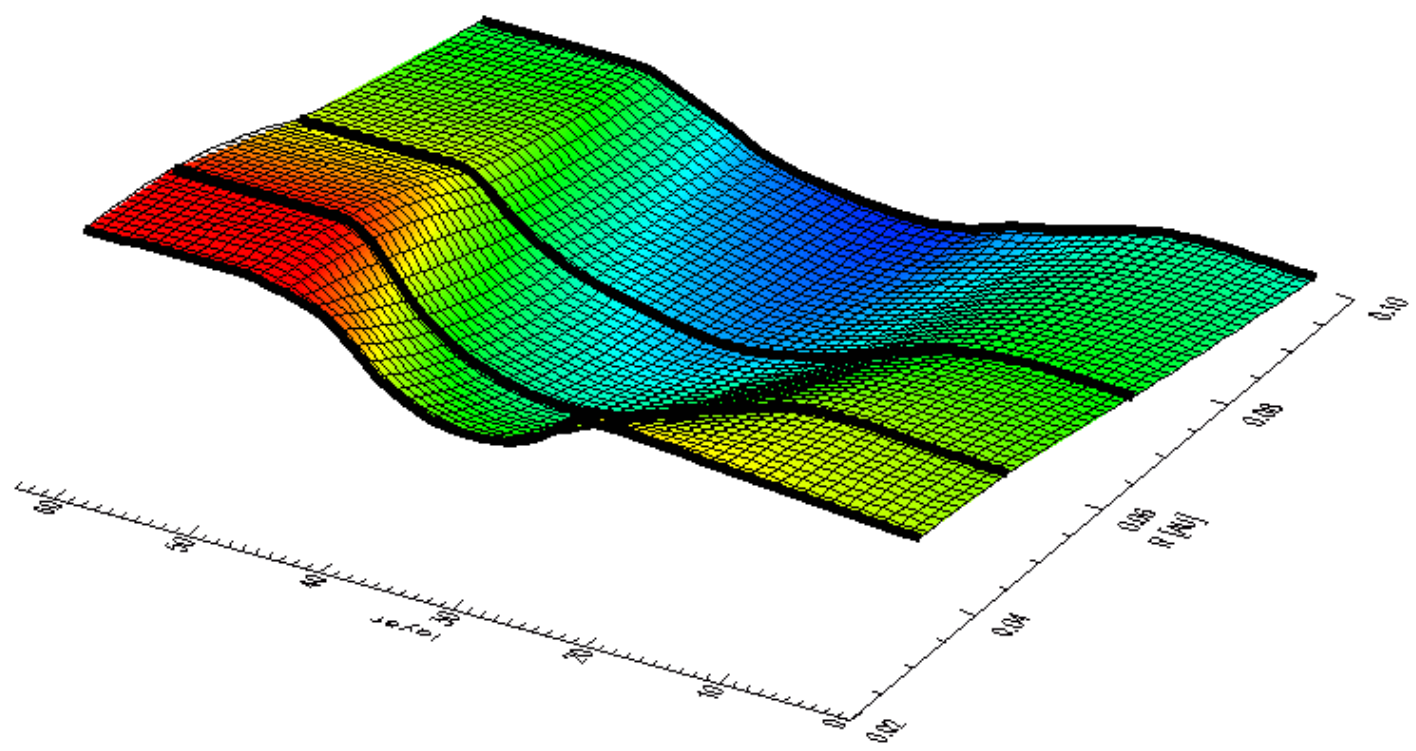

Figure 4.3: Surface plot of disk temperatures interpolated on a Cartesian grid (x-axis: radius $R, y$-axis: $1 \mathrm{D}$ layer index). The coloured surface is a plot of the temperature from the four given ring structures (colours and height correspond to temperature values). The mesh shows the temperature grid calculated from the four input structures with the same quadratic interpolation method as in 3DRT, the thick black lines are the original 1D structures.

\subsection{Line transfer in moving media}

The structures of the 1+1D models described in Chapter 2 are computed assuming disk matter to be at rest. Only for the calculation of the spectrum of the total disk seen under an inclination $i$ the rotational effects are considered. For a given inclination $i$ the spectrum is rotationally broadened assuming a geometrically thin disk, i.e. the radiation originates in the uppermost layers, and applying Doppler shifts for a number of annulus sections divided in azimuth $\varphi$ with velocities $u(i, \phi)=u_{\mathrm{Kep}} \sin (i) \cos (\varphi)$. This approach is only an approximation because the line origin is not in the top layer but deeper in the atmosphere (see Sect. 2.12). Therefore, the photons can be trapped in optically thick lines and escape more easily in regions where large Doppler gradients along the photon path are present (Horne \& Marsh, 1986). To investigate these effects, full 3D line transfer simulations in rotating disks are necessary. Early models of Adam (1990) were calculated using the two-level-atom approach and complete re-distribution in a disk with homogeneous temperature and density structure. The RTE was solved on an equidistant Cartesian grid using a first order finite volume technique including scattering.

While Adam (1990) modelled homogeneous disks for CV systems or AGNs, the approach presented in this Section concentrates on spatially dependent protoplanetary disk structures. The RTE is solved using LCs in a cylindrical grid more adequate for the use with disks which 
leads to a higher spatial resolution needed to resolve the line shifts between neighbouring voxels. The disk has a grey continuum opacity $\chi_{c}=\chi_{\text {ross }}$ which can be parametrised to coherent and isotropic continuum scattering by

$$
\chi_{c}=\epsilon_{c} \kappa_{c}+\left(1-\epsilon_{c}\right) \sigma_{c},
$$

with $\epsilon_{c}$ being the continuum thermalisation parameter and $\kappa_{c}$ and $\sigma_{c}$ the continuum absorption and scattering coefficients. For the calculations presented here $\epsilon_{c}=1$. The line of the twolevel-atom is parametrised by the ratio of the profile averaged line opacity $\chi_{l}$ and the continuum opacity $\chi_{c}$ as well as the line thermalisation parameter $\epsilon_{l}$ (Baron \& Hauschildt, 2007). The line wavelength, the number of wavelength points for the line as well as the spacing of the wavelength in the line and in the surrounding continuum region can be set on input. The line profile function is assumed to be a Gaussian with the width $\sigma$ and is computed for each solid angle point $(\theta, \varphi)$ and wavelength $\lambda$ for each voxel by

$$
\phi(\lambda)=\frac{p_{\text {norm }}}{\sqrt{\pi} \sigma} \exp \left(-\left[\frac{\tilde{\lambda}-\lambda_{0}}{\sigma}\right]^{2}\right),
$$

where $p_{\text {norm }}$ is a normalisation factor and $\tilde{\lambda}$ is the wavelength at which the photon of wavelength $\lambda$ sees the line profile function due to the Doppler shift. The shifted wavelength is determined by

$$
\tilde{\lambda}=\lambda\left(1+\frac{\vec{v} \cdot \vec{n}}{c}\right)
$$

where the velocity is given by

$$
\vec{v}(R, \varphi)=\left(\begin{array}{c}
v_{x}(R, \varphi) \\
v_{y}(R, \varphi) \\
v_{z}(R, \varphi)
\end{array}\right)=\sqrt{\frac{G M_{\star}}{R}}\left(\begin{array}{c}
-\sin (\varphi) \\
+\cos (\varphi) \\
0
\end{array}\right) .
$$

and taken in the direction of the characteristic $\vec{n}$. The line profile function $\phi_{\lambda}$ enters the total opacity

$$
\chi_{\lambda}=\chi_{\lambda}^{c}+\chi_{\lambda}^{l}=\chi_{\lambda}^{c}+g_{\lambda}^{l} \chi_{\lambda}^{c} \phi_{\lambda}
$$

where $g_{l}=\chi_{l} / \chi_{c}$ is the parametrised line strength and the line source function $S_{l}$ is given by

$$
S_{l}=\left(1-\epsilon_{l}\right) \bar{J}+\epsilon_{l} B
$$

where $\bar{J}=\int \phi_{\lambda} J_{\lambda} d \lambda$ is the profile integrated mean intensity. The total wavelength-dependent source function is then given by

$$
S_{\lambda}=\frac{\kappa^{c} B_{\lambda}+\sigma^{c} J_{\lambda}+\chi_{\lambda}^{l}\left((1-\epsilon) \bar{J}+\epsilon B_{\lambda}\right)}{\chi_{\lambda}}
$$

Since maximal velocities are of the order $v_{\max } \sim 100 \mathrm{~km} \mathrm{~s}^{-1}$, and hence $v / c \approx 3 \cdot 10^{-4} \ll 1$, relativistic effects can be neglected and the radiation field is calculated in the observer's frame. For each wavelength, the RTE is solved and the source function for the line scattering problem is determined. In the case of static media, the opacity and the source function are determined for each wavelength point once and the characteristics can be tracked through the grid for the 


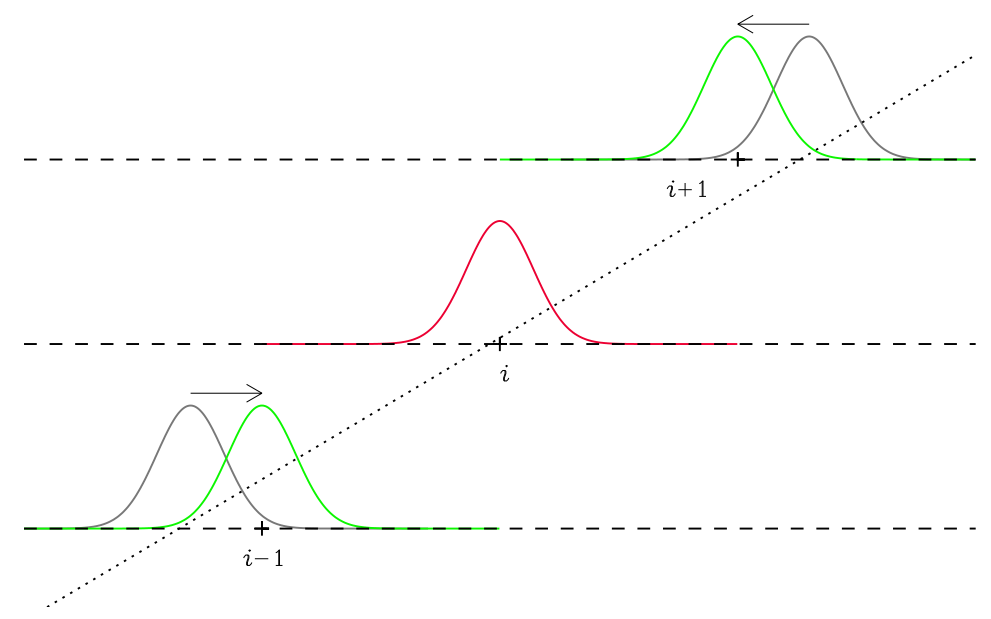

Figure 4.4: Visualisation of grid resolution effects. The coloured Gauss profiles represent the velocity-shifted line profiles and the dotted line is the characteristic. The grey profiles are the line profiles in a steady disk. If the grid resolution is too coarse, i.e. when the red profile is missing, the intensity at voxel $i+1$ is not influenced by the line if velocity effects are considered.

set of angles $(\theta, \varphi)$ resulting in the solid angle space integrated values $J_{\lambda}$ and $\Lambda_{\lambda}^{\star}$. If matter, like in the case of rotating circumstellar disks, is moving, the opacity and source function at a given wavelength point are different at each point of the grid for different solid angle points $(\theta, \varphi)$ because of projection effects (see Eqs. (4.17) and (4.18)). Therefore, the two-level line transfer approach presented in Baron \& Hauschildt (2007) had to be rewritten to handle this problem, which is realised in the Eulerian formulation. The line profile integrated mean intensity $\bar{J}$ and the approximate lambda operator

$$
\bar{\Lambda}^{\star}=\int \phi_{\lambda} \Lambda_{\lambda}^{\star} d \lambda
$$

are then used to update the $\bar{J}$ via the standard iteration method (Eq. (2.45)) until convergence of the form

$$
\frac{2}{N} \sum_{i}\left|\frac{\bar{J}^{i}-\bar{J}_{\text {old }}^{i}}{\bar{J}^{i}+\bar{J}_{\text {old }}^{i}}\right|<\epsilon_{\text {acc }}
$$

is achieved. The accuracy in $\bar{J}$ is typically $\epsilon_{\text {acc }}=10^{-8}$.

An MPI parallelisation over wavelength is implemented which is highly efficient (Baron \& Hauschildt, 2007) and the code can be split up onto $N_{\mathrm{wl}}$ (number of wavelength points) CPUs. A parallelisation of solid angle space is not implemented for the disk version of the 3D line transfer code.

After the correct source function is determined, the line profile can be calculated for a given viewing angle $\alpha(\theta, \varphi)$ by performing a formal solution for the solid angle point $(\theta, \varphi)$. For an axisymmetric disk configuration the azimuthal angle $\varphi$ is degenerate.

\subsubsection{Grid resolution}

Due to the rotation of matter around the central star with Keplerian velocity, the line profile function is shifted in wavelength between two neighbouring voxels along the path of a characteristic 


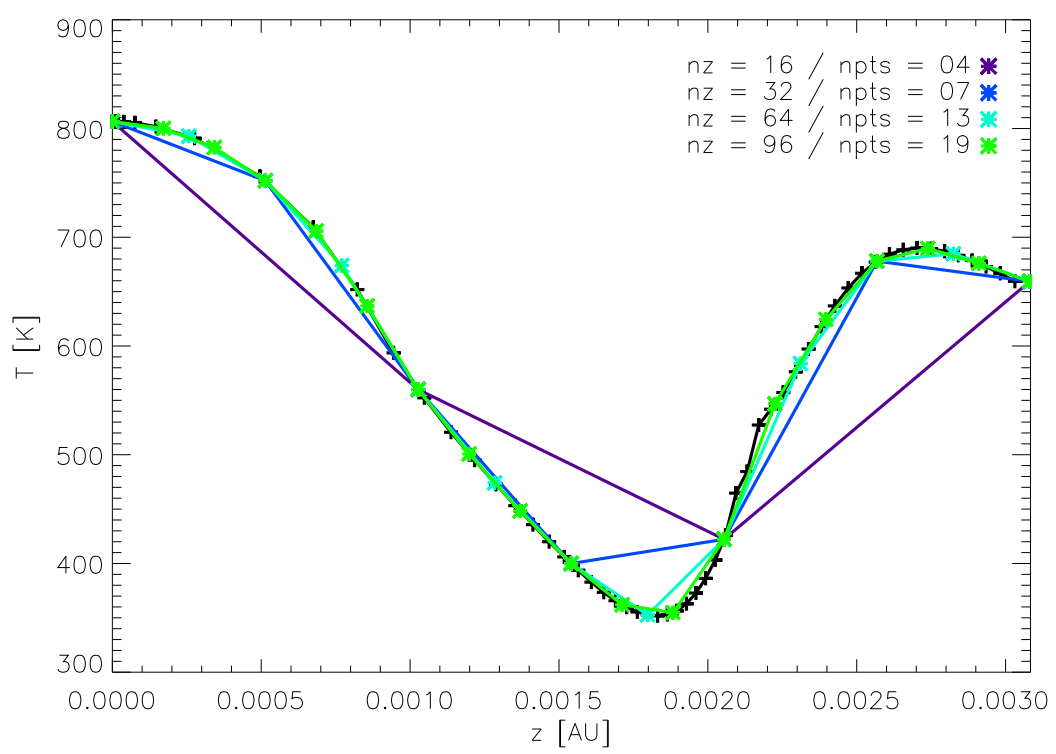

Figure 4.5: Comparison between an input 1D temperature structure model at $R=$ $R_{\text {in }}$ with 64 points vertical grid points (black line) and interpolated $3 \mathrm{D}$ structures with different number of grid points in vertical direction (coloured lines). The variable nz in the legend gives the number of vertical grid points and npts is the number of points that actually hold the disk structure. At $R=R_{\text {in }}$ the ratio of npts and $\mathrm{nz}$ is the smallest.

(see Fig. 4.4). In order to make sure that the line is well sampled, the grid steps have to be adequately small. A typical $\mathrm{CO}$ line at $\lambda=4.5 \mu \mathrm{m}$ has a full width at half maximum (FWHM) of $\sigma=1 \AA$ and for a sampling of two points per line the maximum step in velocity should not exceed

$$
\Delta v_{\max }=\frac{\sigma}{2 \lambda} c \approx 3.5 \mathrm{~km} \mathrm{~s}^{-1}
$$

where $c$ is the speed of light. An upper boundary for the maximal Doppler shift can be derived assuming that the maximum change in velocity occurs when the characteristic proceeds from a voxel at the inner disk radius $R_{\mathrm{in}}$ and $\varphi_{0}$ to the next voxel at $R_{\mathrm{in}}+\Delta R$ and $\varphi_{0}+\Delta \varphi$. Then the change in $v$ is always smaller than

$$
\Delta v \leq v_{\text {in }}(1-\cos \Delta \varphi)+\Delta v_{r},
$$

with $\Delta v_{r}$ being the maximum change of the Keplerian velocity in radial direction. For a typical disk with $M_{\star}=0.5 M_{\odot}$ and $R_{\text {in }}=0.05$ AU the inner velocity is $v_{\text {in }}=94 \mathrm{~km} \mathrm{~s}^{-1}$. In this case, a minimum of $\mathrm{nt}=32$ or 65 azimuthal grid points and $\mathrm{nr}=16$ or 33 radial grid points is sufficient to resolve the line along a characteristic.

Furthermore, the resolution in solid angle space has to be sufficient to resolve the line, i.e. in a given voxel the change in projected velocity between two neighbouring solid angle points may not exceed $\Delta v_{\max }$. Hence, at least 24 solid angle points are needed in $\theta$ and $\varphi$ to resolve the line in the example disk setup given above.

Obviously, the resolution in $z$-direction (vertical disk structure) should not be chosen too poor either. This would not have a significant influence on the velocity coverage ( $v$ is constant in $z$ ) 

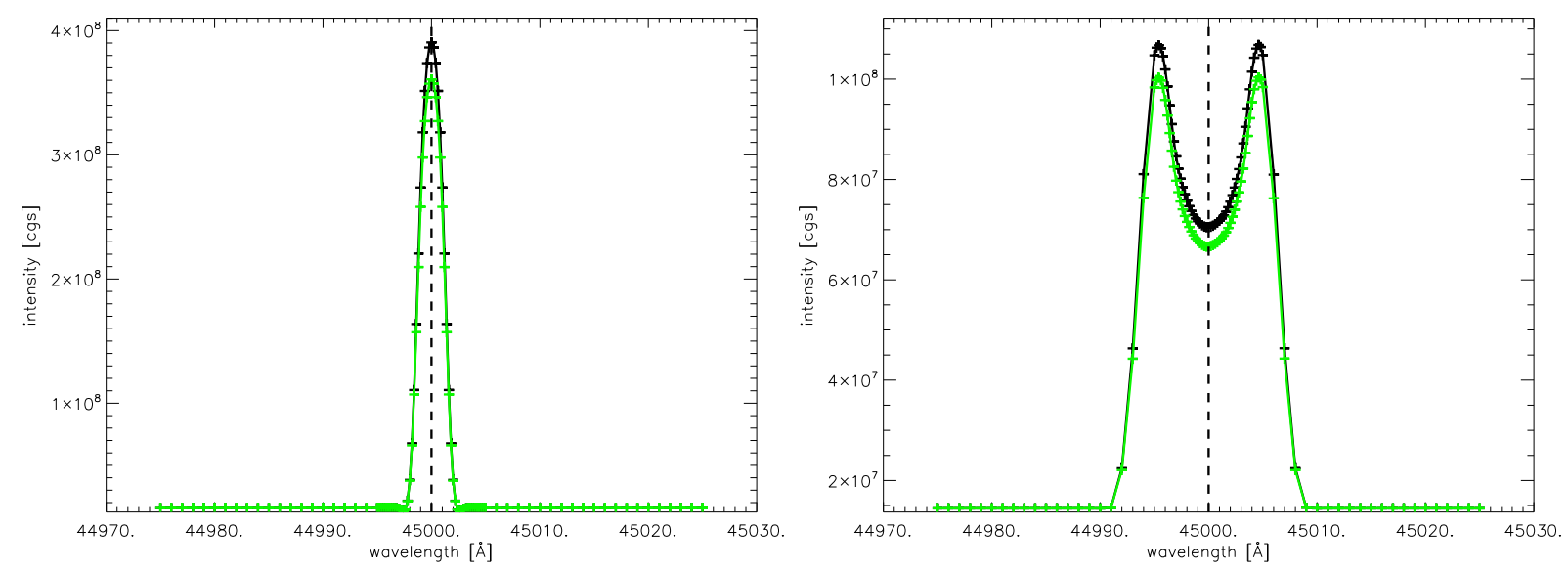

Figure 4.6: $3 \mathrm{D}$ line profiles for disk structures with line thermalisation parameter $\epsilon_{l}=$ 1.0 (black line) and $\epsilon_{l}=0.8$ (green line). The left plot is for a disk seen under an inclination of $i=0^{\circ}$ and the right one for $i=30^{\circ}$. Only radiation leaving the surface of the disk but not from the inner disk wall is considered.

but the structure needs to be resolved to get realistic results (see Fig. 4.5). Therefore, at least 10 of the 64 layer points used in the 1D model should be chosen, which are spaced equidistantly in $\log m$ and not in $z$.

\subsubsection{Axisymmetric disks}

In a first step, the disk is assumed to be axisymmetric and the line profile is calculated for different viewing angles. The "test disk" is interpolated from eight $1 \mathrm{D}$ models with the following parameters of a typical CTTS inner disk: $R=0.050,0.061,0.074,0.091,0.110,0.135,0.164$, and $0.200 \mathrm{AU}$, $R_{\star}=1 R_{\odot}, M_{\star}=0.5 M_{\odot}, T_{\text {eff }}=4060 \mathrm{~K}, \dot{M}=10^{-9} M_{\odot} \mathrm{yr}^{-1}$, and $R e=5 \cdot 10^{5}$. The line strength is set to $g_{l}=10^{3}$.

In Fig. 4.6 a comparison between models with a line scattering parameter of $\epsilon_{l}=1.0$ and $\epsilon_{l}=0.8$, which is an average value for a CO $v=1-0$ line in the wavelength region around $\lambda=4.5 \mu \mathrm{m}$, are shown for inclinations of $i=0^{\circ}$ and $i=30^{\circ}$. The disk has a resolution of $\mathrm{nr}=\mathrm{nt}=32, \mathrm{nz}=64$ and $32^{2}$ solid angle points. As shown in the previous Section, this disk setup is sufficient to resolve the spectral line when considering Keplerian rotation of the disk. For the line profiles shown only radiation leaving the top of the disk atmosphere is considered; emission from the inner disk wall is neglected in order to allow the comparison with 1+1D line profiles calculated as described in Sect. 2.10 (see Fig. 4.9] for illustration). The difference between 3D line profiles with and without taking into account the inner disk wall as emitting source is shown in Fig. 4.7. The difference in intensity is basically caused by a raise of the continuum that can be attributed to the inner disk wall seen when looking at the disk at an inclination $i>0^{\circ}$. The pure line flux is basically unaffected by the different setups. The plots in Fig. 4.6 show that a disk with $\epsilon_{l}=0.8$ loses some of the line emission as a result of line scattering as expected from the results of Baron \& Hauschildt (2007). Since $\epsilon_{l}$ is constant in the disk and most of the line emission stems from the inner regions of the disk (see Fig. 4.8), the loss in line intensity for $\epsilon_{l}=0.8$ is approximately the same over the whole line width. Therefore, the following results are all limited to the case where $\epsilon_{l}=1$ which saves about a factor of ten in computing time and does not change the results of the calculations qualitatively. 


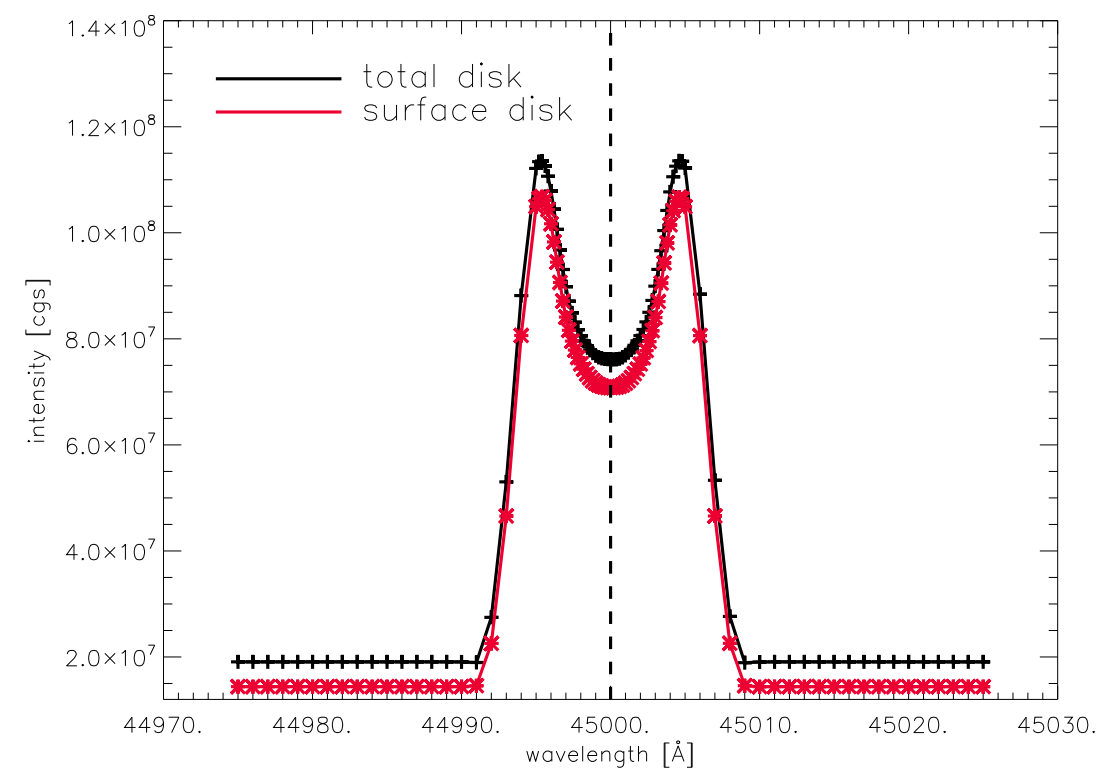

Figure 4.7: Difference between 3D spectral lines emitted just from the disk surface (red line) and the total disk including the inner disk wall (black line) for an inclination of $i=30^{\circ}$.

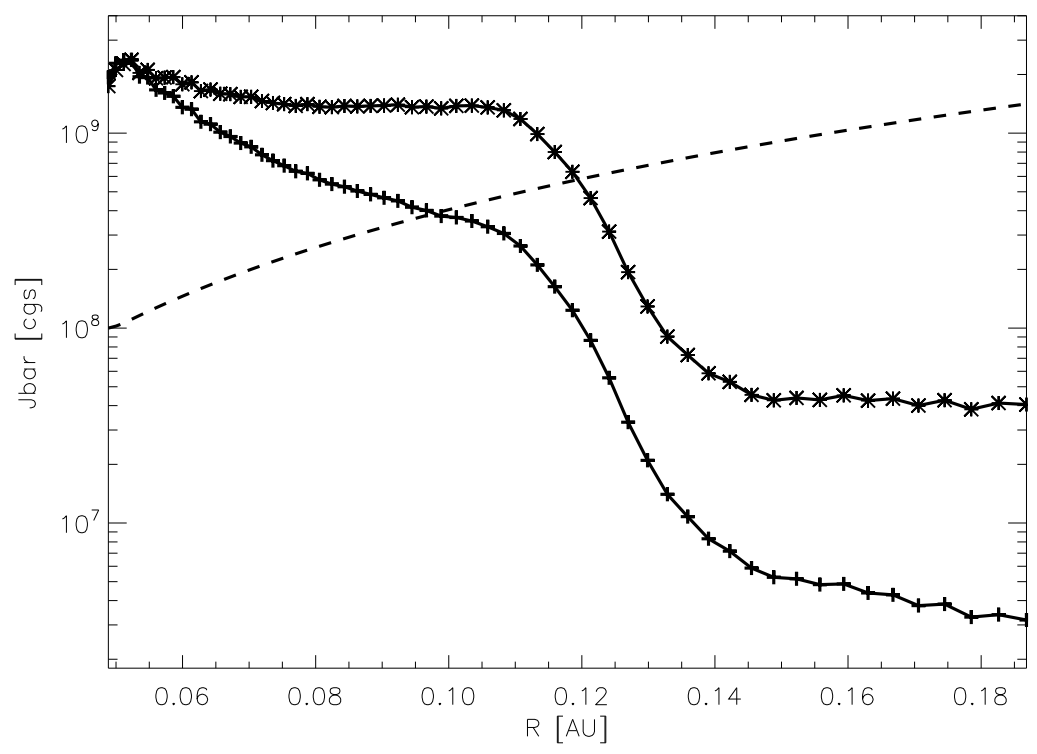

Figure 4.8: Run of the line-integrated mean intensity $\bar{J}$ at the top of the disk atmosphere with radius $R$ for single grid points (crosses) and annulus-integrated values (asterisk; scaled to the maximum grid intensity). The dashed line shows the area of each annulus in arbitrary units. Most of the line emission stems from the very inner region of the simulated disk with a steep drop off at $R \approx 0.12 \mathrm{AU}$. 


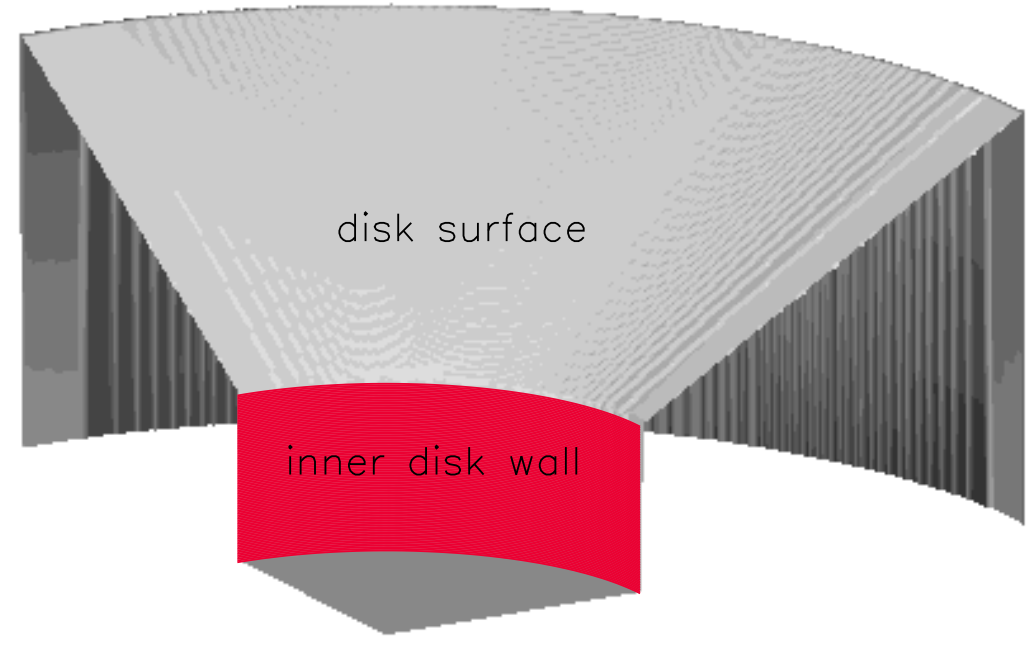

Figure 4.9: Illustration of disk emission regions considered for line profile calculations. Shown is a quarter of a disk from the midplane up to the disk surface. The grey region labelled "disk surface" is the emission source taken into account in the $1+1 \mathrm{D}$ models. The red "inner disk wall" contributes additional emission in the case of the more realistic full 3D RT.

\section{Comparison between 1D and 3D line profiles}

In the calculations of the $1 \mathrm{D}$ disk radiation field described in Chapter 2, the rotation of matter around the center of mass is neglected. The effect of line broadening due to moving media is taken into account in a later step where it is assumed that the disk is flat and that the radiation observed originates from the surface of the disk (see Sect. 2.10). In order to evaluate whether or not these simplifications are applicable, 3D lines will be compared with lines broadened according to the $1 \mathrm{D}$ method.

Horne \& Marsh (1986) noticed in their work that line photons which are trapped in an optically thick emission layer can escape more easily in directions along which the Keplerian flow produces large Doppler gradients. If this is the case for protoplanetary disks with inclinations of $i<40^{\circ}$, which are typical inclinations for the disks observed, this effect should be detectable by comparing 3D lines taking into account velocity broadening and 1D lines broadened subsequently. Horne \& Marsh (1986) as well as Adam (1990) calculated line profiles for compact CV disks and the former found differences in the profiles between optically thin and optically thick emission lines only at large inclinations $i \gtrsim 60^{\circ}$.

In order to perform the comparison between $1 \mathrm{D}$ and $3 \mathrm{D}$ line profiles, 3D line profiles for NR annuli with the same width in radius $\Delta R=\left(R_{\text {out }}-R_{\text {in }}\right) / \mathrm{NR}$ are computed for $i=0^{\circ}$. In this face-on view case, the velocity broadening of the lines is not present because projected velocities are zero. Subsequently, 3D line profiles for inclinations $i>0^{\circ}$ are computed taking into account only the radiation that leaves the top of the disk atmosphere but not from the inner disk wall to allow the comparison with the 1D models. In a next step, the line profiles for the NR rings are broadened according to Eq. (2.66) assuming a velocity of $u_{i}=u_{\mathrm{Kep}}\left(R_{i}\right)$ with $R_{i}=R_{\text {in }}+(i-1 / 2) \Delta R, i \in\{1,2, \ldots, \mathrm{NR}\}$. Since the intensity varies when changing the inclination, the total disk-integrated and continuum-subtracted line flux is scaled to the value 


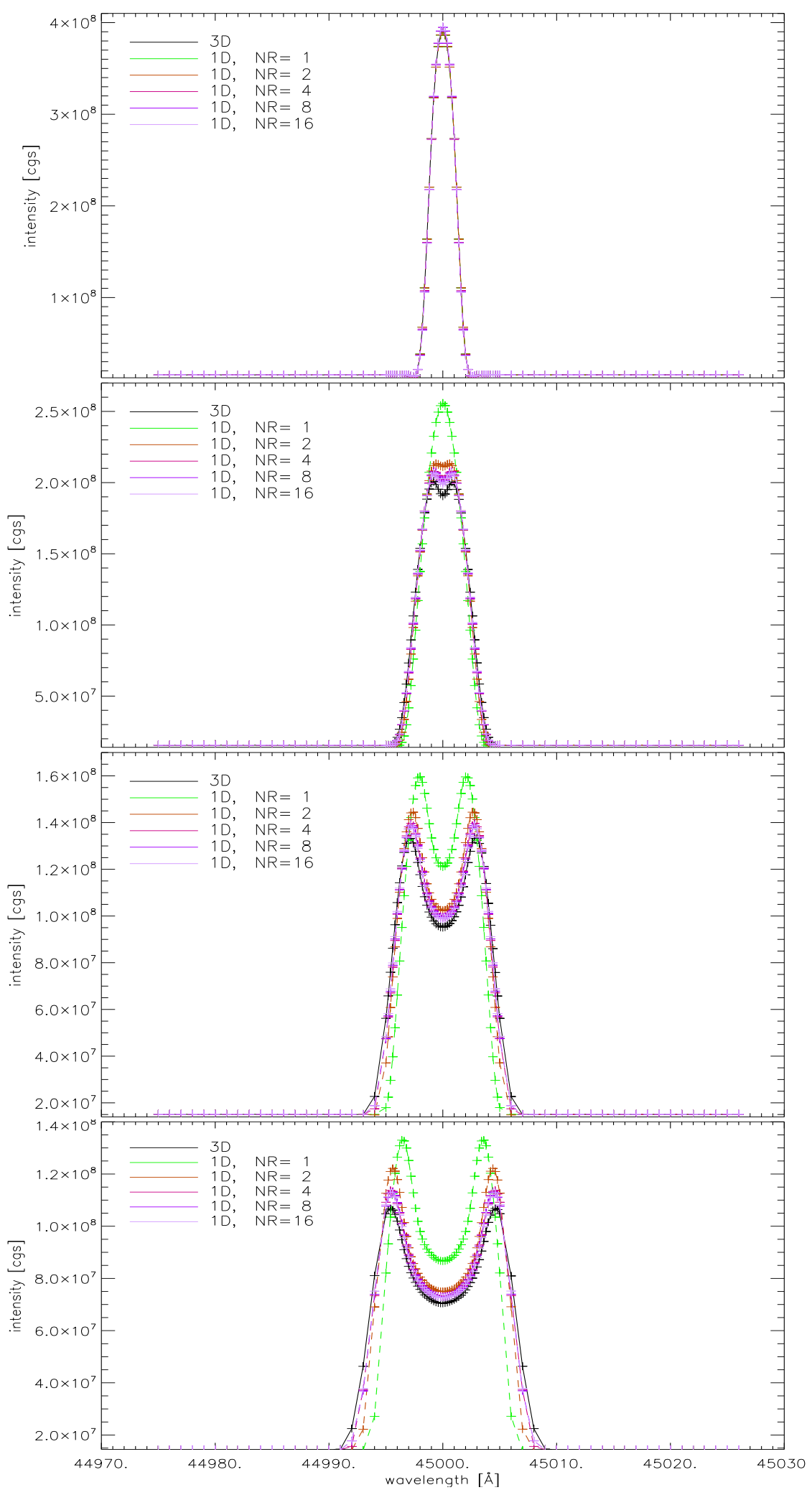

Figure 4.10: Comparison between 1D and 3D line profiles for inclinations of $i=0^{\circ}$, $10^{\circ}, 20^{\circ}$, and $30^{\circ}$ (top to bottom). The black line is the $3 \mathrm{D}$ spectral line calculated from the 3D structure where only intensities from the top of the atmosphere (no inner disk wall contributions) are taken into account. The coloured lines are the $i=0^{\circ} 3 \mathrm{D}$ line broadened according to Eq. (2.66) assuming NR annuli. 
calculated from the 3D line profile and the continuum flux is replaced by that of the 3D line in order to allow a comparison. In Fig. 4.10 3D line profiles for inclinations of $i=0^{\circ}, 10^{\circ}, 20^{\circ}$, and $30^{\circ}$ are compared to $1 \mathrm{D}$ line profiles using $\mathrm{NR}=1,2,4,8$, and 16 annuli. For an inclination of $i=0^{\circ}$ the line profiles are very similar as expected because no velocity broadening is present. For inclinations $i>0^{\circ}$ the general trend that 1D line profiles are narrower and have a higher maximal intensity can be observed. This effect is the strongest for the line with NR $=1$ because the Kepler velocity is a constant and average value for the whole disk and hence the line profile is broadened equally strong over the whole disk. Increasing the number of annuli to only two rings already improves the line shape significantly. For $\mathrm{NR}=4, \mathrm{NR}=8$ and $\mathrm{NR}=16$ the differences to the 3D model get even smaller and the $1 \mathrm{D}$ line shape seems to converge towards a final value. The residual difference seems to be the result of the physical full 3D RT treatment and the approximate 1D line broadening rather than a resolution problem in the $1 \mathrm{D}$ case. The latter problem can be excluded because from $\mathrm{NR}=4$ on a further disk ring refinement does not provide a significant improvement in the line shape compared to the $3 \mathrm{D}$ line.

From this comparison one can learn two things. First, a set of four 1D disk structures, in this case spanning a range in radius of $R=0.05-0.20 \mathrm{AU}$, is sufficient to accurately model the line shape in the $1+1 \mathrm{D}$ approximation. This is consistent with $1+1 \mathrm{D}$ test calculations for GQ Lup (see Sect. 3.1.2). Second, the 3D line is broader and less intense compared to a line with the same integrated flux but broadened subsequently.

\subsubsection{Non-axisymmetric disks}

The picture of a smooth and axisymmetric disk assumed in all previous calculations shown in this work is certainly an idealised one. In reality circumstellar disk will be non-axisymmetric due to e.g. clumping of material or planet formation. How such processes alter the appearance of a 3D spectral line shall be investigated in this Section by comparing it to the smooth and axisymmetric disk results.

\section{Density waves}

In this first step of modelling non-axisymmetric disks, a single wave running through the disk is considered. This wave can be interpreted as the density spiral running inward from a planet forming in the disk at a larger radius than the outer one considered for these line transfer calculations. Since this inner region creates the major flux output in the wavelength region of the dominant $\mathrm{CO}$ emission lines around $4.7 \mu \mathrm{m}$, neglecting the actual planet seems appropriate. In this model the planet would only be indirectly detectable.

In this simplified model it is assumed that the surface density of the wave is a certain factor $\delta=\Sigma_{\text {wave }} / \Sigma_{0}$ higher than that of the ambient region. An increase in surface density is supposed to be partitioned equally in the vertical direction of the disk. The opacity needed for the 3DRT simulations is scaled linearly with the surface density

$$
\kappa_{\text {wave }}(r, \phi, z)=\kappa(r, \phi, z) \times \delta(r, \phi)
$$

since a rise in density leads to an increase of the number of absorbers (unless for very opaque media, where radiation is absorbed at the very beginning of a considered volume element). The spiral arms can be described as adiabatic shock waves of an ideal gas, hence the adiabatic equation

$$
T P^{\frac{1}{\gamma}-1}=\text { const }
$$




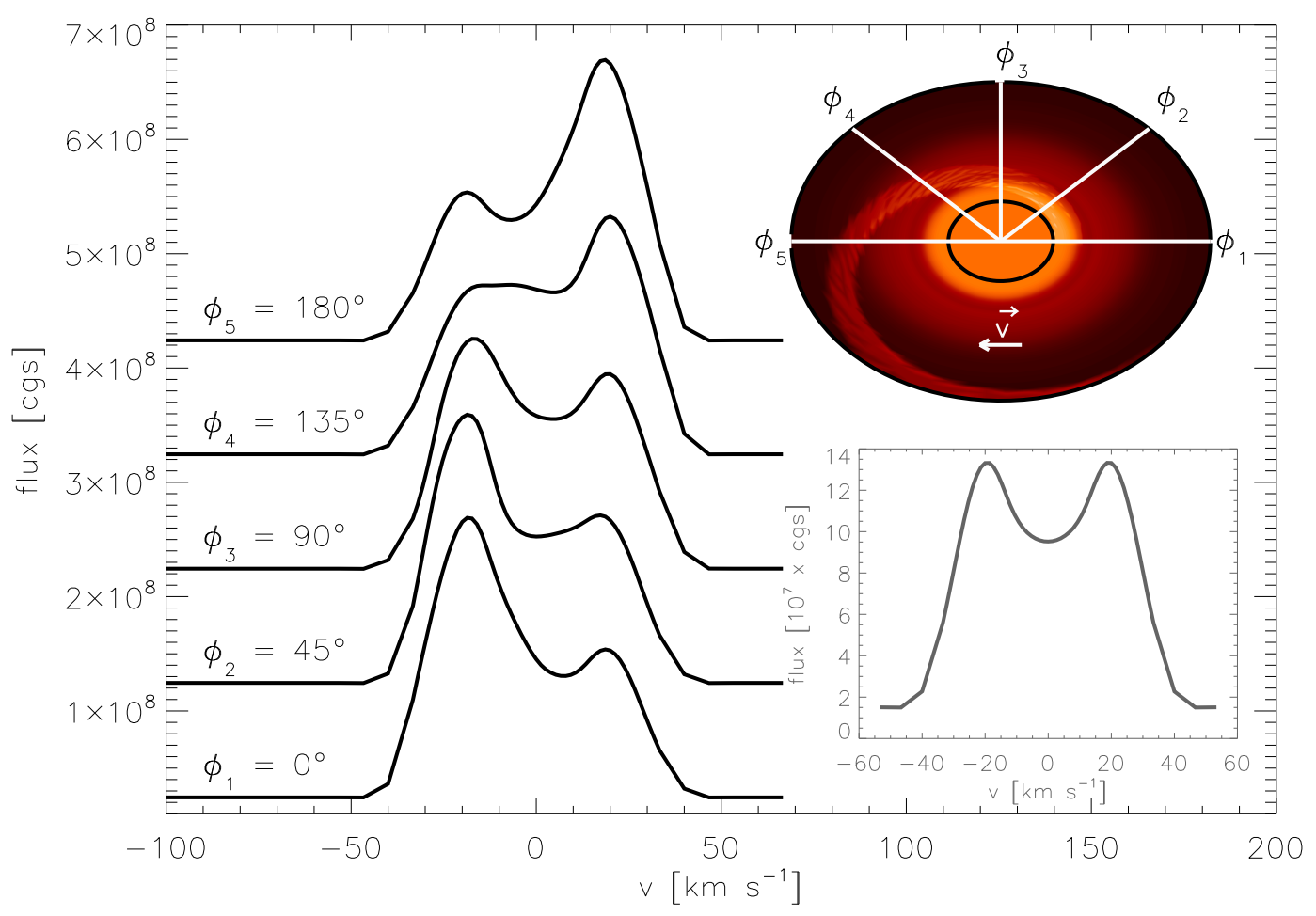

Figure 4.11: Line profiles for a disk with a density wave for different azimuthal viewing angles $\phi$ at constant inclination $i=20^{\circ}$. The opacity structure is plotted in the upper right corner together with the viewing direction $\phi_{1}-\phi_{5}$ and the Keplerian rotation direction $\vec{v}$ of the disk. The lines are offset in flux for clarity. The inset plot on the bottom right shows the line profile for the undisturbed disk as a reference.

and the ideal gas equation

$$
P=n k_{\mathrm{B}} T
$$

where $P$ is the gas pressure and $n$ the particle number density, lead to the relation

$$
T n^{1-\gamma}=\text { const } .
$$

As the diatomic molecule $\mathrm{H}_{2}$ is the most abundant species in cool circumstellar disks, the adiabatic exponent can be approximated to be $\gamma=1.4$. A change in density $\delta$ then results in a variation of the temperature

$$
T_{\text {wave }}(r, \phi, z)=T(r, \phi, z) \times \delta^{\alpha}
$$

with $\alpha=0.4$. This qualitative behaviour of temperature $T$ with a change in density $\delta$ is confirmed by radiative-hydrodynamical (RHD) simulations (Klev \& Crida, 2008; Klev et al., 2009). It should be noted that one would expect the adiabatic assumption to provide an upper limit for the temperatures corrections because in reality, especially in the less optically thick regions, there will be at least some energy exchange. However, fits for different exponents $\alpha$ in Eq. (4.31) to example radial surface density and temperature distributions from RHD models for a disk with 

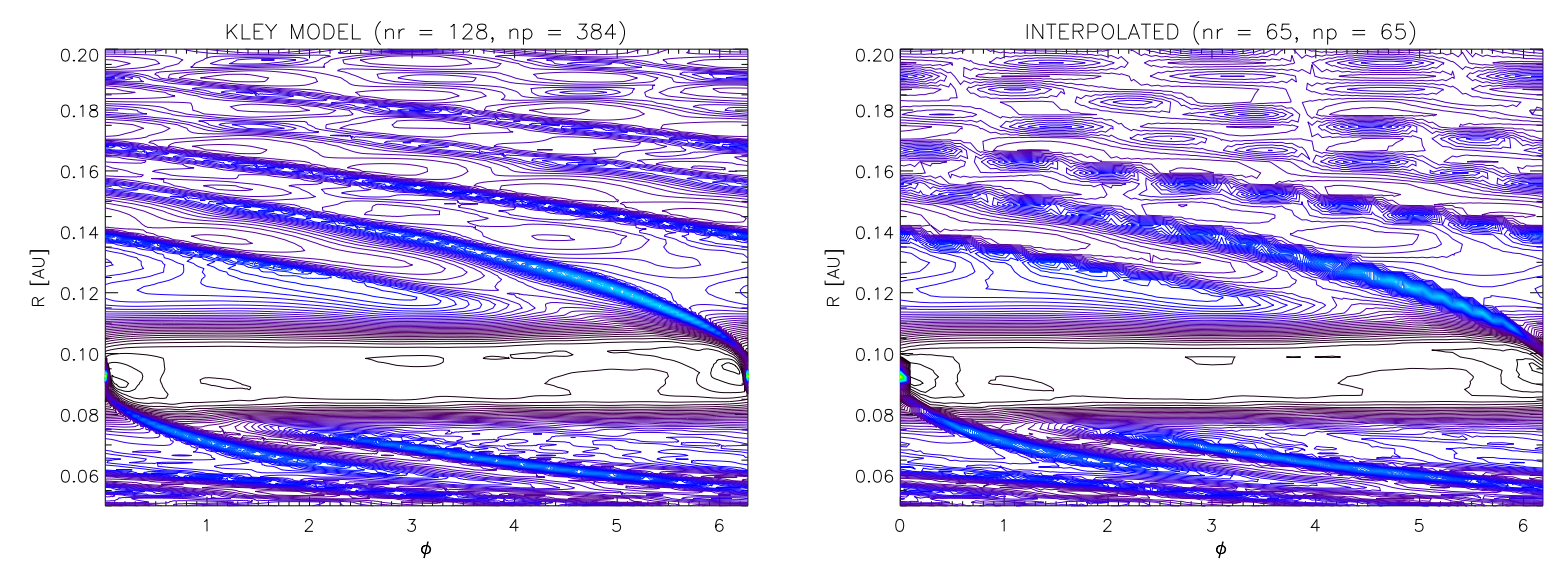

Figure 4.12: Density contour plots for a disk with embedded planet. The left plot shows the normalised surface density $\Sigma(r, \phi)$ calculated by W. Kley. The original structure has been scaled in $r$ according to the model setup used for the 3D line calculations. The right plot shows the same density structure interpolated on a $65 \times 65$ grid.

an embedded planet shown in Fig. 4.14 yield a slightly larger best-fit value than $\alpha=0.4$. The fit best reproduces the inner region of the disk and the gap but lacks accuracy in the outer region where the temperature is underestimated compared to the value determined in the disk-planet simulations.

Figure 4.11 shows the results for a 3DRT line transfer simulation with an increase in surface density in the wave of $\delta=1.5$ and an temperature modification exponent of $\alpha=0.4$. The azimuthally-averaged density plots shown in Fig. 4.13 support this choice of $\delta$. The line profile is shown for five different viewing angles of $\phi=0^{\circ}, 45^{\circ}, 90^{\circ}, 135^{\circ}$, and $180^{\circ}$ for a constant inclination of $i=20^{\circ}$. The disk opacity structure is also depicted together with viewing angles and Kepler rotation direction. Due to the increase of opacity and temperature in the density wave, this region emits stronger than the ambient unmodified disk. Hence, the additional flux in the blue wing of the line profile for $\phi=0^{\circ}$ compared to the profile of the undisturbed disk (bottom right inset in Fig. 4.11) can be directly attributed to the density wave in the inner region of the disk. The flux in the blue-shifted high-velocity part of the line profile is increased by a factor of $\sim 2$, a gain in flux which should be clearly detectable by modern high-resolution spectrographs. The line profile is affected when changing the azimuthal viewing angle $\phi$. Generally speaking, the line flux increases the strongest at the projected velocity $v$ which corresponds to the region where the density wave occupies the largest area between $R_{\text {in }}=0.05 \mathrm{AU}$ and $R \approx 0.12 \mathrm{AU}$ (the region which contributes most to the line; see Figs. 4.8 and A.1).

\section{Disk-planet models}

For the publication of de Val-Borro et al. (2006), the authors have performed test calculations with different codes for a setup of a Jupiter mass planet on a circular orbit which opens a gap in a protoplanetary disk during a few hundred orbital periods. In order to obtain a reasonable estimate for the opacity and temperature structure of a viscous disk with an embedded Jupiter mass planet, the density results of Kley 1 are used to modify the values of an undisturbed disk according to Eqs. (4.27) and (4.31).

${ }^{1}$ http://www.tat.physik.uni-tuebingen.de/^kley/projects/euplanet/ 


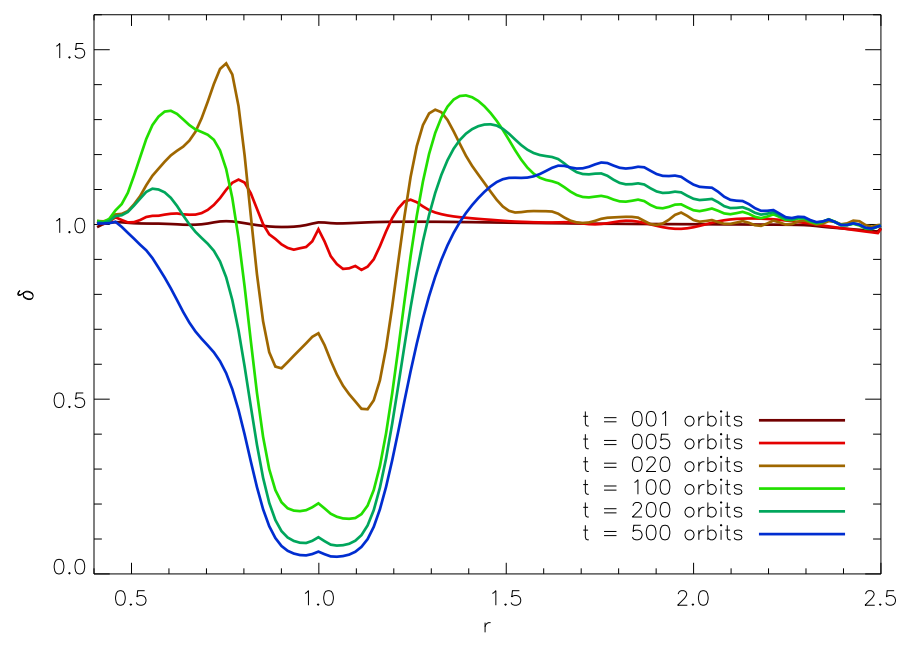

Figure 4.13: Run of the azimuthally-averaged surface density ratios $\delta$ with radius for a viscous disk with embedded Jupiter mass planet at different orbital periods.
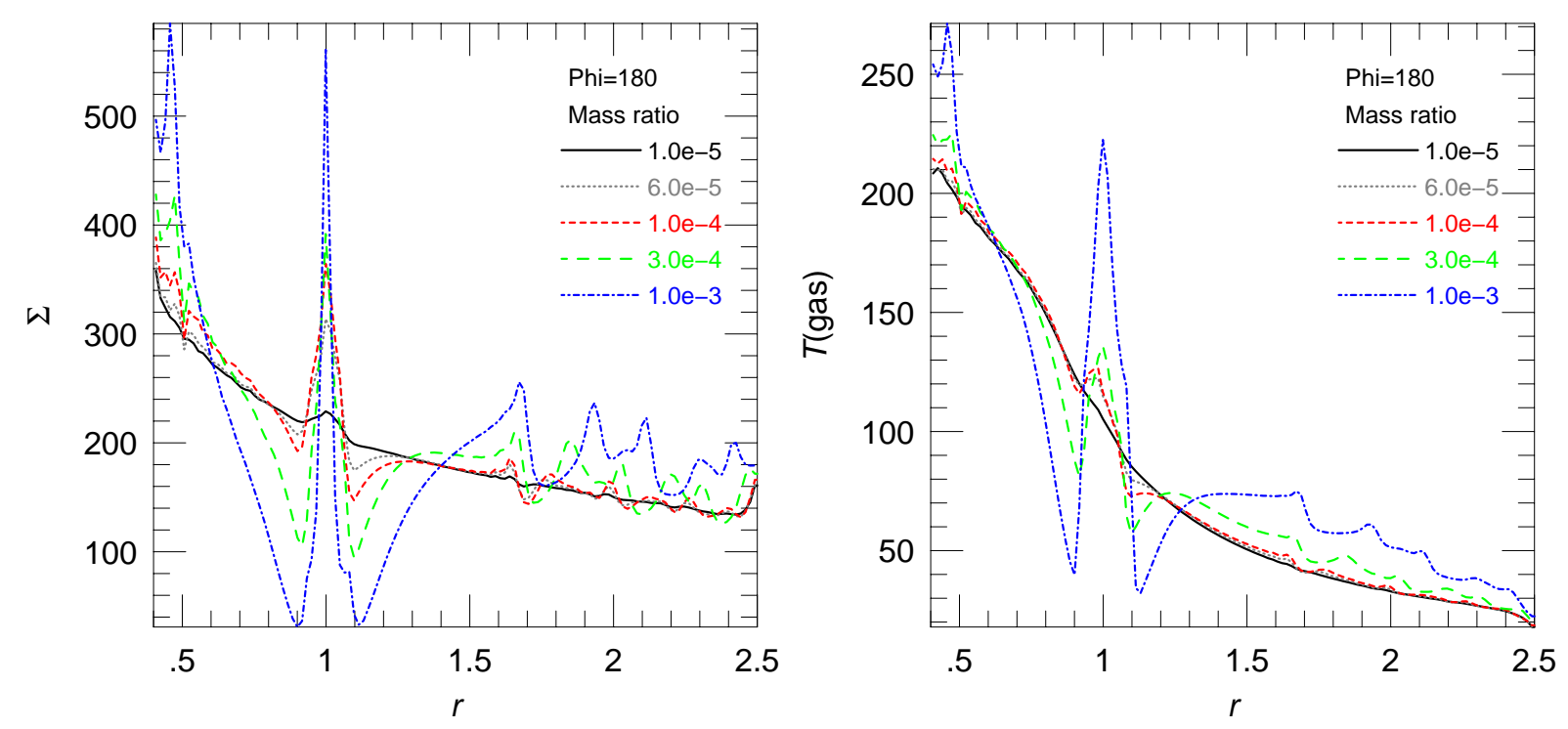

Figure 4.14: Radial surface density (left) and temperature structure (right) cut through a disk at the position of an embedded planet for different planet-disk mass ratios (kindly provided by W. Kley).

The original density structure of Kley (for 100 orbits) with $128 \times 384$ grid points and the one interpolated on the 3DRT grid with $65 \times 65$ grid points are shown in Fig. 4.12, Originally, the calculations were performed for a Jupiter at $R_{J}=5.2 \mathrm{AU}$ but they are here rescaled to the dimensions of the 3D model grid between 0.05 AU and 0.20 AU. Since the radius of the planet is scaled likewise, the planet only has a fourth of the radius of the Earth. In the following, models at two different evolutionary stages $(t=5$ and 50 orbits, see Fig. 4.13 for azimuthally-averaged $\delta$ plots) are used as input for line transfer modelling. 

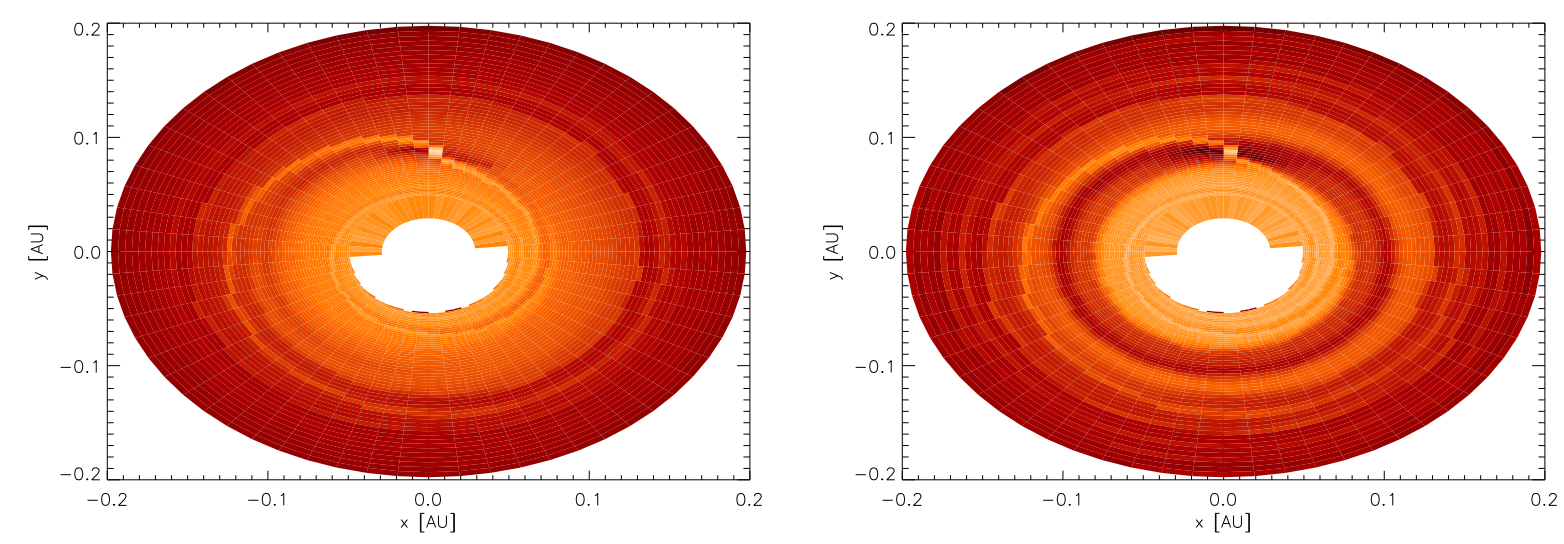

Figure 4.15: Continuum intensity plots for disk-planet models at $t=5$ orbits (left) and $t=50$ orbits (right). The inclination is $i=20^{\circ}$. The arc-like structure near the center of each plots is the intensity originating from the inner disk wall.

Model after 5 orbits: At this early evolutionary stage the gap formation due to the clearing of the protoplanet is not very advanced yet (see Figs. 4.13 and 4.15). The spiral arms have not formed as clearly as for instance in the $t=100$ orbits model shown in Fig. 4.12. The density increase near the planet is stronger than at later orbits though. The top plot in Fig. 4.16] shows the line profiles produced by the disk with planet at $t=5$ orbits together with the opacity structure and the undisturbed line profile. A clear deformation of the line profile compared to the one of the axisymmetric disk model is present for all viewing angles $\phi$ shown. When looking at the line contributions from different annuli (see Fig. A.2) it becomes clear that the flux increase in the blue peak of the $\phi=0^{\circ}$ line can be attributed to the spiral arm inwards of the planet. The central dip of the double-peaked line profile is filled by emission from the dense region around the planet. For the viewing angles $\phi_{1}-\phi_{3}$ the planet and the spiral arms around it are moving towards the observer causing an increase in line flux in the blue wing of the line. For $\phi_{5}=180^{\circ}$ most of the dense wave material inwards of the planet moves away from the observer causing the raise of flux in the red with an additional increase caused by the planet around $v \approx 0 \mathrm{~km} \mathrm{~s}^{-1}$. It should be noted at this point that the planet is slightly offset the $\phi=180^{\circ}$ position which causes a small shift of the planetary flux contribution in the line profile for $\phi_{1}$ and $\phi_{5}$. In this model approach (adiabatic density changes and planet in the very inner region of the disk) the alteration over the undisturbed disk is clearly visible in the theoretical line profile - especially in direct comparison between axisymmetric and non-axisymmetric disk line profiles. However, observations, e.g. those of RNO 90 presented in Sect. 3.3. show that the emitted line profiles from real disks deviate from simple axisymmetric accretion disk models. Whether these changes are caused by turbulent motion of matter in the disk or by an embedded planet (of the size used for the simulations here) that causes spiral arms is not unambiguously determinable.

Model after 50 orbits: At this stage gap clearing by the planet has progressed substantially leaving only weakly emitting matter in the annulus which includes the planet (see the azimuthallyaveraged density plots for $t=20$ and $t=100$ orbits in Fig. 4.13 as reference). The line profiles shown in Fig. 4.16 (bottom plot) in fact are quite symmetric. A look at the line profiles from the different regions in the disk (Fig. A.3) show that the flux from the planet annulus between $R=0.08-0.11 \mathrm{AU}$ is about a factor of 3 lower than in the $t=5$ orbits model, which explains 

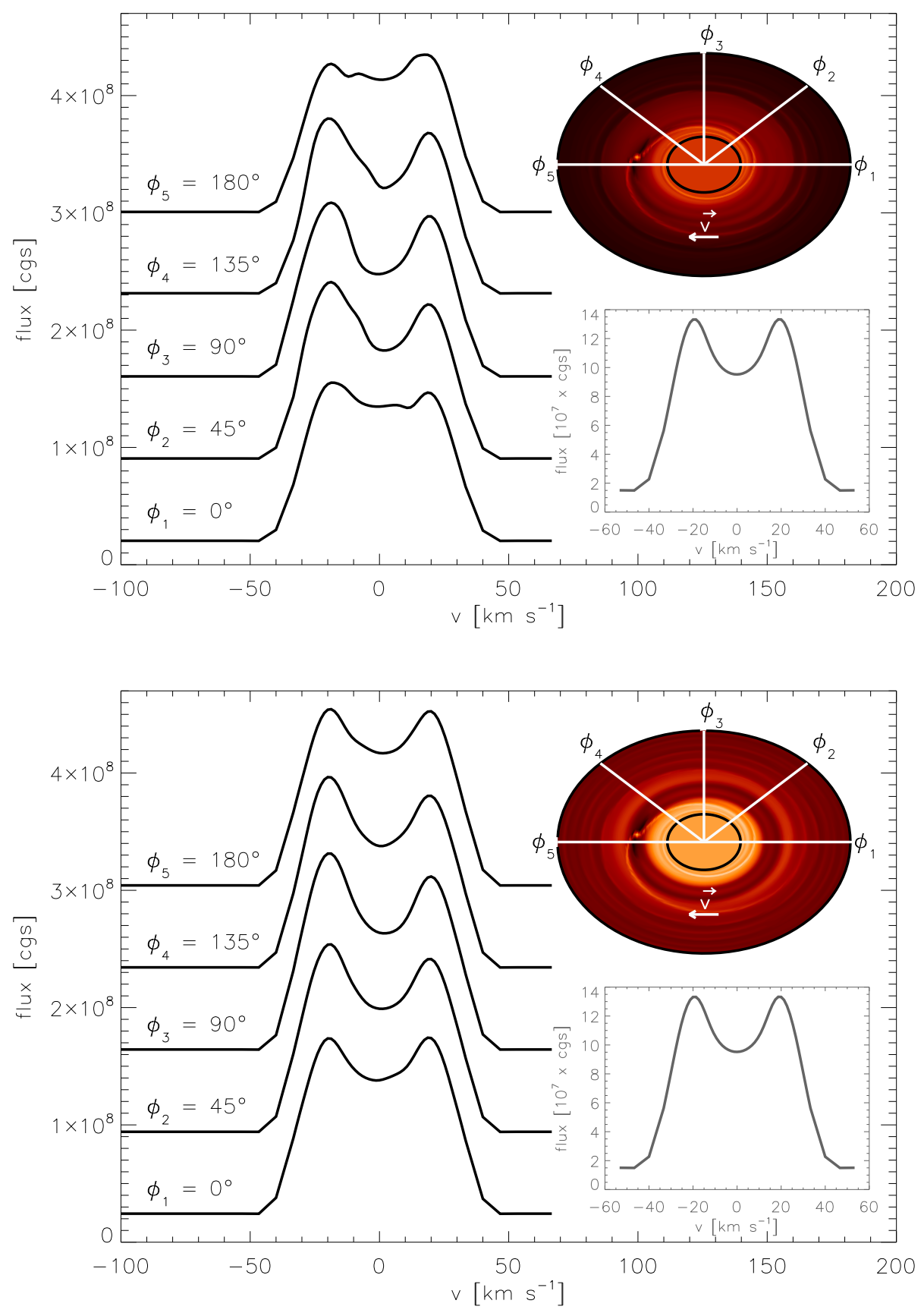

Figure 4.16: Line profiles for a disk harbouring a planet at $t=5$ orbits (top) and $t=50$ orbits (bottom) for different azimuthal viewing angles $\phi$ at constant inclination $i=20^{\circ}$. The opacity structure is plotted in the upper right corner together with the viewing directions $\phi_{1}-\phi_{5}$ and the Keplerian rotation direction $\vec{v}$ of the disk. The lines are offset in flux for clarity. The inset plot on the bottom right shows the line profile for the undisturbed disk as a reference. 
the missing flux excess near $v=0 \mathrm{~km} \mathrm{~s}^{-1}$ for the $\phi_{1}$ and the $\phi_{5}$ viewing angle as well as the magnification of the blue- and red-shifted peak caused by the spiral arm inwards of the planet, respectively. Overall, the changes in the line profile for this disk model over the axisymmetric one are small and only subtile in the model. A clear detection in spectroscopic observations seems unrealistic. How a larger, e.g. Earth- or even Jupiter-size, planet at these small orbital radii would influence the line profile needs to be studied in the future with detailed RHD models of the inner disk used as input for the 3DRT simulations. 


\subsection{Direct irradiation effects}

\subsubsection{Model approach}

In the case of $1+1 \mathrm{D}$ disk model atmospheres the central star is only irradiating the topmost layer of the atmosphere. Furthermore, radiation is not passed on in the radial direction. However, if there is an inner edge of the disk (at the inner disk truncation radius) caused e.g. by accretion of matter onto the star via stellar poloidal magnetic field lines which interact with the disk, the star will directly irradiate the inner edge almost at right angle over the whole height. Hence the material in this inner region will be hotter than that at larger radii and the very inner rim is expected to be hydrostatically "puffed-up" (for an overview of this effect see Dullemond et al., 2007). In order to model this effect 3D (or 2D) radiative transfer coupled with a temperature correction, a hydrostatic equilibrium determination, and a new calculation of the opacities is necessary (see Fig. 4.18). Since a frequency dependent RT with the opacity sampling method $\left(\sim 10^{6}\right.$ frequency points) used in the $1 \mathrm{D}$ case is not manageable in the $3 \mathrm{D}$ case in a reasonable amount of computing time, the frequency points have to be binned.

Different approaches have been used in the literature. The most essential simplification is to calculate an opacity or extinction mean over the entire frequency range (Mihalas, 1978). If one wishes to obtain the correct value for the integrated energy flux from the second moment of the RTE $\partial K_{\nu} / \partial z=-\chi_{\nu} H_{\nu}$, one easily sees that this can be done by

$$
\bar{\chi}^{-1}=\int_{0}^{\infty} \frac{1}{\chi_{\nu}} \frac{\partial K_{\nu}}{\partial z} d \nu / \int_{0}^{\infty} \frac{\partial K_{\nu}}{\partial z} d \nu
$$

Since $K_{\nu}$ is not known before the solution of the RTE, an approximation for large optical depth $(\tau \gg 1)$ is used, i.e. $K_{\nu} \rightarrow \frac{1}{3} J_{\nu}$ and $J_{\nu} \rightarrow B_{\nu}$. Hence, using the approximation $\partial K_{\nu} / \partial z \approx$ $\frac{1}{3}\left(\partial B_{\nu} / \partial T\right)(d T / d z)$ leads to the mean extinction called the Rosseland mean which reads

$$
\bar{\chi}_{R}^{-1}=\int_{0}^{\infty} \frac{1}{\chi_{\nu}} \frac{d B_{\nu}}{d T} d \nu / \int_{0}^{\infty} \frac{d B_{\nu}}{d T} d \nu
$$

The Rosseland mean $\left(\bar{\kappa}_{R}\right.$ from here on) is a harmonic mean and, therefore, gives the highest weight to those regions where the opacity is the lowest and the greatest amount of radiation is transported. The Rosseland mean is not a valid approximation in optically thin regions, i.e. parts of the atmosphere where line formation occurs. A different approach for an opacity mean is to demand that it yields the correct integrated thermal emission value. This opacity mean is called the Planck mean and is obtained by

$$
\kappa_{B}=\int_{0}^{\infty} \kappa_{\nu} B_{\nu} d \nu / \int_{0}^{\infty} B_{\nu} d \nu
$$

This mean is the best choice if radiative equilibrium near the atmosphere's surface is required.

Mihalas (1978) explains the concept of opacity distribution functions (ODF, see also Strom \& Kurucz, 1966) which replaces a detailed opacity spectrum. In this method, the spectrum is subdivided into several $\left(\sim 10^{2}\right)$ wavelength bins and for each bin, the fraction of frequency points above a list $(\sim 10)$ of opacity thresholds is calculated. This procedure can be repeated for a mesh of temperature and pressure values before the actual RT calculations. Hence, one will reduce the determination of the frequency dependent opacities and the number of frequency points by a factor of $\sim 10^{3}$ for a $10^{6}$ frequency point spectrum. This method demands some preparations prior to the calculations. It also assumes that the position of the lines in frequency does not change 


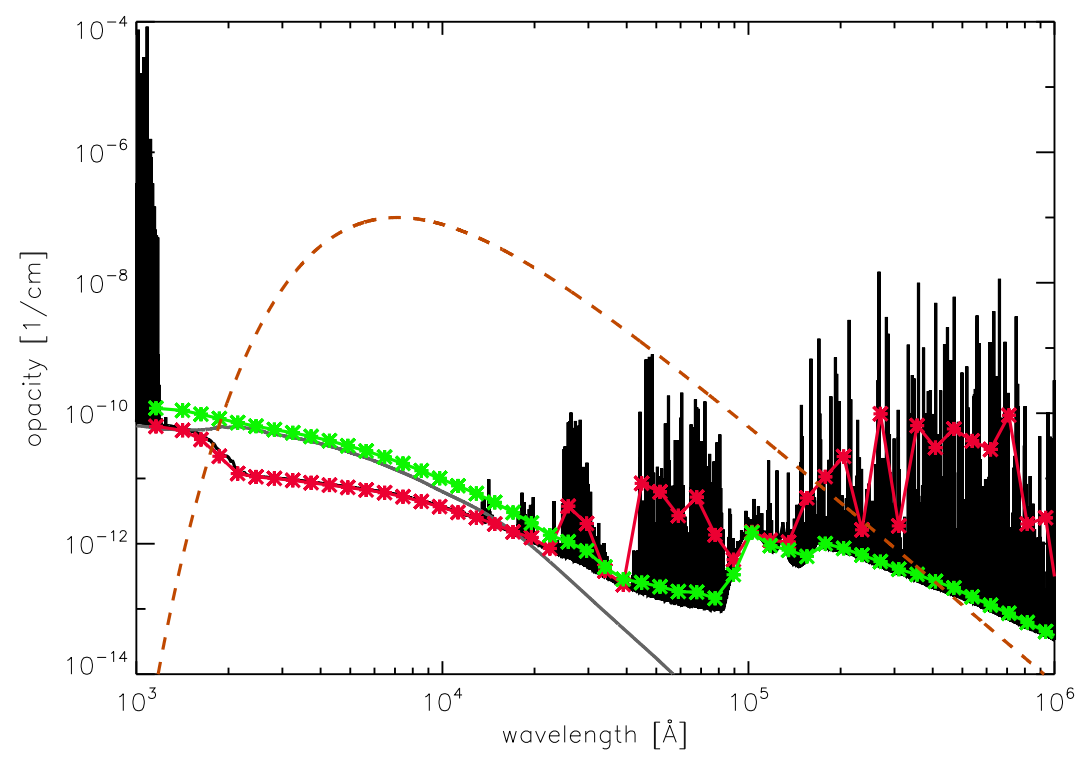

Figure 4.17: Run of the absorption coefficient $\kappa_{\lambda}$ (black line) and scattering coefficient $\sigma_{\lambda}$ (grey line) with wavelength for an upper layer in a 1D disk atmosphere at $R=$ $0.048 \mathrm{AU}$. The red line is the Planck mean opacity $\kappa_{B}$ and the green line the Rosseland mean extinction $\chi_{R}$ for 50 frequency bins. The dasehd brown line represents a $4000 \mathrm{~K}$ blackbody spectrum in arbitrary units.

from layer to layer and that the threshold bins are equally well chosen for all layers (that is not always the case).

The mulitgroup approach was first proposed by Nordlund (1982) and suggests the division of the spectrum into a small number (typically 3-5) of subsets $\Omega_{i}$ according to the strength of the corresponding opacities. This way continuum and line opacities of different magnitude are grouped and bin-averaged opacity means have to be calculated to solve the RTE. Ludwig (1992) introduced a hybrid scheme according to

$$
\bar{\kappa}_{i}=2^{-\frac{\tau_{i}}{\tau_{0}}} \bar{\kappa}_{B, i}+\left(1-2^{-\frac{\tau_{i}}{\tau_{0}}}\right) \bar{\kappa}_{R, i}
$$

with the threshold optical depth $\tau_{0}=0.35$ and the bin-averaged means $\bar{\kappa}_{B, i}$ and $\bar{\kappa}_{R, i}$ according to Eqs. (4.34) and (4.33), respectively. The mean opacity $\bar{\kappa}_{i}$ can be tabulated for different $(P, T)$ values. One problem with this method is that the optical depth $\tau_{i}$ as a function of $(P, T)$ is not known a-priori and has to be estimated and secondly, the sorting of opacities into bins has to be done in a way that the choice of bins is as independent of $(P, T)$ as possible (see Vögler et al., 2004, for a discussion of different methods).

For the calculations presented here, a less elaborate approach was chosen in order to keep computational time low. The opacities are calculated for a set of $1 \mathrm{D}$ disk structures for $N_{1 \mathrm{D}}$ frequency points. The points are spaced equidistantly on a logarithmic wavelength grid between $\lambda_{\min }$ and $\lambda_{\max }$. For the calculations $N_{1 \mathrm{D}}=10^{5}$ and $\lambda_{\min }=10^{3} \AA$ and $\lambda_{\max }=10^{6} \AA$ were chosen to cover the region where the central star emits most of its flux up to the region where thermal emission of the inner disk material can be neglected. Since an iterative technique between 1D hydrostatic and opacity calculations and 3D RT and temperature corrections is used, the number 


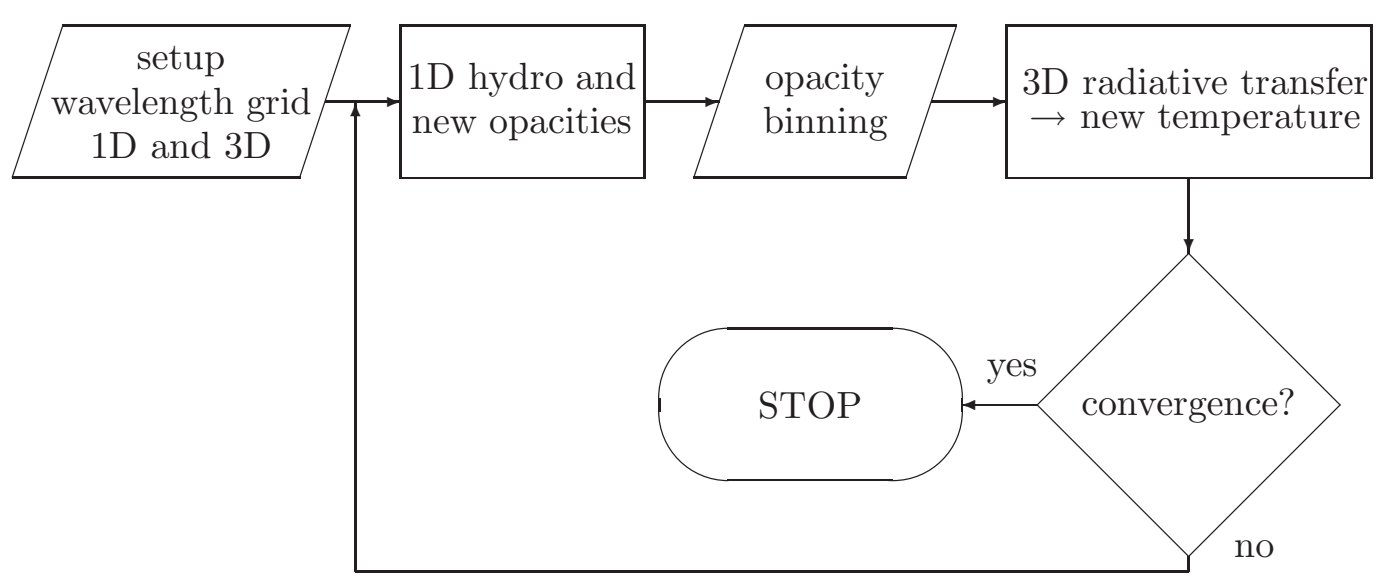

Figure 4.18: Flow chart of 3D structure calculations. First the wavelength grids for the $1 \mathrm{D}$ and 3D models are set up. Then the hydrostatic equation for a set of $1 \mathrm{D}$ models is solved and opacities for a large number of wavelength points are evaluated. In a next step, the opacities are binned and the RT is solved in 3D for a smaller number of wavelength points using the mean opacities. A temperature correction for each 3D grid point is performed and the new temperature structures are fed back into the 1D model structures. This procedure is repeated iteratively until convergence in temperature is achieved.

$N_{1 D}$ is deliberately kept small to save time during $1 \mathrm{D}$ structure and opacity calculations. Before the start of an iterative model run, the wavelength grids are setup once for $N_{1 \mathrm{D}}$ between $\lambda_{\min }$ and $\lambda_{\max }$ and the $N_{3 \mathrm{D}}$ (typically 10 points) wavelengths and wavelength bins are determined. The Rosseland and Planck opacities as well as mean thermalisation parameters $\bar{\epsilon}_{i}$ are then computed for each bin and $1 \mathrm{D}$ structure model. This way the mean opacities do not have to be calculated beforehand for a large set of $(P, T)$ values but only for the actual conditions in each layer of a set of $1 \mathrm{D}$ structure models. However, the binning is done on the frequency grid without the $\kappa$-sorting employed in the ODF and the multigroup approach (see Fig. 4.17 for binning results). As noted before, the Rosseland mean is preferable at large optical depths while in the optically thin surface layers the Planck mean is better suited. For the calculations shown here the Rosseland mean was chosen as the mean opacity since it is a better representation for the disk taken as a whole. A hybrid scheme as suggested by Ludwig (1992) is hard to realise because the knowledge of $\tau_{i}$ at a given point is necessary before calculating the $\bar{\kappa}_{i}$ which in turn is needed to determine the $\tau_{i}$. Knowing the mean opacities in each wavelength bin, the RT is solved with 3DRT and the calculated mean intensity $J=\sum_{i} \frac{1}{2}\left(J_{i}+J_{i+1}\right) \Delta \lambda_{i}$ is used to perform a local temperature correction at each grid point according to

$$
\Delta T=2^{-i_{\mathrm{d}}} \frac{\pi}{4 \sigma T^{3}}\left(\frac{\kappa_{J}}{\kappa_{B}} J-B\right)
$$

where $i_{\mathrm{d}}$ is a damping index, $i_{\mathrm{d}} \in \mathbb{N}^{+}$. The mean opacities $\kappa_{J}$ and $\kappa_{B}$ are read from the $1 \mathrm{D}$ restart files and interpolated on the $3 \mathrm{D}$ grid. The new temperature values at each grid point $\left(i_{r}, i_{\phi}, i_{z}\right)$ are then interpolated onto the original 1D structures using cubic polynomials and the updated 1D structures are saved in the restart files. These are taken as input for new 1D hydrostatic and opacity calculations thereupon. 

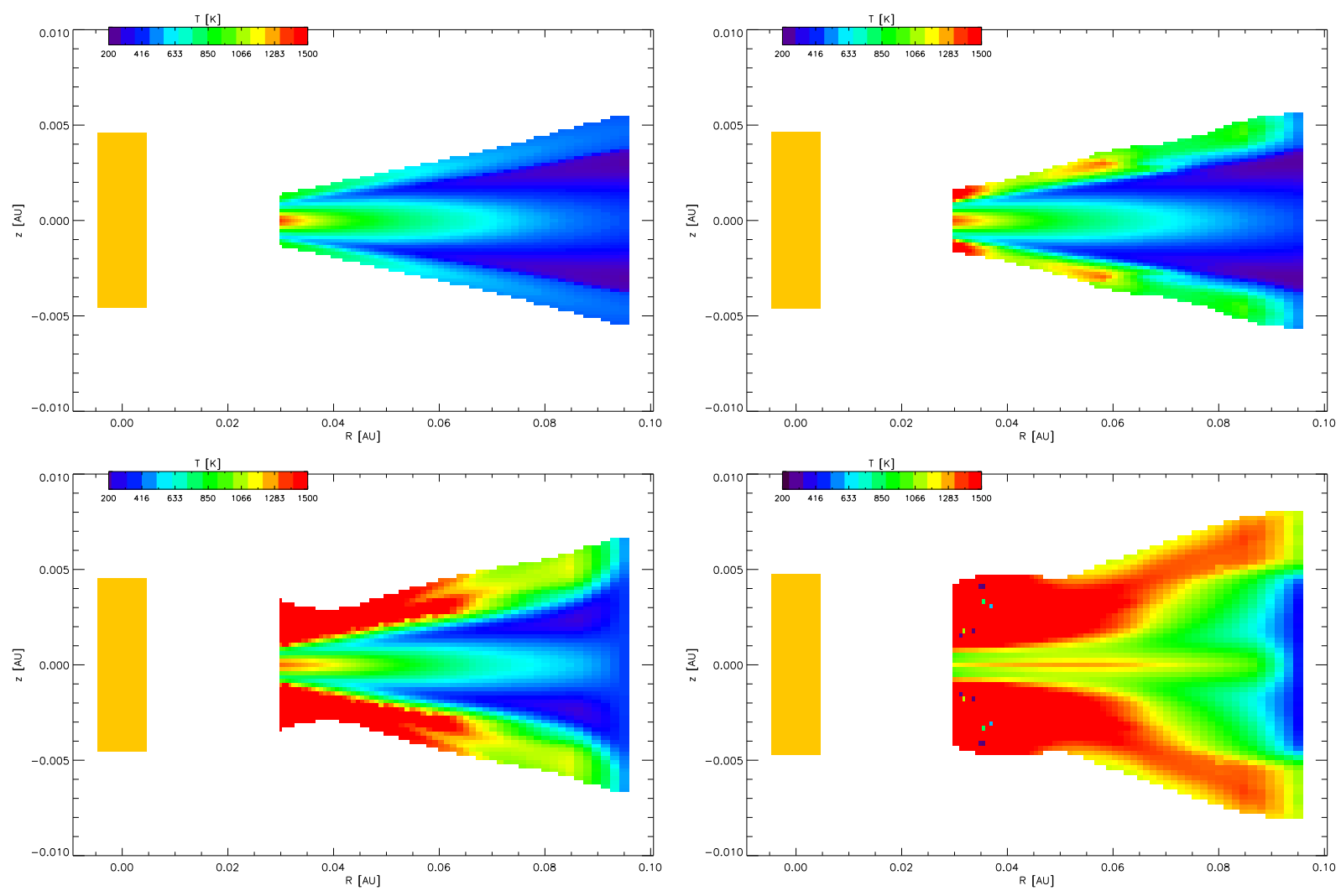

Figure 4.19: $3 \mathrm{D}$ temperature structures for a disk at different iteration steps: $1 \mathrm{D}$ input structure and results after 3, 10, and 50 iterations (left to right, top to bottom). The yellow bar at left of each panel represents the central star.

\subsubsection{Simulation results}

\section{Setup I}

A set of eight 1D disk structures with $\dot{M}=3 \times 10^{-9} M_{\odot} \mathrm{yr}^{-1}, R e=5 \times 10^{4}, R_{\star}=1 R_{\odot}$, $M_{\star}=0.8 M_{\odot}, T_{\text {eff }}=4000 \mathrm{~K}, R_{\text {in }}=0.03 \mathrm{AU}$, and $R_{\text {out }}=0.10 \mathrm{AU}$ was used to investigate the effect of direct irradiation on the structure of the very inner disk including gas opacities. The disk is assumed to be in LTE and effects such as photoevaporation by stellar X-rays, EUV, and FUV photons analysed e.g. by Woitke et al. (2009) are neglected. The simulation was run for 50 iterations using the harmonic Rosseland mean in 50 opacity bins. This description of the absorptivity takes into account that the radiation in the optically thick parts of the cool disks is mostly blocked by the large number of molecular lines and hence the radiation is transported in the opacity gaps (Muzerolle et al., 2004). In less optically thick regions $(\Delta \tau<1)$ the absorptivity is underestimated and the Planck opacity would be better suited. A switch between the different opacity binning methods would be the appropriate choice. A problem here is the determination of $\Delta \tau$ because the optical depth itself depends on the choice of opacity description, hence, only the Rosseland mean is used. In order to save a factor of $\sim 10$ in computing time, scattering was not accounted for in the 3D simulations which is justified by the small scattering fraction found in the disk models $(\epsilon>0.8)$. As given in the definition of the Rosseland mean, the scattering fraction $\sigma$ is included in the opacity, i.e. $\chi=\kappa+\sigma$. The grid was chosen to have $65^{3}$ points and 


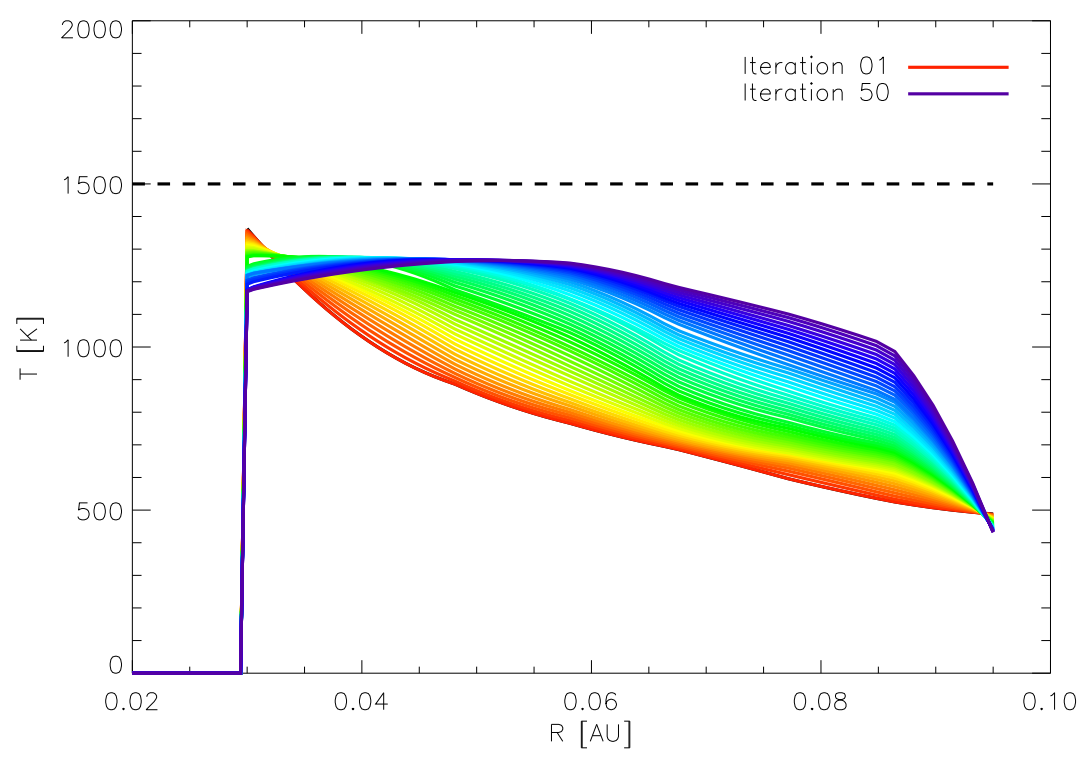

Figure 4.20: Midplane temperature history for disk setup I. After 50 iterations the midplane temperature is below the dust destruction temperature at all radii. Close to the star the temperature is even reduced compared to the $1 \mathrm{D}$ value despite the direct irradiation taken into account in the $3 \mathrm{D}$ radiative transfer.

$32^{2}$ solid angle points. Dust was included in the opacity structures from the first iteration on and the number of wavelength bins was set to 50 (see Fig. 4.17 for wavelength coverage). This setup requires $\sim 3 \mathrm{CPU}$ hours of computation time per iteration on a Mac Pro with two $2.66 \mathrm{GHz}$ Dual-Core Intel Xeon processors in a MPI-parallelised version of 3DRT run on all 4 CPUs.

Figure 4.19] shows the original $1 \mathrm{D}$ temperatures interpolated on the $3 \mathrm{D}$ grid as well as the temperature structures after 3,10 , and 50 iterations. One would expect that the complete inner wall that is directly irradiated by the central star is heated up and becomes isothermal. However, due to the finite resolution of the grid, the optical depth step $\Delta \tau$ near the midplane is so large that the stellar radiation does not "reach" the innermost grid point. Therefore, only the less optically thick upper layers receive direct stellar irradiation, which they did not in the $1+1 \mathrm{D}$ model approach, and the temperature rises. The temperature increase in the outer layers leads to an hydrostatic expansion of the disk atmosphere which is especially strong close to the star. The disk height in the inner disk region seems to converge to $z_{\max } \approx R_{\star}$ for iteration 30 and after. Generally, the disk vertically expands compared to the $1 \mathrm{D}$ models by a factor of $\sim 1.7$. At radii $R \gtrsim 0.035 \mathrm{AU}$ the midplane temperature increases because here the optical depth is smaller and more radiation from surrounding layers can reach down to $z=0$. At radii $R \lesssim 0.05 \mathrm{AU}$ the temperature decreases after $\sim 25$ iterations, caused either by the neglected viscous heating in the temperature correction scheme (see Eq. (4.36)) or by the open boundary at the inner disk wall. The radial component of the flux vector $F_{r}\left(R_{\text {in }}, \varphi, 0\right)$ points inwards supporting the latter explanation. The temperature structure plots in Fig. 4.19 show that the heating of the disk, also in the regions very close to the star, numerically diffuses towards the midplane. In principle it is possible that the $\Lambda$-iteration-like temperature correction scheme used here never converges towards the "true" temperature value. In fact, the run of the maximal $T$-correction in the midplane $\left(\frac{\Delta T}{T}\right)_{\max }$ with iteration number drops off very slowly. If it does eventually converge, 


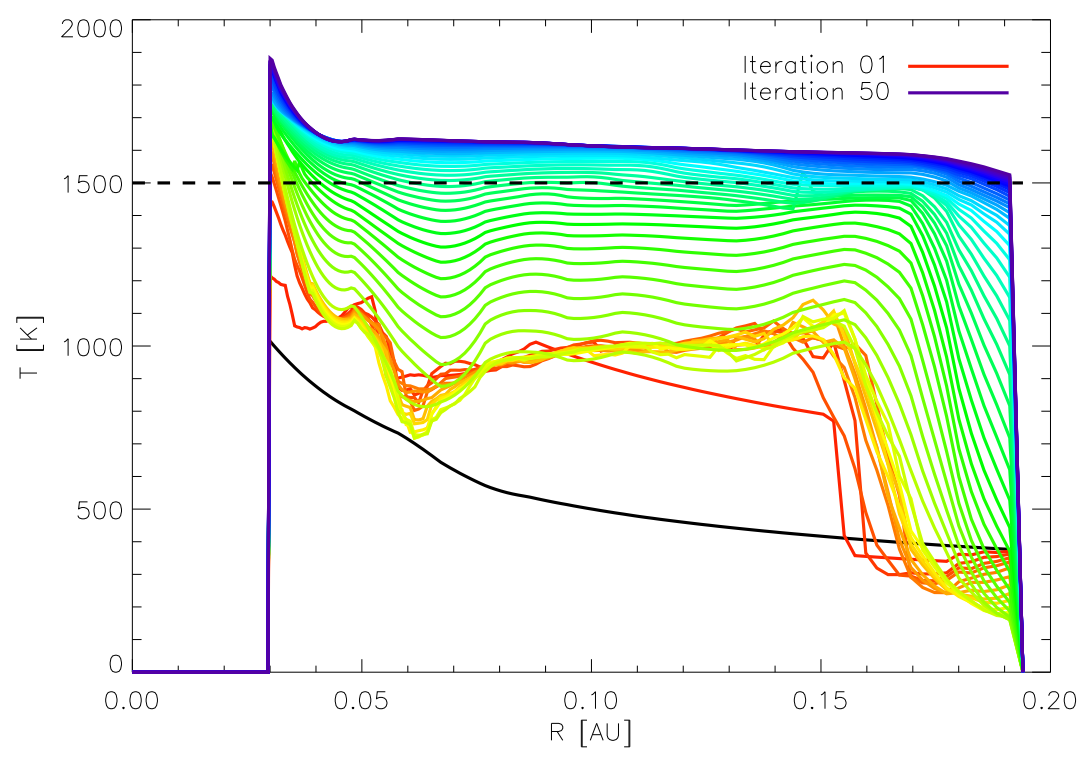

Figure 4.21: Midplane temperature history for disk setup II. The first 13 iterations only gas is considered as opacity source, then dust grains with $a_{0}=6.25 \times 10^{-1} \mu \mathrm{m}$, $a_{0}=6.25 \times 10^{-2} \mu \mathrm{m}$, and $a_{0}=6.25 \times 10^{-3} \mu \mathrm{m}$ base size were included for 19,4 , and 4 iterations, respectively.

another $\sim 100$ iterations would probably be necessary.

Nevertheless, it can be learned from this setup that the convergence behaviour of the iteration scheme presented in Eq. (4.36) is slow and either an iterative acceleration procedure needs to be implemented or a different approach for a faster (shorter CPU time) convergence towards the "true" temperature has to be found. In setup II such an alternative strategy is presented.

\section{Setup II}

The disk setup presented in this Section differs from the disk model setup I described above in three aspects. First, in order to reduce computing time, the number of wavelength bins in 3DRT was reduced from 50 to 5 saving a factor of 10 but at the cost of a less accurate irradiation and opacity treatment. A comparison to calculations with the same disk setup but for 50 wavelength points shows that the total stellar irradiation is overestimated by $\sim 25 \%$. Furthermore, the mean opacities are binned in large wavelength chunks which leads to a less accurate opacity treatment. However, the differences between the calculations are not substantial (e.g. a star with a temperature of $T_{\text {eff }}=4200 \mathrm{~K}$ would have the same luminosity as the poorly sampled $T_{\text {eff }}=$ $4000 \mathrm{~K}$ star used here) and a qualitative analysis of the $3 \mathrm{D}$ radiative transfer and temperature determination can be achieved despite the approximations. Second, the opacity is restricted to gas and neglects dust contributions in the first 13 iterations. This way the optical depth steps $\Delta \tau$ are significantly reduced and the stellar irradiation can penetrate deeper into the disk. Hence, the long-lasting temperature diffusion process described in setup I can be overcome. This strategy also includes that dust is successively considered (starting with large dust grains which contribute little to the opacity and decreasing the dust grain base size $a_{0}$ successively; see Sect. 3.1.5) once the "gas temperature" is converged. Consequently, dust will not be present as opacity source if 


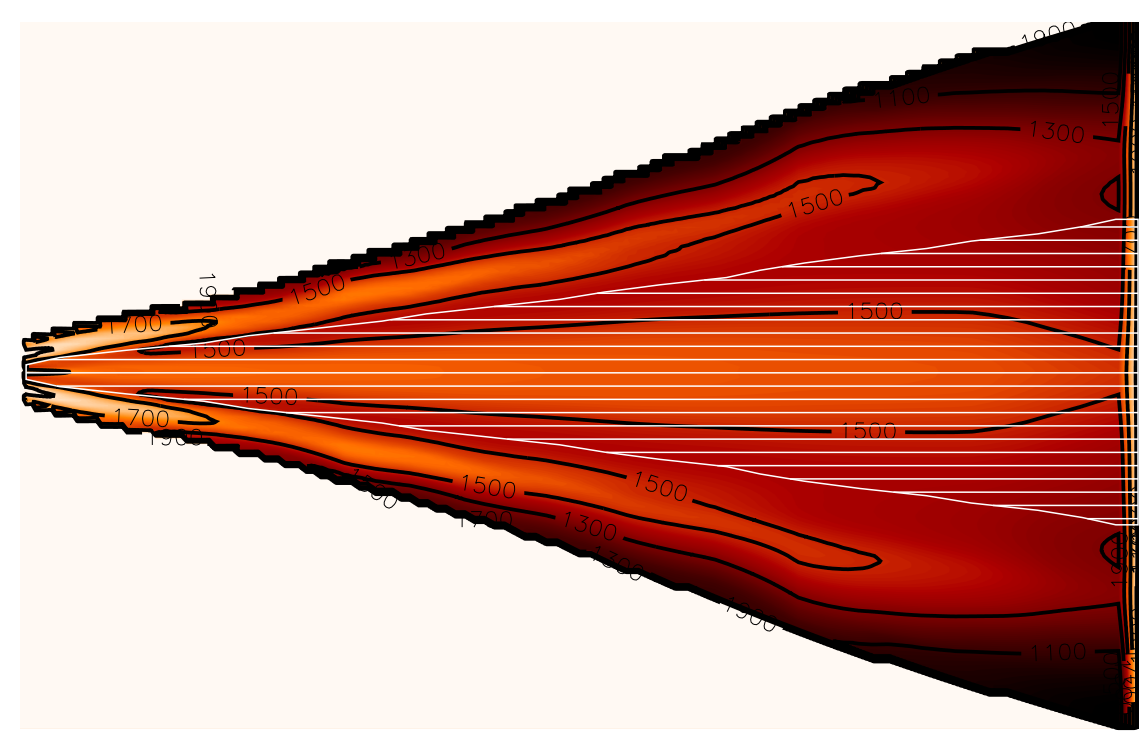

Figure 4.22: Temperature contour plot for disk setup II near convergence after 50 iterations. The very inner directly irradiated region, the midplane, and the outer layers exceed the dust evaporation temperature of $1500 \mathrm{~K}$. The white shaded area marks the region with $\tau>1$ in the radial direction.

temperatures exceed the typical value for silicate sublimation of $\sim 1500 \mathrm{~K}$. Third, the number of $1 \mathrm{D}$ models is increased to 16 extending the disk to $R_{\text {out }}=0.194 \mathrm{AU}$. In order to work with a similar grid resolution as in setup I, the number of grid points in radial and vertical direction is increased to 129 .

Figure 4.21 shows the iteration history of the midplane temperature. For the first 13 iterations only gas is considered as opacity source while dust is only included in the EOS. By adopting this approach the long-lasting heating of the optically thick midplane region can be avoided. Within the 13 iterative steps, the very inner part exceeds the dust evaporation temperature of $1500 \mathrm{~K}$, a value that the same region in setup I does not even reach after 50 iterations. Once iterative changes in the "gas temperature" are small, dust is switched on in the opacity calculation. First, only large grains with a base size of $a_{0}=6.25 \times 10^{-1} \mu \mathrm{m}$ are considered for 19 iterations. This approach guarantees that the opacity is not increased abruptly and that the heating of the disk proceeds smoothly. Then the grain base size is lowered to the ISM value $a_{0}=6.25 \times 10^{-3} \mu \mathrm{m}$ in two steps of 4 iterations each until the temperature structure converges. Further test calculations have shown that also a direct inclusion of ISM sized dust grains after the pure gas simulations leads to very similar temperature structure results. Moreover, the disk from setup II sampled with 5 wavelength bins shows a much faster heating process. Hence, the reduction of wavelength points from 50 to 5 is bought at the expense of a less accurate and less realistic RT treatment.

At iteration 50 the maximal temperature correction is below $1 \%$. The temperature correction at radii $R>0.170 \mathrm{AU}$ is damped in order to avoid blow-up processes of the disk at the outer boundary which have occurred in earlier calculations. By neglecting dust opacities in the first few iterations, the temperature in the disk, especially near the midplane, can be quickly increased. Switching on the dust at these increased temperatures, the temperature quickly rises and the dust completely evaporates at the inner disk rim, in the upper layers near the surface, and in the midplane (see Fig. 4.22). The synthetic temperature structure shows that the hottest regions of the upper layers are not at the very surface but slightly below which points at an opacity effect, 


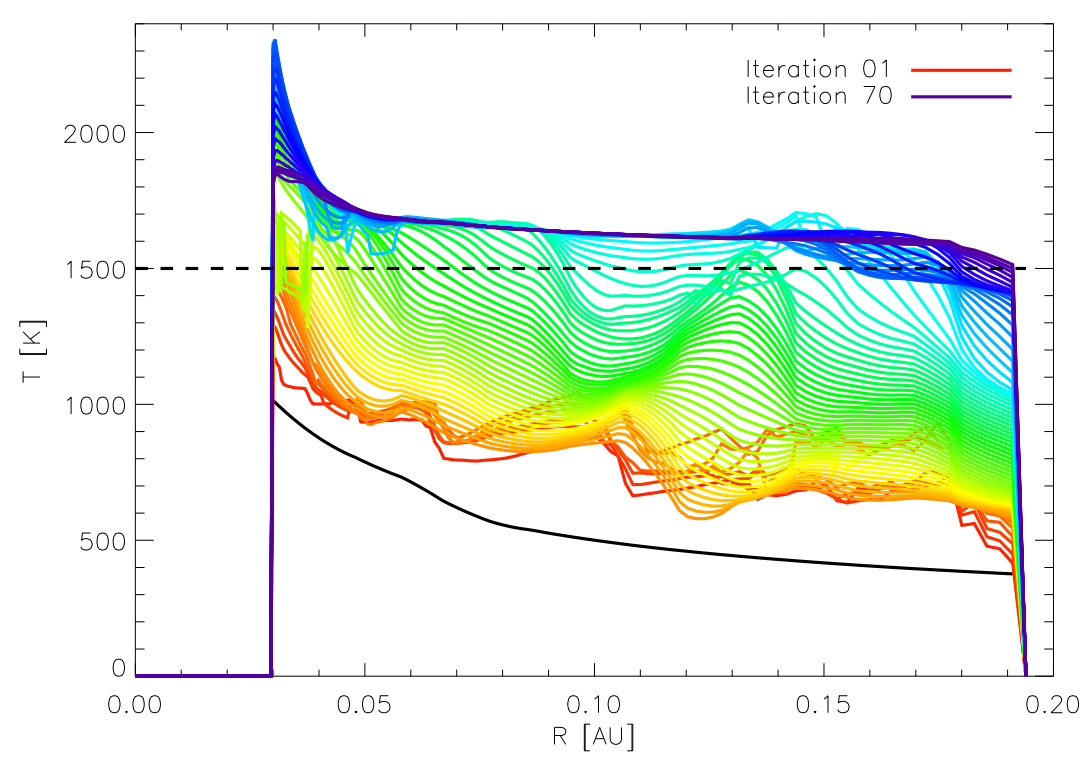

Figure 4.23: Midplane temperature history for disk setup III. The first 7 iterations only gas is considered as opacity source, then dust grains with $a_{0}=6.25 \times 10^{-3} \mu \mathrm{m}$ base size were included for a total of 70 iterations.

i.e. in the topmost optically thin regions the larger Planck opacity would be more appropriate than the Rosseland mean. Despite the fact that the complete midplane region has $T>1500 \mathrm{~K}$, and is hence devoid of dust, it does not become optically thin in this model. In fact, only a fraction of 0.58 of the disk height at $R=0.194 \mathrm{AU}$ is optically thin to stellar irradiation.

\section{Setup III}

This setup is similar to setup II but now 10 wavelength bins are used and the number of polar solid angle points is increased from $n_{\theta}=32$ to $n_{\theta}=64$, slowing down the computations by a factor of 4 . In this case, however, the wavelength coverage is much better and test calculations show that the results agree well with those for 50 wavelength points.

The first 7 iterations are calculated without dust opacities. After the dust is switched on as opacity source (directly with ISM grain base size), the temperature rises quickly and temperatures of up to $\sim 2200 \mathrm{~K}$ are reached in the very inner part of the disk near the midplane around iteration 50 (see Fig. 4.23). These high temperatures are not sustained and drop down to $\sim 1800 \mathrm{~K}$ in iteration 70. The inner disk region is not heated smoothly but the highest temperatures are reached near the midplane and in the top layers, as is the case in setup II. This effect could be a result of a poor solid angle point resolution. A further increase of solid angle points to verify this assumption is not manageable under the given circumstances, i.e. computing power and time. Alternatively, this non-smooth heating at the inner rim can be explained by the initial 1D structures which are hotter in the midplane (viscous heating) and top layers (irradiation heating) than in the intermediate layers of the atmosphere.

In comparison to setup II, which had only half of the number of wavelength bins and half the polar solid angle points, the disk in model setup III is heated more uniformly. The overall temperature structure is very similar for setup II and III (compare final midplane temperatures in 


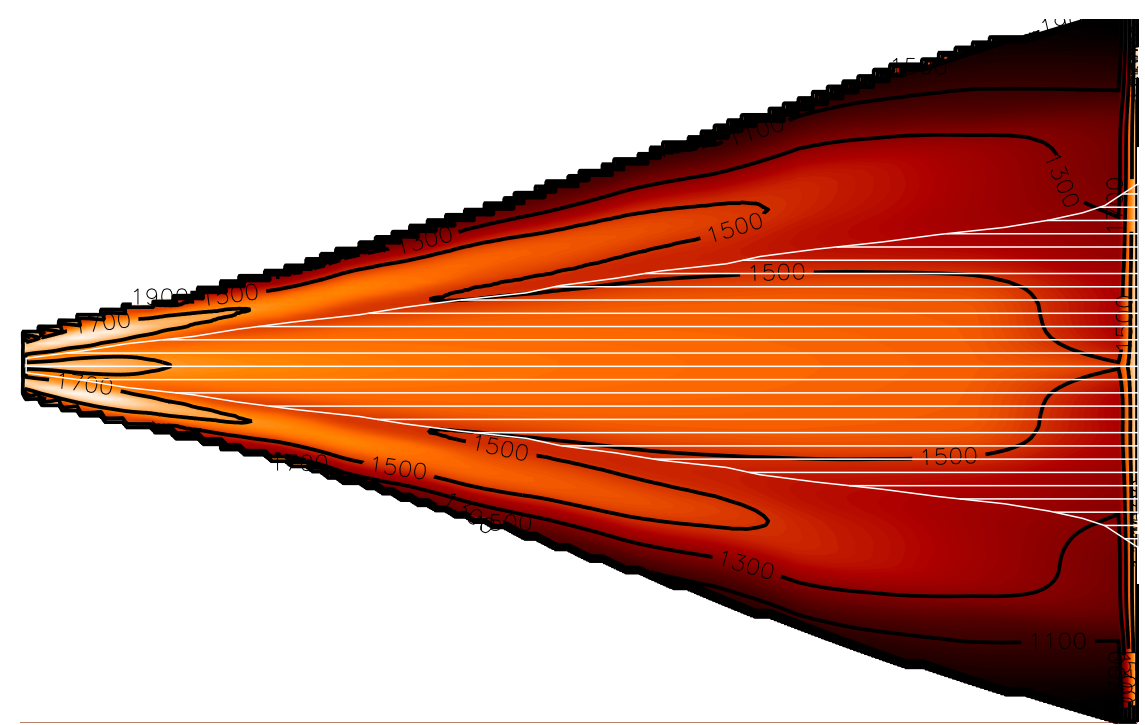

Figure 4.24: Temperature contour plot for disk setup III near convergence after 70 iterations. Due to the larger number of polar solid angle points compared to setup II, the inner region is heated more smoothly. The white shaded area marks the region with $\tau>1$ in the radial direction.

Figs. 4.21 and 4.23). The temperature increase in the midplane region $(T>1500 \mathrm{~K})$ is vertically more extended in setup III. The fraction of the disk height at $R=0.194 \mathrm{AU}$ where the disk is optically thin to stellar irradiation is comparable to setup III (see white shaded area in Figs. 4.22 and 4.24$)$.

\subsubsection{Discussion}

The direct irradiation calculations presented in this Section should provide a first study of how important the radial radiative transfer is on the disk structure. Models for three disk setups were simulated showing similar overall behaviours. The different wavelength bin and grid resolutions, however, influence the synthetic disks noticeably. A look at the ratio of azimuthal to radial flux vector points at the importance of a well-sampled grid. While the setup I and II models show $F_{\varphi} / F_{r} \approx 0.08$ and 0.13 in the midplane, respectively, the same ratio for setup III with twice the number of polar solid angle points $n_{\theta}$ provides a ratio of $\sim 10^{-7}$ for $R<0.05$ AU. Furthermore, the ratio $F_{z} / F_{r}>1$ in setup III at radii $R \gtrsim 0.05 \mathrm{AU}$, implying that the vertical radiative transfer dominates the radial one as soon as the strong stellar irradiation field is blocked by the inward material.

Since these types of simulations for the inner disk of $\mathrm{T}$ Tauri stars have never been performed in 3D before, it is difficult to give a comparison to literature results. Nevertheless, Muzerolle et al. (2004) carried out similar calculations for more luminous typical Herbig Ae stars (Dullemond et al., 2001) using complete dust-free 1+1D models between $R=0.04-0.50$ AU and models with dust at larger radii. The dust inner radius of $R=0.50 \mathrm{AU}$ is the formal inner dust radius for a Herbig Ae star used for their calculations (see Eq. (1.4)); this radius for the $\mathrm{T}$ Tauri star used in the models presented here is $R \approx 0.017 \mathrm{AU}$, hence, within the inner radius of $R_{\text {in }}=0.030 \mathrm{AU}$ adopted in this model. For the lower accretion rate of $\dot{M}=10^{-9} M_{\odot} \mathrm{yr}^{-1}$, compared to $\dot{M}=3 \times 10^{-9} M_{\odot} \mathrm{yr}^{-1}$ used in the simulations carried our 
for this thesis, Muzerolle et al. (2004) find a complete optically thin gas disk while for the high accretion rate of $\dot{M}=10^{-6} M_{\odot} \mathrm{yr}^{-1}$ their model disk is still optically thin out to $R=0.25 \mathrm{AU}$. It is difficult to give a direct comparison between the results of Muzerolle et al. (2004) and the ones obtained here because of the differences in model parameters (e.g. Herbig Ae vs. T Tauri star). However, the models presented in this Section with a mass accretion rate of $\dot{M}=3 \times 10^{-9} M_{\odot} \mathrm{yr}^{-1}$ never become optically thin in the midplane assuming Rosseland mean opacities $\tau_{\text {ross }}$ in the determination of the $\tau>1$ region shown in Figs. 4.22 and 4.24. This is true even in the case where the complete midplane is hot enough to loose all its dust. Another difference between the two simulations is the inner disk radius. The models shown here have $R_{\mathrm{in}}=0.03 \mathrm{AU}$, hence, the very inner part is dense and blocks the stellar irradiation efficiently. This could be another reason for the different results.

Furthermore, in all three model setups the disk expands vertically by a factor of $\sim 2$. This blow-up is caused by the hydrostatic reaction of the disk material to the temperature increase. Only the setup I model, where dust is included as opacity source from the beginning on, shows a puffed-up inner disk rim which increases in height until $z_{\text {in }} \approx R_{\star}$. The other two models only display the overall vertical blow-up signatures. These different behaviours are probably a result of the different opacity binnings and of the fact that dust is included in setup I from the beginning on. The higher opacity in setup I leads to a quick increase in temperature $(T>3000 \mathrm{~K}$ at iteration 8 and after) in the upper layers at small radii which results in a strong hydrostatic blow-up. Once the disk is puffed-up and devoid of dust, the configuration seems to be hydrostatically stable and the temperature is kept at a high value of $\gtrsim 3000 \mathrm{~K}$ in the outer layers. The fast rise in temperature does not occur in setup II and III because dust is not considered as an opacity source in the first iterations. After dust is taken into account, the increase in temperature progresses rather slowly and maximum temperatures in the top layers of the very inner part of the disk stay below $2000 \mathrm{~K}$.

How the discussed properties of the disk models with direct irradiation depend on variations of the input parameters, e.g. mass accretion rate or inner disk radius, has not been analysed in this work due to lack of time. It could be shown that this method for modelling the structure of the inner disk taking into account direct irradiation of the central star is well suited and provides first interesting results. In the future, it would be helpful to accelerate the temperature convergence process, e.g. by $\mathrm{Ng}$ acceleration $(\mathrm{Ng}, 1974)$, and to increase the resolution of the model. 


\section{Chapter 5}

\section{Summary and outlook}

In the course of this thesis the well-tested stellar atmosphere program PHOENIX (Hauschildt \& Baron, 1999) has been extended in order to account for the physics of geometrically thin accretion disks. The structure equations, namely the hydrostatic equilibrium, the radiative transfer (including irradiation by the central star), as well as the radiative equilibrium, were adopted for the vertical structure of disks at given disk ring radii $R$. A set of these vertical structures for different distances of the disk annulus from the star can be combined in order to represent a $1+1 \mathrm{D}$ disk. A detailed description of the $1+1 \mathrm{D}$ disk model atmosphere calculations is given in Chapter 2. The accretion disk model adopted in this work basically applies to all disk-like structure that undergo accretion. It has shown that in the case of relatively cool protoplanetary disks, the model presented here works best for the very inner regions close to the star $(R \lesssim 1 \mathrm{AU})$ where temperatures are $>100 \mathrm{~K}$. These regions are warm enough for molecules to be abundant in the gas phase and hence a rich spectrum of gas lines is emitted. Due to the temperature inversion in the outer layers of the disk atmosphere produced by the stellar irradiation, the spectral lines are usually in emission. Due to the Keplerian rotation of the disk around the central star, the lines are broadened depending on the inclination of the disk with respect to the observer. The dependence of the emitted spectrum on the most important model parameters (stellar mass, stellar radius, and temperature, mass accretion rate, Reynolds number, etc.) was visualised and discussed in Sect. 2.11.

Disk model structures were then computed and compared to observed VLT CRIRES spectra of three classical T Tauri stars. The observations were focused on the CO fundamental transition region around $\sim 4.7 \mu \mathrm{m}$ which allows the simultaneous observation of many spectral lines, but does not show other strong species. In the case of GQ Lup, the inclination of the disk was determined to be $i \approx 22^{\circ}$, which is in the range of the value $i=27^{\circ} \pm 5^{\circ}$ measured by Broeg et al. (2007). A similar analysis as carried out by the latter authors was performed by Seperuelo Duarte et al. (2008) who also used spectro-photometric observations of the stellar activity cycle but received a significantly larger value for the inclination of $i=51^{\circ} \pm 13^{\circ}$. The near face-on disk of the well-studied CTTS TW Hya could be fitted with a disk model spectrum only if a high-energetic stellar emission source was added to the the blackbody spectrum of the star. Due to the small inclination of the system, the kinematic information of the disk is missing which made a clear constraint of the disk parameters difficult. The inner radius $R_{\text {in }}=0.09 \mathrm{AU}$ found in this analysis agrees fairly well with the results of previous works (Salvk et al., 2009; Eisner et al., 2006). The observed CO emission line spectrum of RNO 90 shows very broad line wings and flat-topped line centers, untypical for the classic accretion disk model. Hence, the synthetic line profiles do not fit the observations very well. A large improvement in the fit quality was achieved by assuming a 
turbulent velocity component in the disk as suggested by MHD simulations. Convolving the line profiles with a Gaussian of the width of the turbulent velocity produces flatter and broader line profiles which, however, still do not fit the line wings appropriately.

Overall the 1+1D disk structures, despite the simplifications of the accretion disk model and the 1D approach, can reproduce the observed spectra satisfyingly. In some cases modifications were necessary to improve the fit results. However, a detailed and quantitative analysis was hampered by the large amount of free parameters and the degeneracy of some. Therefore, a successful analysis demands high-quality observations, well-constrained stellar parameters (reduces the number of free parameters), and kinematic information from the observed system, i.e. a disk that is inclined with respect to the observer's view. Additionally, there are some issues on the simulation side that deserve consideration in the future. On the one hand, the current version of the DISK program calculates the slope of the disk surface with radius, an important parameter for the stellar irradiation treatment, only once at the beginning of a program. An iterative adaptation of this slope parameter will improve the the calculation of stellar heating and help to determine the temperature in the outer atmosphere layers, which is very important for the emission line strength, self-consistently. Furthermore, convective energy transport was not taken into account but will presumably also have an effect on the temperature structure and hence on the spectrum. One more obvious drawback of the DISK code is the assumption of equilibrium chemistry and local thermodynamic equilibrium. This is not very likely to be valid for the cool inner and optically thin and irradiated outer disk atmospheric layers. Therefore, the consideration of departure from chemical equilibrium due to turbulent mixing or photodissociation, and radiative excitation of molecules should be considered in future projects.

In this thesis the effort to model the inner regions of protoplanetary disks was extended to a full three dimensional treatment of the radiative transfer. Two particular problems have been investigated, namely the influence of the 3D RT on the line profile by means of a two-level atom line for symmetric and non-symmetric, i.e. disturbed, disks and the effect of direct stellar irradiation and radial RT consideration on the disk structure.

In the case of 3D line simulations in symmetric disks it was found that the line profiles do not deviate from the $1+1 \mathrm{D}$ lines for inclinations $i<40^{\circ}$, if in the latter case a sufficient number of disk rings is considered. In a next step, the $3 \mathrm{D}$ line profiles were calculated for non-axisymmetic disk structures. On the one side, a single density wave running through the disk was considered. This spiral arm was assumed to be adiabatic and the density was increased by a factor of 1.5. In this case a clear asymmetry in the line profile for non-zero inclinations was detectable and the shape of the line for different azimuthal viewing angles is shown. On the other side, hydrodynamic simulations of planets in disks by the Kley group (e.g. Klev \& Crida, 2008; Klev et al., 2009) - originally performed for Jupiter-type planets were scaled to the inner disk region (shrinking the Jupiter to a Mercury-size planet) - were considered in the 3D line calculations and again density fluctuations were assumed to be adiabatic. Calculations were performed for different orbital simulation-periods of the embedded planet (RHD models) and it was found that only for the planet which has just started to disturb the disk (i.e. the models with small number of orbital periods), the line profile is detectably altered. The general draw-back of the attempt to detect planets with this method is that also other than planet-related scenarios are potential triggers for disk inhomogeneities. In order to investigate the influence of embedded planets on the line profile more thoroughly in the future, realistic 3D hydrodynamic simulations for the inner disk regions are necessary.

The investigation of the influence of direct stellar irradiation onto the disk structure was split into two parts. A set of $1 \mathrm{D}$ structures was interpolated on a 3D grid and the monochromatic 
opacities were binned in order to save computation time in the 3D RT simulations. The new mean intensities were input to new temperature calculations for each grid point which were then used in the 1D simulations to solve the hydrostatic equilibrium equation and to update the opacities, this time for a large number of wavelength points. This procedure was iterated until convergence in temperature was achieved. The results show that the inner disk wall, which in the 3D case is directly irradiated opposite to the 1D case where only vertical radiative transfer and irradiation onto the disk surface is considered, is heated up significantly. This additional heating is mainly caused by the radial radiative transfer in the disk and not by the 3D treatment of the irradiation onto the upper layers of the disk atmosphere. In general, all simulated disk models show an overall temperature increase accompanied by a hydrostatic vertical blow-up. Large parts of the disk exceed the dust evaporation temperature of $1500 \mathrm{~K}$ but the remaining gaseous material does not become optically thin. A disk model with a higher resolution in spatial and solid angle grid points would help to simulate the effect more realistically. Furthermore, a variation of the model parameters, such as mass accretion rate or inner disk radius, should help to complete our picture of the structure of the very inner disk. Due to the alternating call of 1D and 3D RT programs, the queue waiting time would by far exceed the speed gains on large scale parallel computers. Therefore, faster single-user machines are necessary to investigate this problem closer in the future. 



\section{Bibliography}

ADAM, J. (1990) Line formation in accretion disks. A\& A, 240, 541-550.

Adams, F. C., Lada, C. J. \& Shu, F. H. (1987a) Spectral evolution of young stellar objects. ApJ, 312, 788-806.

Adams, F. C. \& Shu, F. H. (1986) Infrared spectra of rotating protostars. ApJ, 308, 836-853.

Adams, F. C., Shu, F. H. \& Lada, C. J. (1987b) The Disks of T Tauri Stars with Flat Infrared Spectra. In Bulletin of the American Astronomical Society, vol. 19 of Bulletin of the American Astronomical Society.

Alencar, S. H. P. \& Batalha, C. (2002) Variability of Southern T Tauri Stars. II. The Spectral Variability of the Classical T Tauri Star TW Hydrae. ApJ, 571, 378-393.

Allard, F., Hauschildt, P., Alexander, D., Tamanai, A. \& Schweitzer, A. (2001) The Limiting Effects of Dust in Brown Dwarf Model Atmospheres. ApJ, 556, 357-372.

Andre, P., Ward-Thompson, D. \& Barsony, M. (1993) Submillimeter continuum observations of Rho Ophiuchi A - The candidate protostar VLA 1623 and prestellar clumps. ApJ, 406, $122-141$.

Appenzeller, I. \& Mundt, R. (1989) T Tauri stars. A $\& A$ Rev., 1, 291-334.

Auer, L. (2003) Formal Solution: EXPLICIT Answers. In Stellar Atmosphere Modeling, edited by I. Hubeny, D. Mihalas \& K. Werner, vol. 288 of Astronomical Society of the Pacific Conference Series.

Balbus, S. A. \& Hawley, J. F. (1991) A powerful local shear instability in weakly magnetized disks. I - Linear analysis. II - Nonlinear evolution. ApJ, 376, 214-233.

Baron, E. \& Hauschildt, P. H. (2007) A 3D radiative transfer framework. II. Line transfer problems. A\&A A 468, 255-261.

Baron, E., Hauschildt, P. H. \& Chen, B. (2009) A 3D radiative transfer framework. V. Homologous flows. A\&A, 498, 987-992.

Barrado y Navascués, D. \& Martín, E. L. (2003) An Empirical Criterion to Classify T Tauri Stars and Substellar Analogs Using Low-Resolution Optical Spectroscopy. AJ, 126, 2997-3006.

Bary, J. S., Weintraub, D. A. \& Kastner, J. H. (2002) Detection of Molecular Hydrogen Orbiting a "Naked" T Tauri Star. ApJ, 576, L73-L76. 
Bary, J. S., Weintraub, D. A. \& Kastner, J. H. (2003) Detections of Rovibrational $\mathrm{H}_{2}$ Emission from the Disks of T Tauri Stars. ApJ, 586, 1136-1147.

Bath, G. T., Pringle, J. E. \& Whelan, J. A. J. (1980) Spectrophotometry of dwarf novae in the wavelength range 1250-7500 A. MNRAS, 190, 185-194.

Bell, K. R. \& Lin, D. N. C. (1994) Using FU Orionis outbursts to constrain self-regulated protostellar disk models. ApJ, 427, 987-1004.

BIK, A. \& THI, W. F. (2004) Evidence for an inner molecular disk around massive Young Stellar Objects. $A \& A, 427$, L13-L16.

Blake, G. A. \& Boogert, A. C. A. (2004) High-Resolution 4.7 Micron Keck/NIRSPEC Spectroscopy of the CO Emission from the Disks Surrounding Herbig Ae Stars. ApJ, 606, L73-L76.

Blum, R. D., Barbosa, C. L., Damineli, A., Conti, P. S. \& Ridgway, S. (2004) Accretion Signatures from Massive Young Stellar Objects. ApJ, 617, 1167-1176.

Bodenheimer, P. (1965) Studies in Stellar Evolution. II. Lithium Depletion during the Pre-Main Contraction. ApJ, 142, 451-+.

Brauer, F., Dullemond, C. P. \& Henning, T. (2008) Coagulation, fragmentation and radial motion of solid particles in protoplanetary disks. $A \mathscr{G} A, \mathbf{4 8 0}, 859-877$.

Broeg, C., Schmidt, T. O. B., Guenther, E., Gaedke, A., Bedalov, A., Neuhäuser, R. \& Walter, F. M. (2007) Rotational period of GQ Lupi. AESA, 468, 1039-1044.

Calvet, N., D’Alessio, P., Hartmann, L., Wilner, D., Walsh, A. \& Sitko, M. (2002) Evidence for a Developing Gap in a 10 Myr Old Protoplanetary Disk. ApJ, 568, 1008-1016.

Calvet, N. \& Gullbring, E. (1998) The Structure and Emission of the Accretion Shock in T Tauri Stars. ApJ, 509, 802-818.

Calvet, N., Patino, A., Magris, G. C. \& D'Alessio, P. (1991) Irradiation of accretion disks around young objects. I - Near-infrared CO bands. ApJ, 380, 617-630.

Cannizzo, J. K., Ghosh, P. \& Wheeler, J. C. (1982) Convective accretion disks and the onset of dwarf nova outbursts. ApJ, 260, L83-L86.

CARR, J. S. (1989) Near-infrared CO emission in young stellar objects. ApJ, 345, 522-535.

CARr, J. S. (2007) Gas at the inner disk edge. In IAU Symposium, edited by J. Bouvier \& I. Appenzeller, vol. 243 of IAU Symposium.

CARr, J. S. \& NaJita, J. R. (2008) Organic Molecules and Water in the Planet Formation Region of Young Circumstellar Disks. Science, 319, 1504-.

Carr, J. S., Tokunaga, A. T. \& Najita, J. (2004) Hot $\mathrm{H}_{2} \mathrm{O}$ Emission and Evidence for Turbulence in the Disk of a Young Star. ApJ, 603, 213-220.

Chandler, C. J., Carlstrom, J. E., Scoville, N. Z., Dent, W. R. F. \& Geballe, T. R. (1993) Infrared CO emission from young stars - High-resolution spectroscopy. ApJ, 412, L71-L74. 
Chiang, E. I. \& Goldreich, P. (1997) Spectral Energy Distributions of T Tauri Stars with Passive Circumstellar Disks. ApJ, 490, 368-+.

ClAret, A. (2004) A new non-linear limb-darkening law for LTE stellar atmosphere models III. Sloan filters: Calculations for $-5.0 \leq \log [\mathrm{M} / \mathrm{H}] \leq+1,2000 \mathrm{~K} \leq T_{\text {eff }} \leq 50000 \mathrm{~K}$ at several surface gravities. $A \mathscr{E} A, \mathbf{4 2 8}, 1001-1005$.

Cohen, M. \& Kuni, L. V. (1979) Observational studies of pre-main-sequence evolution. ApJS, 41, 743-843.

D’Alessio, P., Canto, J., Calvet, N. \& Lizano, S. (1998) Accretion Disks around Young Objects. I. The Detailed Vertical Structure. ApJ, 500, 411-+.

de Val-Borro, M., Edgar, R. G., Artymowicz, P., Ciecielag, P., Cresswell, P., D’Angelo, G., Delgado-Donate, E. J., Dirksen, G. et Al. (2006) A comparative study of disc-planet interaction. MNRAS, 370, 529-558.

Dreizler, S. (2003) Temperature Correction Schemes. In Stellar Atmosphere Modeling, edited by I. Hubeny, D. Mihalas \& K. Werner, vol. 288 of Astronomical Society of the Pacific Conference Series.

Dullemond, C. P. \& Dominik, C. (2004) Flaring vs. self-shadowed disks: The SEDs of Herbig Ae/Be stars. A\&A, 417, 159-168.

Dullemond, C. P., Dominik, C. \& Natta, A. (2001) Passive Irradiated Circumstellar Disks with an Inner Hole. ApJ, 560, 957-969.

Dullemond, C. P., Hollenbach, D., Kamp, I. \& D’Alessio, P. (2007) Models of the Structure and Evolution of Protoplanetary Disks. In Protostars and Planets V, edited by B. Reipurth, D. Jewitt \& K. Keil.

Dullemond, C. P. \& Turolla, R. (2000) An efficient algorithm for two-dimensional radiative transfer in axisymmetric circumstellar envelopes and disks. A $\mathscr{G} A, \mathbf{3 6 0}, 1187-1202$.

Dullemond, C. P., van Zadelhoff, G. J. \& Natta, A. (2002) Vertical structure models of T Tauri and Herbig Ae/Be disks. A\&A, 389, 464-474.

Eisner, J. A., Chiang, E. I. \& Hillenbrand, L. A. (2006) Spatially Resolving the Inner Disk of TW Hydrae. ApJ, 637, L133-L136.

Ferguson, J. W., Alexander, D. R., Allard, F., Barman, T., Bodnarik, J. G., Hauschildt, P. H., Heffner-Wong, A. \& Tamanai, A. (2005) Low-Temperature Opacities. ApJ, 623, 585-596.

FrICKE, K. (1969) Stability of Rotating Stars II. The Influence of Toroidal and Poloidal Magnetic Fields. $A \mathscr{G} A, \mathbf{1}, 388-+$.

Gammie, C. F. (1996) Layered Accretion in T Tauri Disks. ApJ, 457, 355-+.

Gardner, J. P., Mather, J. C., Clampin, M., Doyon, R., Greenhouse, M. A., Hammel, H. B., Hutchings, J. B., Jakobsen, P. et Al. (2006) The James Webb Space Telescope. Space Science Reviews, 123, 485-606. 
Gibb, E. L., Van Brunt, K. A., Brittain, S. D. \& Rettig, T. W. (2007) Warm HCN, $\mathrm{C}_{2} \mathrm{H}_{2}$, and $\mathrm{CO}$ in the Disk of GV Tau. ApJ, 660, 1572-1579.

Grevesse, N. \& Noels (1993) In Abundances, edited by C. Jaschek \& M. Jaschek, Kluwer, Dordrecht, p. 111.

Günther, H. M., Schmitt, J. H. M. M., Robrade, J. \& Liefke, C. (2007) X-ray emission from classical T Tauri stars: accretion shocks and coronae? A\& A, 466, 1111-1121.

Hamaguchi, K., Yamauchi, S. \& Koyama, K. (2005) X-Ray Study of Herbig Ae/Be Stars. ApJ, 618, 360-384.

Hauschildt, P. H. \& Baron, E. (1999) Numerical Solution of the Expanding Stellar Atmosphere Problem. Journal of Computational and Applied Mathematics, 109, 41-63.

Hauschildt, P. H. \& BARon, E. (2006) A 3D radiative transfer framework. I. Non-local operator splitting and continuum scattering problems. A\&A, 451, 273-284.

Hauschildt, P. H. \& Baron, E. (2008) A 3D radiative transfer framework. III. Periodic boundary conditions. $A \mathscr{E} A, 490,873-877$.

Hauschildt, P. H. \& BAron, E. (2009) A 3D radiative transfer framework. IV. Spherical and cylindrical coordinate systems. A\&A, 498, 981-985.

Hauschildt, P. H., Störzer, H. \& Baron, E. (1994) Convergence properties of the accelerated $\Lambda$-iteration method for the solution of radiative transfer problems. JQSRT, $51,875$.

Helling, C., Ackerman, A., Allard, F., Dehn, M., Hauschildt, P., Homeier, D., LodDers, K., MARley, M. ET AL. (2008) A comparison of chemistry and dust cloud formation in ultracool dwarf model atmospheres. MNRAS, 391, 1854-1873.

Herbig, G. H. \& Bell, K. R. (1988) Catalog of emission line stars of the orion population : 3 : 1988 .

Herczeg, G. J., Wood, B. E., Linsky, J. L., Valenti, J. A. \& Johns-Krull, C. M. (2004) The Far-Ultraviolet Spectra of TW Hydrae. II. Models of $\mathrm{H}_{2}$ Fluorescence in a Disk. ApJ, 607, 369-383.

Horne, K. \& MARsh, T. R. (1986) Emission line formation in accretion discs. MNRAS, 218, $761-773$.

Hubeny, I. (1990) Vertical structure of accretion disks - A simplified analytical model. ApJ, 351, $632-641$.

Huélamo, N., Figueira, P., Bonfils, X., Santos, N. C., Pepe, F., Gillon, M., Azevedo, R., Barman, T. ET AL. (2008) TW ;Hydrae: evidence of stellar spots instead of a Hot Jupiter. $A \mathscr{G} A$, 489, L9-L13.

Hueso, R. \& Guillot, T. (2005) Evolution of protoplanetary disks: constraints from DM Tauri and GM Aurigae. $A \& A, 442,703-725$.

Hügelmeyer, S. D., Dreizler, S., Hauschildt, P. H., Seifahrt, A., Homeier, D. \& BARMAn, T. (2009) Radiative transfer in circumstellar disks. I. 1D models for GQ Lupi. A\&SA, 498, 793-800. 
Janson, M., Brandner, W., Henning, T. \& Zinnecker, H. (2006) Early ComeOn+ adaptive optics observation of GQ Lupi and its substellar companion. A\&A, 453, 609-614.

Jayawardhana, R., Coffey, J., Scholz, A., Brandeker, A. \& van Kerkwijk, M. H. (2006) Accretion Disks around Young Stars: Lifetimes, Disk Locking, and Variability. ApJ, 648, 1206-1218.

Jayawardhana, R., Mohanty, S. \& Basri, G. (2003) Evidence for a T Tauri Phase in Young Brown Dwarfs. ApJ, 592, 282-287.

Johansen, A., Henning, T. \& Klahr, H. (2006) Dust Sedimentation and Self-sustained Kelvin-Helmholtz Turbulence in Protoplanetary Disk Midplanes. ApJ, 643, 1219-1232.

Johns-Krull, C. M. \& Valenti, J. A. (2001) New Infrared Veiling Measurements and Constraints on Accretion Disk Models for Classical T Tauri Stars. ApJ, 561, 1060-1073.

Kaeufl, H.-U., Ballester, P., Biereichel, P., Delabre, B., Donaldson, R., Dorn, R., Fedrigo, E., Finger, G. ET AL. (2004) CRIRES: a high-resolution infrared spectrograph for ESO's VLT. In Society of Photo-Optical Instrumentation Engineers (SPIE) Conference Series, edited by A. F. M. Moorwood \& M. Iye, vol. 5492 of Society of Photo-Optical Instrumentation Engineers (SPIE) Conference Series.

Kamp, I. \& Dullemond, C. P. (2004) The Gas Temperature in the Surface Layers of Protoplanetary Disks. ApJ, 615, 991-999.

Karttunen, H., Kroeger, P., Oja, H., Poutanen, M. \& Donner, K. J. (2003) Fundamental astronomy.

Kenyon, S. J. \& Hartmann, L. (1987) Spectral energy distributions of T Tauri stars - Disk flaring and limits on accretion. ApJ, 323, 714-733.

Kenyon, S. J. \& Hartmann, L. (1995) Pre-Main-Sequence Evolution in the Taurus-Auriga Molecular Cloud. ApJS, 101, 117-+.

Kiplinger, A. L. (1979) SS Cygni - The accretion disk in eruption and at minimum light. ApJ, 234, 997-1015.

KLAHr, H. (2007) Thermal convection in accretion disks. In IAU Symposium, edited by F. Kupka, I. Roxburgh \& K. Chan, vol. 239 of IAU Symposium.

Klahr, H. H. \& Bodenheimer, P. (2003) Turbulence in Accretion Disks: Vorticity Generation and Angular Momentum Transport via the Global Baroclinic Instability. ApJ, 582, 869-892.

Kley, W., Bitsch, B. \& Klahr, H. (2009) Planet migration in three-dimensional radiative discs. ArXiv e-prints.

Kley, W. \& CRIDA, A. (2008) Migration of protoplanets in radiative discs. AEA, 487, L9-L12.

Kriz, S. \& Hubeny, I. (1986) Models and theoretical spectra of accretion discs in dwarf novae. Bulletin of the Astronomical Institutes of Czechoslovakia, 37, 129-142.

Lacy, J. H., Knacke, R., Geballe, T. R. \& Tokunaga, A. T. (1994) Detection of absorption by $\mathrm{H} 2$ in molecular clouds: A direct measurement of the $\mathrm{H} 2$ : $\mathrm{CO}$ ratio. ApJ, 428, L69-L72. 
LAdA, C. J. (1987) Star formation - From OB associations to protostars. In Star Forming Regions, edited by M. Peimbert \& J. Jugaku, vol. 115 of IAU Symposium.

LadA, C. J. \& Wilking, B. A. (1984) The nature of the embedded population in the Rho Ophiuchi dark cloud - Mid-infrared observations. ApJ, 287, 610-621.

Lahuis, F., van Dishoeck, E. F., Blake, G. A., Evans, II, N. J., Kessler-Silacci, J. E. \& Pontoppidan, K. M. (2007) c2d Spitzer IRS Spectra of Disks around T Tauri Stars. III. [Ne II], [Fe I], and H_2 Gas-Phase Lines. ApJ, 665, 492-511.

Lahuis, F., van Dishoeck, E. F., Boogert, A. C. A., Pontoppidan, K. M., Blake, G. A., Dullemond, C. P., Evans, II, N. J., Hogerheijde, M. R. Et Al. (2006) Hot Organic Molecules toward a Young Low-Mass Star: A Look at Inner Disk Chemistry. ApJ, 636, L145-L148.

Lucy, L. B. (1964) A Temperature-Correction Procedure. SAO Special Report, 167, 93-+.

Ludwig, H.-G. (1992) PhD thesis.

Lynden-Bell, D. (1969) Galactic Nuclei as Collapsed Old Quasars. Nature, 223, 690-694.

Lynden-Bell, D. \& Pringle, J. E. (1974) The evolution of viscous discs and the origin of the nebular variables. MNRAS, 168, 603-637.

Mayo, S. K., Wickramasinghe, D. T. \& Whelan, J. A. J. (1980) Theory and observations of the optical continuum and line spectra of accretion discs around white dwarfs. MNRAS, 193, 793-824.

Meyer, F. \& Meyer-Hofmeister, E. (1982) Vertical structure of accretion disks. A\&A, 106, $34-42$.

Minalas, D. (1978) Stellar atmospheres /2nd edition/.

Muzerolle, J., Calvet, N., Briceño, C., Hartmann, L. \& Hillenbrand, L. (2000) Disk Accretion in the 10 MYR Old T Tauri Stars TW Hydrae and Hen 3-600A. ApJ, 535, L47-L50.

Muzerolle, J., D’Alessio, P., Calvet, N. \& Hartmann, L. (2004) Magnetospheres and Disk Accretion in Herbig Ae/Be Stars. ApJ, 617, 406-417.

Nagel, T., Dreizler, S., Rauch, T. \& Werner, K. (2004) AcDc - A new code for the NLTE spectral analysis of accretion discs: application to the helium CV AM CVn. $A \& A, 428$, $109-115$.

Najita, J., Carr, J. S., Glassgold, A. E., Shu, F. H. \& Tokunaga, A. T. (1996) Kinematic Diagnostics of Disks around Young Stars: CO Overtone Emission from WL 16 and 1548C27. ApJ, 462, 919-+.

Najita, J., Carr, J. S. \& Mathieu, R. D. (2003) Gas in the Terrestrial Planet Region of Disks: CO Fundamental Emission from T Tauri Stars. ApJ, 589, 931-952.

Najita, J. R., Carr, J. S., Glassgold, A. E. \& Valenti, J. A. (2007) Gaseous Inner Disks. In Protostars and Planets V, edited by B. Reipurth, D. Jewitt \& K. Keil. 
Natta, A., Prusti, T., Neri, R., Wooden, D., Grinin, V. P. \& Mannings, V. (2001) A reconsideration of disk properties in Herbig Ae stars. A\&A, 371, 186-197.

Natta, A., Testi, L., Calvet, N., Henning, T., Waters, R. \& Wilner, D. (2007) Dust in Protoplanetary Disks: Properties and Evolution. In Protostars and Planets $V$, edited by B. Reipurth, D. Jewitt \& K. Keil.

Natta, A., Testi, L., Muzerolle, J., Randich, S., Comerón, F. \& Persi, P. (2004) Accretion in brown dwarfs: An infrared view. A\&H, 424, 603-612.

Neuhäuser, R., Guenther, E. W., Wuchterl, G., Mugrauer, M., Bedalov, A. \& Hauschildt, P. H. (2005) Evidence for a co-moving sub-stellar companion of GQ Lup. $A \& A$, 435, L13-L16.

NG, K. (1974) Hypernetted chain solutions for the classical one-component plasma up to Gamma equals 7000. J. Chem. Phys., 61, 2680-2689.

Norduund, A. (1982) Numerical simulations of the solar granulation. I - Basic equations and methods. $A \mathscr{E} A, 107,1-10$.

Olson, G. L. \& Kunasz, P. B. (1987) Short characteristic solution of the non-LTE transfer problem by operator perturbation. I. The one-dimensional planar slab. Journal of Quantitative Spectroscopy and Radiative Transfer, 38, 325-336.

Pascucci, I., Wolf, S., Steinacker, J., Dullemond, C. P., Henning, T., Niccolini, G., Woitke, P. \& Lopez, B. (2004) The 2D continuum radiative transfer problem. Benchmark results for disk configurations. $A \mathscr{E} A, 4 \mathbf{4 1 7}, 793-805$.

Perez, M. R. \& Grady, C. A. (1997) Observational Overview of Young Intermediate-Mass Objects: Herbig Ae/Be Stars. Space Science Reviews, 82, 407-450.

Pollack, J. B., Hollenbach, D., Beckwith, S., Simonelli, D. P., Roush, T. \& Fong, W. (1994) Composition and radiative properties of grains in molecular clouds and accretion disks. ApJ, 421, 615-639.

Pontoppidan, K. M., Blake, G. A., van Dishoeck, E. F., Smette, A., Ireland, M. J. \& Brown, J. (2008) Spectroastrometric Imaging of Molecular Gas within Protoplanetary Disk Gaps. ApJ, 684, 1323-1329.

Rettig, T. W., Haywood, J., Simon, T., Brittain, S. D. \& Gibb, E. (2004) Discovery of CO Gas in the Inner Disk of TW Hydrae. ApJ, 616, L163-L166.

Salyk, C., Blake, G. A., Boogert, A. C. A. \& Brown, J. M. (2007) Molecular Gas in the Inner 1 AU of the TW Hya and GM Aur Transitional Disks. ApJ, 655, L105-L108.

Salyk, C., Blake, G. A., Boogert, A. C. A. \& Brown, J. M. (2009) High-resolution $5 \mu$ m Spectroscopy of Transitional Disks. ApJ, 699, 330-347.

Scoville, N., Kleinmann, S. G., Hall, D. N. B. \& Ridgway, S. T. (1983) The circumstellar and nebular environment of the Becklin-Neugebauer object - 2-5 micron wavelength spectroscopy. ApJ, 275, 201-224. 
Seperuelo Duarte, E., Alencar, S. H. P., Batalha, C. \& Lopes, D. (2008) Spectrophotometric analysis of the T Tauri star GQ Lupi A. A\&A, 489, 349-357.

Setiaman, J., Henning, T., Launhardt, R., Müller, A., Weise, P. \& Kürster, M. (2008) A young massive planet in a star-disk system. Nature, 451, 38-41.

Shakura, N. I. \& Sunyaev, R. A. (1973) Black holes in binary systems. Observational appearance. $A \& A, \mathbf{2 4}, 337-355$.

Shaviv, G. \& Wehrse, R. (1986) The vertical temperature stratification and corona formation of accretion disc atmospheres. $A \mathscr{E} A, \mathbf{1 5 9}, \mathrm{L} 5-\mathrm{L} 7$.

Shu, F., Najita, J., Ostriker, E., Wilkin, F., Ruden, S. \& Lizano, S. (1994) Magnetocentrifugally driven flows from young stars and disks. 1: A generalized model. ApJ, 429, $781-796$.

Siess, L., Dufour, E. \& Forestini, M. (2000) An internet server for pre-main sequence tracks of low- and intermediate-mass stars. A\&A, 358, 593-599.

Sitko, M. L., Lynch, D. K. \& Russell, R. W. (2000) Silicate Emission in the TW Hydrae Association. AJ, 120, 2609-2614.

Smith, R. L., Pontoppidan, K. M., Young, E. D., Morris, M. R. \& van Dishoeck, E. F. (2009) High-Precision $\mathrm{C}^{17} \mathrm{O}, \mathrm{C}^{18} \mathrm{O}$, and $\mathrm{C}^{16} \mathrm{O}$ Measurements in Young Stellar Objects: Analogues for CO Self-shielding in the Early Solar System. ApJ, 701, 163-175.

Steinacker, J., Henning, T., Bacmann, A. \& Semenov, D. (2003) 3D continuum radiative transfer in complex dust configurations around stellar objects and active galactic nuclei. I. Computational methods and capabilities. A\&̈A, 401, 405-418.

Strom, S. E. \& Kurucz, R. (1966) Statistical Procedure for Computing Line-Blanketed Model Stellar Atmospheres. AJ, 71, 181-+.

ThI, W.-F. \& BIK, A. (2005) Detection of steam in the circumstellar disk around a massive Young Stellar Object. $A \& A, 438,557-570$.

Thi, W.-F., van Zadelhoff, G.-J. \& van Dishoeck, E. F. (2004) Organic molecules in protoplanetary disks around T Tauri and Herbig Ae stars. A\&SA, 425, 955-972.

Tielens, A. G. G. M. \& Hollenbach, D. (1985) Photodissociation regions. I - Basic model. II - A model for the Orion photodissociation region. ApJ, 291, 722-754.

Vögler, A., Bruls, J. H. M. J. \& Schüssler, M. (2004) Approximations for non-grey radiative transfer in numerical simulations of the solar photosphere. $A \& A, \mathbf{4 2 1}, 741-754$.

WADE, R. A. (1988) A test of synthetic accretion disk spectra using ultraviolet flux distributions of novalike variables. ApJ, 335, 394-405.

Waters, L. B. F. M. \& Waelkens, C. (1998) Herbig Ae/Be Stars. ARA\& A, 36, 233-266.

Weinberger, A. J., Becklin, E. E., Schneider, G., Chiang, E. I., Lowrance, P. J., Silverstone, M., Zuckerman, B., Hines, D. C. ET Al. (2002) Infrared Views of the TW Hydra Disk. ApJ, 566, 409-418. 
Weintraub, D. A., Kastner, J. H. \& Bary, J. S. (2000) Detection of Quiescent Molecular Hydrogen Gas in the Circumstellar Disk of a Classical T Tauri Star. ApJ, 541, 767-771.

Weintraub, D. A., Tegler, S. C., Kastner, J. H. \& Rettig, T. (1994) Infrared Spectroscopy and Imaging Polarimetry of the Disk around the T Tauri Star RNO 91. ApJ, 423, $674-+$.

Wilner, D. J., D’Alessio, P., Calvet, N., Claussen, M. J. \& Hartmann, L. (2005) Toward Planetesimals in the Disk around TW Hydrae: 3.5 Centimeter Dust Emission. ApJ, 626, L109-L112.

Woitke, P., Kamp, I. \& Thi, W. (2009) Radiation thermo-chemical models of protoplanetary disks. I. Hydrostatic disk structure and inner rim. A\&GA, 501, 383-406.

Wolf, S., Henning, T. \& Stecklum, B. (1999) Multidimensional self-consistent radiative transfer simulations based on the Monte-Carlo method. A\& A, 349, 839-850.

Woods, P. M. \& Willacy, K. (2009) Carbon Isotope Fractionation in Protoplanetary Disks. ApJ, 693, 1360-1378.

Yamashita, T., Handa, T., Omodaka, T., Kitamura, Y., Kawazoe, E., Hayashi, S. S. \& KAIfU, N. (1993) Upper limits to the CO J = 1-0 emission around Vega-like stars - Gas depletion of the circumstellar ring around Epsilon Eridani. ApJ, 402, L65-L67. 



\section{Appendix A}

\section{D line profile contributions}

This chapter provides plots of line profiles for the different 3D disk setups presented in Sect. 4.3. The total disk-integrated line profiles, which are also displayed in Sect. 4.3, are shown together with contributions from five annuli. With this approach the origin of the disk-integrated line variation due to disk asymmetries can be broken down into different disk regions and a comprehensive understanding is possible. 


\section{A.1 Density wave}
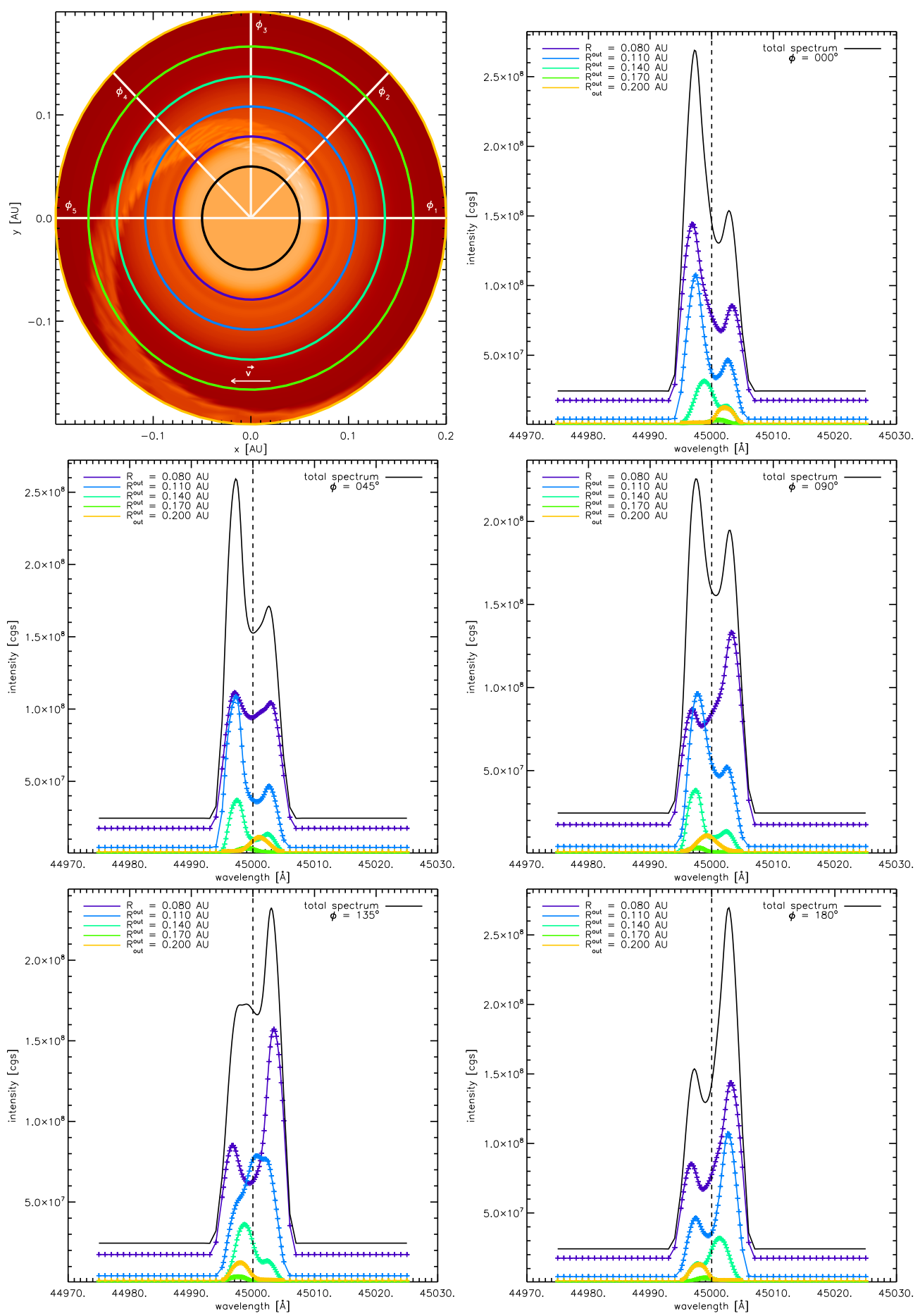

Figure A.1: Line contributions from different regions of the disk with density wave for azimuthal viewing angles $\phi=0^{\circ}, 45^{\circ}, 90^{\circ}, 135^{\circ}$, and $180^{\circ}$ (left to right, top to bottom) and constant inclination $i=20^{\circ}$. The opacity contour plot (top left) marks the annuli and viewing angles $\phi$ for which single line profiles are given. 


\section{A.2 Disk-planet model at $t=5$ orbits}
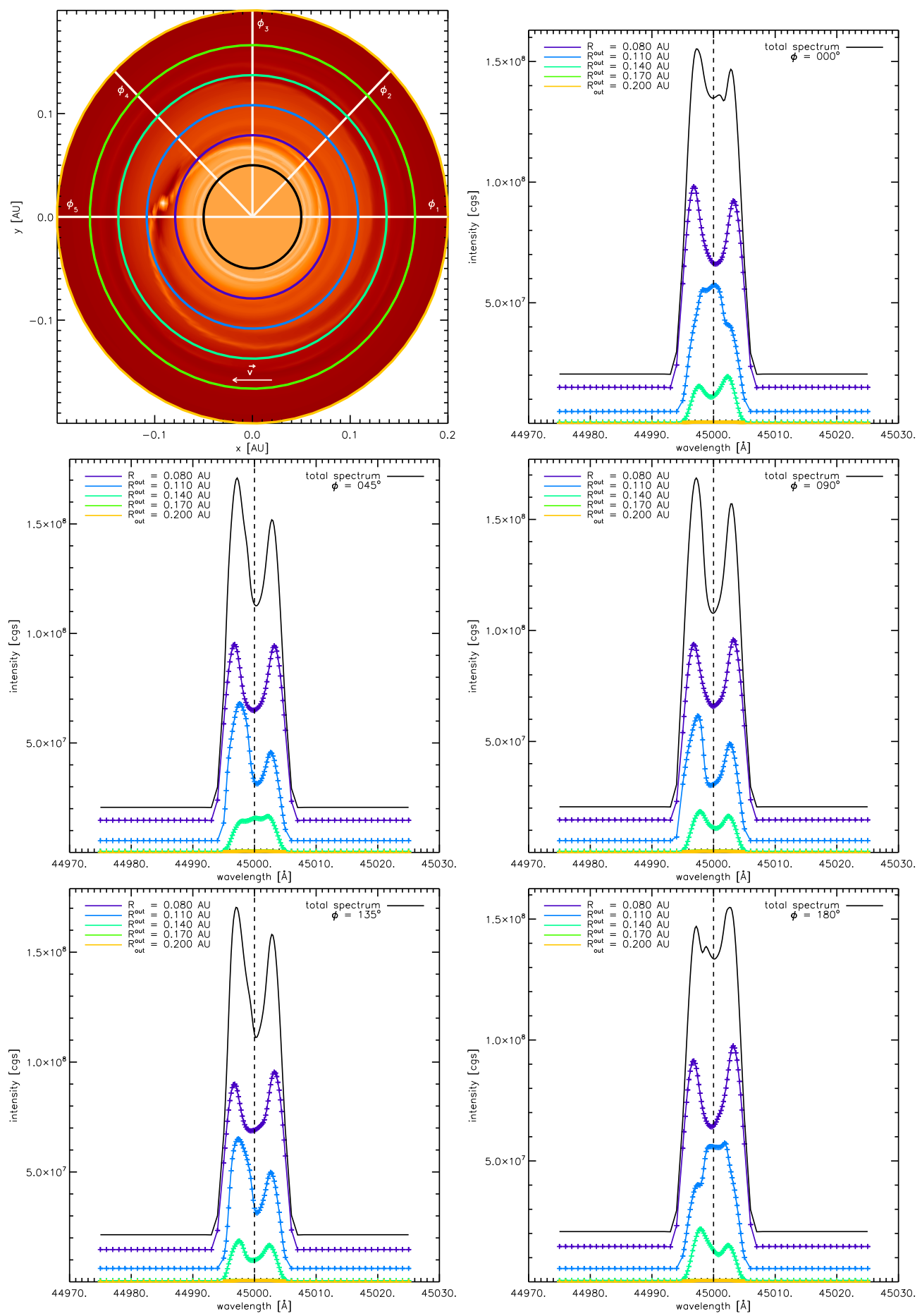

Figure A.2: Line contributions from different regions of the disk for the $t=5$ orbits disk-planet setup and viewing angles $\phi=0^{\circ}, 45^{\circ}, 90^{\circ}, 135^{\circ}$, and $180^{\circ}$ (left to right, top to bottom) and constant inclination $i=20^{\circ}$. The opacity contour plot (top left) marks the annuli and viewing angles $\phi$ for which single line profiles are given. 


\section{A.3 Disk-planet model at $t=50$ orbits}
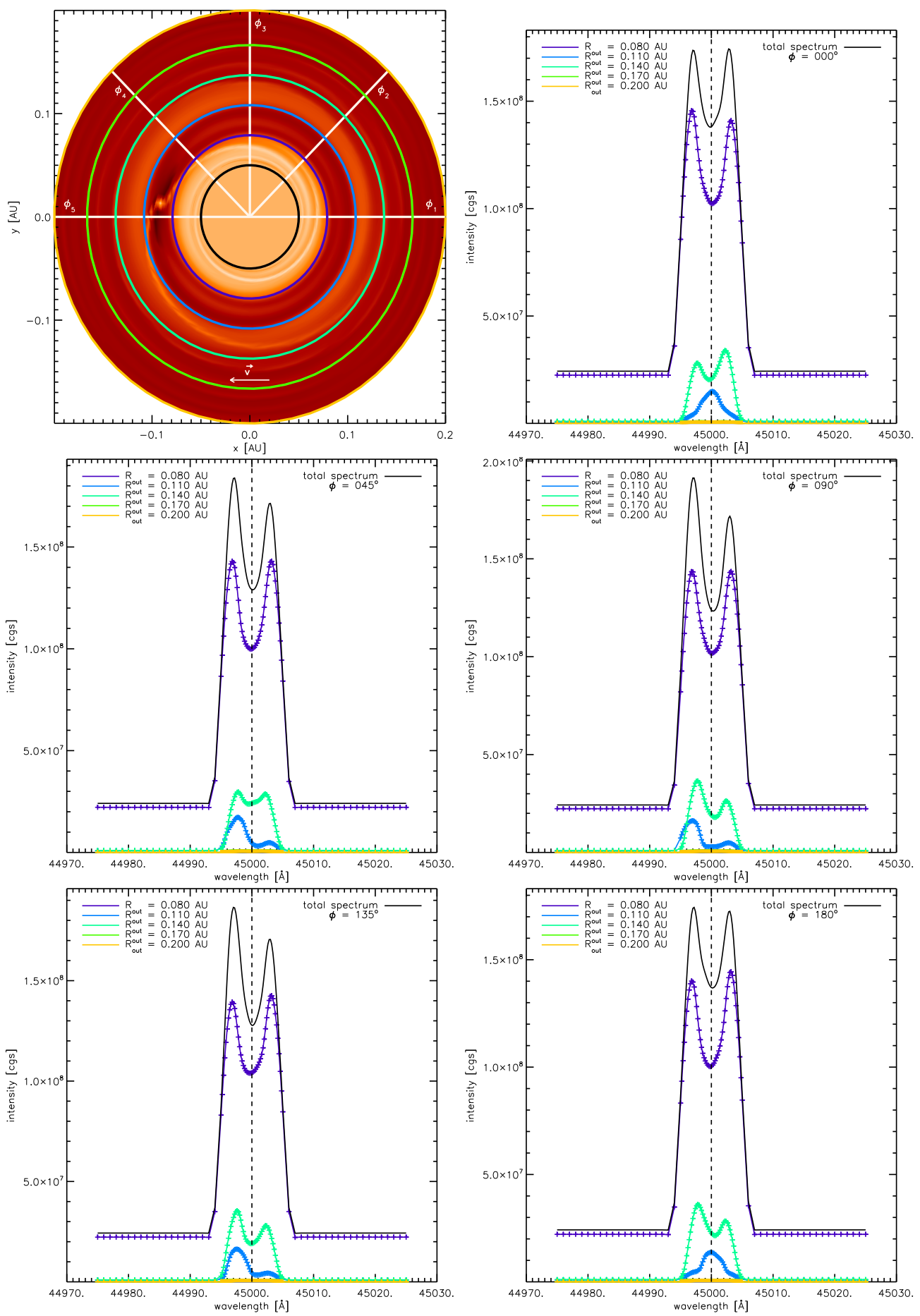

Figure A.3: Line contributions from different regions of the disk for the $t=50$ orbits disk-planet setup and viewing angles $\phi=0^{\circ}, 45^{\circ}, 90^{\circ}, 135^{\circ}$, and $180^{\circ}$ (left to right, top to bottom) and constant inclination $i=20^{\circ}$. The opacity contour plot (top left) marks the annuli and viewing angles $\phi$ for which single line profiles are given. 


\section{Appendix B}

\section{PHOENIX DISK mode}

This chapter is thought to be an extension to the PHOENIX manual 1 for the DISK mode. All necessary steps to run a $1 \mathrm{D}$ disk ring model job will be described.

\section{B.1 DISK input parameters}

- model: For all disk models, model $=9$.

- job: A new start structure is calculated for $\mathrm{job}=0$. If an existing input model is supposed to be read in, one needs to set $\mathrm{job}=10$.

- mstar: Mass of central star in solar masses (default mstar=1.d0).

- rstar: Radius of central star in solar radii (default rstar=1.d0).

- mdotr: Mass accretion rate in solar masses per year (default mdotr=1.d-8).

- reynr: Reynolds number as a measure for the viscosity (default reynr=1.d4).

- rdiskr: Radius of the disk ring in AU (default rdiskr=1.d0).

- dampfac: Damping factor for the viscosity; should be set to a value smaller than 1 if numerical problems in the outer layers occur (default dampfac=1.d0).

- viscdep: Factor describing the depth-dependency of the viscosity; for constant viscosity set equal to 0.d0, for total depth-dependent viscosity set equal to 1.d0; if e.g. inner $90 \%$ of column mass has constant viscosity set to $0.1 \mathrm{~d} 0$ (default viscdep=1.d0).

- dul_pd: Logical variable if set true activates "passive damping" in the Unsöld-Lucy temperature correction scheme (default dul_pd=.false.).

- pd_shift: Determines damping factors: d1=exp(-tau/pd_shift) and d2=1.d0-d1 (default pd_shift=1.d0).

- rhcor_min: Termination criterion for structure iteration; if relative flux change is smaller than rhcor_min in all layers than finish (default rhcor_min=1.d-2).

- $1 \mathrm{~cm} \_m i n:$ Minimum logarithmic column mass density of disk atmosphere; should be set to smaller value if lines are still optically thick in top layer (default $1 \mathrm{~cm} \_m i n=-4$ ).

- ninpts: Number of points set very close to midplane; a large value increases numerical stability in these layers (default ninpts $=6$ ).

- twd: Temperature of central star $[\mathrm{K}]$ (default twd=0).

- Iwd: Luminosity of central star [cgs] (default $1 w d=0)$.

- d2: Distance between central star and disk ring $[\mathrm{cm}]$ (default d2=0).

- mkhint: Solves RT twice - with and without irradiation (default mkhint=false).

${ }^{1}$ http://www.hs.uni-hamburg.de/EN/For/ThA/phoenix/index.html 
- slope_factor_disk: Fudge factor for slope of disk height with radius (default slope_factor_disk=1.5).

- tuv: Temperature of stellar UV radiation (default tuv $=0$ ).

- tuv: Surface fraction of stellar UV radiation (default fuv=0).

- max_spectrum_points: Maximum number of points for an irradiation stellar model (PHOENIX intensity spectrum). The variable determines whether or not a model is read in (max_spectrum_points $>0$ ) or if a blackbody is used as irradiation source (default max_spectrum_points $=-1$ ).

\section{B.2 DISK input files}

- disk_opac.tbl: Opacity table 2 of Ferguson et al. (2005); the entry "log T" needs to be replaced by "0.000" !

- Id.dat: Table of limb darkening coefficients (Claret, 2004)

- fort.94: File that holds the angle dependent intensity spectrum (see lay_rf and irfout in the PHOENIX manua 3). This file is only needed if irradiation by a stellar model spectrum instead of a blackbody spectrum is desired. 


\title{
Acknowledgements / Danksagungen
}

\author{
Mein vorrangiger Dank gebührt meinem Doktorvater Stefan Dreizler, der diese Arbeit betreut \\ hat. Er hat mit mir in dieser Zeit, und bereits zuvor während meiner Diplomarbeit, mit großem \\ Enthusiasmus zusammengearbeitet und meine wissenschaftliche Entwicklung in höchstem Maße \\ gefördert. Sein Vertrauen in mich habe ich immer sehr zu schätzen gewusst. Für die vielen \\ spannenden Diskussionen zu Themen der Astrophysik, aber auch darüber hinaus, möchte ich \\ mich ganz herzlich bedanken. Ein persönlicheres und besseres Verhältnis zu seinem „Chef“ kann \\ ich mir nur schwer vorstellen.
}

Auch meinem Betreuer und Koreferenten dieser Arbeit Peter Hauschildt sei an dieser Stelle herzlichst für die sehr gute und entspannte Zusammenarbeit gedankt. In zahlreichen Treffen in der Hamburger Sternwarte und anderswo habe ich viel von ihm über die Tücken der numerischen Astrophysik lernen dürfen. Seine schnelle Reaktionszeit auf Anfragen jeder Art hat einen wesentlichen Teil zum Gelingen dieser Arbeit beigetragen.

Besonders danke ich ebenfalls Kees Dullemond, der mich großzügiger Weise nach Heidelberg eingeladen hat und von dem ich dort einen neuen Einblick in die Aspekte der Modellierung von Staubscheiben bekommen habe, und Willy Kley, ohne dessen Modelle und Tipps die Strahlungstransportsimulationen in Scheiben mit Planeten nicht möglich gewesen wäre.

Desweiteren möchte ich einigen großartigen Menschen danken, die mich in meiner Zeit am IAG begleitet haben: Thorsten Stahn hatte trotz seiner Arbeitgeber bedingten räumlichen Abwesenheit vom IAG dank moderner Kommunikationswege immer ein „offenes Ohr" für meinen gelegentlichen Unmut. Die gemeinsamen Abende mit Fußballvergnügen jeglicher Art bleiben unvergessen... Thooooooooooooooorsten!!! Andreas Seifahrt hat sowohl bei der Datenreduktion der CRIRES Spektren als auch als Quelle astrophysikalischer Ideen wertvolle Hilfe geleistet. Die gemeinsamen Kaffeerunden waren persönlich wie wissenschaftlich ein großer Gewinn für mich. Desweiteren möchte ich an dieser Stelle Derek Homeier als auch Ansgar Reiners danken. Beide haben mich duch wichtige Ratschläge in meiner Arbeit wesentlich unterstützt, Derek vor allem als lokaler Ansprechpartner zu Fragen bezüglich PHOENIX. Auch Sebastian Wende möchte ich ganz herzlich Danken für seine Langatmigkeit beim Diskutieren über phsyikalische Probleme. Die vielen Dienstreisen die wir gemeinsam machen durften bleiben mir als Höhepukte meiner Zeit am IAG in Erinnerung. Ronny Lutz möchte ich für die sportlichen Ablenkungen von der mitunter stressigen Arbeit danken. In dem Zusammenhang sei auch Nandan Joshi erwähnt, der die mit den sportlichen Aktivitäten verbundene Lärmbelästigung so tapfer ertragen hat. Vielen Dank für die nette Zeit in F04.139!

Außerdem sei an dieser Stelle den vielen wunderbaren Arbeitskollegen aus dem IAG gedankt, insbesondere Jacob Bean, Nicole Böcker, José Fernandez, Wolfgang Glatzel, Rick Hessman, Markus Hundertmark, Melanie Hüttenmeister, Wolfram Kollatschny, Maren Mohler, Klaus Reinsch, Sonja Schuh, Ulf Seemann, Denis Shulyak, David Swoboda, Iris Traulsen, Klaudia Wolters als auch dem 
Jungen und dem Mädchen. Meinen „alten“ Freunden aus Osnabrück, Stefan Müller-Nedebock, Henning und Jessica Rahne, Stefan Berendes, Malte-Boris Schrader, Mitja Stadje, sowie den „neuen“ aus Göttingen, Daniel Broxtermann, Britta Kreilein, Thomas Rademacher und Jakob Walowski möchte ich für eine schöne und abwechselungsreiche Studienzeit danken.

$\mathrm{Zu}$ guter Letzt danke ich meinen Eltern, Klaus und Renate Hügelmeyer, als auch meinem Bruder Philipp und meiner Freundin Karen Hollenberg für die liebevolle Unterstützung in allen Belangen. Danke dass ihr immer für mich da seid! Ohne euch wäre ich heute sicher nicht da wo ich bin. 


\section{Curriculum Vitae}

Dipl.-Phys. Simon Daniel Hügelmeyer

Theaterplatz 8

37073 Göttingen

Geburtstag:

16.05 .1980

Geburtsort:

Osnabrück

Staatsangehörigkeit:

deutsch

01/2007 - 12/2009 Promotionsstipendiat (Universität Göttingen)

07/2006 - 12/2006 Wissenschaftlicher Mitarbeiter (Universität Göttingen)

11.05.2006 Diplom Physik (Universität Göttingen)

10/2003 - 05/2006 Physikstudium (Universität Göttingen)

18.07.2003 Vordiplom Physik (Universität Osnabrück)

10/2001 - 09/2003 Physikstudium (Universität Osnabrück)

07/2000 - 05/2001 Zivildienst in Osnabrück

08.07.2000 allgemeine Hochschulreife

08/1997 - 07/2000 Ratsgymnasium Osnabrück

09/1997 - 07/1998 Concordia High School, Concordia, Kansas, USA

08/1993 - 08/1997 Ratsgymnasium Osnabrück

08/1991 - 07/1993 Orientierungsstufe „In der Wüste“ Osnabrück

08/1987 - 07/1991 Grundschule Rückertschule Osnabrück 